UNIVERSIDADE DE SÃO PAULO

ESCOLA DE ENGENHARIA DE SÃO CARLOS

RAISSA TAVARES VIEIRA

Descritores robustos à rotação de texturas baseados na abordagem LMP com acréscimo da informação de Magnitude e Sinal 



\section{Descritores robustos à rotação de texturas baseados na abordagem LMP com acréscimo da informação de Magnitude e Sinal}

Tese apresentada à Escola e Engenharia de São Carlos da Universidade de São Paulo para a obtenção do título de Doutora em Ciências, Programa de Engenharia Elétrica.

Área de concentração: Processamento de Sinais e Instrumentação

Orientador: Prof. Dr. Adilson Gonzaga

Trata-se da versão corrigida da tese. A versão originalse encontra disponível na EESC/USP que aloja o Programa de Pós-Graduação de Engenharia ELétrica

São Carlos 
AUTORIZO A REPRODUÇÃO TOTAL OU PARCIAL DESTE TRABALHO, POR QUALQUER MEIO CONVENCIONAL OU ELETRÔNICO, PARA FINS DE ESTUDO E PESQUISA, DESDE QUE CITADA A FONTE.

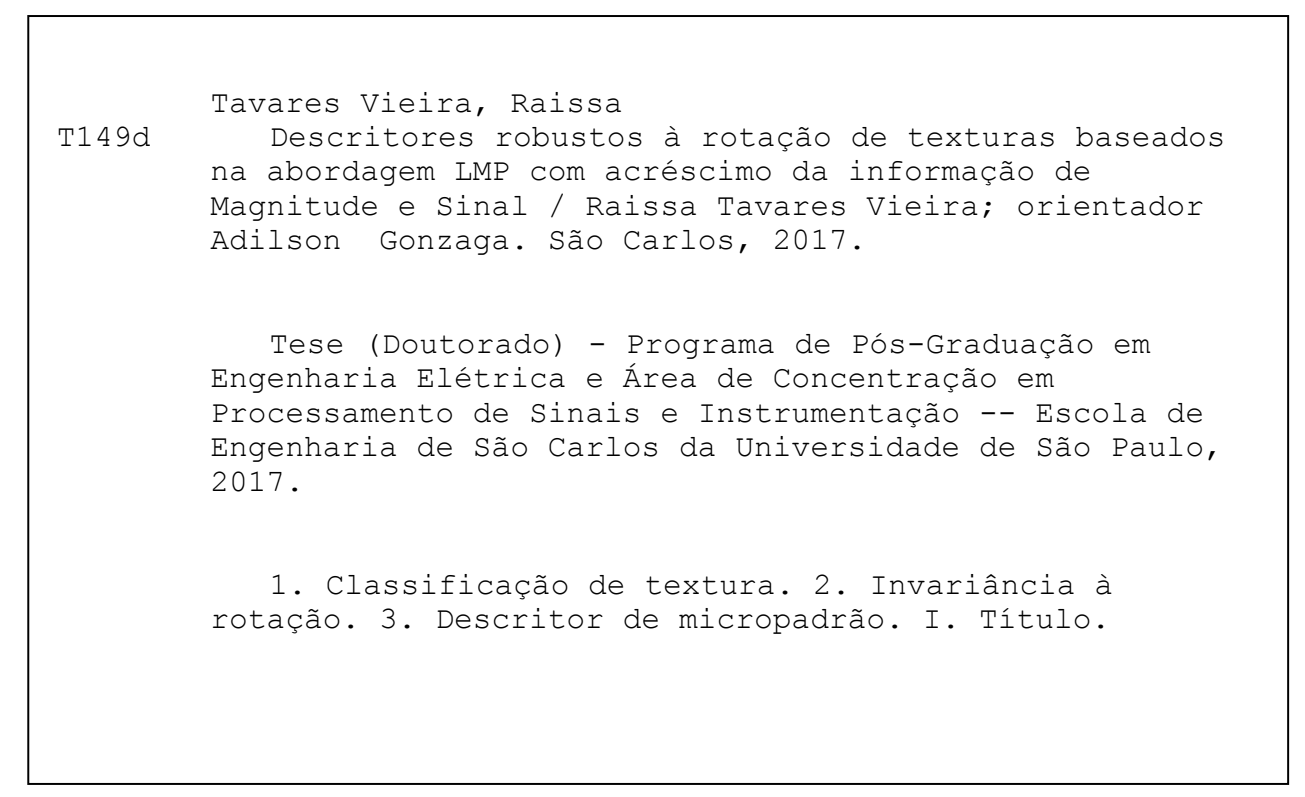


Candidata: RAISSA TAVARES VIEIRA.

Título da tese: "Descritores robustos à rotação de texturas baseados na abordagem LMP com acréscimo da informação de Magnitude e Sinal".

Data da defesa: 06/09/2017.

Comissão Julgadora:

Resultado:

Prof. Associado Adilson Gonzaga (Orientador)

APROVADA

(Escola de Engenharia de São Carlos/EESC)

Prof. Associado Evandro Luis Linhari Rodrigues

(Escola de Engenharia de São Carlos/EESC)

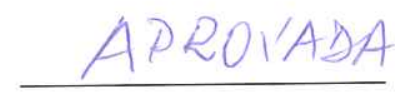

Prof. Dr. Marcelo Ricardo Stemmer

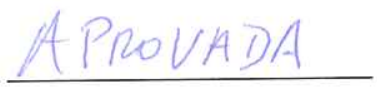

(Universidade Federal de Santa Catarina/UFSC)

Prof. Dr. Maurilio Boaventura

APRUVADA

(Universidade Estadual "Júlio de Mesquita Filho"/UNESP-Rio Claro)

Prof. Dr. Bruno Augusto Nassif Travençolo

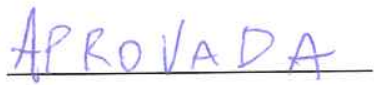

(Universidade Federal de Uberlândia/UFU)

Coordenador do Programa de Pós-Graduação em Engenharia Elétrica:

Prof. Associado Luís Fernando Costa Alberto

Presidente da Comissão de Pós-Graduação:

Prof. Associado Luís Fernando Costa Alberło 

Dedico este trabalho aos meus maiores incentivadores, Painho e Mainha. 



\section{AGRADECIMENTOS}

Agradeço ao meu orientador Prof. Dr. Adilson Gonzaga por ter me recebido e guiado nesta jornada acadêmica. Espero poder contribuir à ciência com o mesmo profissionalismo e entusiasmo que me transmitiu.

Aos meus amados pais Regina e Batista, por todo esforço realizado, especialmente quanto à minha formação.

À minha tia-mãe Socorro, que me acompanha e apoia sempre.

Aos meu irmãos, Paula e Rafael, grandes exemplos de persistência e humildade.

Ao meu noivo Ailton, por todo amor e companheirismo.

Às professoras Silvana Costa e Suzete Correia, que despertaram em mim o interesse pela pesquisa.

Aos amigos do LAVI, pelas contribuições e momentos de descontração no laboratório.

Aos amigos do "Grupo da Comida", "Brunets", "RGEM e agregados" e da ZWEI, que alegraram meus dias em São Carlos.

Aos funcionários da pós-graduação do departamento de Engenharia Elétrica.

À FAPESP, CAPES e CNPq, pelo suporte financeiro.

À Universidade de São Paulo. 

"O sonho é que leva a gente para a frente.

Se a gente for seguir a razão, fica aquietado, acomodado."

(Ariano Suassuna) 



\section{RESUMO}

VIEIRA, Raissa Tavares. Descritores robustos à rotação de texturas baseados na abordagem LMP com acréscimo da informação de Magnitude e Sinal. 2017. 152p. Tese (Doutorado em Ciências) - Escola de Engenharia de São Carlos, Universidade de São Paulo, São Carlos, 2017.

Classificação de imagens de textura, especialmente aquelas com mudanças significativas de rotação, iluminação, escala e ponto de vista, é um problema fundamental e desafiador na área de visão computacional. Esta tese propõe dois descritores de imagem simples, porém eficientes, chamados de Sampled Local Mapped Pattern Magnitude (SLMP_M) e Completed Local Mapped Pattern (CLMP) aplicados na classificação de textura. Os descritores propostos são parte de um aprimoramento do descritor Local Mapped Pattern (LMP) para trabalhar de maneira eficiente com imagens de textura rotacionadas. Os métodos propostos necessitam de um pré-ajuste de parâmetros que utiliza o método de otimização por enxame de partículas, e são discriminativos e robustos para a descrição de texturas rotacionadas em ângulos arbitrários. Para a validação dos descritores propostos duas bases de imagens são utilizadas, Kylberg Sintorn Rotation Dataset e Brodatz Texture Rotation Dataset, uma nova base de dados desenvolvida pela autora, formada por imagens de texturas rotacionadas do Álbum de Brodatz. As duas bases contêm imagens de texturas naturais que foram rotacionadas fisicamente no momento da captura e rotacionadas por processos computacionais. É feita também uma avaliação da influência de métodos de interpolação no processo de rotação das imagens e são comparados com diferentes descritores presentes na literatura. Cinco métodos de interpolação são investigados: Lanczos, B-spline, Cúbica, Linear e Nearest Neighbor. Os resultados experimentais demonstram que os descritores propostos nesta tese superam o desempenho dos descritores Completed Local Binary Pattern (CLBP), e dos descritores que combinam a versão generalizada das características de Fourier com variações do descritor Local Binary Pattern (LBP), $\mathrm{LBP}^{\mathrm{DFT}}$, ILBP ${ }^{\mathrm{DFT}}, \mathrm{LTP}^{\mathrm{DFT}}$ e ILTP ${ }^{\mathrm{DFT}}$. Os resultados também demonstram que a escolha do método de interpolação no processo de rotação das imagens influencia na capacidade de reconhecimento.

Palavras-chave: Classificação de textura. Invariância à rotação. Descritor de micropadrão. 



\begin{abstract}
VIEIRA, Raissa Tavares. Texture descriptors robust to rotation based on the LMP approach by adding Magnitude and Signal information. 2017. 152p. Tese (Doutorado em Ciências) Escola de Engenharia de São Carlos, Universidade de São Paulo, São Carlos, 2017.

Texture image classification, especially those with significant changes of rotation, illumination, scale and point of view, is a fundamental and challenging problem in the field of computer vision. This thesis proposes two simple, but efficient, image descriptors called Sampled Local Mapped Pattern Magnitude (SLMP_M) and Completed Local Mapped Pattern (CLMP) applied in texture classification. The proposed descriptors are part of an enhancement to the Local Mapped Pattern (LMP) descriptor to work efficiently with rotated texture images. The descriptors proposed requires a parameter preset by the particle swarm optimization method, they are discriminating and robust for the description of rotated textures at arbitrary angles.. For the validation of the proposed descriptors two image datasets are used: Kylberg Sintorn Rotation Dataset and Brodatz Texture Rotation Dataset, a new texture dataset introduced, which contains rotated texture images from Brodatz's Album. Both databases contain images of natural textures that have been rotated by Hardware and computational procedures. An evaluation of the influence of interpolation methods on the image rotation process is also presented and compared with different descriptors in the literature. Five interpolation methods are investigated: Lanczos, B-spline, Cubic, Linear and Nearest Neighbor. The experimental results show that the descriptors proposed in this thesis outperform the performance of the Completed Local Binary Pattern (CLBP) descriptors, and the descriptors that combine the generalized version of the Fourier characteristics with variations of the descriptor Local Binary Pattern (LBP), LBPDFT, ILBDFT, LTPDFT e ILTPDFT compared. The results also prove that the selection of the interpolation method in the image rotation process influences the recognition capability.
\end{abstract}

Keywords: Texture classification. Rotation invariance. Micropattern descriptor. 



\section{LISTA DE ILUSTRAÇÕES}

FigurA 2. 1 ViZINHANÇAS CIRCULARES $(8,1),(16,2)$ E $(8,2)$ DE UM PIXEL EM MULTIRESOLUÇÃO

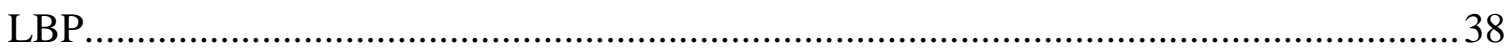

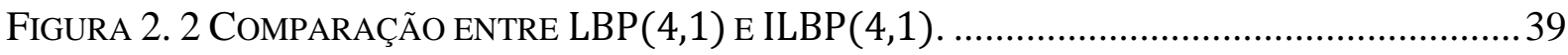

FIGURA 2. 3 COMPARATIVO DOS PADRÕES BINÁRIOS DO LBP E CS-LBP PARA UMA VIZINHANÇA DE 8 PIXELS 40

FIGURA 2. 4 (A) MICROPADRÃO DE 3X3 PIXELS (B) DIFERENÇAS LOCAIS (C) COMPONENTES DE SINAL (D) COMPONENTES DE MAGNITUDE.

FiguRA 2. 5 CÁLCULO DO HISTOGRAMA DE ORIENTAÇÃO DOS GRADIENTES DE UMA CÉLULA 8 X 8

FIGURA 2. 6 ACUMULADO DOS HISTOGRAMAS DE ORIENTAÇÃO DOS GRADIENTES EM BLOCOS PARA CRIAR A SAÍDA F1 DO DESCRITOR HOG PARA A JANELA DE 64 X 128 PIXELS DA IMAGEM $^{2}$ 44

FIGURA 2. 7 PADRÃO LOCAL DAS DIFERENÇAS DOS NÍVEIS DE CINZA...................................... 45

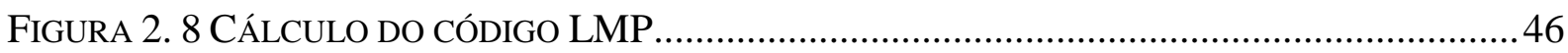

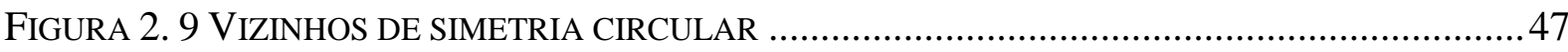

FIGURA 4. 1 DIFERENTES MICROPADRÕES COM MESMO CÓDIGO LBP GERADOS .......................57

FIGURA 4. 2 DIFERENTES MICROPADRÕES COM DIFERENTES CÓDIGOS LMP GERADOS...............58

FIGURA 4. 3 REPRESENTAÇÃO ESTRUTURAL E DE INTENSIDADE DE UM MICROPADRÃO DE 3 X 3 PIXELS.

FigurA 5. 1DiAgramA DE FlUXO DO ALGORITMO PSO PARA AJUSTE DOS PARÂMETROS DO LMP 67

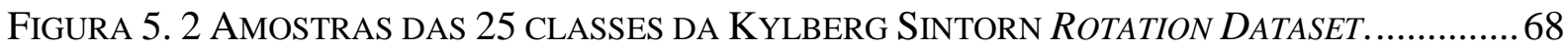

FIGURA 5. 3 TRANSFERIDOR COM EIXO GIRATÓRIO............................................................. 70

FIGURA 5. 4 ILUSTRAÇÃO DA DISTÂNCIA ENTRE A LENTE DA CÂMERA E A TEXTURA..................70

FigURA 5. 5 BASE DE IMAGENS COM 32 TEXTURAS DO ÁlBUM DE BRODATZ........................... 71

FIGURA 5. 6 EXEMPLO DE DIGITALIZAÇÃO DE TEXTURA EM 21 ÂNGULOS DE ROTAÇÃO...............72

FIGURA 5. 7 EXTRAÇÃO DE AMOSTRAS ALEATÓRIAS DA IMAGEM DE TEXTURA ROTACIONADA. 72

FIGURA 5. 8 PROCESSO DE CLASSIFICAÇÃO. ..................................................................... 74

FIGURA 6. 1 ACURÁCIA MÉDIA E DESVIO PADRÃO PARA IMAGEM ROTACIONADA POR HARDWARE 
Figura 6. 2 ACURÁCIA MÉdiA E DESVIO PADRÃO PARA IMAGEM ROTACIONAdA PELA

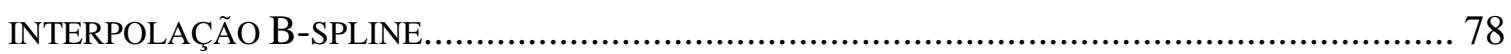

FIGURA 6. 3 ACURÁCIA MÉDIA E DESVIO PADRÃO PARA IMAGEM ROTACIONADA PELA

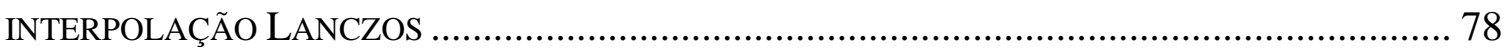

FIGURA 6. 4 ACURÁCIA MÉDIA E DESVIO PADRÃO PARA IMAGEM ROTACIONADA PELA

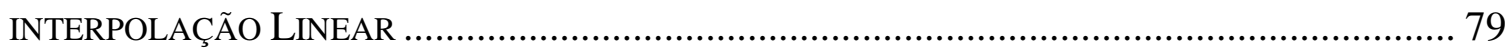

FiguRA 6. 5 ACURÁCIA MÉdiA E DESVIO PADRÃO PARA IMAGEM ROTACIONADA PELA

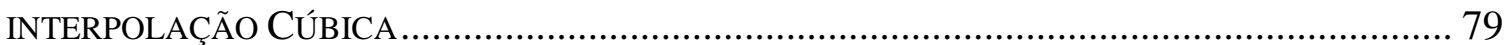

FIGURA 6. 6 ACURÁCIA MÉDIA E DESVIO PADRÃO PARA IMAGEM ROTACIONADA PELA INTERPOLAÇÃO NEAREST NEIGHBOR 80

Figura 6. 7 AVALIAÇÃO DOS DESCRITORES COM DifERENTES (P,R) EM IMAGENS ROTACIONADAS POR HARDWARE 81

Figura 6. 8 AVALIAÇÃO DOS DESCRITORES COM DIFERENTES (P,R) EM IMAGENS ROTACIONADAS POR INTERPOLAÇ̃̃O B-SPLINE... 81

Figura 6. 9 AVALIAÇÃO DOS DESCRITORES COM DIFERENTES (P,R) EM IMAGENS ROTACIONADAS POR INTERPOLAÇÃO LANCZOS 82

Figura 6. 10 AVALIAÇÃO DOS DESCRITORES COM DIFERENTES (P,R) EM IMAGENS ROTACIONADAS POR INTERPOLAÇÃO CÚBICA 83

Figura 6. 11 AVALIAÇÃO DOS DESCRITORES COM DIFERENTES (P,R) EM IMAGENS ROTACIONADAS POR INTERPOLAÇÃO LINEAR 83

Figura 6. 12 AVALIAÇÃO DOS DESCRITORES COM DIFERENTES (P,R) EM IMAGENS ROTACIONADAS POR INTERPOLAÇÃO NEAREST 83

FIGURA 6. 13 ACURÁCIA MÉDIA DOS DESCRITORES COM MELHOR DESEMPENHO EM DIFERENTES TAMANHOS DE AMOSTRAS ROTACIONADAS POR HARDWARE 84

FIGURA 6. 14 ACURÁCIA MÉDIA DOS DESCRITORES COM MELHOR DESEMPENHO EM DIFERENTES TAMANHOS DE AMOSTRAS ROTACIONADAS PELA INTERPOLAÇÃO B-SPLINE 84

FIGURA 6. 15 ACURÁCIA MÉDIA DOS DESCRITORES COM MELHOR DESEMPENHO EM DIFERENTES TAMANHOS DE AMOSTRAS ROTACIONADAS PELA INTERPOLAÇÃO LANCZOS 85

FIGURA 6. 16 ACURÁCIA MÉDIA DOS DESCRITORES COM MELHOR DESEMPENHO EM DIFERENTES TAMANHOS DE AMOSTRAS ROTACIONADAS PELA INTERPOLAÇÃO LINEAR 86

FIGURA 6. 17 ACURÁCIA MÉDIA DOS DESCRITORES COM MELHOR DESEMPENHO EM DIFERENTES TAMANHOS DE AMOSTRAS ROTACIONADAS PELA INTERPOLAÇÃO CUBIC. 86

FIGURA 6. 18 ACURÁCIA MÉDIA DOS DESCRITORES COM MELHOR DESEMPENHO EM DIFERENTES TAMANHOS DE AMOSTRAS ROTACIONADAS PELA INTERPOLAÇÃO NEAREST NEIGHBOR 86 
FIGURA 6. 19 ACURÁCIA MÉDIA DO DESCRITOR SLMP_M (24,3) PARA DIFERENTES MÉTODOS DE

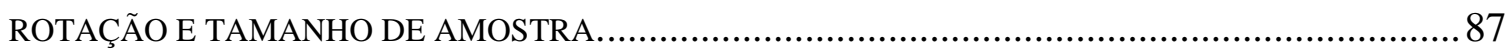

FiguRA 6. 20 ACURÁCIA MÉDIA DO DESCRITOR CLMP PARA DIFERENTES MÉTODOS DE

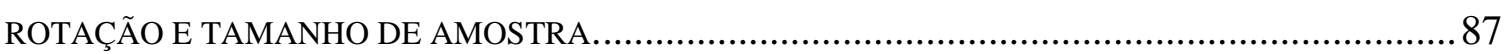

FIGURA 6. 21 ACURÁCIA MÉDIA DO DESCRITOR CLBP PARA DIFERENTES MÉTODOS DE ROTAÇÃO

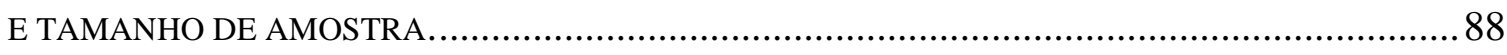

FIGURA 6. 22 MATRIZ DE CONFUSÃO DO DESCRITOR CLMP_S/M $(23,4)$................................ 91

FIGURA 6. 23 EXEMPLO DE TEXTURAS CLASSIFICADAS INCORRETAMENTE PELO DESCRITOR

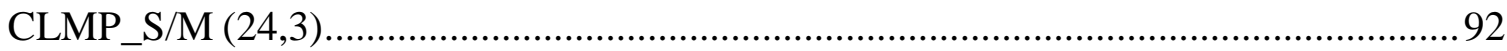

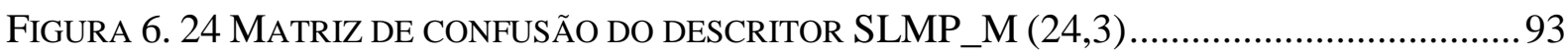

FIGURA 6. 25 EXEMPLO DE TEXTURAS CLASSIFICADAS INCORRETAMENTE PELO DESCRITOR

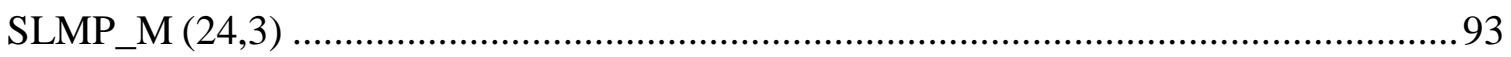

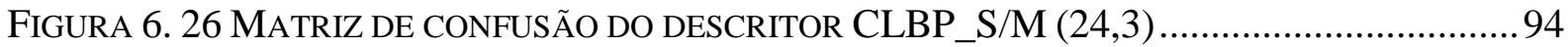

FIGURA 6. 27 EXEMPLO DE TEXTURAS CLASSIFICADAS INCORRETAMENTE PELO DESCRITOR

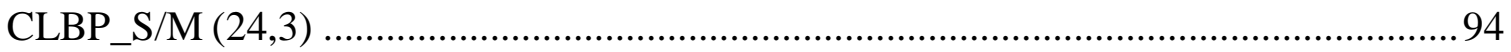





\section{LISTA DE TABELAS}

TABELA 4. 1 COMPARAÇÃO DOS VETORES DE CARACTERÍSTICAS DO CLMP E CLBP ................62

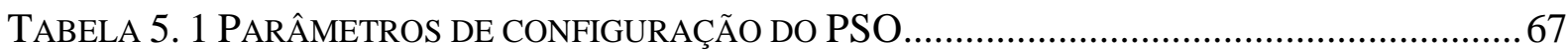

TABELA 6. 1 PARÂMETROS ÓTIMOS PARA A BASE KYLBERG SinTORN....................................... 75

TABela 6. 2 ParÂMETRos ÓtIMOS PARA A BASE Brodatz TEXTURE Rotation DATASET ......... 76

TABELA 6. 3 DiMENSÃO DOS DESCRITORES AVALIADOS COM CONFIGURAÇÃO $(8,1) \ldots \ldots \ldots \ldots \ldots . . . . . . .77$

TABELA 6. 4 DIMENSÃO DOS DESCRITORES AVALIADOS COM DIFERENTES CONFIGURAÇÕES DE

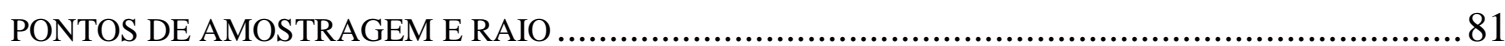

TABELA 6. 5 TEMPO COMPUTACIONAL PARA GERAÇÃO DO VETOR DE CARACTERÍSTICAS..........90 



\section{LISTA DE SIGLAS}

\begin{tabular}{|c|c|}
\hline $1-\mathrm{NN}$ & Fisrt Nearest Neighbor \\
\hline BS & B-spline \\
\hline CS-LBP & Center-Symmetric Local Binary Pattern \\
\hline CS-LMP & Center-Symmetric Local Mapped Pattern \\
\hline CLBP & Completed Local Binary Pattern \\
\hline CLBP_C & Completed Local Binary Pattern_Center \\
\hline CLBP_S & Completed Local Binary Pattern_Sign \\
\hline CLBP_M & Completed Local Binary Pattern_Magnitude \\
\hline CLMP & Completed Local Mapped Pattern \\
\hline CLMP_C & Completed Local Mapped Pattern_Center \\
\hline CLMP_S & Completed Local Mapped Pattern_Sign \\
\hline CLMP_M & Completed Local Mapped Pattern_Magnitude \\
\hline $\mathrm{CB}$ & Cúbica de terceira ordem \\
\hline GLCM & Gray Level Co-occurrence Matrix \\
\hline HOG & Histograms of Oriented Gradients \\
\hline HW & Hardware \\
\hline ILBP & Improved Local Binary Pattern \\
\hline ILBPDFT & Improved Local Binary Pattern Discrete Fourier Transform \\
\hline ILTP & Improved Local Ternay Pattern \\
\hline ILTPDFT & Improved Local Ternary Pattern Discrete Fourier Transform \\
\hline & Laboratório de Visão Computacional do Depto. de Engenharia Elétrica \\
\hline Let & EESC-USP \\
\hline LBP & Local Binary Pattern \\
\hline $\mathrm{LBP}_{\mathrm{P}, \mathrm{R}}^{\mathrm{ri}}$ & Local Binary Pattern Rotation Invariant \\
\hline $\mathrm{LBP}_{\mathrm{P}, \mathrm{R}}^{\mathrm{riu} 2}$ & Local Binary Pattern Rotation Invariant Uniform \\
\hline LBPDFT & Local Binary Pattern Discrete Fourier Transform \\
\hline LBPHF & Local Binary Pattern Histogram Fourier \\
\hline LDSMT & Local Difference Sign-Magnitude Transform \\
\hline LFP & Local Fuzzy Pattern \\
\hline LMP & Local Mapped Pattern \\
\hline
\end{tabular}




$\begin{array}{ll}\text { LN } & \text { Linear } \\ \text { LTP } & \text { Local Ternary Pattern } \\ \text { LTPDFT } & \text { Local Ternary Pattern Discrete Fourier Transform } \\ \text { LZ } & \text { Lanczos } \\ \text { NN } & \text { Nearest Neighbor } \\ \text { PSO } & \text { Particle Swarm Optimization } \\ \text { PCA } & \text { Principal Component Analysis } \\ \text { S-LMP } & \text { Sampled Local Mapped Pattern } \\ \text { SLMP_M } & \text { Sampled Local Mapped Pattern Magnitude } \\ \text { TU } & \text { Texture Unit }\end{array}$




\section{SUMÁRIO}

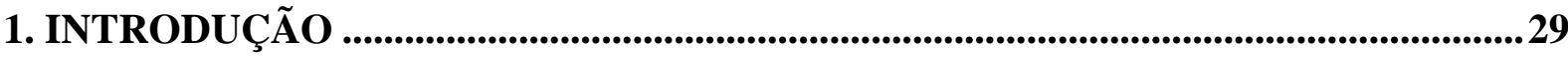

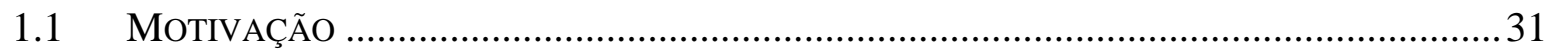

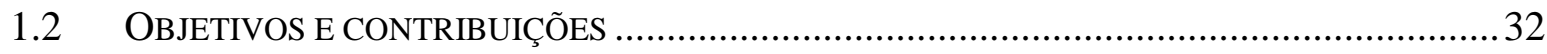

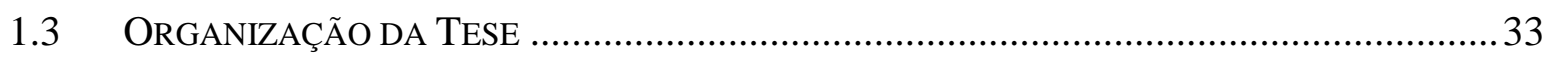

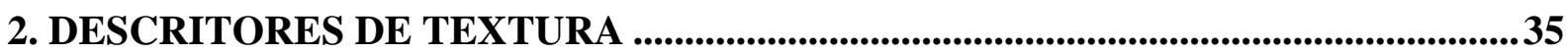

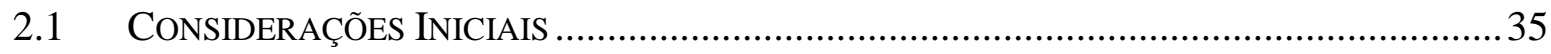

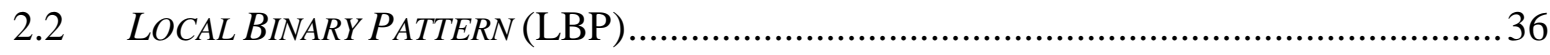

2.3 LOCAL BINARY PATTERN ROTATION INVARIANT (LBP $\mathrm{P}, \mathrm{R})$...................................... 37

2.4 LOCAL BINARY PATTERN ROTATION INVARIANT UNIFORM $\left(\mathrm{LBP}_{\mathrm{P}, \mathrm{R}}^{\mathrm{RIU} 2}\right)$....................... 38

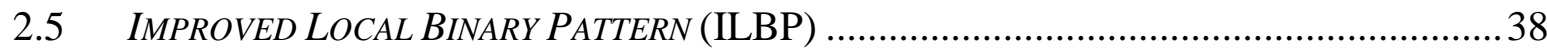

2.6 CENTER-SYMMETRIC LOCAL BINARY PATTERN (CS-LBP) ......................................... 40

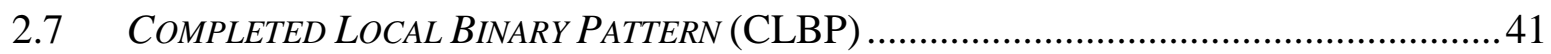

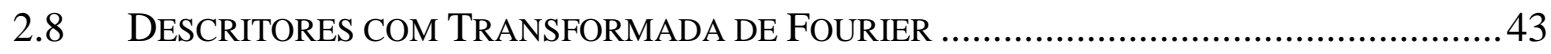

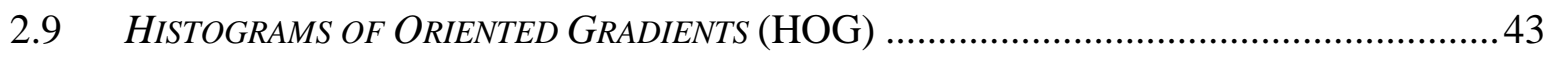

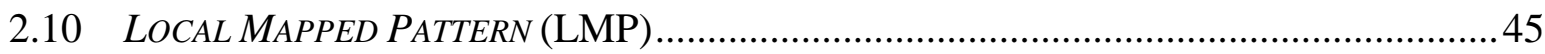

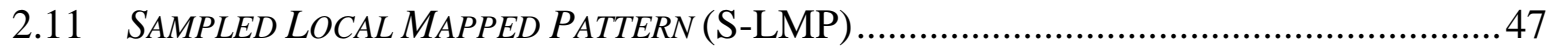

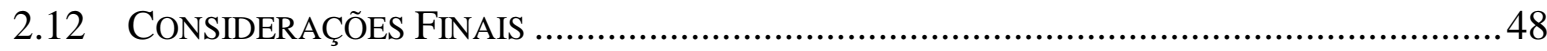

3. OTIMIZAÇÃO POR ENXAME DE PARTÍCULAS ....................................................... 49

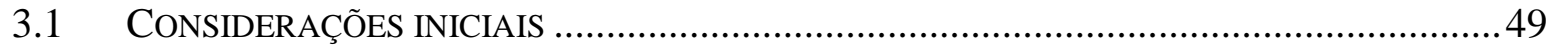

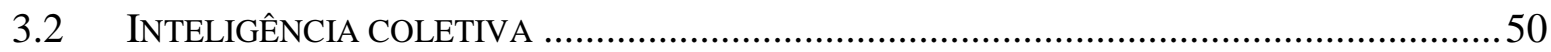

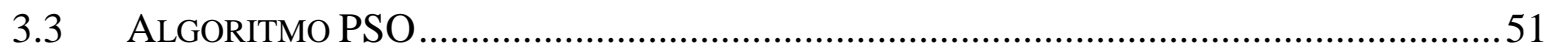

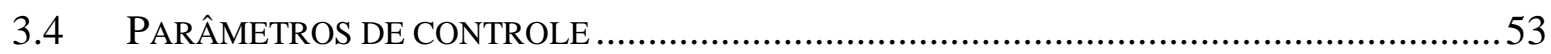

3.5 VARIAÇÕES E MELHORIAS DOS COMPONENTES DO PSO .........................................54

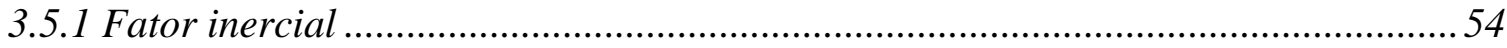

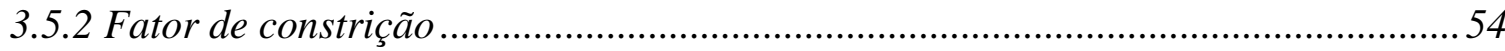

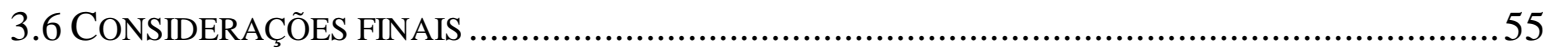

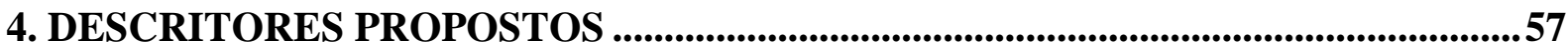

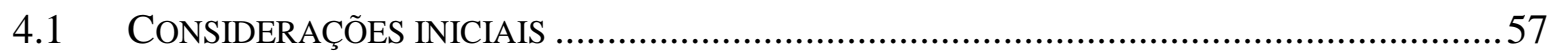

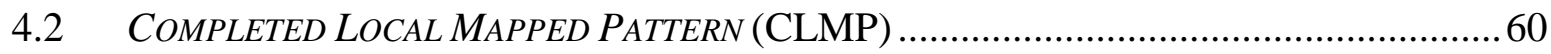

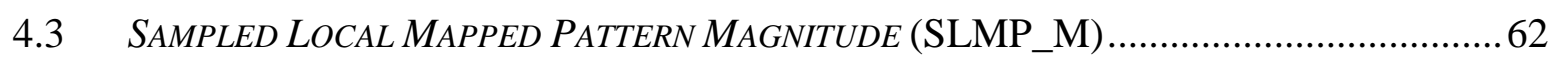




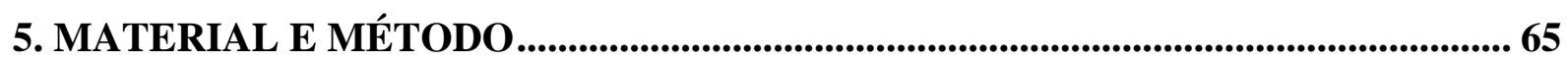

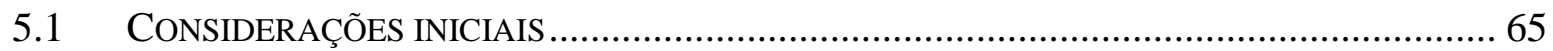

5.2 OTIMIZAÇÃO DOS PARÂMETROS $\beta$ E $B$ DOS DESCRITORES BASEADOS NO MÉTODO LMP 65

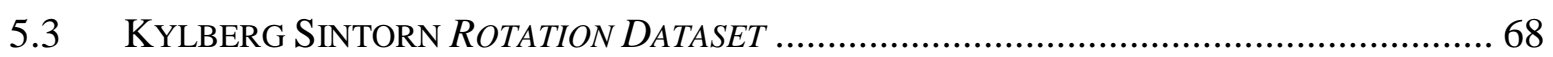

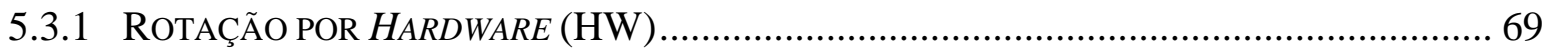

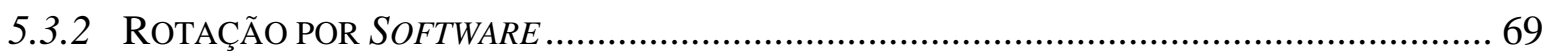

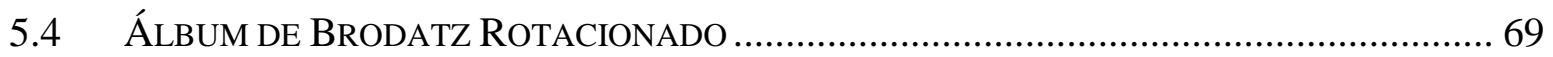

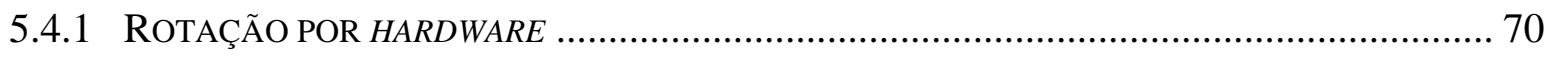

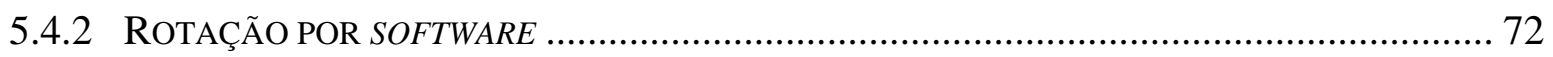

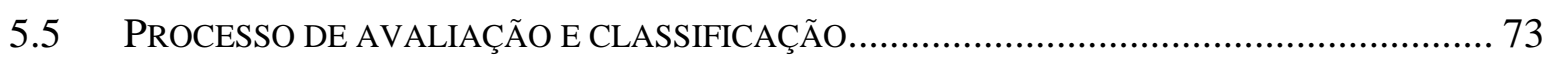

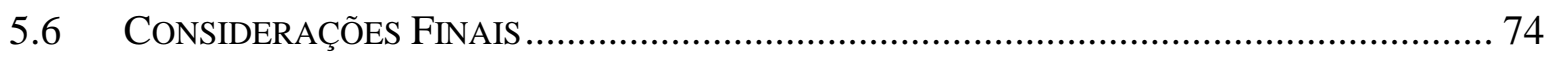

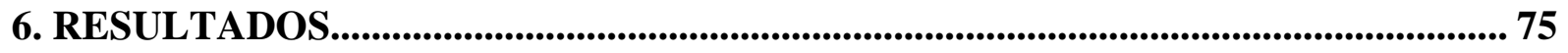

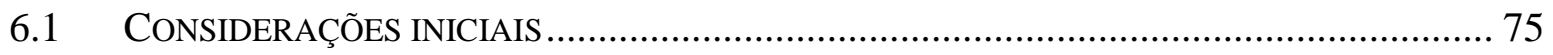

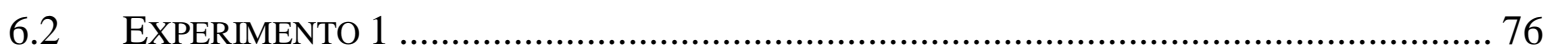

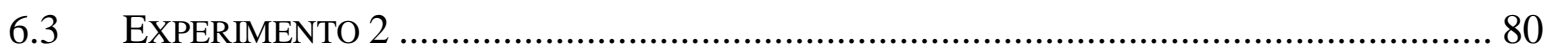

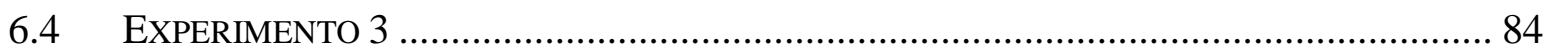

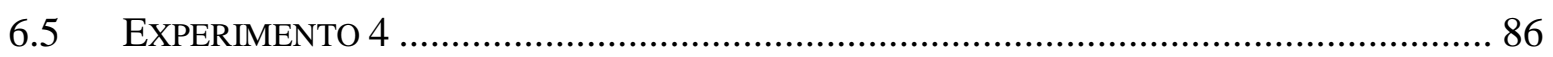

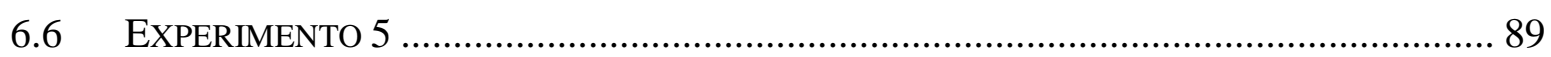

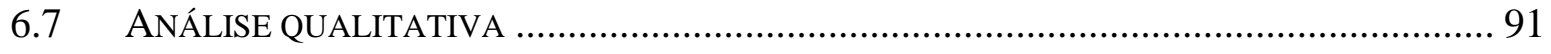

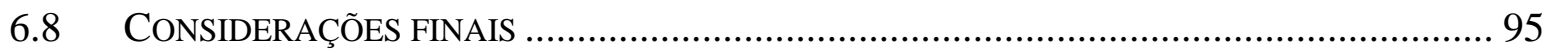

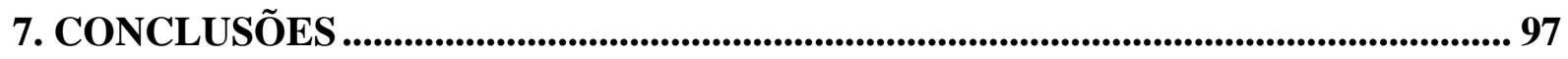

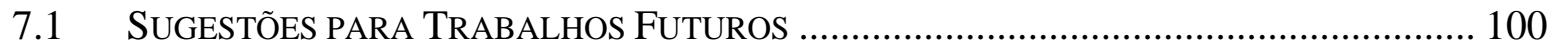

7.2 PUBLICAÇÕES ORIUNDAS DO TRABALHO ............................................................. 100

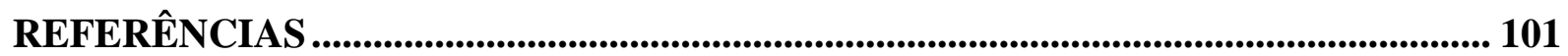

APÊNDICE A - ACURÁCIAS MÉdIAS OBTIDAS PELOS DESCRITORES AVALIADOS NO EXPERIMENTO 1 ................................................................. 111

APÊNDICE B - ACURÁCIAS MÉDIAS OBTIDAS PELOS DESCRITORES AVALIADOS NO EXPERIMENTO 2 ........................................................................... 115 
APÊNDICE C - ACURÁCIAS MÉDIAS OBTIDAS PELOS DESCRITORES AVALIADOS NO EXPERIMENTO 3 .................................................................................119 APÊNDICE D - ACURÁCIAS MÉDIAS OBTIDAS PELOS DESCRITORES AVALIADOS NO EXPERIMENTO 4 ....................................................................................129 



\section{Capítulo 1}

\section{Introdução}

A extração de características de textura está presente em uma variedade de aplicações da visão computacional, tais como classificação (NGUYEN et al., 2016; PAN et al., 2017), reconhecimento de face (LATIF et al., 2016; HU et al., 2016), correspondência de imagens (FERRAZ; GONZAGA, 2016; SONG et al., 2017a;), reconhecimento de cenas e objetos (REN; JIANG; YUAN, 2017; SHANG et al., 2016), e diagnóstico médico (DAS et al., 2015; MORALES et al., 2017) entre outras.

No mundo real, as texturas além de apresentarem padrões intrínsecos complexos que são difíceis de descrever ao usar um modelo universal, também exibem grandes variações extrínsecas como, por exemplo, a não uniformidade, mudanças de orientação, escala e iluminação, devido a diferentes condições da imagem. A maioria das abordagens utilizadas para classificação de textura assume, explícita ou implicitamente, que as amostras desconhecidas a serem classificadas são idênticas às amostras de treinamento com relação à escala espacial, orientação e valores em escala de cinza. No entanto, texturas do mundo real podem ocorrer em resoluções espaciais e rotações arbitrárias e podem estar sujeitas a diferentes condições de iluminação. Em tal cenário, em uma boa representação das características de uma imagem esperam-se as seguintes propriedades para uma classificação eficiente de texturas (SONG et al., 2017b):

- Discriminativa, para distinguir diferentes classes de texturas;

- Invariante às transformações da imagem, tais como: rotação, iluminação, escala e mudança de ponto de vista;

- Insensível ao ruído;

- Baixa dimensionalidade, para facilitar operações subsequentes (por exemplo, armazenamento, correspondência ou classificação);

- Eficiente para implementação.

O desafio de uma classificação eficiente inspirou uma coleção de estudos, que geralmente incorporam invariância em relação a uma ou no máximo duas das propriedades de 
escala espacial, orientação e escala de cinza. Kashyap e Khotanzad (1986) estão entre os primeiros pesquisadores a estudar a classificação de textura invariante à rotação usando um modelo auto regressivo circular.

$\mathrm{Na}$ fase inicial, muitos modelos foram explorados para estudar invariância à rotação para a classificação de textura, como os modelos ocultos de Markov (CHEN; KUNDU, 1994). Varma e Zisserman (2005) propuseram um conjunto de treinamento formado por um dicionário de textons invariante à rotação, e, em seguida, as imagens de textura foram classificadas com base na distribuição desses textons. A análise fractal também foi proposta para classificação de texturas invariantes à rotação no trabalho de Varma e Garg (2007).

Também foram propostas algumas técnicas para estimar a orientação da imagem de maneira a facilitar a classificação das texturas, incluindo métodos baseados na distribuição angular de potência do sinal no domínio de Fourier (BIGUN; GRANLUND; WIKLUND, 1991; CHANDRA, 1998), em gradientes de imagem e na estrutura de auto correlação do sinal (MESTER, 2000). No trabalho de Jafari-Khouzani e Soltanian-Zadeh (2005) a direção principal da textura é estimada ao aplicar a transformada Radon, a imagem é rotacionada para posicionar a direção principal em 0 graus. Em seguida, a transformada wavelet é utilizada para extrair características de textura.

Diante do desafio de rotações arbitrárias, ao longo dos anos foram propostos também vários descritores com propriedades invariantes à rotação com formulações mais simples para caracterização de imagens de textura, especialmente descritores baseados na codificação de micropadrões presentes nas texturas. Dentre os descritores de micropadrões existentes o Local Binary Pattern (LBP) proposto por Ojala, Pietikäinen e Mäenpää (2002), ganhou grande atenção de vários pesquisadores e seu algoritmo foi estendido para diferentes aplicações devido a sua simplicidade de implementação (HEIKKILÄ; PIETIKÄINEN; SCHMID, 2009; LATEGAHN et al., 2010). O LBP e suas variações representam a relação de cada pixel e os seus vizinhos (localizado em um círculo em torno do pixel) por um padrão binário e utilizam o histograma dos padrões de textura para a classificação.

Em trabalho recente, Liu et al. (2017) apresentam um levantamento das variações do LBP com mais destaque na literatura, dentre elas o Completed Local Binary Pattern (CLBP) (GUO; ZHANG; ZHANG, 2010a) que utiliza vizinhanças circulares e foi proposto para trabalhar com imagens rotacionadas realizando a combinação de vários descritores para gerar seu vetor final de características. Devido ao seu desempenho na descrição de texturas e a simplicidade de implementação, o CLBP está presente em vários trabalhos recentes. Zeppelzauer et al. (2016) aplicam esse descritor na classificação de textura em imagens de 
rochas. Rassem (2017) apresenta uma extensão do CLBP para imagens coloridas aplicado na categorização de imagens de cenas de eventos esportivos. Os autores Ryu, Hong e Yang (2015) propuseram uma extensão do CLBP para classificação de texturas. Chen et al. (2016) também apresentaram uma outra extensão do CLBP; os autores aprimoraram o seu poder de representação com uma versão multiescala aplicada na classificação de imagens de sensoriamento remoto.

Apesar da popularidade dos descritores baseados no LBP, o método convencional de codificação invariante à rotação tem a desvantagem de negligenciar a informação de alguns padrões durante seu procedimento de codificação. E é neste contexto que esta tese está inserida, pois, os padrões desconsiderados, também possuem poder discriminativo e, por meio da evolução do descritor Local Mapped Pattern (LMP) proposta nesta tese, é possível obter robustez na descrição de imagens rotacionadas, com menos perda de informação dos padrões contidos na imagem digitalizada.

\subsection{Motivação}

Apesar da existência de várias abordagens para descrição de imagens de texturas, não há um método genérico ou formal que seja válido para vários tipos de imagens e aplicações. As variadas definições de textura conduzem a diferentes formas de análise, assim, várias abordagens foram desenvolvidas buscando utilizar a textura como base na descrição de uma imagem rotacionada. No entanto, a maioria dos métodos ainda codifica apenas um tipo de informação, por exemplo, a intensidade ou a direção, o que limita as suas capacidades de descrição.

Como o Local Mapped Pattern (LMP), um descritor de texturas em desenvolvimento no grupo de pesquisa do Laboratório de Visão Computacional (LAVI), tem obtido resultados eficientes na classificação de texturas gerais e, a sua formulação paramétrica permitir diferentes configurações, é motivação deste trabalho propor uma nova abordagem, que seja mais eficiente para a descrição de texturas rotacionadas, baseada no descritor paramétrico LMP.

Em algumas aplicações, como por exemplo, reconhecimento de face, a orientação do objeto é conhecida, enquanto que em muitas outras aplicações a orientação de um objeto pode ser arbitrária e, portanto, também a textura. No último caso, a textura pode ser girada para uma orientação principal ou direção principal, ou, alternativamente, um descritor de textura invariante à rotação pode ser usado. 
Para validação de descritores de textura invariantes à rotação é necessário realizar testes em imagens com diferentes ângulos de rotação. Na literatura é possível encontrar poucas opções de bases com imagens de texturas rotacionadas; na Tabela 1.1 são detalhadas três das bases mais usadas atualmente.

Tabela 1. 1 Descrição das bases de imagem de textura

\begin{tabular}{|c|c|c|}
\hline Base de imagens & Classes & Ângulos de rotação \\
\hline $\begin{array}{l}\text { Outex } \\
\text { (OJALA et al., 2002) }\end{array}$ & 9 & $0^{\circ} 5^{\circ} 10^{\circ} 15^{\circ} 30^{\circ} 45^{\circ} 60^{\circ} 75^{\circ} 90^{\circ}$ \\
\hline $\begin{array}{l}\text { Mondial Marmi } \\
\text { (BIANCONI, 2012) }\end{array}$ & 12 & $0^{\circ} \quad 10^{\circ} 20^{\circ} 30^{\circ} 40^{\circ} 50^{\circ} 60^{\circ} 70^{\circ} 80^{\circ} 90^{\circ}$ \\
\hline $\begin{array}{l}\text { Kylberg Sintorn Rotation dataset } \\
\text { (KYLBERG, 2014) }\end{array}$ & 25 & $0^{\circ} 40^{\circ} 80^{\circ} \quad 120^{\circ} \quad 160^{\circ} 200^{\circ} 240^{\circ} 280^{\circ} 320^{\circ}$ \\
\hline
\end{tabular}

Observa-se que não há uma grande variação dos ângulos de rotação, bem como a quantidade de classes disponíveis. Assim, é motivação deste trabalho desenvolver e disponibilizar uma nova base com mais classes e ângulos de rotação de maneira a aproximar de situações reais em que é possível encontrar imagens nos mais variados ângulos de rotação. Dentre as bases citadas, a base Kylberg Sintorn além de contar com imagens rotacionadas via hardware, também apresenta imagens rotacionadas por métodos computacionais. Por se tratar da única base com imagens rotacionadas via software, também é motivação deste trabalho apresentar uma nova opção de base de imagens de texturas rotacionadas por métodos computacionais com mais opções de ângulos e diferentes classes.

A avaliação de técnicas de interpolação no processo de rotação de imagens mostra-se importante em métodos que necessitam realizar rotações computacionais durante seu processo de codificação da imagem como é realizado, por exemplo, no trabalho de Jafari-Khouzani e Soltanian-Zadeh (2005).

\subsection{Objetivos e contribuições}

O principal objetivo desta tese de doutoramento é o desenvolvimento de descritores de textura que codifiquem as imagens submetidas a rotações em ângulos arbitrários de maneira robusta, baseados no descritor Local Mapped Pattern (LMP). Além disso, a obtenção dos parâmetros inerentes ao método LMP devem ser obtidos de maneira automática, simples e 
confiável e não de maneira empírica como realizado até então. Assim, também é objetivo deste trabalho apresentar um algoritmo de otimização por enxame de partículas (PSO) (KENNEDY; EBERHART, 1995), como ferramenta de otimização, mostrando a sua viabilidade na busca de parâmetros ótimos para os descritores baseados no descritor Local Mapped Pattern no processo de classificação de texturas.

Ainda mais, é objetivo desta tese verificar se rotações realizadas por meio de métodos computacionais de interpolação (por software) podem ser usadas para se avaliar texturas rotacionadas em contraposição a texturas adquiridas já rotacionadas (por hardware).

A primeira contribuição desta tese foi o desenvolvimento de uma nova base rotacionada do Álbum de Brodatz, com maior quantidade de amostras, diferentes tamanhos de amostra e ângulos de rotação. Esta nova base contém imagens rotacionadas por Hardware como também, por cinco métodos computacionais de interpolação: Lanczos, B-spline, Cúbica, Linear e Nearest Neighbor.

A segunda contribuição foi o desenvolvimento do descritor Completed Local Mapped Pattern (CLMP), que realiza a combinação de três diferentes operadores: CLMP_M, CLMP_S e CLMP_C para codificar a informação em imagens de texturas. Este descritor é inspirado no Completed Local Binary Pattern (CLBP), mas, diferente deste, com a mesma maneira de combinação dos operadores possibilita uma excelente acurácia média no processo classificatório para diferentes configurações e testes experimentais.

A terceira contribuição foi o desenvolvimento do descritor Sampled Local Mapped Pattern Magnitude (SLMP_M). O descritor combina a informação de sinal do descritor Sampled Local Mapped Pattern (SLMP), que é uma versão de vizinhança circular do descritor LMP, com a inserção da informação de magnitude de maneira concatenada. O descritor SLMP_M é uma variação do primeiro descritor proposto nesta tese.

\subsection{Organização da Tese}

Estruturalmente, este trabalho está dividido da seguinte maneira:

O Capítulo 1 é a introdução ao assunto abordado neste trabalho, motivação, objetivos e contribuições e organização desta tese.

O Capítulo 2 apresenta as principais abordagens utilizadas para descrição de textura. 
No Capítulo 3 é apresentado o formalismo do método de otimização baseado em enxame de partículas. Inicialmente, são introduzidos os conceitos e definições e é apresentado o equacionamento que descreve o modelo do algoritmo.

No Capítulo 4 são formulados os métodos propostos nesta tese para descrição de imagens de textura rotacionadas.

A metodologia empregada neste trabalho para otimização na descrição de texturas está detalhada no Capítulo 5, bem como, o processo de digitalização de uma nova base de imagens rotacionadas do Álbum de Brodatz.

No Capítulo 6 são apresentados e discutidos os resultados obtidos nesta tese.

E finalmente, no Capítulo 7, são mostradas as conclusões, discutindo-se as limitações dos descritores propostos, sendo apresentadas sugestões de continuidade deste trabalho. 


\section{Capítulo 2}

\section{Descritores de textura}

\subsection{Considerações Iniciais}

Em visão computacional, descritores de padrões locais são baseados em padrões da imagem extraídos de pequenas regiões e usam histogramas para computar suas ocorrências e gerar um vetor de características. Vários descritores foram propostos nos últimos anos com o objetivo de caracterizar estes padrões de texturas locais.

Pode-se considerar a Matriz de Coocorrência de Níveis de Cinza (GLCM - Gray Level Co-occurrence Matrix) proposta por Haralick, Shanmugam, e Dinstein (1973) como o método pioneiro na caracterização de imagens de textura. Esse método considera a ocorrência estatística de cada nível de cinza em pixels diferentes na imagem definidos ao longo de diferentes direções. Uma vez criada a matriz GLCM, várias medidas texturais podem ser calculadas a partir dela: contraste, correlação, energia, diferença de entropia, entre outras. As matrizes de co-ocorrência são uma boa ferramenta na classificação de imagens, no entanto, apresentam alto custo computacional.

Motivados pela GLCM, He e Wang (1990) propuseram o Texture Unit (TU), método que considera uma vizinhança de $3 \times 3$ pixels da imagem para extração de informações. Comparando-se os valores de intensidade de nível de cinza, a unidade de textura padrão é gerada. Aos pixels da vizinhança que têm exatamente o mesmo valor que o pixel central é atribuído o valor um, aos pixels da vizinhança que têm valores menores que o pixel central, valor zero e aos pixels da vizinhança com valores maiores do que o pixel central atribui-se o valor dois. No entanto, na prática, apenas uma proporção muito pequena de pixels vizinhos têm exatamente os mesmos valores do pixel central. Portanto, a maioria das unidades de textura são zero ou dois, fazendo o TU parecer um padrão binário. A partir deste método é possível se obter até $3^{8}=6561$ códigos de unidades de textura.

Inspirados no método Texture Unit, Ojala, Pietikainen e Harwood (1996) propuseram o Local Binary Pattern (LBP) que assim como o Texture Unit, tem como ideia básica que uma imagem é composta por micropadrões e, ao longo dos anos, vários descritores baseados 
neste método foram publicados. Nas seções seguintes encontra-se um conjunto, embora incompleto, dos descritores mais usados na atualidade, como também novos desdobramentos baseados no descritor LMP, descritor de micropadrões em desenvolvimento no LAVI.

\subsection{Local Binary Pattern (LBP)}

No LBP uma vizinhança 3 x 3 pixels é analisada comparando-se a intensidade do nível de cinza do pixel central com os pixels da vizinhança utilizando-se uma função de limiar binária para extração dos padrões binários de acordo com o resultado da comparação. Formalmente, o LBP é definido pela Equação 2.1:

$$
L B P=\sum_{p=1}^{8} s\left(g_{p}-g_{c}\right) 2^{p-1} \quad s(x)= \begin{cases}1, & x \geq 0 \\ 0, & x<0\end{cases}
$$

na qual, $g_{c}$ é o valor do nível de cinza do pixel central e $g_{p}$ são os valores dos níveis de cinza dos pixels da vizinhança.

Como esta abordagem utiliza apenas dois níveis de limiarização há uma redução na quantidade de códigos gerados de 6561 do TU para 256 códigos do LBP, isto faz com que a técnica se torne mais rápida na classificação de texturas.

O LBP tornou-se um dos descritores mais utilizados na literatura, e tem sido aplicado com sucesso em muitas tarefas de visão computacional, tais como reconhecimento de face (AHONEN; HADID; PIETIKAINEN, 2006), classificação de textura (TOPI et al, 2000; LIAO; LAW; CHUNG, 2009; GUO; ZHANG; ZHANG, 2010a), detecção de forma (HUANG; LI; WANG, 2004), e a detecção humana (HE et al, 2007). Pietkäinen et al. (2011) apresentam uma compilação de vários trabalhos desenvolvidos a partir da aplicação do LBP.

Com o objetivo de lidar melhor com o ruído, particularmente em regiões quase uniformes da imagem, TAN e TRIGGS (2007) propuseram o Local Ternary Pattern (LTP) que estende os códigos do LBP para três valores, ou seja, a função binária $s(x)$ é substituída pela função ternária da Equação 2.2:

$$
s(x, t)=\left\{\begin{array}{rl}
1 & x \geq g_{c}+t \\
0 & x-g_{c}<t \\
-1 & x \leq g_{c}-t
\end{array}\right.
$$


Neste descritor a diferença entre os pixels da vizinhança $g_{p}$ e o valor de pixel central $g_{c}$ é codificada com três valores usando um valor de limiar $t$. Em vez de usar um código com base 3 para codificar os três estados, o LTP usa dois códigos binários representando os componentes positivo e negativo do código ternário. Isto é, dois códigos binários que codificam os dois estados $\{-1,1\}$. Esses códigos binários são armazenados em dois histogramas separados e, como último passo, os histogramas são concatenados para formar o vetor de características LTP.

\subsection{Local Binary Pattern Rotation Invariant $\left(\mathrm{LBP}_{\mathrm{P}, \mathrm{R}}^{\mathrm{ri}}\right)$}

Com o objetivo de tornar o LBP invariante à rotação e à variação de níveis de cinza Ojala, Pietikäinen e Mäenpää (2002) estenderam a definição do LBP para vizinhanças circulares do pixel, o Local Binary Pattern Rotation Invariant $\left(\mathrm{LBP}_{\mathrm{P}, \mathrm{R}}^{\mathrm{ri}}\right.$ ) que não impõe limite para o tamanho da vizinhança ou para o número de pontos de amostragem.

Nesta evolução do $L B P$, os pixels da vizinhança do pixel central são indexados de modo que formem uma cadeia circular (tornando mais fácil de obter descritores de texturas invariantes a rotação). Os pixels da vizinhança em posição diagonal são obtidos por meio de interpolação bilinear. Ambas as modificações são necessárias para obter a vizinhança circularmente simétrica, o que permite derivar para uma versão de rotação invariável. Se for aplicado $(P=8, R=1)$, obtém-se o $\mathrm{LBP}_{8,1}^{\text {ri }}$, que é similar ao LBP básico proposto em (OJALA; PIETIKAINEN; HARWOOD, 1996).

Considerando-se uma imagem $I$ uma matriz de níveis de cinza $I(x, y), g_{c}$ o nível de cinza de um pixel arbitrário de $I$ na coordenada $(x, y), g_{p}$ o nível de cinza de um ponto de amostragem em uma vizinhança circular com $P$ amostras igualmente espaçadas em um raio $R$ na vizinhança do ponto $(x, y)$, suas coordenadas podem ser calculadas pelas Equações 2.3, 2.4 e 2.5 .

$$
\begin{gathered}
g_{p}=I\left(x_{p}, y_{p}\right) \quad p=0, \ldots, P-1 \\
x_{p}=x+R \times \cos \left(\frac{2 \pi p}{P}\right) \\
y_{p}=y-R \times \sin \left(\frac{2 \pi p}{P}\right)
\end{gathered}
$$


A Figura 2.1 ilustra a composição da vizinhança circular para diferentes valores de $P$ e $R$, número de vizinhos e raio, respectivamente.

Figura 2.1 Vizinhanças circulares $(8,1),(16,2)$ e $(8,2)$ de um pixel em multiresolução LBP.

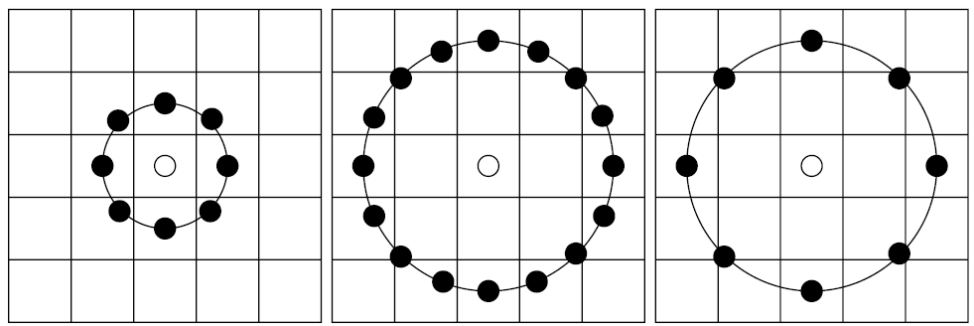

Fonte: Pietikainen et al. (2011).

\subsection{Local Binary Pattern Rotation Invariant Uniform $\left(\mathrm{LBP}_{\mathrm{P}, \mathrm{R}}^{\mathrm{riu} 2}\right)$}

Uma desvantagem do $\mathrm{LBP}_{\mathrm{P}, \mathrm{R}}^{\mathrm{ri}}$ é que o número possível de padrões locais cresce rapidamente se o número de vizinhos crescer, e dependendo da quantidade adotada, torna-se inviável para aplicações reais. Assim, Ojala, Pietikainen, Maenpaa (2002) propuseram outra extensão do LBP original utilizando os chamados padrões uniformes. Para isto, uma métrica de uniformidade de padrão é utilizada: $u$ é o número de transições bit a bit de 0 para 1 ou vice versa quando o padrão do bit é percorrido de maneira circular. Um código LBP é considerado uniforme se a medida de uniformidade $u$ é no máximo 2. Para exemplificar, os padrões 00000000 (zero transições), 01110000 (duas transições) e 11001111 (duas transições) são uniformes, enquanto os padrões 11001001 (quatro transições) e 01010010 (seis transições) não são.

Ojala, Pietikainen e Maenpaa (2002) notaram em seus experimentos com imagens de texturas, que padrões uniformes representam cerca de $90 \%$ de todos os padrões quando utiliza a vizinhança $(8,1)$ e cerca de $70 \%$ quando utiliza a vizinhança $(16,2)$. Com esta abordagem o número de códigos binários diferentes é reduzido, mantendo-se um bom desempenho. Por exemplo, o número dos bins do histograma com um LBP 8-bit é reduzido para 59 bins, sendo que os primeiros 58 bins são padrões uniformes e o último bin contém a frequência de ocorrência de todos os outros padrões não-uniformes (PIETIKÄINEN et al., 2011).

\subsection{Improved Local Binary Pattern (ILBP)}

Seguindo o LBP, Jin et al. (2004) propuseram o Improved Local Binary Pattern (ILBP), considerado mais robusto aos efeitos dos ruídos, porque o padrão local é gerado por meio da comparação das intensidades dos níveis de cinza de todos os pixels, incluindo o pixel 
central, com o valor médio das intensidades de nível de cinza da vizinhança. Portanto, o tamanho do descritor ILBP $\left(2^{9}\right)$ é duas vezes o tamanho do descritor LBP $\left(2^{8}\right)$. O ILBP é expresso pela Equação 2.6,

$$
I L B P=\sum_{i=0}^{P-1} s\left(g_{i}-m\right) 2^{i}+s\left(g_{c}-m\right) 2^{P} \quad s(x)= \begin{cases}1 & x>0 \\ 0 & x \leq 0\end{cases}
$$

em que, $m$ é o valor médio das intensidades de nível de cinza da vizinhança de 3 x 3 pixels e é tomado como o novo limiar.

Embora os padrões extraídos pelo LBP tenham grande poder discriminativo, ele perde a estrutura sob algumas circunstâncias. Os autores afirmam que se deve considerar o pixel central a fim de se obter todas as representações dos micropadrões. Na maioria dos casos, o pixel central fornece mais informações do que a sua vizinhança, e assim, deve assumir o maior peso. O valor do pixel que antes era de [0 a 255] passa a ser mapeado de [0 a 510] com informação de textura e forma. De maneira semelhante, é possível mapear o valor de pixel para o intervalo [0 a 30] usando a configuração $\mathrm{LBP}_{4,1}$ (redução do vetor de características).

$\mathrm{Na}$ Figura 2.2, são mostradas as diferenças entre o $\mathrm{LBP}_{4,1}$ original e o $\operatorname{ILBP}_{4,1}$. A Figura 2.2a representa uma vizinhança $3 \times 3$ da imagem que caracteriza uma microestrutura de canto, a Figura 2.2b exibe o resultado do $L B P$ original $^{1}$ e a Figura $2.2 \mathrm{c}$ exibe o resultado do $\operatorname{ILBP}_{4,1}$.

Figura 2.2 Comparação entre $\operatorname{LBP}_{(4,1)}$ e $\operatorname{ILBP}_{(4,1)}$.

\begin{tabular}{|c|c|c|}
\hline$X$ & 1 & $X$ \\
\hline 1 & 20 & 20 \\
\hline$X$ & 20 & $X$ \\
\hline
\end{tabular}

(a) Vizinhança original

\begin{tabular}{|c|c|c|}
\hline$X$ & 0 & $X$ \\
\hline 0 & $*$ & 0 \\
\hline$X$ & 0 & $X$ \\
\hline
\end{tabular}

(b) $L B P_{4,1}$

\begin{tabular}{|c|c|c|}
\hline$X$ & 0 & $X$ \\
\hline 0 & 1 & 1 \\
\hline$X$ & 1 & $X$ \\
\hline
\end{tabular}

(c) $I L B P_{4,1}$

Fonte: Jin et al. (2004).

Aparentemente, não é possível descrever a estrutura de canto corretamente ao usar o LBP original, porque nenhum pixel da vizinhança é maior do que o pixel central. Já com o ILBP definido pela Equação 2.6, o novo limiar é definido, para este exemplo, como 12 $[(1+1+20+20+20) / 5]$ e assim é possível observar que a estrutura local é obtida.

\footnotetext{
${ }^{1}$ Neste trabalho os autores adotam que pixels da vizinhança com intensidades menores ou iguais ao pixel central assumem o valor zero.
} 
Em analogia à extensão do LBP para ILBP, em que o valor médio de vizinhança é usado como o limiar local e o pixel central é incluso no código gerado, o LTP pode ser estendido também para Improved Local Ternary Pattern (ILTP), isto foi proposto pelos autores Nanni, Brahnam e Lumini (2010). Neste descritor é empregado o mesmo esquema de utilização de dois códigos binários concatenados para geração do vetor de características como é realizado no LTP.

\subsection{Center-Symmetric Local Binary Pattern (CS-LBP)}

Heikkila, Pietikainen e Schmid (2006) propuseram o Center-Symmetric Local Binary Pattern (CS-LBP). Neste descritor ao invés de comparar o valor da intensidade de um pixel central com os pixels da vizinhança, o método compara pares simétricos ao centro dos pixels tal como ilustrado na Figura 2.3. Concretamente, cada pixel vizinho é comparado com o seu equivalente de centro simétrico (de um total de quatro comparações ao longo das direções vertical, horizontal, diagonal e anti-diagonal). Como resultado, o CS-LBP só produz $2^{4}=16$ padrões binários diferentes, alcançando uma redução significativa da dimensionalidade e ainda preserva o caráter distintivo.

Figura 2.3 Comparativo dos padrões binários do LBP e CS-LBP para uma vizinhança de 8 pixels

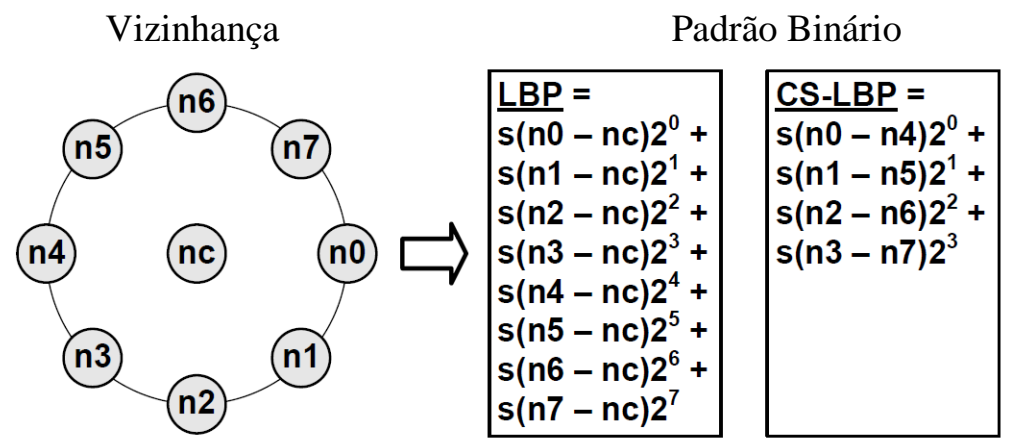

Fonte: Heikkila, Pietikainen e Schmid (2006).

Baseados no CS-LBP, Ferraz, Pereira e Gonzaga (2014) propuseram um novo descritor de região de interesse chamado de Center-Symmetric Local Mapped Pattern (CSLMP) que combina as propriedades desejáveis do CS-LBP utilizando o método LMP, que também encontra-se descrita neste trabalho (Seção 2.8). Esse novo descritor capta as pequenas transições dos pixels na imagem com maior precisão, resultando em um maior número de corretas correspondências do que o CS-LBP. Além disso, não utiliza Principal 
Component Analysis (PCA) para diminuir o tamanho do vetor de características, mas, quantiza o histograma em um número fixo de bins.

\subsection{Completed Local Binary Pattern (CLBP)}

No descritor denominado Completed Local Binary Pattern (CLBP), proposto em (GUO; ZHANG; ZHANG, 2010b) um micropadrão é representado pelo pixel central e pela Local Difference Sign-Magnitude Transform (LDSMT). O pixel central é codificado por um código binário após uma limiarização global, e o mapa binário é denominado CLBP_Center (CLBP_C). Outros dois componentes complementares são obtidos pela LDSMT: os sinais da diferença e as magnitudes da diferença, propondo-se dois operadores, CLBP_Sign (CLBP_S) e CLBP_Magnitude (CLBP_M) para codificá-las.

O método pode ser entendido da seguinte maneira. Dado um pixel central $g_{c}$ e seus $P$ vizinhos $g_{p}, p=0,1, \ldots, P-1$, a diferença entre $g_{c}$ e $g_{p}$ pode ser calculada como $d_{p}=$ $g_{p}-g_{c}$. O vetor de diferença local $\left[d_{0}, \ldots, d_{P-1}\right]$ descreve a estrutura local da imagem em torno de $g_{c}$, a diferença $d_{p}$ pode ser decomposta em duas componentes, usando-se a LDSMT definida pela Equação 2.7,

$$
d_{p}=s_{p} \times m_{p} \quad e \quad\left\{\begin{array}{l}
s_{p}=\operatorname{sign}\left(d_{p}\right) \\
m_{p}=\left|d_{p}\right|
\end{array}\right.
$$

na qual, $\quad s_{p}=\left\{\begin{array}{cc}1 & d_{p} \geq 0 \\ -1 & d_{p}<0\end{array} \quad\right.$ é o sinal de $d_{p}$ e $m_{p}$ é a magnitude de $d_{p}$.

Com a Equação 2.7, o vetor de diferenças local $\left[d_{0}, \ldots, d_{P-1}\right]$ é transformado em um vetor de sinal $\left[s_{0}, \ldots, s_{P-1}\right]$ e um vetor de magnitudes $\left[m_{0}, \ldots, m_{P-1}\right]$, obviamente ambos são complementares e o vetor original de diferenças pode ser reconstruído a partir deles. A Figura 2.4 exibe um exemplo.

Figura 2.4 (a) micropadrão de 3x3 pixels (b) diferenças locais (c) componentes de sinal (d) componentes de magnitude.

\begin{tabular}{|c|c|c|}
\hline 9 & 12 & 34 \\
\hline 10 & $\mathbf{2 5}$ & 28 \\
\hline 99 & 64 & 56 \\
\hline
\end{tabular}

(a)

\begin{tabular}{|c|c|c|}
\hline-16 & -13 & 9 \\
\hline-15 & & 3 \\
\hline 74 & 39 & 31 \\
\hline
\end{tabular}

(b)

\begin{tabular}{|l|l|l|}
\hline-1 & -1 & 1 \\
\hline-1 & & 1 \\
\hline 1 & 1 & 1 \\
\hline
\end{tabular}

(c)

\begin{tabular}{|c|c|c|}
\hline 16 & 13 & 9 \\
\hline 15 & & 3 \\
\hline 74 & 39 & 31 \\
\hline
\end{tabular}

(d)

Fonte: Guo, Zhang e Zhang (2010b). 
A Figura 2.4a exibe um micropadrão de 3 x 3 pixels com o pixel central igual a 25 . O vetor de diferenças (Figura 2.4b) é $[3,9,-13,-16,-15,74,39,31]$. Após aplicar a LDSMT, o vetor dos componentes de sinal (Figura 2.4c) é $[1,1,-1,-1,-1,1,1,1]$ e o vetor das componentes de magnitude é $[3,9,13,16,15,74,39,31]$ (Figura 2.4d).

O descritor CLBP_S é o mesmo que o LBP original definido pela Equação 2.1. Como os componentes de magnitude tem valores contínuos ao invés de valores binários "1" e "-1", eles não podem ser codificados diretamente como os de sinal. Inspirados na estratégia de codificação do CLBP_S e com o intuito de codificar a magnitude em um formato consistente com o do sinal, o CLBP_M é definido pela Equação 2.8,

$$
C L B P_{-} M_{P, R}=\sum_{p=0}^{P-1} t\left(m_{p}, c\right) 2^{p} \quad t(x, c)= \begin{cases}1 & x \geq c \\ 0 & x<c\end{cases}
$$

em que, $c$ é um limiar definido como o valor médio de $m_{p}$ de toda a imagem. Similar ao $\mathrm{LBP}_{\mathrm{P}, \mathrm{R}}^{\mathrm{riu} 2}$, a versão invariante a rotação do CLBP_M $\mathrm{P}_{\mathrm{R}, \mathrm{R}}$ é denominada CLBP_M $\mathrm{M}_{\mathrm{P}, \mathrm{R}}^{\text {riu2 }}$.

Ambos os descritores CLBP_S e CLBP_M produzem códigos binários de modo que possam ser convenientemente utilizados em conjunto para classificação de padrões. Existem duas maneiras para combinar os códigos CLBP_S e CLBP_M: de maneira concatenada ou conjunta. No primeiro modo, calculam-se os histogramas dos códigos do CLBP_S e do CLBP_M separadamente, e, em seguida, são concatenados os dois histogramas. Neste esquema o CLBP pode ser representado como "CLBP_S_M". Na segunda maneira, é calculado um histograma conjunto 2-D dos códigos CLBP_S e CLBP_M. Desta maneira o CLBP é representado como "CLBP_S/M".

O pixel central, que expressa o nível de cinza local da imagem, também tem informações discriminantes. Para torná-lo coerente com o CLBP_S e o CLBP_M, o CLBP_C é definido pela Equação 2.9,

$$
C L B P_{-} C_{P, R}=t\left(g_{p}-\tau_{1}\right)
$$

em que, $t(x)$ é definido como na Equação 2.8 e $\tau_{1}$ é o nível de cinza médio de toda a imagem.

Os três operadores, CLBP_S, CLBP_M, e CLBP_C, podem ser combinados de duas formas, em conjunto ou de maneira híbrida. No primeiro modo, semelhante ao histograma conjunto 2-D, é possível construir um histograma conjunto 3-D deles, representado por "CLBP_S/M/C". No segundo modo, um histograma conjunto 2-D, "CLBP_S/C" ou 
"CLBP_M/C" é construído primeiro, e, em seguida, o histograma é convertido para um histograma 1D, que é então concatenado com o CLBP_M ou o CLBP_S para gerar um histograma conjunto, representado por "CLBP_M_S/C" ou "CLBP_S_M/C".

Um inconveniente destes histogramas híbridos gerados é que podem demandar muito tempo computacional, devido à alta dimensionalidade, dependendo da configuração. Por exemplo, se forem combinados CLBP_S/M/C $8,1+C_{-1}$ CLPS/M/C $16,2+$ CLBP_S/M/ $\mathrm{C}_{24,3}$ obtem-se um vetor de características com dimensionalidade de 2200 bins.

\subsection{Descritores com Transformada de Fourier}

Existem diferentes métodos para tornar o descritor LBP invariante à rotação além da vizinhança circular. Ahonen et al. (2009) aplicam Transformada de Fourier nas ocorrências dos códigos de rotação e o espectro de potência resultante é usado como vetor de características. O descritor é chamado de Local Binary Pattern Histogram Fourier (LBP ${ }^{\mathrm{HF}}$ ). Uma vez que as características de Fourier são calculadas no histograma global de códigos binários na região investigada, o $\mathrm{LBP}^{\mathrm{HF}}$ atinge a invariância de rotação globalmente e, portanto, mantém a distribuição relativa dentro dos grupos de rotação (ZHAO et al., 2012).

No entanto, o descritor $\mathrm{LBP}^{\mathrm{HF}}$ apenas considera códigos binários uniformes (códigos binários com no máximo duas transições entre 0 e 1). Ele foi generalizado em (FERNÁNDEZ et al., 2011) para incluir todos os códigos binários, uniformes e não uniformes, e chamados $\mathrm{LBP}^{\mathrm{DFT}}$. Juntamente com o descritor $\mathrm{LBP}^{\mathrm{DFT}}$, o descritor ILBP $^{\mathrm{DFT}}$ correspondente também foi introduzido em (FERNÁNDEZ et al., 2011).

Diante dos métodos mencionados com a inserção de características de Fourier, Kylberg e Sintorn (2016) propuseram o $\mathrm{LTP}^{\mathrm{DFT}}$ e o $\operatorname{ILTP}^{\mathrm{DFT}}$ que combinam a versão generalizada das características de Fourier de (FERNÁNDEZ et al., 2011) alcançando a invariância global com os descritores LTP e ILTP.

\subsection{Histograms of Oriented Gradients (HOG)}

O descritor denominado de Histograms of Oriented Gradients (HOG) introduzido por Dalal e Triggs (2005) calcula o histograma da orientação dos gradientes na imagem, que resulta em um vetor de características extraídas da imagem. $\mathrm{O}$ algoritmo baseia-se na ideia de que a forma e a aparência de um objeto podem ser descritas pela intensidade dos gradientes ou a direção das bordas, sem um conhecimento prévio da posição de tais bordas. É feita uma 
divisão da imagem em pequenas regiões espaciais chamadas células $(C 1, C 2, \ldots, C n)$ de tamanho 8 x 8 pixels. São calculados os gradientes $G_{X}$ e $G_{Y}$, assim como a magnitude e sua orientação. Para cada célula é calculado um histograma local 1-D das orientações sobre os pixels locais. A Figura 2.5 ilustra este processo.

Figura 2.5 Cálculo do histograma de orientação dos gradientes ${ }^{2}$ de uma célula 8 × 8

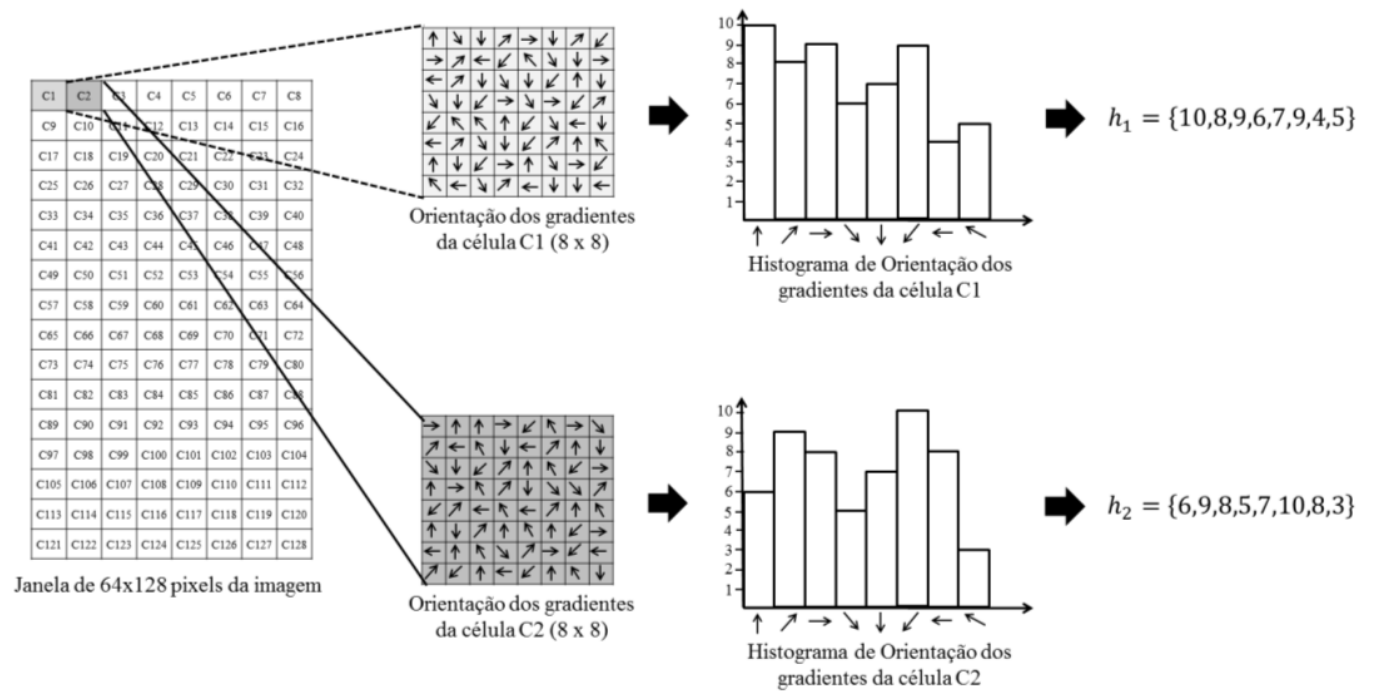

Após os histogramas serem computados, eles são normalizados. A normalização é feita por um acúmulo de histogramas locais em regiões espaciais um pouco maiores chamadas de blocos, com tamanho de 2 x 2 células (Figura 2.6).

Figura 2.6 Acumulado dos histogramas de orientação dos gradientes em blocos para criar a saída F1 do descritor HOG para a janela de 64 x 128 pixels da imagem²

Bloco b1
\begin{tabular}{|l|l|l|l|l|l|l|l|}
\hline h1 & h2 & h3 & h4 & h5 & h6 & h7 & h8 \\
\hline h9 & h10 & h11 & hl1 & h13 & h14 & h15 & h16 \\
\hline h17 & h18 & h19 & h20 & h21 & h22 & h23 & h24 \\
\hline h25 & h26 & h27 & h28 & h29 & h30 & h31 & h32 \\
\hline h33 & h34 & h35 & h36 & h37 & h38 & h39 & h40 \\
\hline h41 & h42 & h43 & h44 & h45 & h46 & h47 & h48 \\
\hline h49 & h50 & h51 & h52 & h53 & h54 & h55 & h56 \\
\hline h57 & h58 & h59 & h60 & h61 & h62 & h63 & h64 \\
\hline h65 & h66 & h67 & h68 & h69 & h70 & h71 & h72 \\
\hline h73 & h74 & h75 & h76 & h77 & h78 & h79 & h80 \\
\hline h81 & h82 & h83 & h84 & h85 & h86 & h87 & h88 \\
\hline h89 & h90 & h91 & h92 & h93 & h94 & h95 & h96 \\
\hline h97 & h98 & h99 & h100 & h101 & h102 & h103 & h104 \\
\hline h105 & h106 & h107 & h108 & h109 & h110 & h111 & h112 \\
\hline h113 & h114 & h115 & h116 & h117 & h118 & h119 & h120 \\
\hline h121 & h122 & h123 & h124 & h125 & h126 & h127 & h128 \\
\hline
\end{tabular}

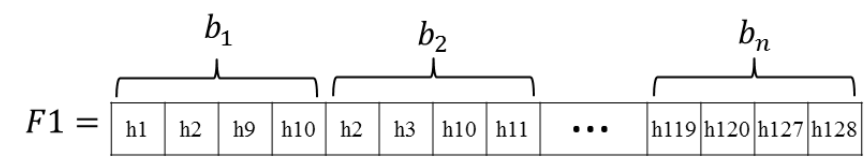

Histogramas acumulados

\footnotetext{
${ }^{2}$ Adaptado da imagem criada pelo ilustrador Juned Munshi, disponível em http://junedmunshiblog.blogspot.com.br/
} 
Os histogramas acumulados são então utilizados para normalizar todas as células naquele bloco. Após a normalização, é coletada uma janela de detecção sobre os histogramas gerados, que consiste na saída do descritor HOG. O descritor HOG utiliza a disposição das orientações dos vetores de gradiente para descrever a forma da imagem analisada. Tal descrição é invariante à posição dos gradientes.

Outros trabalhos também utilizam as informações de orientação para descrição de imagens como o Histogram of Local Orientations (HLO) proposto por Maani, Kalra e Yang (2013) e o Histograms of Second-Order Gradients (HSOG) proposto por HUANG et al. (2014).

\subsection{Local Mapped Pattern (LMP)}

VIEIRA et al. (2012) apresentam um novo descritor baseado em números fuzzy chamado Local Fuzzy Pattern (LFP). Esse método interpreta os valores de nível de cinza de uma vizinhança da imagem como um conjunto fuzzy e cada nível de cinza de um pixel como um número fuzzy. Uma função de pertinência é usada para descrever o grau de pertinência do pixel central a uma determinada vizinhança.

O conceito do LFP evoluiu para um descritor similar que permite o uso de qualquer função e não apenas funções significativas da Lógica Fuzzy. O novo modelo é chamado de Local Mapped Pattern (LMP) (FERRAZ; PEREIRA; GONZAGA, 2014). Este modelo considera a soma das diferenças de cada nível de cinza de uma determinada vizinhança em torno do pixel central como um padrão local que pode ser mapeado para um histograma usando uma função de mapeamento. A Figura 2.7 mostra um exemplo de uma vizinhança de 3 x 3 pixels e um gráfico 3D do respectivo padrão local.

Figura 2.7 Padrão Local das diferenças dos níveis de cinza.

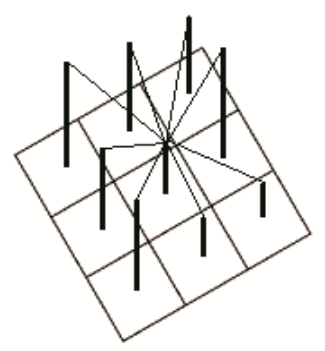

\begin{tabular}{|l|l|l|}
\hline 58 & 50 & 44 \\
\hline 45 & 30 & 62 \\
\hline 50 & 21 & 18 \\
\hline
\end{tabular}

Fonte: Ferraz, Pereira e Gonzaga (2014) 
Cada padrão definido por uma vizinhança de $W \times W$ pixels é mapeado para um bin do histograma $h_{b}$ usando a Equação 2.10,

$$
h_{b}=\operatorname{round}\left[\frac{\sum_{k=1}^{W} \sum_{l=1}^{W}\left(f_{g(i, j)} P(k, l)\right)}{\sum_{k=1}^{W} \sum_{l=1}^{W} P(k, l)} \times(B-1)\right]
$$

em que, $f_{g(i, j)}$ é a função de mapeamento, $P(k, l)$ é uma matriz de pesos com valores prédefinidos para cada posição de pixel dentro da vizinhança e $B$ é o número de bins do histograma. Esta equação representa a soma ponderada de cada diferença de nível de cinza de cada pixel da vizinhança em relação ao pixel central, mapeada para o intervalo $[0,1]$ por uma função de mapeamento, arredondando-se os valores para os $B$ possíveis bins.

A Figura 2.8 ilustra o cálculo do código $L M P$ para um determinado micropadrão de uma imagem de textura.

Figura 2.8 Cálculo do código LMP

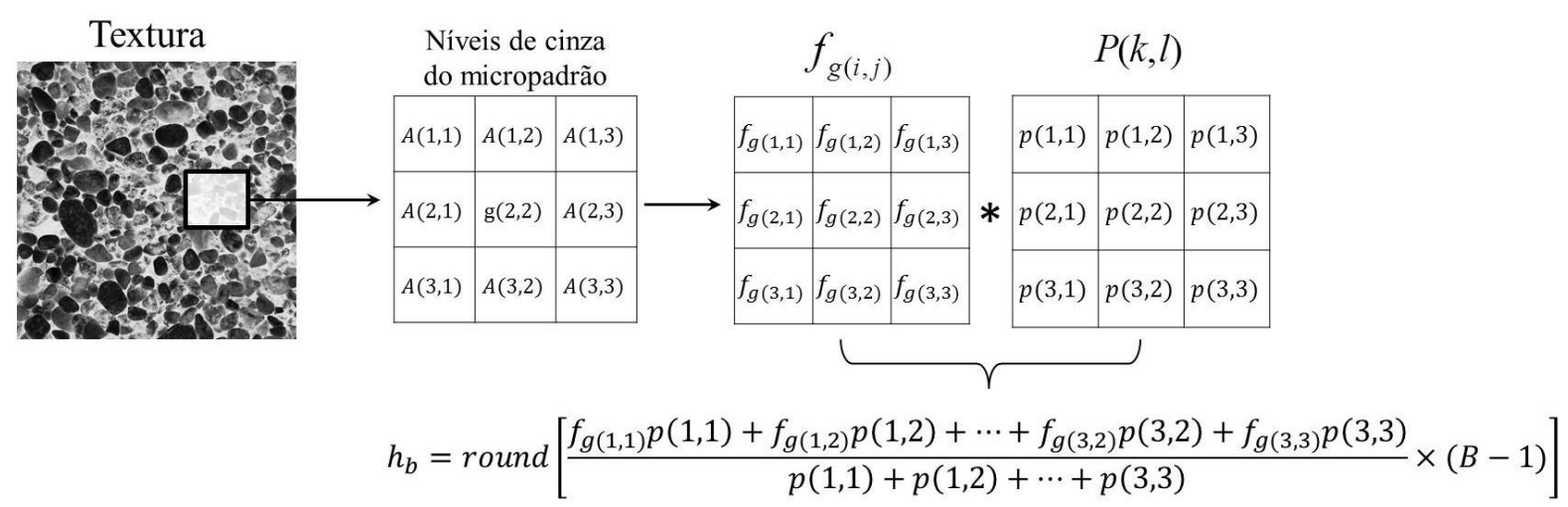

Dependendo da função de mapeamento e da matriz de pesos aplicadas à Equação 2.10 é possível gerar os códigos de algumas abordagens tradicionais previamente publicadas para análise de micropadrões. O LBP básico, por exemplo, pode ser derivado da Equação 2.10 usando-se a função degrau de Heaviside, Equação 2.11,

$$
f_{g(i, j)}=H[A(k, l)-g(i, j)] \text { e } H[A(k, l)-g(i, j)]= \begin{cases}0 & \text { se } A(k, l)-g(i, j)<0 \\ 1 & \text { se } A(k, l)-g(i, j) \geq 0\end{cases}
$$

em que, $g(i, j)$ é o valor do pixel central e $A(k, l)$ os valores dos pixels em uma vizinhança $(k, l)$. 
Levando-se em consideração o LBP básico com uma vizinhança de 3 x 3 pixels, a matriz de pesos será:

$$
P(k, l)=\left[\begin{array}{ccc}
1 & 2 & 4 \\
128 & 0 & 8 \\
64 & 32 & 16
\end{array}\right]
$$

\subsection{Sampled Local Mapped Pattern (S-LMP)}

As características calculadas em uma vizinhança de $3 \times 3$ pixels muitas vezes não podem capturar estruturas de grande escala que podem ser as características dominantes de algumas texturas. Assim, o LMP também pode ser estendido para vizinhanças circulares que consideram a vizinhança de um pixel central como um conjunto de valores dentro de um raio de simetria circular $R$. A configuração da análise multiresolução é dada pelo número de vizinhos, o número de amostras $(P)$ e o valor do raio da vizinhança $(R)$ (CHIERICI, 2015).

Sendo $P$ o número de pixels vizinhos, $g_{p}$ corresponde ao valor de nível de cinza de cada pixel $P$ amostrado, igualmente espaçados em um círculo de raio $R$, formando um conjunto de vizinhos de simetria circular no sentido anti-horário, como ilustrado na Figura 2.9 .

Figura 2.9 Vizinhos de simetria circular

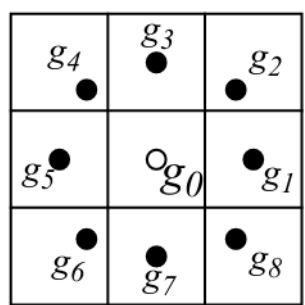

O pixel central $g_{c}$ é localizado nas coordenadas $g_{0}=(0,0)$, e as coordenadas de cada pixel $g_{p}$ são dadas pela Equação 2.12,

$$
g_{p}(x, y)=g_{p}\left(-R \cdot \sin \left(\frac{2 \pi p}{P}\right), R \cdot \cos \left(\frac{2 \pi p}{P}\right)\right)
$$

Os valores de nível de cinza que não estão localizados no centro exato de cada pixel são estimados por meio de interpolação bilinear. Cada padrão definido pelas $P$ amostras é mapeado para um bin do histograma $h_{b}$ usando a Equação 2.13, 


$$
h_{b}=\operatorname{round}\left[\frac{\sum_{p=1}^{P} f_{g(p)}}{P} \times\left(B_{1}-1\right)\right]
$$

em que, $B_{1}$ é o número de bins e $f_{g(p)}$ é a função de mapeamento sigmoidal dada pela Equação 2.14,

$$
f_{g(p)}=\frac{1}{1+e^{\frac{-\left[g(p)-g_{c}\right]}{\beta_{s}}}}
$$

sendo $\beta_{S}$ a inclinação da curva.

Este descritor não utiliza matriz de pesos, conforme proposto na abordagem do LMP original, mas apenas o número de amostras de pixel $(P)$. Isto garante a característica de rotação invariável, não considerando os posicionamentos dos pixels.

\subsection{Considerações Finais}

Neste capítulo discorreu-se sobre diferentes descritores de textura encontrados na literatura. Deu-se especial destaque as técnicas mais aplicadas à classificação de imagens rotacionadas devido ao fato deste trabalho objetivar a otimização do descritor LMP para se enquadrar dentro deste método de análise de textura. No próximo capítulo é descrito o método utilizado para escolha de parâmetros ótimos para descritores baseados no método LMP e no capítulo seguinte os conceitos utilizados nos novos descritores de micropadrões propostos nesta tese. 


\section{Capítulo 3}

\section{Otimização por enxame de partículas}

\subsection{Considerações iniciais}

O comportamento social de alguns grupos de animais, como a organização de abelhas, formigas e pássaros na busca de alimentos ou novos lugares para estabelecer sua nova moradia, tem ganhado destaque em vários estudos. Este último grupo, em especial, despertou um grande interesse de alguns pesquisadores. $\mathrm{O}$ primeiro trabalho computacional nesta área de pesquisa foi um artigo sobre a movimentação de bandos de aves destinada a simular bandos reais para aplicação em filmes e design gráfico, publicado por Craig Reynolds (1987).

Mais tarde, após diversas observações sobre o comportamento de bando de pássaros em revoada, Heppner e Grenander (1990) decidiram modelar esta inteligência coletiva para usá-la em métodos de busca para solução de problemas. Os estudos de Heppner consideravam que o comportamento de várias espécies de pássaros, em bando ao longo do voo, fazia o uso de alguma lógica e de alguma forma de comunicação. Após vários estudos e observações, Heppner descreveu o raciocínio por trás daquele comportamento, qualificando-o como comportamento social.

Em meados dos anos 90, o biólogo Jesper Hoffmeyer (HOFFMEYER, 1994) concebeu uma das melhores definições de enxames em termos de algoritmo: um conjunto de agentes móveis que apresenta a capacidade de se comunicar uns com os outros, direta ou indiretamente no ambiente e convergem coletivamente para a solução de um dado problema.

James Kennedy e Russel Eberhart (1995) inspirados no comportamento social dos pássaros em busca de alimento em uma determinada região estudados por Heppner, desenvolveram o algoritmo de otimização Particle Swarm Optimization (PSO), traduzido para o português como Otimização por Enxame de Partículas. Esta denominação se deu, pois se notou que o modelo escrito por Heppner demonstrava características de um enxame inteligente. Não só o nome do algoritmo, como os demais aspectos do modelo estudado por Heppner ganharam uma nova conotação. A busca pelo alimento ou pelo ninho e a interação entre os pássaros ao longo do voo foram modelados como um mecanismo de otimização. 
Desde então, foram desenvolvidos vários trabalhos sobre o assunto, com aplicações em diversas áreas como, processamento de imagens (SHAIKH; DOYE, 2013; ABDULLAHI; IDRIS; MOHAMMED, 2016; MANDAL; CHATTERJEE; MAITRA, 2017), sistemas de energia elétrica (TZE KIN TEO et al., 2016; PRIBADI et al., 2016 ), telecomunicações (XU; GAO, 2008), entre outros.

\subsection{Inteligência coletiva}

A inteligência coletiva, também comumente referenciada como Inteligência de Colônias ou Inteligência de Enxames, é um conjunto de técnicas baseadas no comportamento coletivo de sistemas auto organizados, distribuídos, autônomos, flexíveis e dinâmicos. Estes sistemas são formados por uma população de agentes computacionais simples que possuem a capacidade de perceber e modificar o seu ambiente de maneira local. Essa capacidade torna possível a comunicação entre os agentes, que captam as mudanças no ambiente geradas pelo comportamento de seus congêneres (SERAPIÃO, 2009).

Embora não exista uma estrutura centralizada de controle que estabeleça como os agentes devem se comportar, e mesmo não havendo um modelo explícito do ambiente, as interações locais geralmente levam ao surgimento de um comportamento global que se aproxima da solução do problema (SERAPIÃO, 2009).

As propriedades principais de um sistema de inteligência de enxame são (MILLONAS, 1994):

- proximidade: os agentes devem ter a noção do espaço e tempo durante seu deslocamento de modo que mudanças possam ser realizadas sem interferir na movimentação de seus vizinhos;

- qualidade: os agentes devem ser capazes de avaliar seus comportamentos, a depender do objetivo almejado, a população deve buscar um caminho em que resulta na melhor solução, garantindo qualidade para seu bando;

- diversidade: capacidade de o sistema reagir a situações inesperadas, prevenindo que os agentes fiquem presos em um local em que não se encontra a melhor solução (mínimo local);

- estabilidade: os agentes do enxame não devem mudar o seu comportamento a cada mudança do ambiente, sendo capaz de avaliar se tal mudança é necessária a fim de praticar um esforço conjunto de todo o bando; 
- adaptabilidade: capacidade de avaliar se as variações ambientais são válidas para mudança no comportamento do bando.

É importante a existência dessas características para garantir o equilíbrio e a estabilidade do bando durante a evolução do processo, classificando-o, portanto, como inteligente.

\subsection{Algoritmo PSO}

O PSO é uma técnica que foi desenvolvida segundo algumas definições de seus autores. Entre elas destacam-se:

- $\quad$ partícula - representa uma possível solução para o problema de otimização;

- população - conjunto de partículas com as mesmas funções objetivo;

- otimizar - encontrar uma situação ótima, ou a melhor situação possível, em um determinado problema;

- $\quad$ BBest - são as melhores soluções ou posições encontradas pelas respectivas partículas até o momento;

- $g B e s t$ - é a melhor solução ou posição encontrada pela população até o momento;

- função fitness ou função objetivo - função de avaliação;

- cognição - cada partícula possui algum conhecimento de sua história (desempenho) e emprega este conhecimento para direcionar seus próximos movimentos no espaço.

O algoritmo básico de otimização por enxame de partículas é inicializado com um grupo de partículas com os valores dos vetores de velocidade e posição gerados aleatoriamente. Em cada iteração, cada partícula $i$ se move em uma determinada direção e é feita uma avaliação de desempenho por uma função objetivo, também chamada de função de aptidão (fitness), que corresponde ao quão ótimo é a solução do problema. Com as partículas do enxame avaliadas, extraem-se o pBest (posição da partícula que levou ao seu melhor desempenho até o momento) e o gBest (melhor desempenho global do sistema até o momento). Em seguida, a partícula atualiza sua velocidade seguindo a Equação 3.1,

$$
v_{i}(t+1)=v_{i}(t)+\varphi_{1} R_{1}\left(p B e s t-x_{i}(t)\right)+\varphi_{2} R_{2}\left(g B e s t-x_{i}(t)\right)
$$


em que:

- $v_{i}(t+1)$ é o novo vetor velocidade da partícula $i$;

- $v_{i}(t)$ é o vetor atual da partícula $i$;

- $\varphi_{1}$ e $\varphi_{2}$ são constantes limitadas a um intervalo finito correspondentes às componentes "cognitiva" e "social" (fatores de aprendizado), respectivamente;

- $\quad R_{1}$ e $R_{2}$ são números aleatórios gerados no intervalo entre 0 e 1 ;

- $\quad p B e s t$ é a melhor solução encontrada pela partícula $i$ até o presente momento;

- gBest é a melhor solução encontrada por todo o enxame até o presente momento, e

- $x_{i}(t)$ é a posição atual da partícula $i$

A equação de atualização da velocidade corresponde à soma de 3 termos distintos: o primeiro refere-se à inércia da partícula; o segundo é um termo cognitivo, relativo à atração da partícula pelo melhor ponto que esta já encontrou. E o terceiro é um termo social, que representa a colaboração entre as partículas.

As constantes $\varphi_{1}$ e $\varphi_{2}$ indicam a importância do caráter social e cognitivo do enxame. Altos valores atribuídos a $\varphi_{1}$ indicam que o algoritmo dará mais importância ao conhecimento individual da partícula. Por outro lado, altos valores atribuídos a $\varphi_{2}$ indicam que o conhecimento global do enxame tem maior importância no algoritmo. Em vários trabalhos tem-se usado valores semelhantes para $\varphi_{1}$ e $\varphi_{2}$ e assim, a velocidade sofrerá influência de forma equivalente tanto do conhecimento individual da partícula como do conhecimento do enxame (SHI, 2014).

Após o cálculo da velocidade da partícula, a sua posição na próxima iteração é estabelecida como uma influência aditiva da posição antiga e da velocidade calculada, sendo expressa pela Equação 3.2.

$$
x_{i}(t+1)=x_{i}(t)+v_{i}(t+1)
$$

Diante das novas posições, caso o critério de parada tenha sido atingido, a solução do problema encontrada é apresentada. Caso contrário, aplica-se novamente a função de avaliação fitness a este enxame, atualizam-se os valores de pBest e gBest (caso seja apresentada uma solução melhor), seguido da atualização da velocidade e posição de cada partícula do enxame. O laço prossegue até o critério de parada ter sido atingido.

Existem duas variantes bastante utilizadas na literatura para a escolha do critério de parada do algoritmo PSO. Uma é pelo número de iterações, ou seja, quando o algoritmo chega 
ao fim, ao atingir a última iteração. A outra é pela função de avaliação (fitness), ou seja, quando o algoritmo chegou ao fim ao alcançar um valor pré-definido para a função.

O pseudocódigo do algoritmo PSO básico, em sua forma original, é dividido em 5 passos:

1. Iniciar a população de $n$ partículas com posições e velocidades aleatórias;

2. Para cada partícula:

a. Avaliar sua aptidão de acordo com a função de avaliação fitness;

b. Calcular a melhor posição da partícula $i$ até o momento ( $p B e s t)$;

3. Descobrir a partícula com a melhor aptidão da população (gBest);

4. Para cada partícula:

a. Atualizar a velocidade da partícula de acordo com a Equação 3.1;

b. Atualizar a posição da partícula de acordo com a Equação 3.2;

5. Se o critério de parada não foi alcançado, retornar ao passo 2 .

\subsection{Parâmetros de controle}

O algoritmo PSO depende de parâmetros que devem ser ajustados de acordo com os problemas as serem otimizados. Os parâmetros a serem ajustados e seus valores típicos são:

1. Número de partículas: o alcance típico é de 20-50, este valor é determinado empiricamente. Um número maior de partículas requer menos iterações para encontrar a solução do problema. Porém, quanto maior a população, mais uso da função de avaliação é necessário, elevando assim, o custo computacional (POLI; KENNEDY; BLACKWELL, 2007).

2. Dimensão das partículas: é determinado pelo problema a ser otimizado.

3. $V_{\text {máx }}$ : responsável pela determinação da variação máxima que uma partícula pode tomar durante uma iteração

4. Fatores de aprendizado $\left(\varphi_{1}\right.$ e $\left.\varphi_{2}\right)$ : geralmente são iguais, é sugerido que sejam adotados $\varphi_{1}=\varphi_{2}=2$, de forma a manter o equilíbrio entre o aprendizado individual de cada partícula e o aprendizado coletivo, no comportamento atual da partícula (SHI; EBERHART, 1998a).

5. Condição de parada: número máximo de iterações que o PSO pode executar e atingir a exigência mínima de erro. 


\subsection{Variações e melhorias dos componentes do PSO}

Com o objetivo de melhorar o desempenho do algoritmo PSO foram propostas por vários autores modificações a partir da formulação original, que visam melhorar a velocidade de convergência sem mudar a estrutura do próprio algoritmo. Isso resulta em otimização local de melhor desempenho e que depende do problema a ser tratado.

\subsubsection{Fator inercial}

Uma das primeiras modificações do algoritmo PSO foi a introdução do fator de inércia em (SHI; EBERHART, 1998b). O fator de inércia é multiplicado pela velocidade anterior na equação da velocidade padrão (Equação 3.1) e diminui linearmente ao longo das iterações. Um peso de inércia diferente de zero introduz uma preferência para a partícula continuar se movendo na mesma direção que ela estava indo na iteração anterior. Diminuindo a inércia ao longo do tempo introduz-se uma mudança na exploração, de busca global para busca local. A junção da equação de atualização de velocidade com o fator de inércia $w(t)$ resulta na Equação 3.3.

$$
v_{i}(t+1)=w(t) * v_{i}(t)+\varphi_{1} R_{1}\left(p B e s t-x_{i}(t)\right)+\varphi_{2} R_{2}\left(g B e s t-x_{i}(t)\right)
$$

Normalmente, $w(t)$, dado pela Equação 3.4, é reduzido linearmente, a partir de $w_{\text {inicial }}$ até $w_{\text {final }}$, em cada iteração. Um bom ponto de partida é definir $w_{\text {inicial }}$ como 0,9 e $w_{\text {final }}$ como 0,4 .

$$
w(t)=\frac{\left(T_{\max }-t\right) x\left(w_{\text {inicial }}-w_{\text {final }}\right)}{T_{\max }}+w_{\text {final }}
$$

em que, $\mathrm{T}_{\max }$ é a quantidade máxima de iterações para busca do valor ótimo.

\subsubsection{Fator de constrição}

Outra melhoria do algoritmo PSO proposta, foi o coeficiente ou fator de constrição introduzindo por Clerc e Kennedy (2002). Este fator propõe um novo método de escolha dos valores de $\varphi_{1} e \varphi_{2}$, equilibrando a necessidade de busca local e global, de forma a assegurar a 
convergência. Foi proposto que estes parâmetros sejam determinados analiticamente por meio do fator de constrição definido pela Equação 3.5.

$$
\chi=\frac{2 k}{\left|2-\varphi-\sqrt{\varphi^{2}-4 \varphi}\right|} \quad \varphi=\varphi_{1}+\varphi_{2}, \varphi>4
$$

Assim, a equação de atualização de velocidade usando este fator fica então definida, pela Equação 3.6.

$$
v_{i}(t+1)=\chi\left[v_{i}(t)+\varphi_{1} R_{1}\left(p B e s t-x_{i}(t)\right)+\varphi_{2} R_{2}\left(g B e s t-x_{i}(t)\right)\right]
$$

Clerc e Kennedy (2002) descobriram que, modificando $\varphi$, as características de convergência do sistema podem ser controladas. Tipicamente, $k=1$ e $\varphi_{1}=\varphi_{2}=2,05$, e $\varphi$ é definido como 4,1 .

\subsection{Considerações finais}

Neste capítulo foi apresentada uma revisão do método de otimização por enxames de partículas. Cabe ressaltar que o algoritmo PSO possui parâmetros intrínsecos a seu funcionamento, que devem ser ajustados a cada problema tratado. Tais parâmetros influenciam diretamente no desempenho do mesmo, especialmente no tratamento de problemas mais complexos. No Capítulo 5 encontram-se definidos os parâmetros aplicados no presente trabalho. 


\section{Capítulo 4}

\section{Descritores Propostos}

\subsection{Considerações iniciais}

Os métodos baseados na abordagem LBP existentes obtêm um padrão binário a partir das diferenças entre o pixel central e os pixels da vizinhança em um micropadrão. A expressão binária tornou o método LBP popular devido à simplificação obtida na relação dos pixels comparados. Mas, por outro lado, esta formulação binária apesar de simples, também pode descartar informações das diferenças locais na presença de ruídos, pois, a menor ou maior variação acima ou abaixo do valor de nível de cinza do pixel central ou de um pixel vizinho pode mudar todo o padrão LBP significativamente.

A Figura 4.1 ilustra dois micropadrões diferentes em uma vizinhança de 3 x 3 pixels com o mesmo valor de código LBP gerado.

Figura 4.1 Diferentes micropadrões com mesmo código $L B P$ gerados

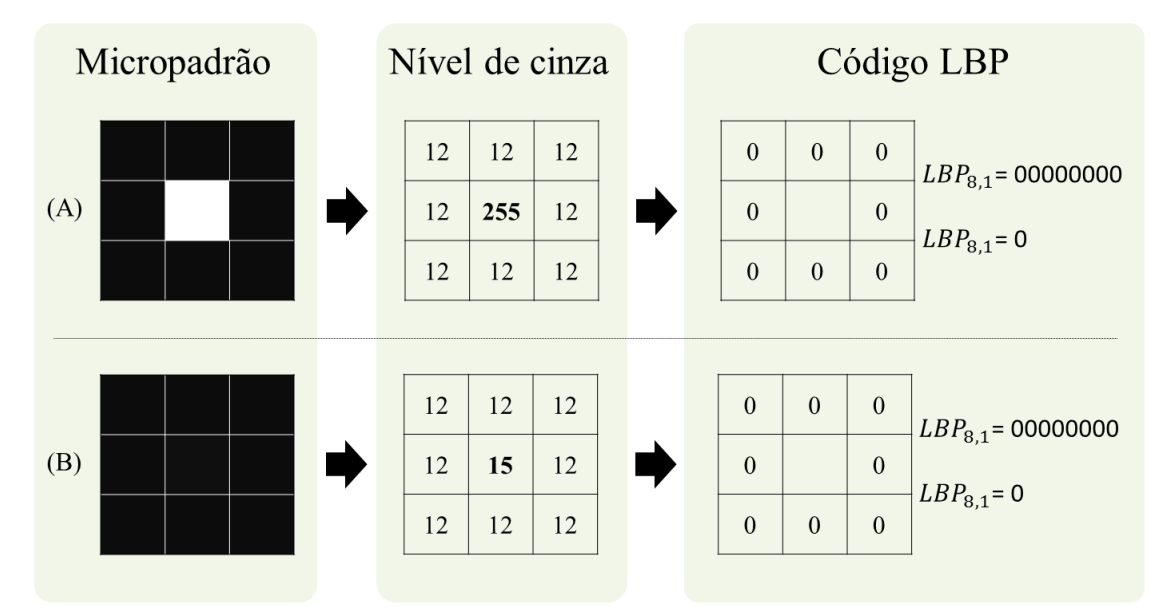

Levando-se em conta a percepção visual humana, o micropadrão representado na imagem A representa uma estrutura pontual, e o micropadrão representado na imagem B 
representa um estrutura plana, mas os códigos gerados de estruturas diferentes tem a mesma codificação e esta representação simplificada provoca redução de informação.

Uma maneira simples de superar essa desvantagem é adotar uma resposta apropriada a cada padrão encontrado de acordo com as suas diferenças locais e isto vem sendo realizado nos descritores baseados no descritor LMP. Os códigos gerados para os micropadrões da Figura 4.1 são diferentes devido ao mapeamento realizado pelo descritor LMP pelo uso da função sigmoide que nada mais é do que uma suavização da função degrau utilizada no descritor LBP. Esta suavização permite a descrição de nuances presentes nos micropadrões que não são consideradas nos descritores LBP. A Figura 4.2 ilustra os códigos gerados pelo LMP básico para as diferentes estruturas apresentadas utilizando a mesma função de pertinência e parâmetros $\beta$ e $B$.

Figura 4.2 Diferentes micropadrões com diferentes códigos LMP gerados.

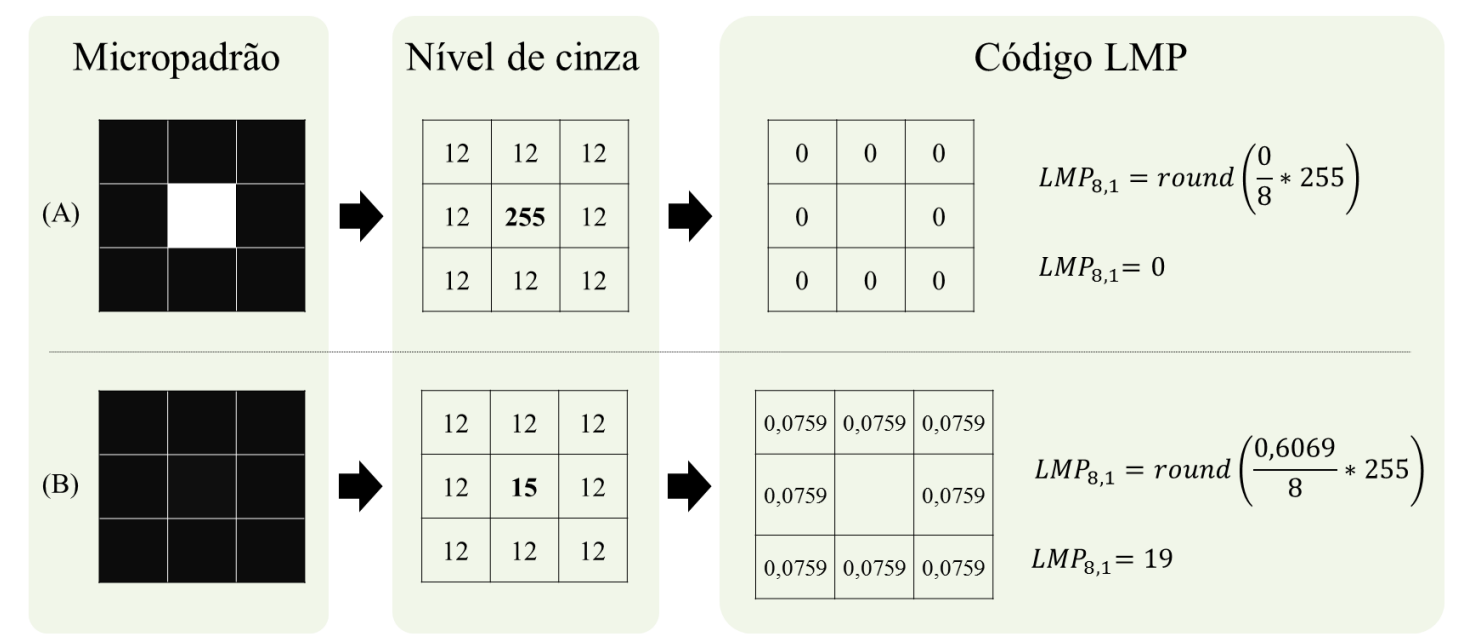

Com a função de mapeamento é possível gerar diferentes códigos para micropadrões distintos e manter informação. Devido ao melhor desempenho do descritor LMP, novos desdobramentos vêm sendo propostos pelo grupo do LAVI a fim de se obter uma melhor descrição em imagens de texturas. No trabalho de Souza, Vieira e Gonzaga (2015) o método LMP é aplicado na análise da textura da íris para reconhecimento biométrico. Ferraz e Gonzaga (2016) utilizam para classificação de objetos. Já Negri et al. (2017) utilizam na classificação de imagens de textura coloridas sob variação de iluminação.

Como continuidade das aplicações do método LMP e diante da necessidade de descritores também robustos à variação de rotação, esta tese propõe dois novos descritores: o Sampled Local Mapped Pattern Magnitude (SLMP_M) e o Completed Local Mapped Pattern 
(CLMP). O que os diferencia dos desdobramentos do método LMP propostos até então, é que ambos os descritores propostos nesta tese utilizam a informação de magnitude da diferença entre os níveis de cinza dos pixels da vizinhança e o pixel central do micropadrão analisado.

Em muitas aplicações, especialmente na inspeção visual industrial, a iluminação pode ser controlada com precisão. Em tal situação, um descritor de textura invariante a mudanças de escalas de cinza pode desperdiçar informações úteis e, ao adicionar informações que dependem da escala de cinza, é possível aumentar a precisão do método (PIETIKÄINEN et al., 2011).

O contraste é uma propriedade da textura normalmente considerada como uma informação muito importante para nosso sistema de visão, porém, tanto o descritor LMP como o descritor LBP, em suas formulações básicas, não levam em conta a magnitude das diferenças de nível de cinza, apenas a informação de sinal. No entanto, o sinal e a magnitude são complementares e podem ser usados para reconstruir exatamente as diferenças de nível de cinza e, vários autores utilizam essa medida na formulação dos seus descritores como, por exemplo, nos trabalhos de Dong et al. (2017) e Pan et al. (2017).

De maneira geral, a textura é distinguida não só pelos padrões de textura, mas também pela intensidade dos padrões. A textura pode assim ser considerada como um fenômeno bidimensional caracterizado por duas propriedades ortogonais: estrutura espacial (padrões) e contraste (a intensidade dos padrões) (MÄENPÄ̈̈, 2003). A Figura 4.3 ilustra os aspectos bidimensionais de um micropadrão de textura em uma vizinhança de 3 x 3 pixels.

Figura 4.3 Representação estrutural e de intensidade de um micropadrão de 3 x 3 pixels.

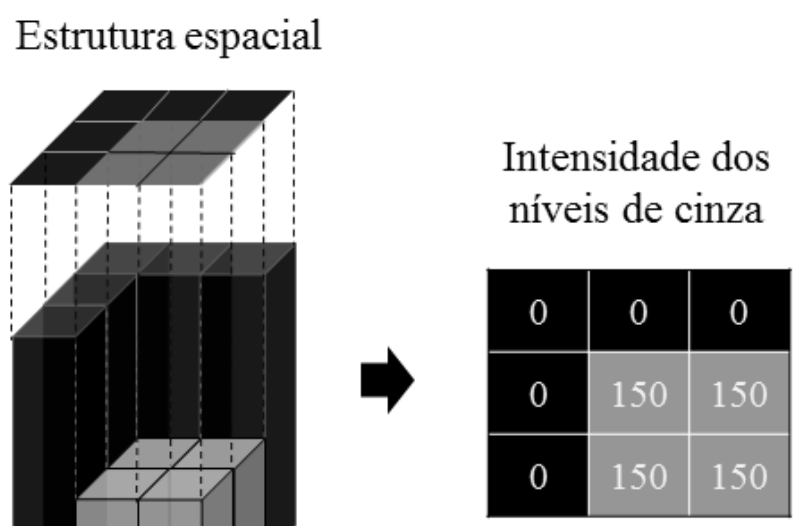


A informação do padrão é independente da escala de cinza, enquanto que o contraste não é. Por outro lado, o contraste não é afetado pela rotação, mas os padrões são. Estas duas medidas complementam-se mutuamente de uma forma muito útil e esta característica é levada em consideração na formulação dos descritores apresentados nas seções seguintes.

\subsection{Completed Local Mapped Pattern (CLMP)}

Motivada pelo notável desempenho de classificação do descritor CLBP que é formado pelas diferentes combinações dos três operadores (CLBP_S, CLBP_M e CLBP_C), é apresentado nesta seção o Completed Local Mapped Pattern (CLMP). Na formulação deste, as diferenças locais da imagem também são decompostas em dois componentes complementares: o sinal e a magnitude que dão origem aos dois operadores CLMP_S e CLMP_M. E, para tirar proveito da informação global da textura da maneira mais simples, é calculada a diferença entre o valor do pixel central e o valor médio da imagem inteira pelo operador CLMP_C.

Considerando a vizinhança de um pixel central como um conjunto de valores igualmente espaçados em um círculo de raio $R$, o número de pixels vizinhos é $P$ e $g_{(p)}$ corresponde ao nível de cinza de cada pixel $p$. O pixel central é localizado nas coordenadas $g_{c}=(0,0)$ e cada pixel $g_{(p)}, \quad p=0,1, \ldots,(P-1)$ tem as coordenadas dadas por $(-R \cdot \operatorname{sen}(2 \pi p / P), R \cdot \cos (2 \pi p / P))$. Os valores dos níveis de cinza dos pixels não localizados exatamente ao centro de cada pixel, são estimados por interpolação bilinear.

O operador CLMP_S é exatamente o mesmo que o SLMP, que computa a informação de sinal de um micropadrão em uma vizinhança específica ao usar a função de mapeamento da Equação 4.1.

$$
f_{C L M P_{-} S(p)}=\frac{1}{1+e^{\frac{-\left(g_{(p)}-g_{c}\right)}{\beta_{S}}}}
$$

na qual, $\beta_{S}$ é a inclinação da curva da função de mapeamento do operador de sinal.

Os códigos CLMP_S são gerados pela Equação 4.2. Para cada operador, os códigos são armazenados em uma matriz individual chamada "mapa de códigos". 


$$
C L M P_{-} S_{(P, R)}=\text { round }\left[\frac{\sum_{p=1}^{P} f_{C L M P} S_{(p)}}{P} \times\left(B_{S}-1\right)\right]
$$

em que, $B_{S}$ é o valor máximo no mapa de código da informação de sinal.

O operador CLMP_M utiliza a função de mapeamento apresentada na Equação 4.3 para extrair informação de magnitude do micropadrão de textura.

$$
f_{C L M P_{-} M(p)}=\frac{1}{1+e^{\frac{-\left(m_{p}-c\right)}{\beta_{m}}}}
$$

sendo, $m_{p}=\left|g_{c}-g_{(p)}\right|$ a magnitude da diferença entre o pixel central $g_{c}$ e os pixels da vizinhança $g_{p}, c$ o valor médio da magnitude calculada na imagem inteira e $\beta_{m}$ a inclinação da curva da função de mapeamento do operador de magnitude.

Os códigos CLMP_M são gerados pela Equação 4.4.

$$
C L M P_{-} M_{(P, R)}=\operatorname{round}\left[\frac{\sum_{p=1}^{P} f_{\left.C L M P_{-} M_{(}\right)}}{P} \times\left(B_{M}-1\right)\right]
$$

em que, $B_{M}$ é o valor máximo da informação de magnitude no mapa de código.

Os parâmetros $\beta_{S}$ e $B_{S}$ (inclinação da curva e o valor máximo no mapa de código da informação de sinal), $\beta_{M}$ e $B_{M}$ (inclinação da curva e o valor máximo no mapa de código da informação de magnitude) usados nos operadores CLMP_S e CLMP_M, respectivamente, podem ser ajustados para a aplicação desejada.

O CLMP_C é definido da mesma maneira que o CLBP_C. O vetor de características é gerado pela Equação 4.5,

$$
C L M P_{-} C_{(P, R)}=t\left(g_{(p)}-\tau_{1}\right) \quad t(x, c)= \begin{cases}1 & x \geq c \\ 0 & x<c\end{cases}
$$

em que, $\tau_{1}$ é o nível de cinza médio de toda a imagem.

Após a geração dos mapas de código de cada operador, o vetores de característica são obtidos a partir da combinação dos operadores em diferentes maneiras. Para comparar o 
desempenho do descritor proposto, foram avaliadas as mesmas combinações usadas pelo descritor CLBP. Os três operadores, CLMP_S, CLMP_M e CLMP_C, podem ser combinados de duas maneiras, conjunta ou híbrida. Na primeira maneira, calcula-se o histograma 2-D conjunto dos códigos CLMP_S e CLMP_M; esta combinação é representada por CLMP_S/M. Semelhante ao histograma conjunto 2-D é possível construir um histograma conjunto 3-D dos três operadores, que dá origem ao descritor CLMP_S/M/C. Na segunda maneira, um histograma conjunto 2-D dos descritores CLMP_S/C ou CLMP_M/C é construído primeiro e, em seguida, o histograma é convertido em um histograma 1-D, que é então concatenado com o histograma do CLMP_M ou CLMP_S para gerar um novo histograma conjunto, que dá origem aos descritores CLMP_M_S/C ou CLMP_S_M/C.

Durante as combinações dos operadores CLMP_S, CLMP_M e CLMP_C, os valores do mapas de códigos são quantizados, de modo que os vetores de características gerados tenham o mesmo tamanho que os obtidos pela combinação dos operadores CLBP. A Tabela 4.1 apresenta o número de elementos dos vetores de característica para diferentes configurações $(\mathrm{P}, \mathrm{R})$ para todos os operadores CLMP. Neste trabalho, optou-se por quantizar os vetores de características para ter o mesmo tamanho do CLBP e realizar uma comparação mais precisa.

Tabela 4. 1 Número de elementos dos vetores de características do CLMP.

\begin{tabular}{l|ccc}
\cline { 2 - 4 } & \multicolumn{4}{c|}{ Configuração $(P, R)$} \\
\cline { 2 - 4 } & $(P=8, R=1)$ & $(P=16, R=2)$ & $(P=24, R=3)$ \\
\hline \multicolumn{1}{c}{ Descritor } & \multicolumn{4}{c|}{ Número de elementos } \\
\hline \hline CLMP_M/C & 20 & 36 & 52 \\
\hline CLMP_S_M/C & 30 & 54 & 78 \\
CLMP_S/M & 100 & 324 & 676 \\
\hline CLMP_S/M/C & 200 & 648 & 1.352 \\
\hline
\end{tabular}

\subsection{Sampled Local Mapped Pattern Magnitude (SLMP_M)}

O descritor chamado Sampled Local Mapped Pattern Magnitude (SLMP_M) (VIEIRA; NEGRI; GONZAGA, 2016) considera a magnitude entre pixels da vizinhança para extrair características invariantes à rotação. Combina o descritor SLMP com informações relacionadas à magnitude entre pixels da vizinhança. $\mathrm{O}$ vetor de características do descritor SLMP_M é construído ao se concatenar o histograma proveniente do descritor SLMP, descrito na seção 2.10, e um histograma de $B_{2}$ bins e $P$ pontos amostrados, com informação 
de magnitude extraída da textura pela Equação 4.6 com função de mapeamento dada pela Equação 4.7,

$$
\begin{gathered}
h_{b}=\operatorname{round}\left[\frac{\sum_{p=1}^{P} f_{g(p)}}{P} \times\left(B_{2}-1\right)\right] \\
f_{g}(p)=\frac{1}{1+e^{\frac{-\left(m_{p}-c\right)}{\beta_{2}}}}
\end{gathered}
$$

em que $m_{p}=\left|g_{c}-g_{p}\right|$ é a magnitude da diferença entre o pixel central $g_{c}$ e os pixels da vizinhança $g_{p}, c$ é o valor médio da magnitude calculada na imagem inteira e $\beta_{2}$ é a inclinação da curva da função de mapeamento.

O SLPM_M pode ser considerado com um caso especial da combinação dos operadores do CLMP, visto que a combinação via concatenação, apenas dos operadores de sinal e magnitude, não foi realizada anteriormente.

\subsection{Considerações Finais}

Neste capítulo foram apresentados os descritores de imagens de textura baseados na análise da informação de sinal e magnitude de um micropadrão. O primeiro denominado CLMP é inspirado no conhecido descritor CLBP e o segundo descritor denominado SLMP_M é um caso especial da combinação dos operadores de sinal e magnitude do CLMP. Conforme justificado, a estrutura dos micropadrões bem como a intensidade dos níveis de cinza são informações complementares e não devem ser descartadas. Assim, a inserção da informação de magnitude na geração do vetor de características mostra-se adequada no sentido de se manter mais informação discriminante em aplicações com imagens de textura. 


\section{Capítulo 5}

\section{Material e Método}

\subsection{Considerações iniciais}

Neste capítulo é apresentada a formulação do método de otimização por enxames de partículas para obtenção de parâmetros ótimos dos descritores propostos, com o objetivo de encontrar um $\beta$ (inclinação da curva sigmoidal) ótimo e reduzir a sua dimensionalidade alterando-se o parâmetro $B$ (número de bins) mantendo a sua capacidade de discriminação. A medida de acurácia é utilizada para quantificar as informações após a otimização dos parâmetros.

Após o processo de otimização de parâmetros, os descritores propostos nesta tese são comparados com cinco descritores presentes na literatura: CLBP, LBPDFT, ILBPDFT, LTPDFT, ILTPDFT. Foram conduzidos cinco experimentos utilizando duas bases de imagens de textura Kylberg Sintorn Rotation Dataset (KYLBERG; SINTORN, 2015) e Brodatz Texture Rotation Dataset $^{3}$. Esta desenvolvida pela autora no Laboratório de Visão Computacional (LAVI) a partir das imagens de textura do Álbum de Brodatz cujo processo de aquisição também é descrito neste capítulo.

\subsection{Otimização dos parâmetros $\beta$ e $B$ dos descritores baseados no método LMP}

O descritor desenvolvido no LAVI, denominado de LFP (proposto na dissertação de Mestrado da autora (VIEIRA, 2013) e sua evolução denominada de LMP, aplicados à classificação de texturas mostraram-se bastante eficientes nas mais diversas aplicações. Porém, o LMP apresenta as dificuldades inerentes aos métodos paramétricos: definir os parâmetros que atendam adequadamente à aplicação. Desta maneira, este trabalho propõe o uso do método de otimização por enxame de partículas para que se possam atingir os

\footnotetext{
${ }^{3}$ http://imagem.sel.eesc.usp.br/base
} 
parâmetros que proporcionam as melhores taxas de acurácia ao se aplicar os descritores baseados no método LMP nos processos de classificação de imagens de textura.

A Equação 5.1 que gera um histograma de $B$ bins no método LMP para vizinhanças circulares de $P$ pontos amostrados, permite o uso de diferentes funções de mapeamento para a descrição de uma imagem.

$$
h_{b}=\operatorname{round}\left[\frac{\sum_{p=1}^{P} f_{g(p)}}{P} \times(B-1)\right]
$$

Em todos os descritores propostos a curva sigmoidal definida pela equação geral 5.2 é usada como função de mapeamento,

$$
f_{g}=\frac{1}{1+e^{\frac{-\left(g_{(p)}-g_{c}\right)}{\beta}}}
$$

em que, $\beta$ é o parâmetro que define a inclinação da curva.

Além do parâmetro $\beta$, o número de bins do histograma $(B)$ é outro parâmetro que pode ser otimizado para um melhor desempenho do método, acarretando também na redução de dimensionalidade do vetor de características gerado, diminuindo assim, o custo computacional no seu processamento.

Até o presente momento, os trabalhos que utilizam o método LMP, definem os valores dos parâmetros $\beta$ e $B$ empiricamente e separadamente, isto demanda tempo e muitas vezes não leva a uma escolha de parâmetros adequada para o problema. Dessa maneira, o uso de otimização por enxame de partículas (PSO) mostra-se uma excelente ferramenta para encontrar combinações beta-bin que possam gerar resultados ótimos.

As propostas de modificação na equação de velocidade detalhadas na seção 3.6 visam o balanceamento da habilidade de exploração de amplitude e profundidade, que têm em vista melhorar a velocidade de convergência e a qualidade da solução encontrada. Neste trabalho, utilizou-se o algoritmo PSO básico que se mostrou eficiente na busca de parâmetros ótimos.

A Figura 5.1 ilustra um diagrama de fluxo que representa a integração entre o algoritmo de otimização por enxame de partículas e a função de mapeamento da equação que define o método LMP. 
Figura 5.1 Diagrama de fluxo do algoritmo PSO para ajuste dos parâmetros do LMP.

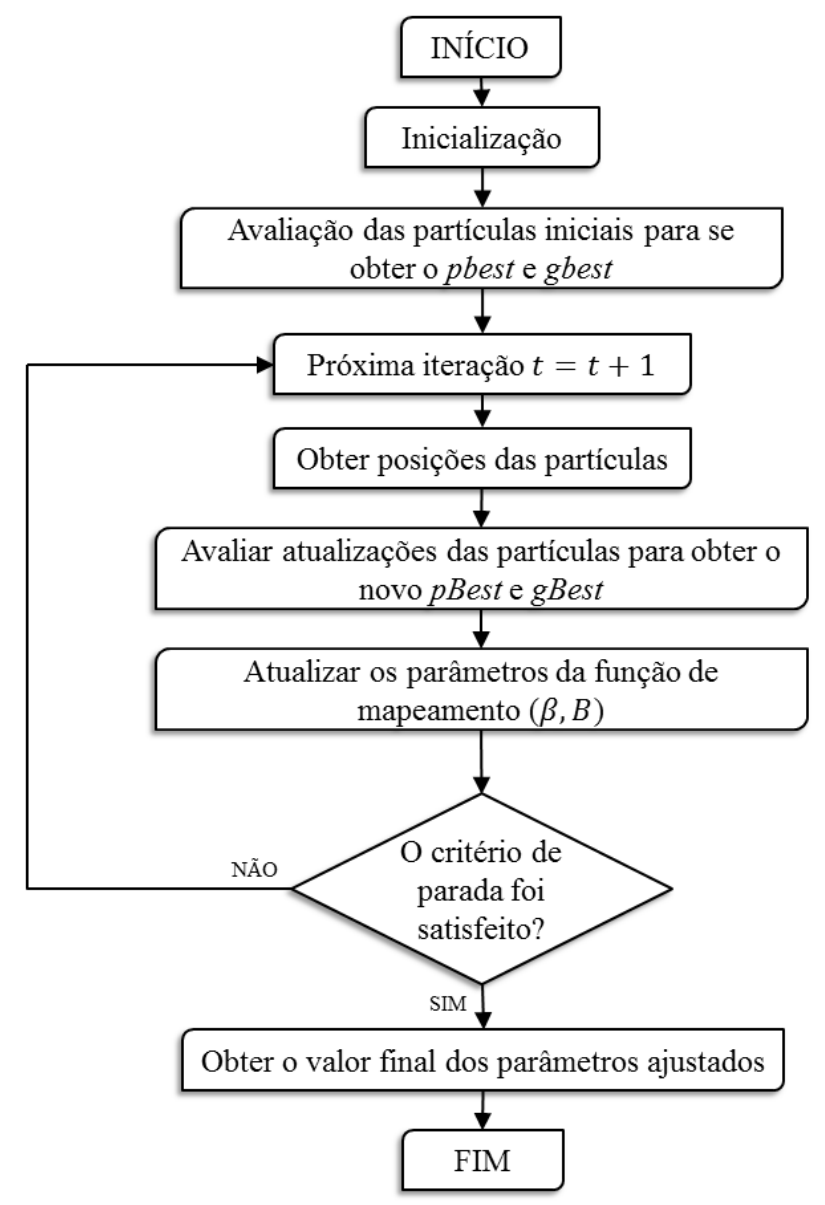

Sabendo-se que a posição de uma partícula é dada por uma função $f(x(i), y(i))$, a função fitness escolhida para se avaliar o comportamento das partículas é dada pela Equação 5.3 .

$$
\text { fitness }(x(i), y(i))=(x(i)-50)^{2}+(y(i)-50)^{2}
$$

A Tabela 5.1 exibe os parâmetros de configuração escolhidos empiricamente para o algoritmo PSO para otimização dos parâmetros dos descritores propostos.

Tabela 5. 1 Parâmetros de configuração do PSO

\begin{tabular}{cc}
\hline Parâmetro & Valor \\
\hline \hline Quantidade de partículas & 5 \\
\hline Quantidade de iterações & 300 \\
\hline$\varphi_{1}$ & 0,5 \\
\hline$\varphi_{2}$ & 0,5 \\
\hline
\end{tabular}


Dois critérios de parada foram adotados, o erro que é calculado pela distância entre as partículas e a solução ótima. Quando uma partícula alcança uma posição muito próxima da solução ótima (erro $\leq 0,01$ ), o algoritmo para de executar imediatamente. O segundo critério é o número de iterações $\leq 300$; esta condição de parada foi adicionada, pois nem sempre é possível encontrar a solução ótima em um tempo curto.

\subsection{Kylberg Sintorn Rotation Dataset}

Para avaliar o desempenho de diferentes descritores invariantes à rotação, Kylberg e Sintorn (KYLBERG; SINTORN, 2015) apresentaram uma base de imagens de texturas capturadas fisicamente em diversas rotações previamente estabelecidas (hardware) e de texturas rotacionadas computacionalmente por meio de diferentes métodos de interpolação (software). Esta base é composta de 25 classes de texturas naturais. A Figura 5.2 exibe uma imagem de cada classe. As imagens originais tem o tamanho de 2592 x 1728 pixels.

Figura 5.2 Amostras das 25 classes da Kylberg Sintorn Rotation Dataset.
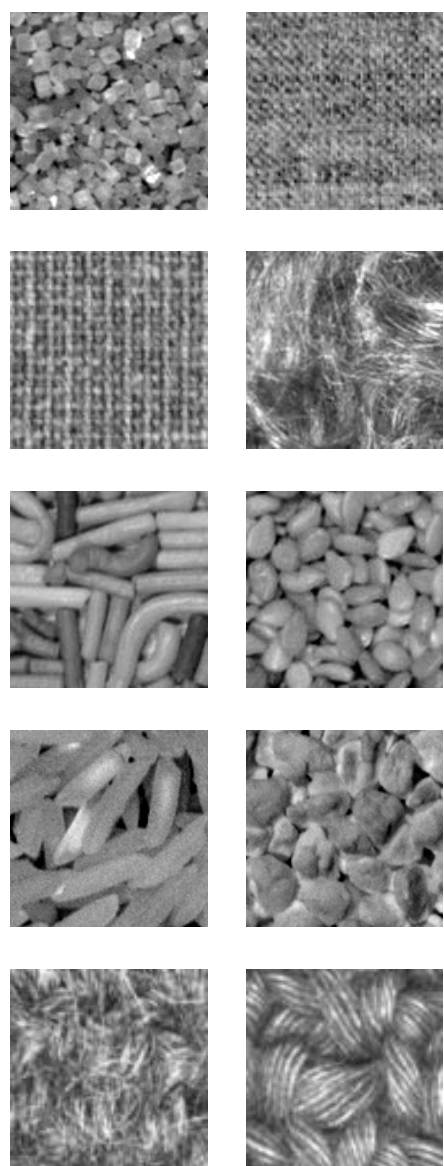
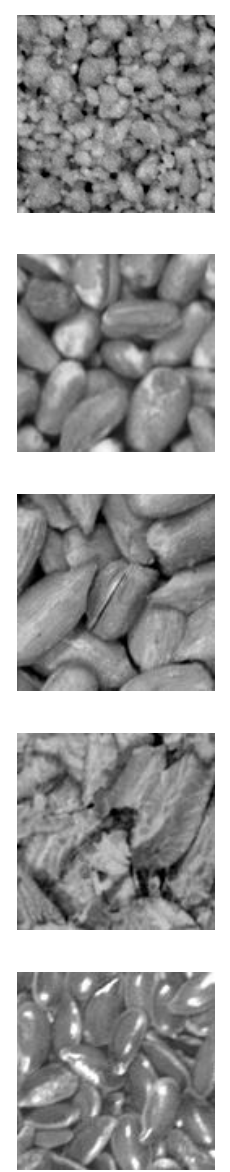
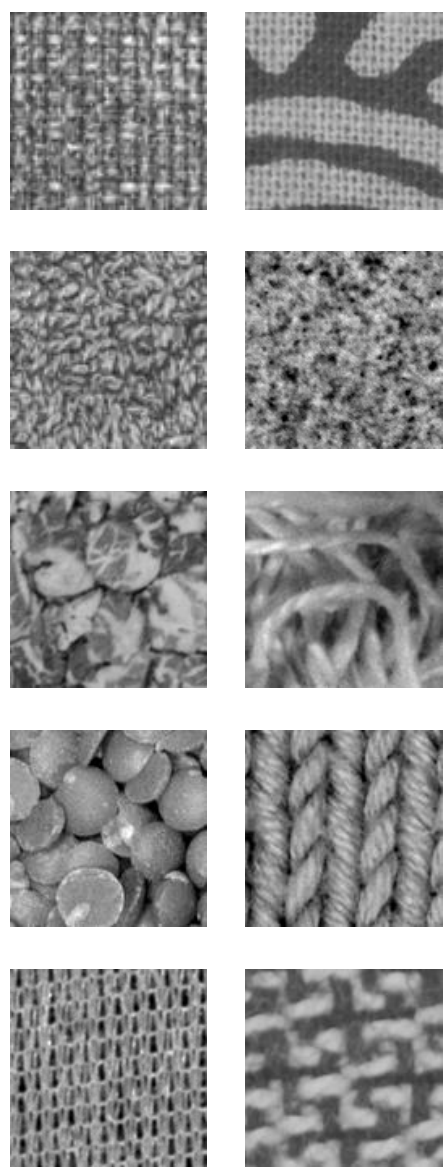
Todas as imagens capturadas são divididas em 100 amostras de texturas menores com tamanho 122 x 122 pixels. Para avaliar diferentes tamanhos de amostras também foram extraídas amostras de tamanho 90 x 90 pixels e 70 x 70 pixels.

\subsubsection{Rotação por Hardware (HW)}

O procedimento de aquisição das imagens foi configurado de maneira que a câmera fosse rotacionada em torno do eixo central da sua lente. Ao rotacionar a câmara em vez da textura, é possível manter as mesmas condições de iluminação ao longo da aquisição de imagem. Para cada classe de textura, uma imagem foi adquirida em cada orientação. As texturas são rotacionadas em nove orientações $\theta \in\left\{0^{\circ}, 40^{\circ}, 80^{\circ}, 120^{\circ}, 160^{\circ}, 200^{\circ}, 240^{\circ}, 280^{\circ}\right.$ e $320^{\circ}$ \} eliminando-se múltiplos de $90^{\circ}$, ângulos para os quais a escolha de métodos de interpolação não iria fazer diferença.

\subsubsection{Rotação por Software}

Nesta base foram utilizados cinco métodos de interpolação computacional: Nearest Neighbor, Linear, Cúbica de terceira ordem, cúbica B-spline e Lanczos. As imagens de texturas com ângulo de orientação $0^{\circ}$ foram usadas para interpolar as outras oito orientações usando cada uma das cinco abordagens de interpolação.

\section{4 Álbum de Brodatz Rotacionado}

Para validar os descritores propostos nesta tese em mais de uma base, a autora optou por desenvolver uma nova base rotacionada a partir do Álbum de Brodatz (BRODATZ, 1966) no Laboratório de Visão Computacional. Levando em conta que as bases existentes na literatura apresentam no máximo 25 classes de texturas e 10 ângulos de rotação, sentiu-se a necessidade de mais uma base com maior diversidade de ângulos de rotação, maior quantidade de classes e também diferentes tamanhos de amostras. Assim, como na base Kylberg Sintorn, as imagens foram rotacionadas por hardware e também por software utilizando-se o mesmo procedimento para processamento das imagens. 


\subsubsection{Rotação por hardware}

Para o processo de captura e digitalização das imagens foram utilizados os seguintes dispositivos e equipamentos:

- Câmera fotográfica Sony DSC-W350, 14.1 megapixels, sensor super HAD CCD tipo 1/2.3 (7,76 mm), lentes Carl Zeiss Vario-Tessar;

- Tripé universal Sony e mesa de apoio;

- Transferidor com eixo giratório $360^{\circ}$ (Figura 5.3).

Figura 5.3 Transferidor com eixo giratório.

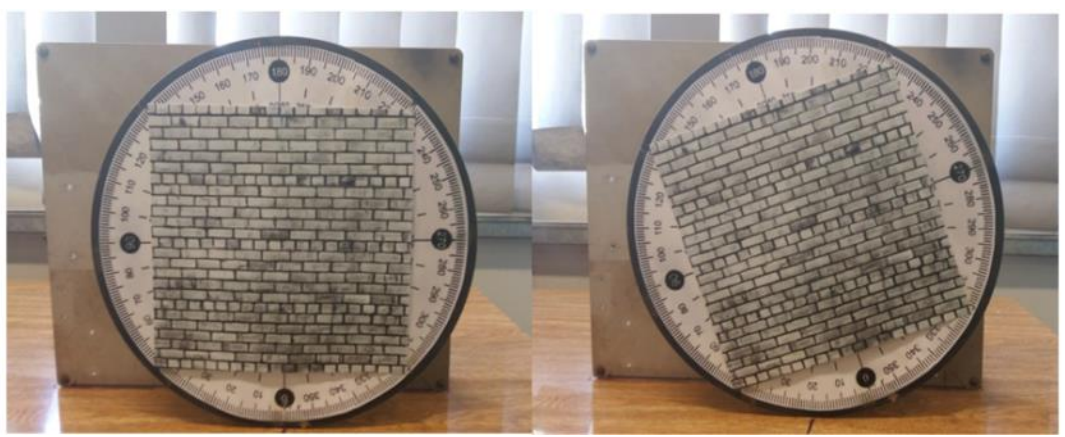

A distância estabelecida entre a lente da câmera fotográfica e a imagem foi de $16,5 \mathrm{~cm}$ (Figura 5.4).

Figura 5.4 Ilustração da distância entre a lente da câmera e a textura.

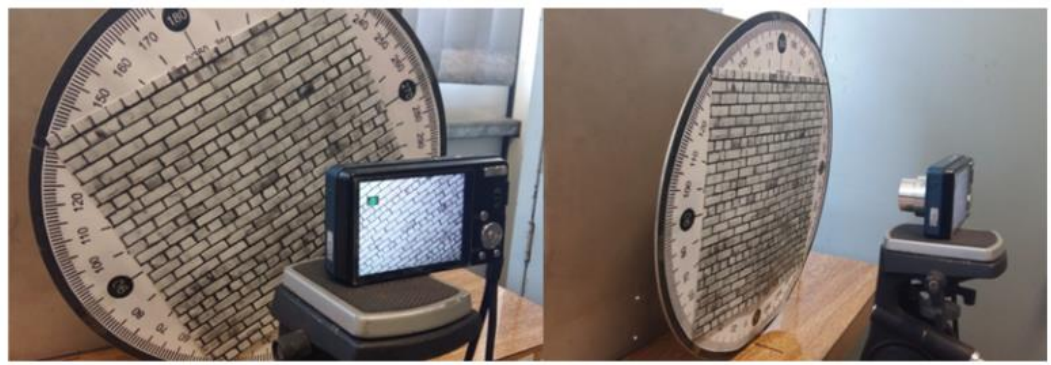

As texturas foram digitalizadas na resolução VGA, resultando em uma imagem de tamanho 640 x 480 pixels e formato JPEG. As imagens foram captadas em dias diferentes e sem nenhum tipo de controle da iluminação. Das 111 classes de texturas do Álbum de Brodatz, 32 classes foram escolhidas aleatoriamente, impressas e digitalizadas. A Figura 5.5 exibe uma imagem para cada classe selecionada. 
Figura 5.5 Base de imagens com 32 texturas do Álbum de Brodatz.
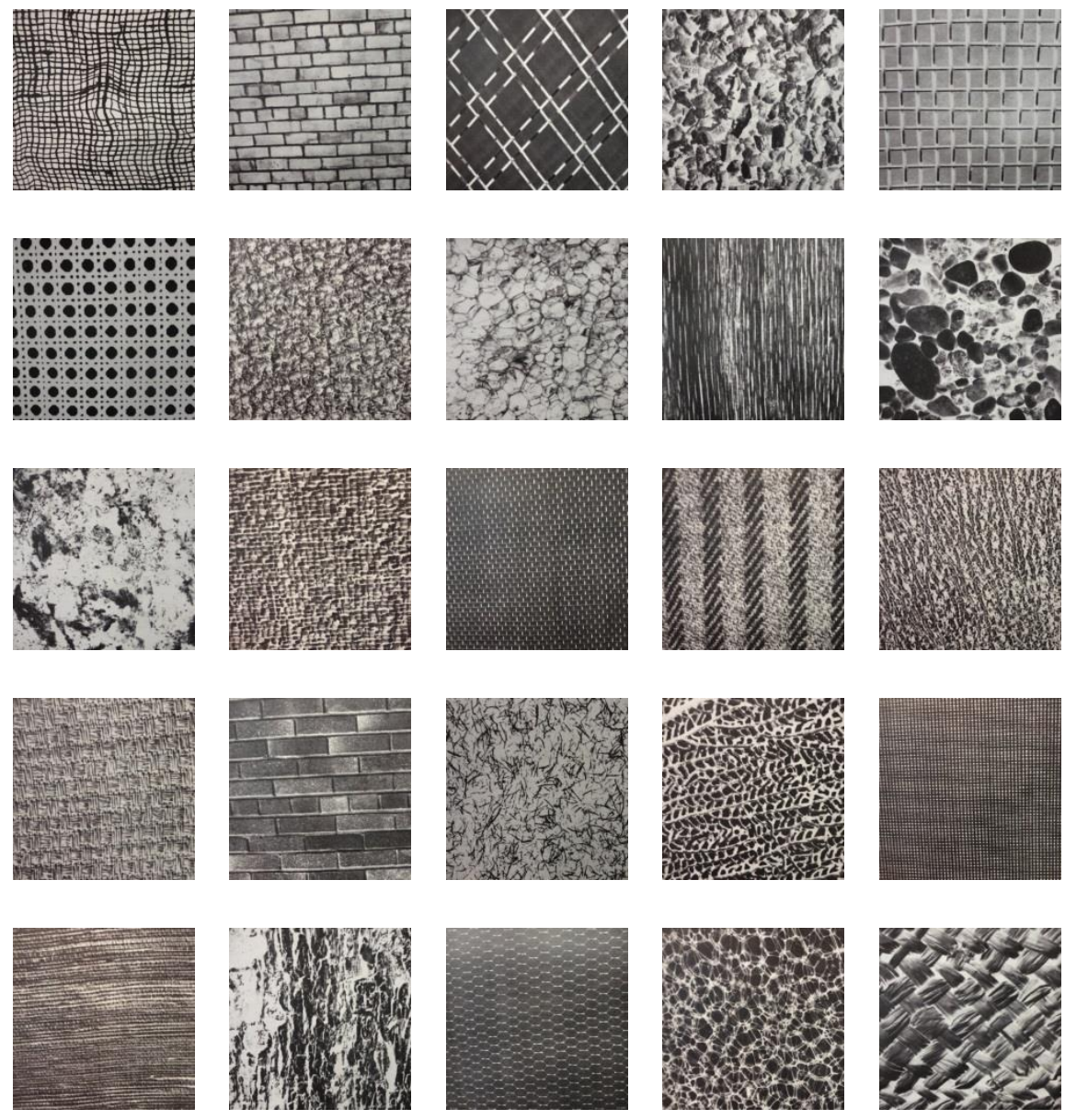
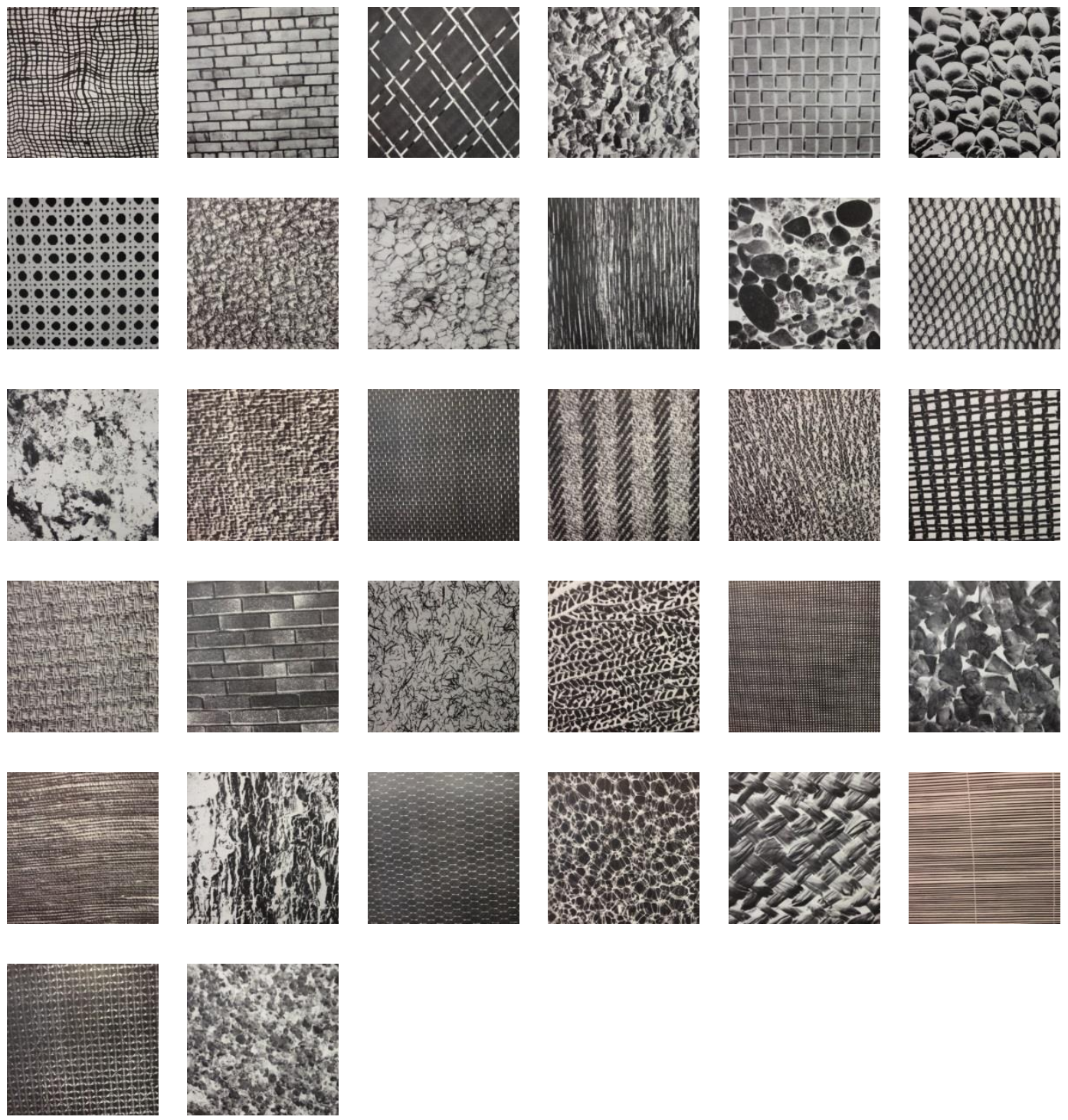

Para cada classe foi realizada a captura de imagens em 21 ângulos de rotação: $0^{\circ}, 10^{\circ}$, $20^{\circ}, 30^{\circ}, 40^{\circ}, 45^{\circ}, 50^{\circ}, 60^{\circ}, 70^{\circ}, 80^{\circ}, 90^{\circ}, 100^{\circ}, 110^{\circ}, 120^{\circ}, 130^{\circ}, 135^{\circ}, 140^{\circ}, 150^{\circ}, 160^{\circ}, 170^{\circ} \mathrm{e}$ $180^{\circ}$. Na Figura 5.6 é exibido um exemplo de uma textura do banco digitalizada nos 21 ângulos de rotação propostos. 
Figura 5.6 Exemplo de digitalização de textura em 21 ângulos de rotação.

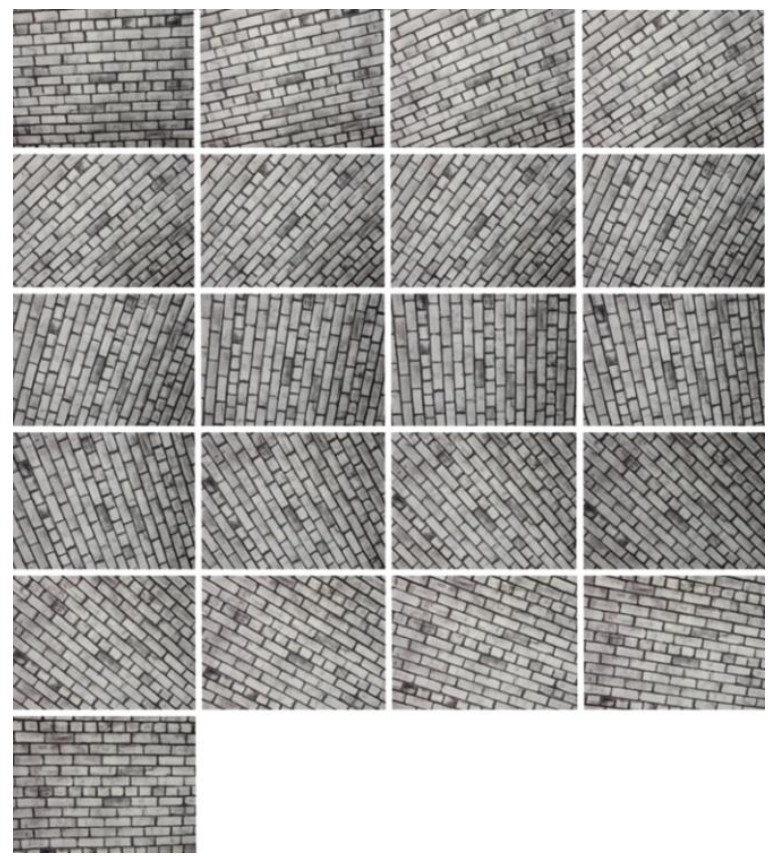

Após o processo de captura das imagens rotacionadas foram extraídas 50 amostras aleatórias (Figura 5.7) de cada classe (podendo haver sobreposição) em cada ângulo de rotação com diferentes tamanhos 72 × 72 pixels, 92 × 92 pixels, 112 × 112 pixels e $132 \times 132$ pixels, formando um conjunto total de 134.400 amostras.

Figura 5.7 Extração de amostras aleatórias da imagem de textura rotacionada.

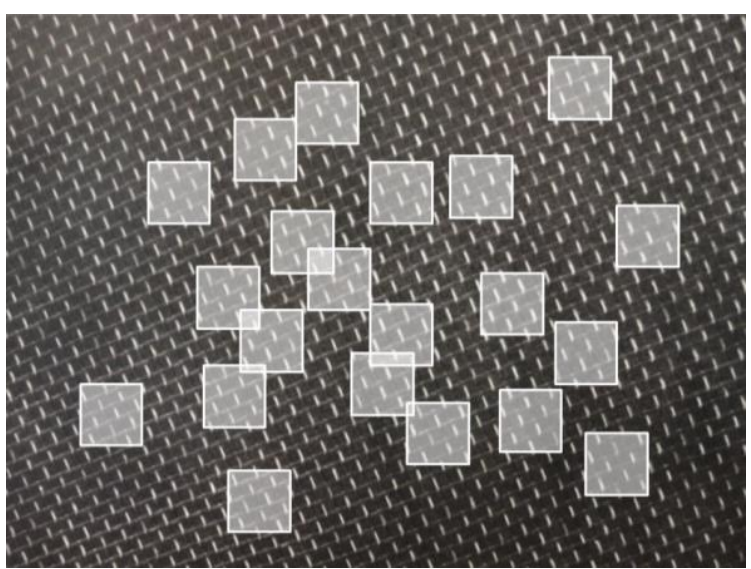

\subsubsection{Rotação por software}

A rotação por software foi conduzida da mesma forma realizada na base Kylberg Sintorn com o toolbox DIPimage ${ }^{4}$ utilizando os métodos de interpolação: Nearest Neighbor,

\footnotetext{
${ }^{4}$ http://www.diplib.org/
} 
Linear, Cúbica de terceira ordem, cúbica B-spline e Lanczos. Assim como nas imagens obtidas pela rotação por hardware foram geradas imagens para os 21 ângulos de rotação e extraídas amostras com 72 x 72 pixels, 92 x 92 pixels, 112 × 112 pixels e 132 × 132 pixels. Assim, para cada uma das cinco interpolações aplicadas foi gerado um conjunto de 134.400 imagens.

\subsection{Processo de avaliação e classificação}

Os métodos de interpolação e os descritores de textura são avaliados comparando-se a acurácia de classificação ou o número de classificações corretas. Para comparação dos vetores de características de cada descritor é usado o classificador First Nearest Neighbor (1-NN) com a métrica de distância Euclidiana (Equação 5.9),

$$
\operatorname{dEuclidiana}(p, q)=\sum_{i=1}^{M} \sqrt{\left(p_{i}-q_{i}\right)^{2}}
$$

em que, $p_{i}$ e $q_{i}$ representam os vetores de características de duas amostras distintas com $M$ elementos.

Para validar o classificador treinado foi realizada a validação cruzada $k$-fold com $k=10$ com atribuição aleatória a cada amostra de textura um índice $n \in\{1,2,3, \ldots 10\}$ criando assim, 10 subcojuntos disjuntos. Na primeira validação cruzada, amostras com índice $n \in\{2,3, \ldots 10\}$ formam o conjunto de treinamento e amostras com índice $n=1$ formam o conjunto de teste. Na segunda validação cruzada, amostras com índice $n=2$ constituem o conjunto de teste e as demais amostras formam o conjunto de treinamento, e assim por diante, conforme ilustrado na Figura 5.8. 
Figura 5.8 Processo de classificação.
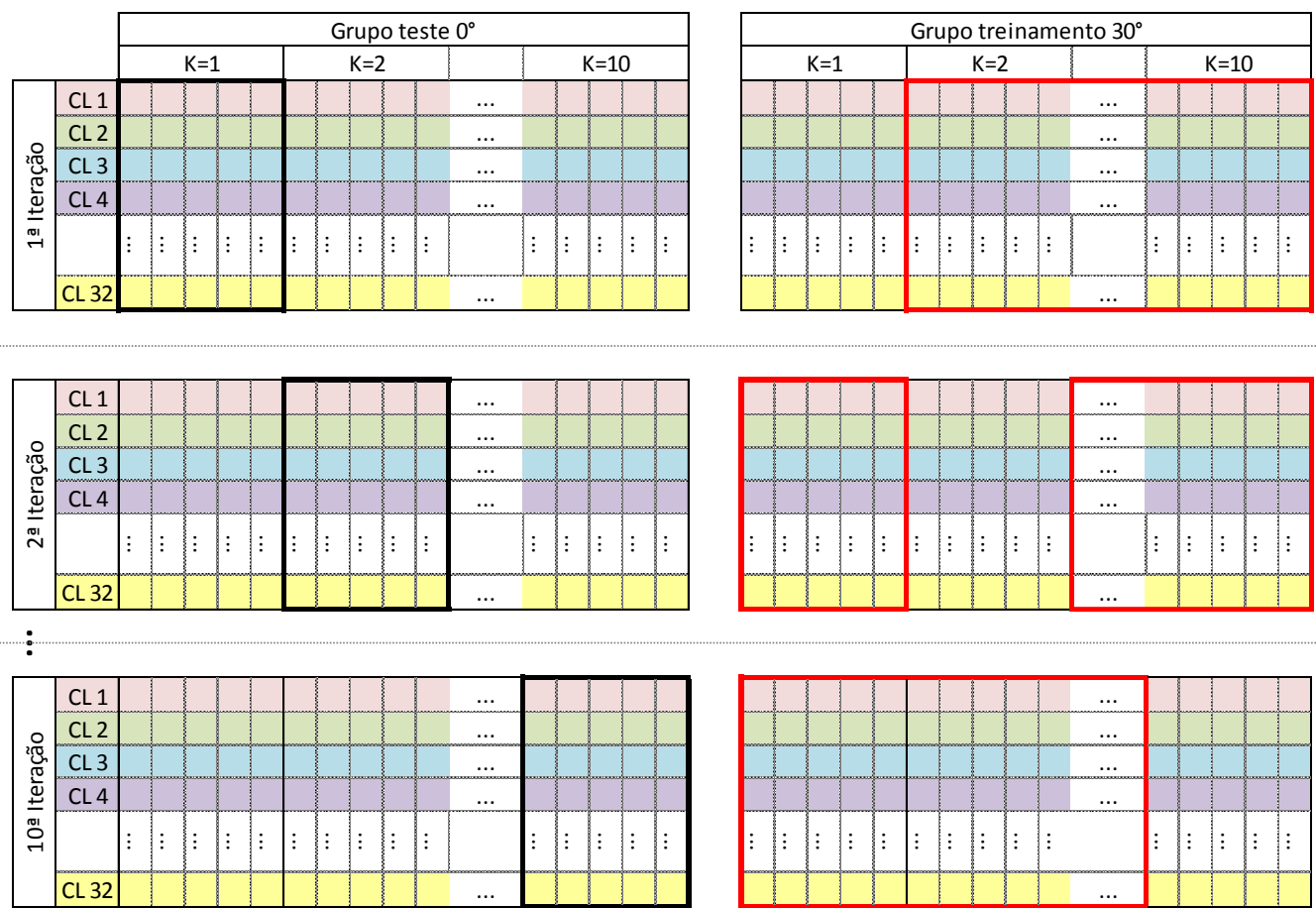

Os resultados das classificações são armazenados em uma matriz de confusão e é feito o cálculo da acurácia média das iterações alcançadas por cada descritor.

\subsection{Considerações Finais}

Neste capítulo foi apresentada a metodologia utilizada para otimização dos parâmetros de descritores baseados no descritor paramétrico LMP. Foi apresentada também a metodologia aplicada para avaliação dos descritores e uma nova base rotacionada do Álbum de Brodatz com maior variedade de ângulos de rotação, tamanhos de amostras e diferentes métodos de rotação. No capítulo seguinte são exibidos e discutidos os resultados obtidos que validam a metodologia apresentada no presente capítulo. 


\section{Capítulo 6}

\section{Resultados}

\subsection{Considerações iniciais}

Neste capítulo são apresentados os resultados experimentais obtidos ao aplicar os descritores de micropadrões CLMP, SLMP_M propostos nesta tese e comparados com os descritores estado da arte CLBP, LBP ${ }^{\text {DFT }}$, ILBP ${ }^{\text {DFT }}$, LTP ${ }^{\text {DFT }}$, ILTP ${ }^{\text {DFT }}$, na classificação de imagens de textura. Em seguida, os descritores são avaliados em cinco experimentos realizando-se testes computacionais em imagens rotacionadas das bases Kylberg Syntorn Rotation Dataset (KYLBERG, 2014) e Brodatz Texture Rotation Dataset e são verificadas as limitações e desempenhos de cada um.

Para condução dos experimentos com os descritores paramétricos propostos nesta tese foi necessário um ajuste prévio dos parâmetros $\beta$ e $B$. Na Tabela 6.1 estão os parâmetros ajustados para a base de imagens Kylberg Sintorn. Para este ajuste utilizou-se $50 \%$ das amostras com tamanho $120 \times 120$ pixels.

Tabela 6.1 Parâmetros ótimos para a base Kylberg Sintorn

\begin{tabular}{lcccc} 
& \multicolumn{5}{c}{ Parâmetros } \\
\hline \multicolumn{1}{c}{ Descritor } & $\beta_{S}$ & $\beta_{M}$ & BM & Bs \\
\hline \hline CLMP_M/C $(8,1)$ & 1,6216 & 0,6943 & 75 & ----- \\
CLMP_M/C $(16,2)$ & 2,0554 & 1,7359 & 81 & ---- \\
CLMP_M/C $(24,3)$ & 2,0652 & 1,5266 & 101 & ---- \\
CLMP_S_M/C $(8,1)$ & 1,0031 & 0,5417 & 159 & 41 \\
CLMP_S_M/C $(16,2)$ & 1,5924 & 1,1948 & 171 & 32 \\
CLMP_S_M/C $(24,3)$ & 1,9811 & 0,9982 & 128 & 49 \\
CLMP_S/M (8,1) & 0,7089 & 0,4768 & 120 & 160 \\
CLMP_S/M (16,2) & 1,8287 & 0,4900 & 155 & 197 \\
CLMP_S/M (24,3) & 2,4470 & 0,4503 & 172 & 172 \\
CLMP_S/M/C (8,1) & 0,6477 & 0,7336 & 107 & 51 \\
CLMP_S/M/C (16,2) & 1,0319 & 0,4778 & 58 & 73 \\
CLMP_S/M/C (24,3) & 0,6987 & 0,5257 & 32 & 62 \\
\hline \multicolumn{1}{c}{ Descritor } & $\beta_{1}$ & $\beta_{2}$ & B1 & B2 \\
\hline \hline SLMP_M (8,1) & 2,0579 & 0,5418 & 128 & 83 \\
SLMP_M (16,2) & 1,8923 & 0,4974 & 122 & 107 \\
SLMP_M (24,3) & 2,0093 & 0,7154 & 112 & 62 \\
\hline
\end{tabular}


A Tabela 6.2 exibe os parâmetros para a base de imagens Brodatz Texture Rotation Dataset que também foram ajustados em $50 \%$ das amostras, mas com tamanho $90 \times 90$ pixels.

Tabela 6.2 Parâmetros ótimos para a base Brodatz Texture Rotation Dataset

\begin{tabular}{lcccc} 
& \multicolumn{5}{c}{ Parâmetros } \\
\hline \multicolumn{1}{c}{ Descritor } & $\beta_{S}$ & $\beta_{M}$ & BM & Bs \\
\hline \hline CLMP_M/C $(8,1)$ & 2,2392 & 0,7886 & 171 & ----- \\
CLMP_M/C (16,2) & 2,4837 & 0,6714 & 149 & ----- \\
CLMP_M/C (24,3) & 2,1178 & 1,0478 & 112 & ---- \\
CLMP_S_M/C (8,1) & 1,5534 & 0,4204 & 105 & 32 \\
CLMP_S_M/C (16,2) & 2,4192 & 0,9290 & 183 & 30 \\
CLMP_S_M/C (24,3) & 1,9806 & 0,5512 & 179 & 33 \\
CLMP_S/M (8,1) & 1,1793 & 0,4146 & 164 & 130 \\
CLMP_S/M (16,2) & 2,4470 & 0,4503 & 172 & 172 \\
CLMP_S/M (24,3) & 0,7932 & 0,4628 & 188 & 195 \\
CLMP_S/M/C (8,1) & 0,8075 & 0,4106 & 131 & 142 \\
CLMP_S/M/C (16,2) & 1,8459 & 0,4571 & 174 & 188 \\
CLMP_S/M/C (24,3) & 0,7932 & 0,4628 & 188 & 195 \\
\hline \multicolumn{1}{c}{ Descritor } & $\beta_{1}$ & $\beta_{2}$ & B1 & B2 \\
\hline \hline SLMP_M (8,1) & 1,2785 & 1,8893 & 106 & 42 \\
SLMP_M (16,2) & 1,0060 & 1,7630 & 186 & 41 \\
SLMP_M (24,3) & 0,9871 & 1,2711 & 178 & 50 \\
\hline
\end{tabular}

\subsection{Experimento 1}

No trabalho de Kylberg e Sintorn (2016) são propostos os descritores $\operatorname{LTP}^{\text {DFT }}$ e ILTP $^{\text {DFT }}$ e comparados com os LBP $^{\text {DFT }}$, ILBP ${ }^{\text {DFT }}$, que combinam a versão generalizada das características de Fourier (FERNANDEZ, 2011), e alcançam a invariância à rotação com os descritores LTP, ILTP, LBP e ILBP, respectivamente. Os descritores avaliados utilizam apenas a configuração de raio $\mathrm{R}=1$ e número de pontos de amostragem $\mathrm{P}=8$, pois, para os descritores propostos, $\mathrm{P} \gg 8$ resulta em um espaço de características com alta dimensionalidade, por exemplo $\mathrm{LBP}_{16,1}^{\mathrm{DFT}} \in \mathbb{R}^{36.883}$ e $\operatorname{ILTP}_{16,1}^{\mathrm{DFT}} \in \mathbb{R}^{147.532}$ e isto acarreta em um vetor de características muito grande e, consequentemente, um aumento do custo computacional.

Neste experimento foi reproduzido o mesmo teste realizado no trabalho de Kylberg e Sintorn (2016) avaliando-se 100 amostras de tamanho 120 x 120 pixels para cada classe de textura da base. A Tabela 6.3 apresenta a dimensão de todos os descritores avaliados neste experimento. 
Tabela 6.3 Dimensão dos descritores avaliados com configuração $(8,1)$

\begin{tabular}{lc}
\hline \multicolumn{1}{c}{ Descritor } & Dimensão \\
\hline \hline LTPDFT & 326 \\
\hline ILTPDFT & 651 \\
\hline ILBPDFT & 325 \\
\hline LBPDFT & 163 \\
\hline SLMP_M & 211 \\
\hline CLMP_M/C & 20 \\
\hline CLMP_S_M/C & 30 \\
\hline CLMP_S/M & 100 \\
\hline CLMP_S/M/C & 200 \\
\hline CLBP_M & 10 \\
\hline CLBP_S & 10 \\
\hline CLBP_M/C & 20 \\
\hline CLBP_S_M/C & 30 \\
\hline CLBP_S/M & 100 \\
\hline CLBP_S/M/C & 200 \\
\hline
\end{tabular}

Os gráficos das Figuras 6.1 a 6.6 exibem os resultados obtidos com as medidas de acurácia média e desvio padrão ao longo dos ângulos da base Kylberg Syntorn Rotation Dataset (KYLBERG, 2014) tanto com imagens rotacionadas por hardware como por software. Os resultados obtidos para cada ângulo separadamente podem ser visualizados nas tabelas no Apêndice A.

Figura 6.1 Acurácia média e desvio padrão para imagem rotacionada por Hardware

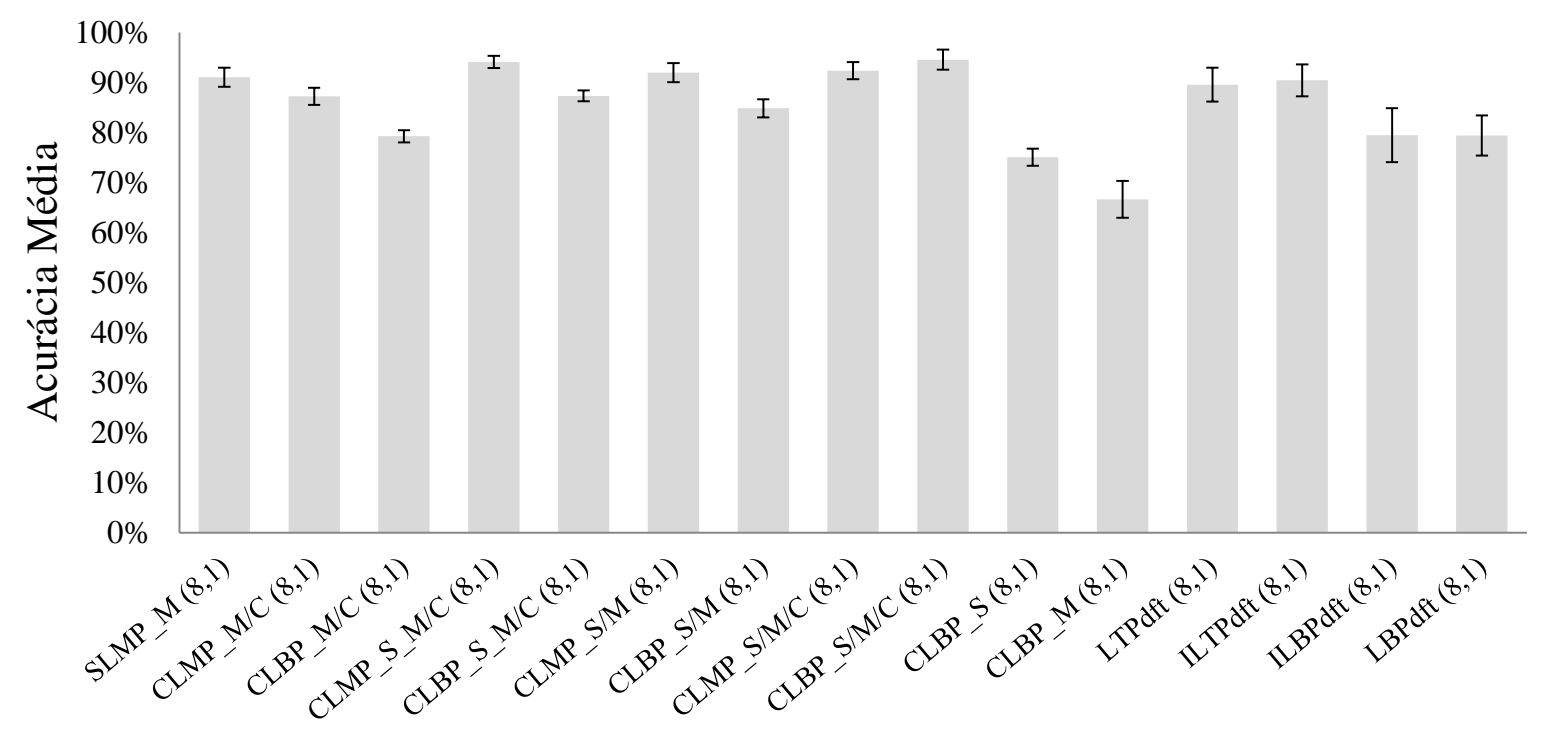


Figura 6.2 Acurácia média e desvio padrão para imagem rotacionada pela interpolação B-spline

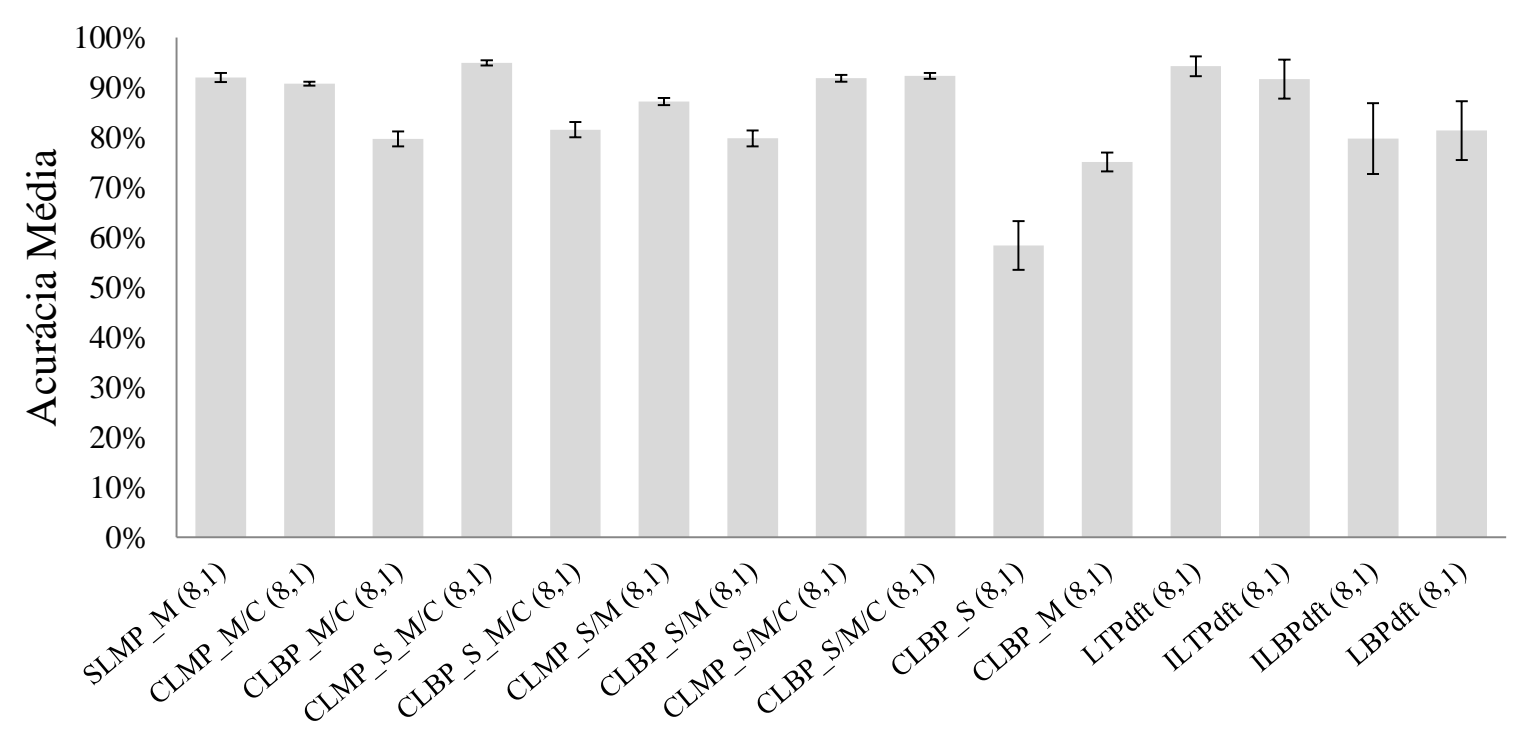

Figura 6.3 Acurácia média e desvio padrão para imagem rotacionada pela interpolação Lanczos

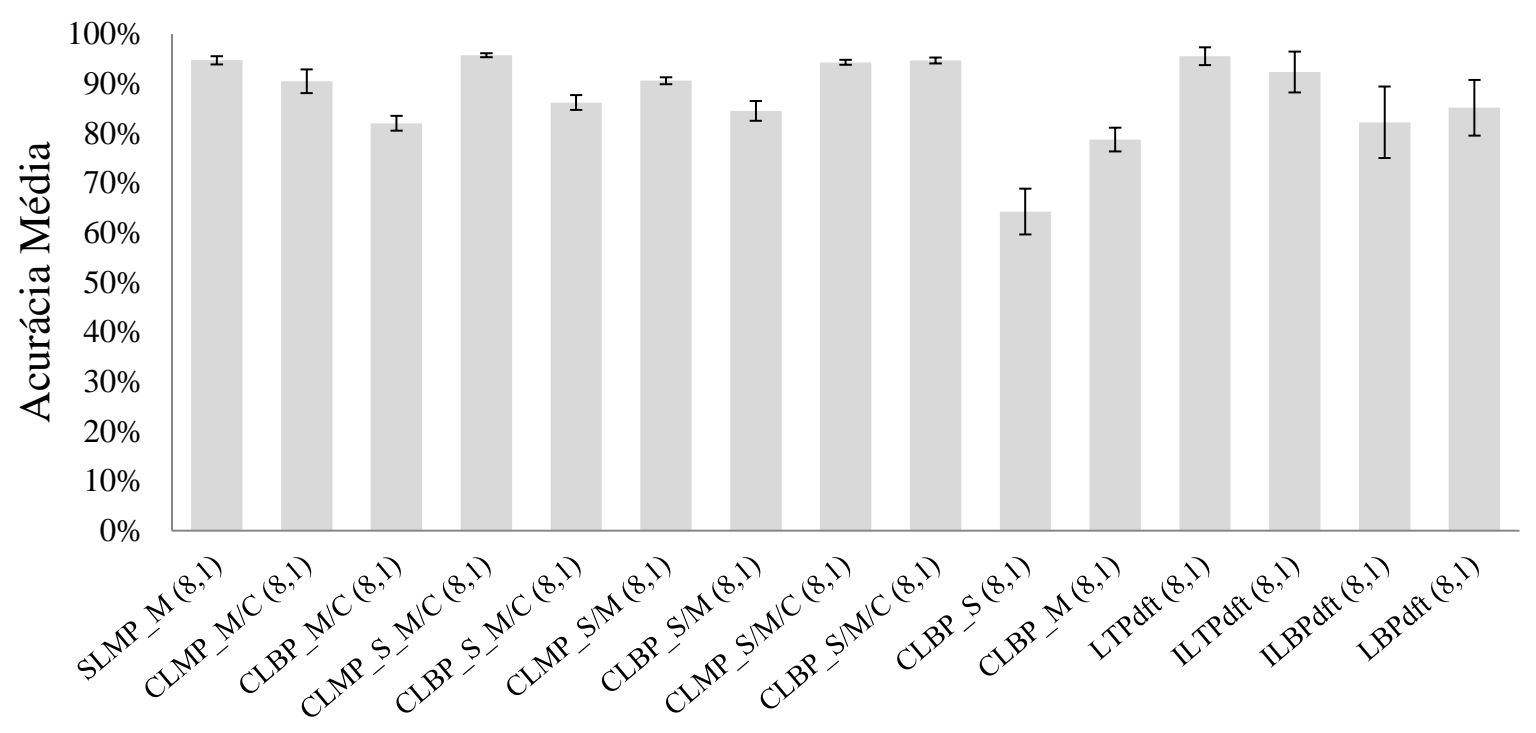

Ao analisar a robustez de cada descritor em cada método de interpolação, observa-se que os resultados de melhor desempenho são devido aos métodos Lanczos e B-spline. Eles têm precisões semelhantes, até melhor do que a obtida para texturas rotacionadas por hardware. Isso pode, de alguma forma, ser explicado pelo fato que, durante as rotações realizadas por hardware, o ruído do sensor é amostrado repetidamente em diferentes direções, enquanto que, as imagens interpoladas, são geradas a partir de uma imagem cujo ruído do sensor é amostrado apenas uma vez. Assim, o conjunto de imagens giradas por interpolação é mais homogêneo. 
Figura 6.4 Acurácia média e desvio padrão para imagem rotacionada pela interpolação Linear

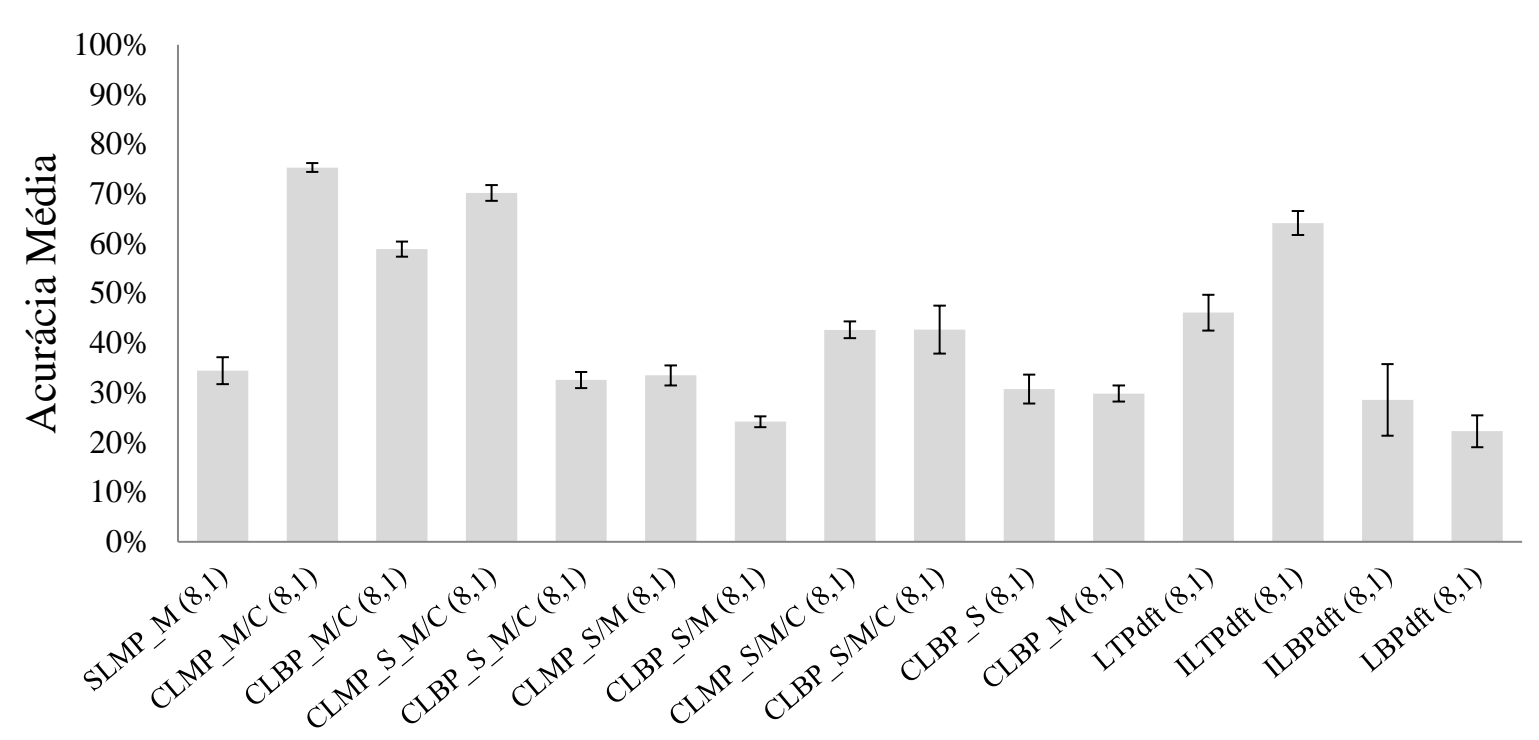

Figura 6.5 Acurácia média e desvio padrão para imagem rotacionada pela interpolação Cúbica

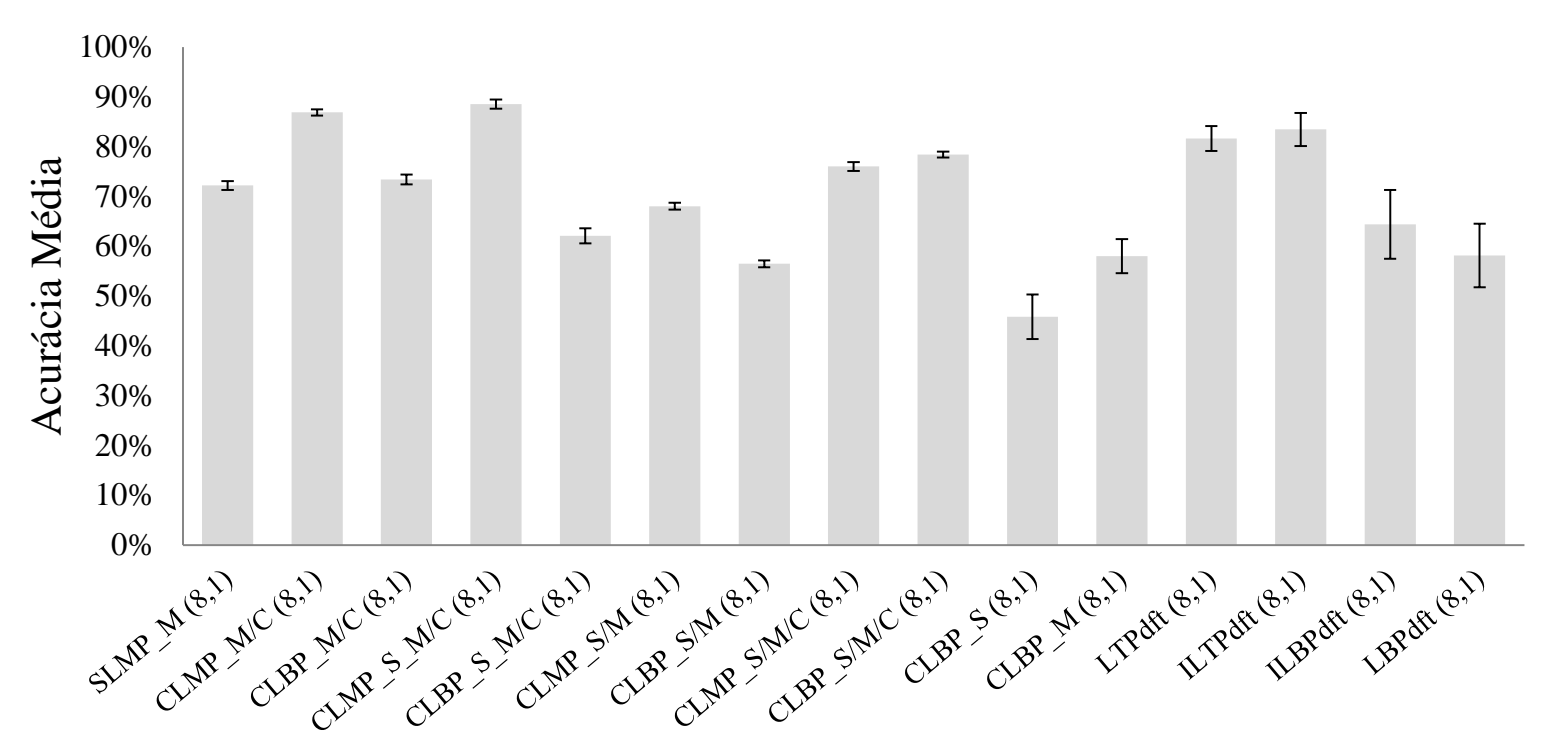

Os melhores resultados são obtidos pelos descritores CLMP_S_M/C $(8,1)$ e $\operatorname{LTPDFT}(8,1)$ ao aplicar a interpolação Lanczos. Apesar de ambos alcançarem uma acurácia média de 96,0\%, o desvio padrão do descritor CLMP_S_M/C é de apenas 0,004 enquanto que o apresentado pelo descritor LTPDFT é de 0,018 . Isto demonstra que há uma maior robustez do descritor proposto em relação à variação dos ângulos. Esta é uma característica muito importante já que, um descritor para ser considerado invariante à rotação deve mostrar variações sutis sob diferentes orientações de uma textura não sendo dependente do ângulo da textura. Isto é, um descritor que apresente um excelente desempenho para um determinado ângulo e decai para outros é extremamente dependente de conhecimento prévio sobre os ângulos avaliados, o que não é uma condição normal para imagens reais. 
Figura 6.6 Acurácia média e desvio padrão para imagem rotacionada pela interpolação Nearest Neighbor

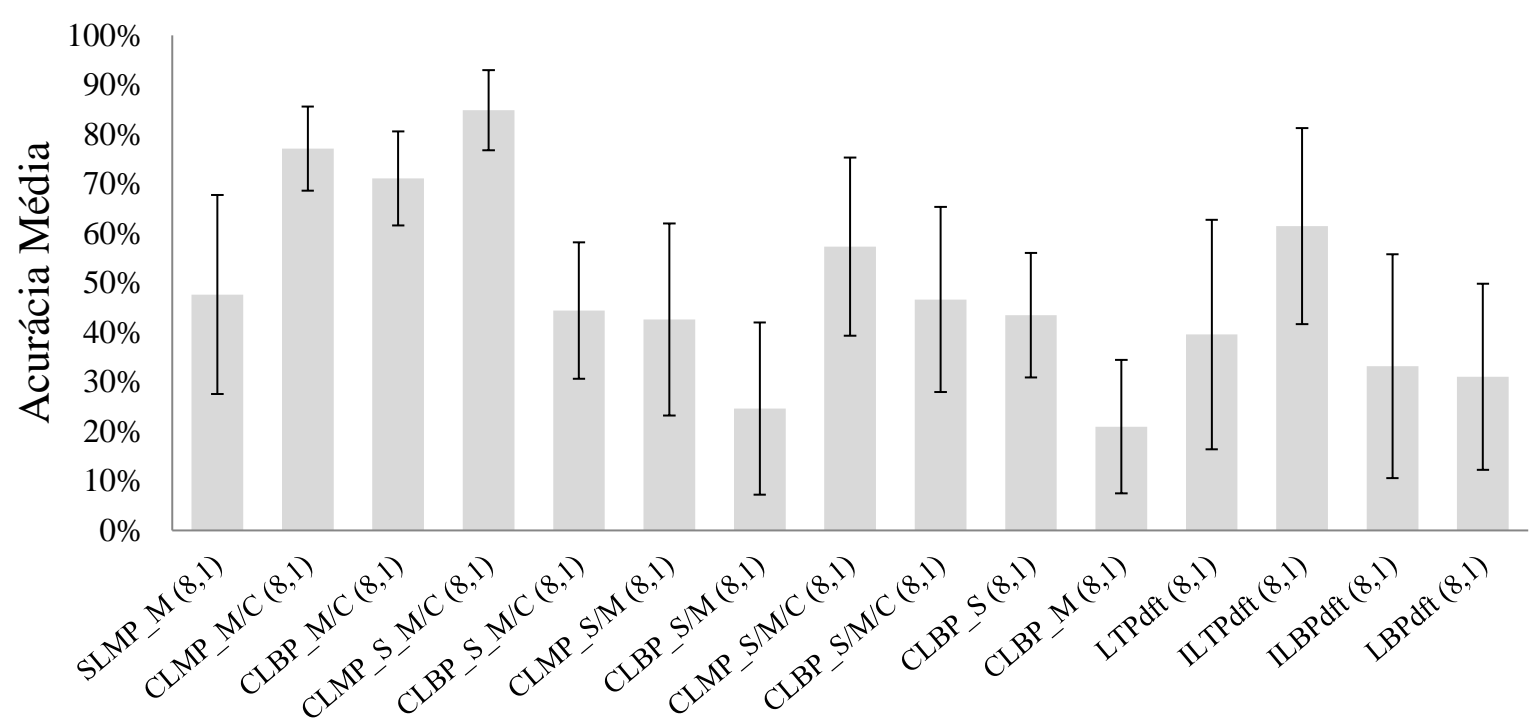

As demais interpolações Cúbica, Linear e Nearest Neighbor não apresentaram bom desempenho em manter as informações da imagem rotacionada. Observa-se que na interpolação Nearest Neighbor o desvio padrão é o mais alto entre todas, mostrando-se uma interpolação não adequada.

\subsection{Experimento 2}

Um segundo experimento com a base Kylberg Sintorn foi realizado utilizando diferentes configurações de raio e pontos de amostragem para as amostras com tamanho $120 \mathrm{x}$ 120 pixels. Este teste não foi realizado no trabalho de Kylberg por conta do aumento da dimensionalidade do vetor de característica, conforme apontado pelos autores. A Tabela 6.4 exibe a dimensão dos descritores avaliados neste experimento com duas diferentes configurações de raio e pontos de amostragem. Os descritores CLBP_S e CLBP_M não apresentaram bom desempenho no experimento $1 \mathrm{e}$, por isto, não foram inseridos neste teste. Devido à impossibilidade de obtenção dos códigos originais dos descritores baseados nas características de Fourier, estes também não foram inseridos neste experimento. 
Tabela 6.4 Dimensão dos descritores avaliados com duas configurações de pontos de amostragem e raio

\begin{tabular}{lcc} 
& \multicolumn{2}{c}{ (Pontos, Raio) } \\
\hline \multicolumn{1}{c}{ Descritor } & $(16,2)$ & $(24,3)$ \\
\hline \hline SLMP_M & 229 & 174 \\
CLMP_M/C & 36 & 52 \\
CLMP_S_M/C & 54 & 78 \\
CLMP_S/M & 324 & 676 \\
CLMP_S/M/C & 648 & 1352 \\
CLBP_M/C & 36 & 52 \\
CLBP_S_M/C & 54 & 78 \\
CLBP_S/M & 324 & 676 \\
CLBP_S/M/C & 648 & 1352 \\
\hline
\end{tabular}

Os gráficos das Figuras 6.7 a 6.12 apresentam uma análise comparativa da média da acurácia para diferentes configurações e métodos de rotação aplicados nas imagens. Tabelas com os valores detalhados para cada descritor e cada configuração podem ser verificadas no Apêndice B.

Figura 6.7 Avaliação dos descritores com duas configurações (P,R) em imagens rotacionadas por Hardware

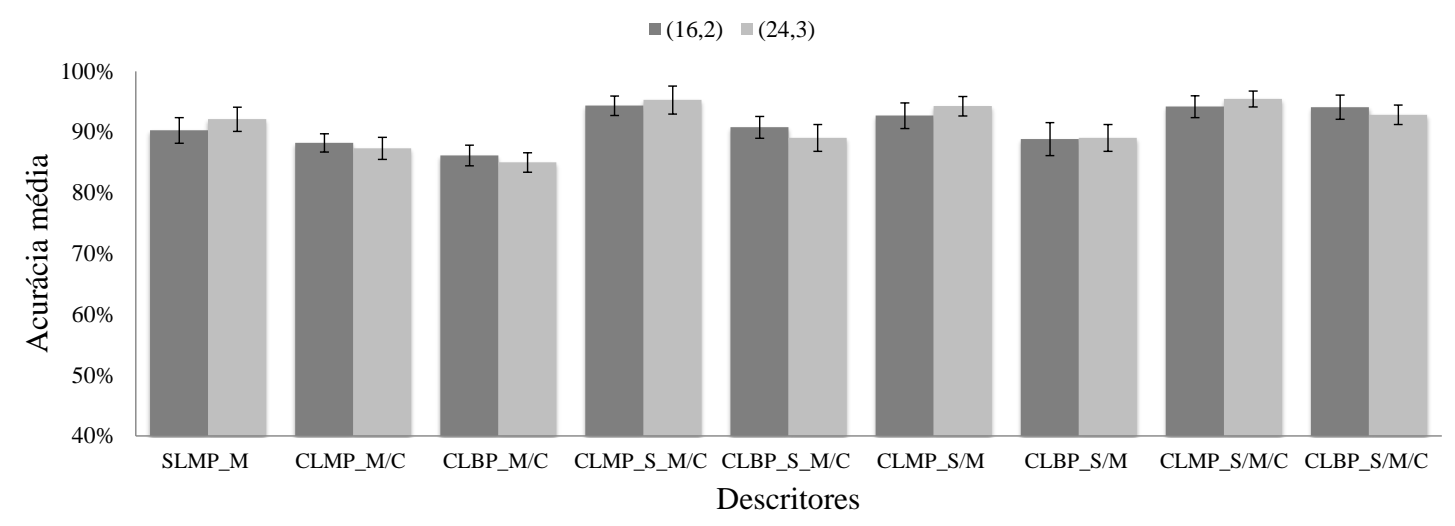

Figura 6.8 Avaliação dos descritores com duas configurações $(\mathrm{P}, \mathrm{R})$ em imagens rotacionadas por interpolação B-spline

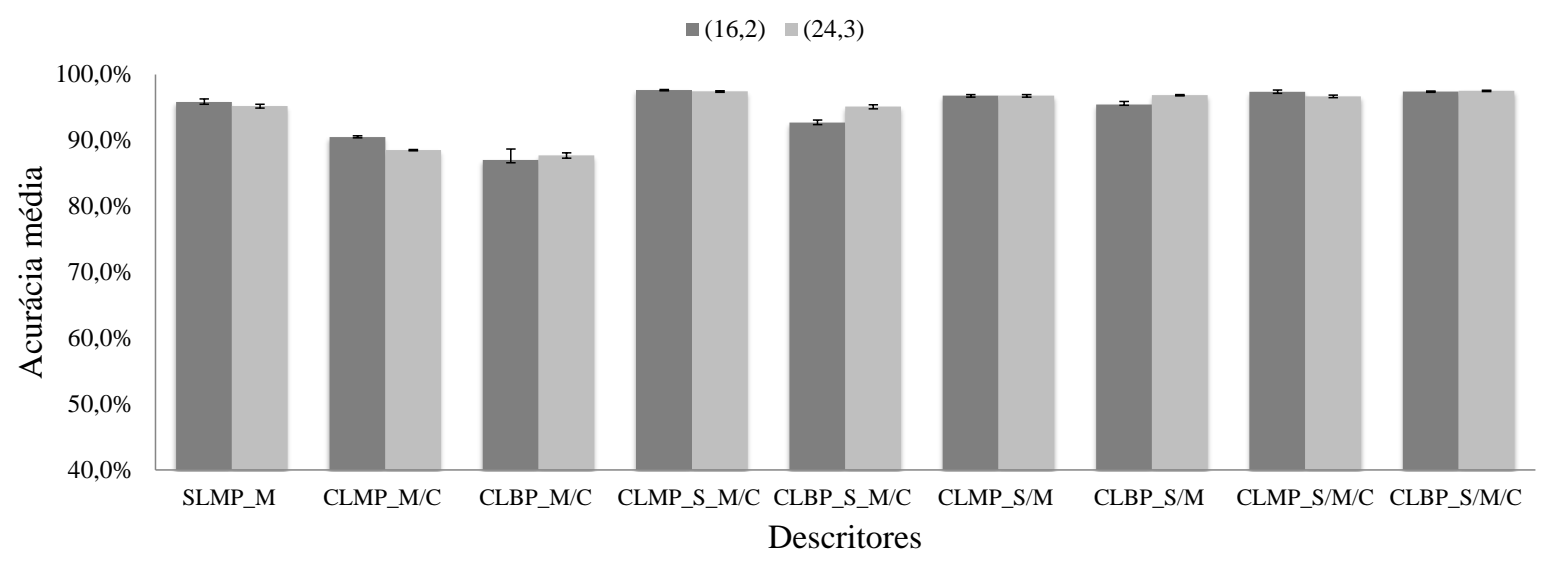


Figura 6.9 Avaliação dos descritores com duas configurações $(\mathrm{P}, \mathrm{R})$ em imagens rotacionadas por interpolação Lanczos

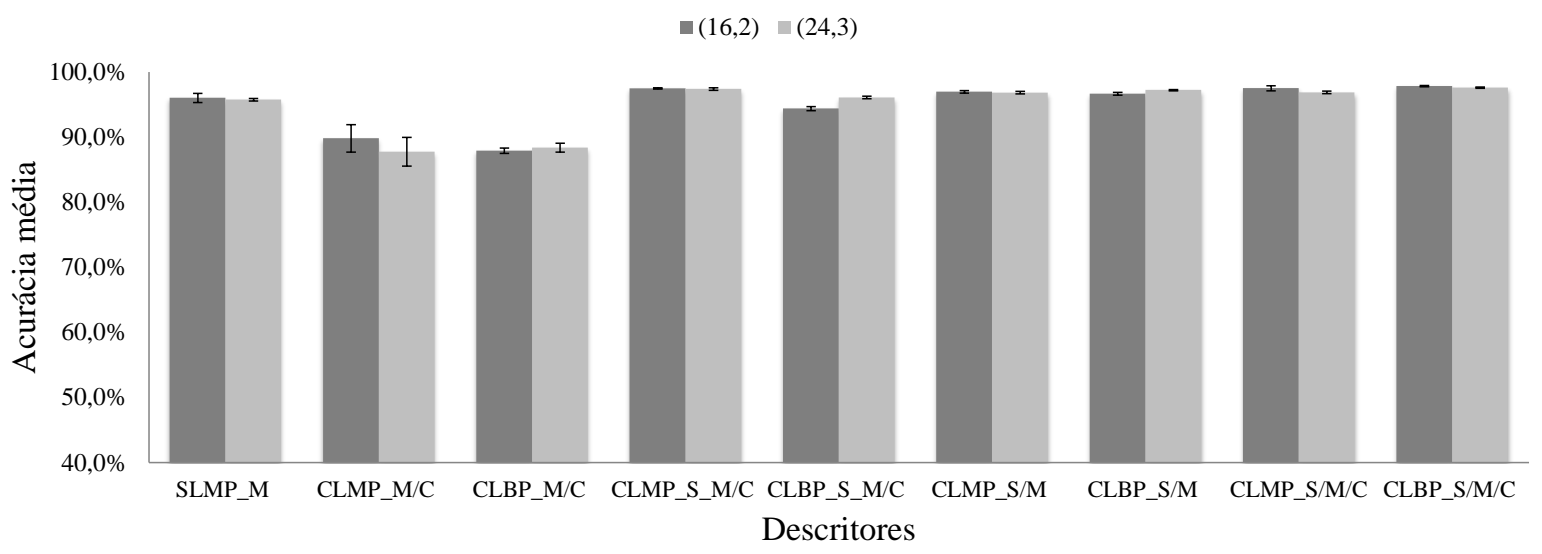

Nos testes realizados com as imagens rotacionadas por Hardware e pelas interpolações B-spline e Lanczos, o aumento dos pontos de amostragem de 16 para 24 e o raio de 2 para 3 não promoveram um ganho significativo de acurácia. Em alguns descritores inclusive, a configuração $(16,2)$ apresenta melhor acurácia média em relação à configuração $(24,3)$ como, por exemplo, o descritor SLMP_M na interpolação Lanczos com a configuração SLMP_M(16,2) que apresenta uma acurácia média de 96,0\% e, em sua configuração SLMP_M(24,3), uma acurácia de 95,7\%. Já para o descritor CLMP_M/C na interpolação Bspline a diferença é ainda maior pois apresenta $2 \%$ a menos no valor da acurácia média ao aumentar o raio e a quantidade de pontos de amostragem, ou seja, nem sempre o aumento da quantidade de pontos de amostragem é necessário para uma boa descrição do micropadrão.

Apesar do aumento dos vetores de características, os descritores apresentaram um ganho em relação à configuração $(8,1)$, é o caso do descritor CLMP_S_M/C $(8,1)$ que alcançou uma acurácia média de 96,0\% e nas suas configurações CLMP_S_M/C $(16,2)$ e CLMP_S_M/C (24,3) alcançaram 97,5\% e 97,4\%, respectivamente no teste com imagens com interpolação Lanczos.

Dos demais métodos de interpolação que não obtiveram desempenho superior aos das interpolações B-spline e Lanczos no experimento 1, a interpolação cúbica foi a que melhor apresentou ganhos com o aumento do raio e pontos de amostragem, alcançando $97,1 \%$ de acurácia média com o descritor CLMP_S_M/C com ganho de $8 \%$ em relação a configuração $(8,1)$. 
Figura 6.10 Avaliação dos descritores com duas configurações $(P, R)$ em imagens rotacionadas por interpolação Cúbica

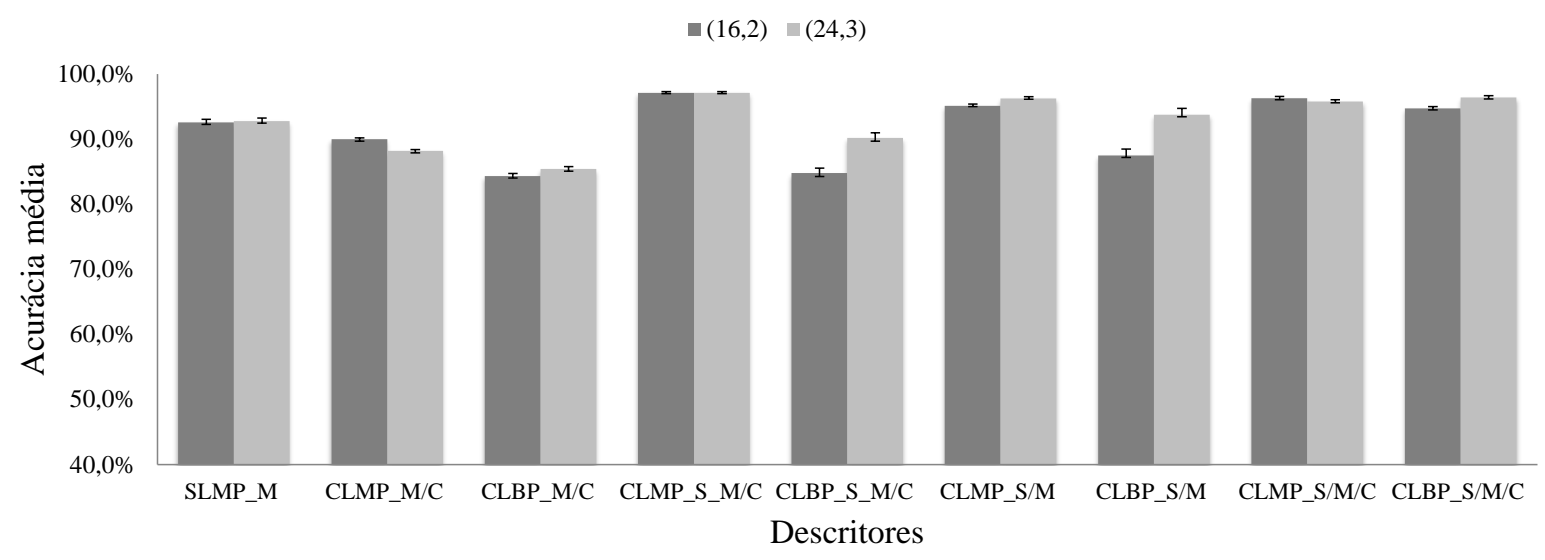

Figura 6.11 Avaliação dos descritores com duas configurações $(P, R)$ em imagens rotacionadas por interpolação Linear

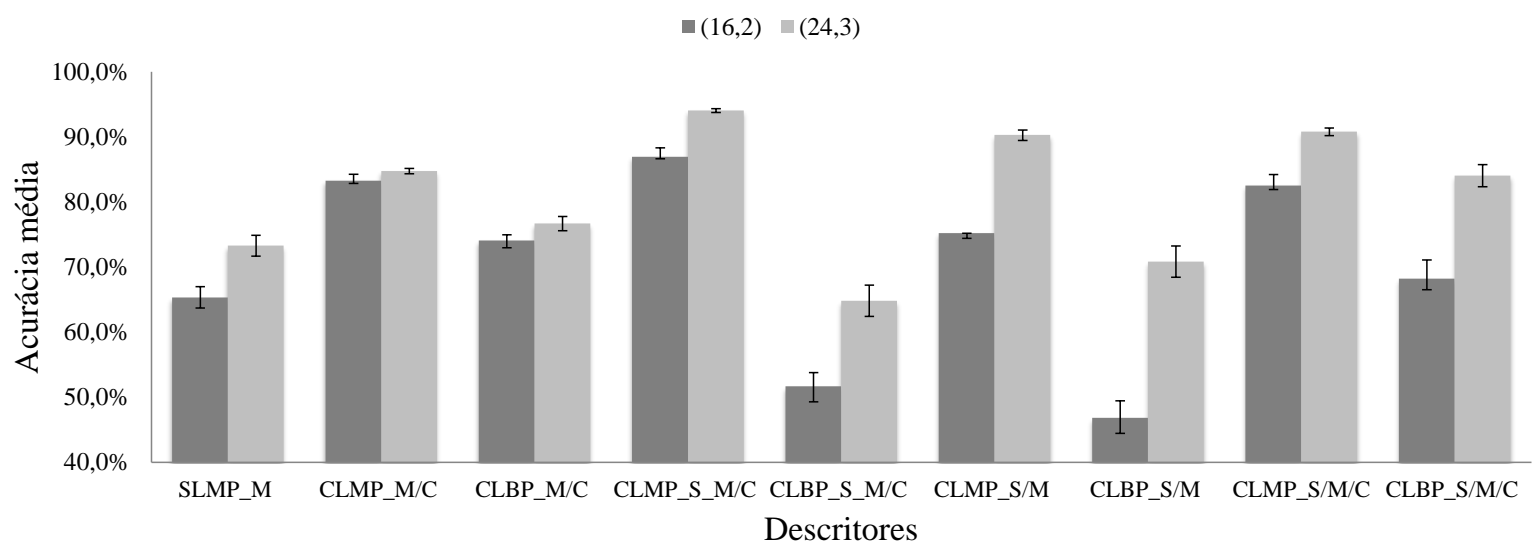

Figura 6.12 Avaliação dos descritores com duas configurações $(\mathrm{P}, \mathrm{R})$ em imagens rotacionadas por interpolação Nearest Neighbor

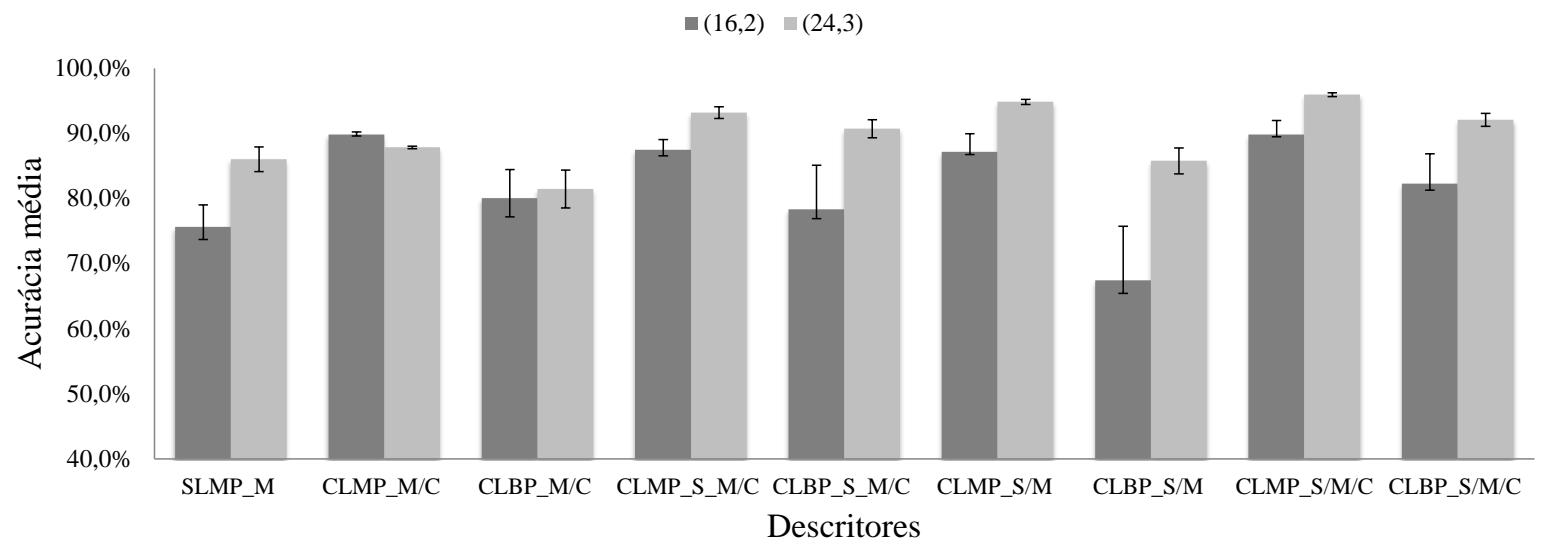




\subsection{Experimento 3}

O experimento 3 consiste na avaliação da influência do tamanho das amostras analisadas no processo classificatório. Os testes são realizados em amostras com tamanho 70 x 70 pixels e 90 x 90 pixels da base Kylberg Sintorn. Os gráficos das Figuras 6.13 a 6.15 exibem uma avaliação dos descritores que apresentaram melhor acurácia média na melhor combinação encontrada para as rotações realizadas por hardware, interpolação B-spline e Lanczos, respectivamente. Os valores detalhados para cada tamanho de amostra e configuração (R, P) dos descritores podem ser verificados no Apêndice C.

Figura 6.13 Acurácia média dos descritores com melhor desempenho em dois diferentes tamanhos de amostras rotacionadas por Hardware
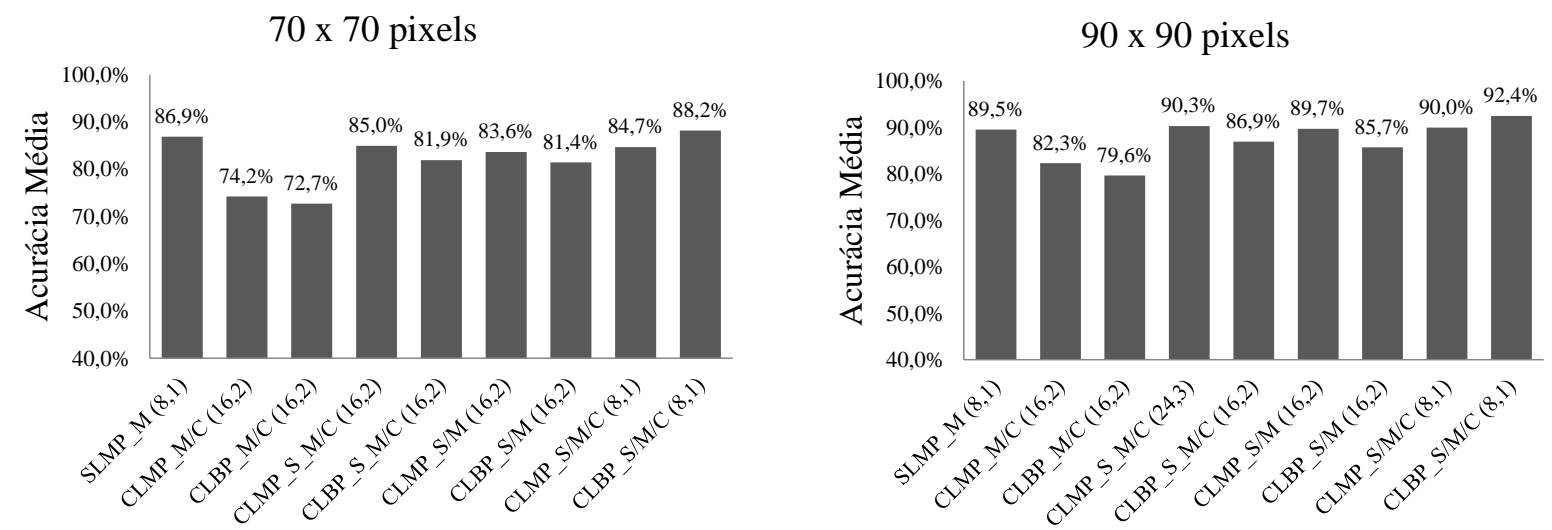

Figura 6.14 Acurácia média dos descritores com melhor desempenho em dois diferentes tamanhos de amostras rotacionadas pela interpolação B-spline
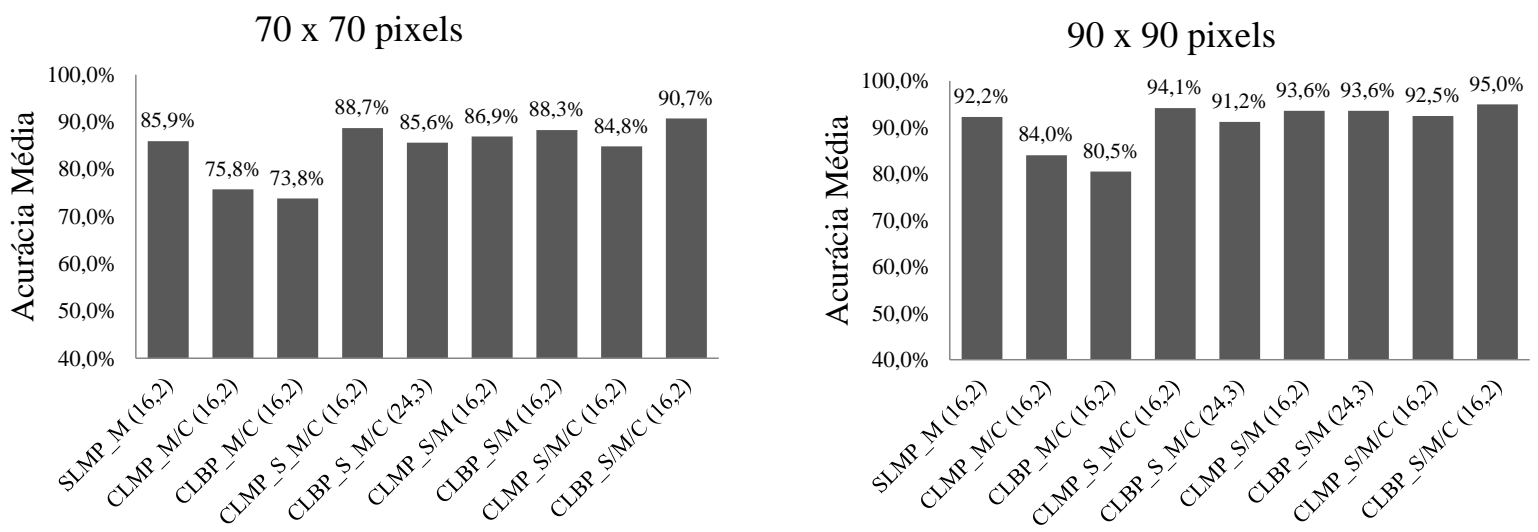
Figura 6.15 Acurácia média dos descritores com melhor desempenho em dois diferentes tamanhos de amostras rotacionadas pela interpolação Lanczos
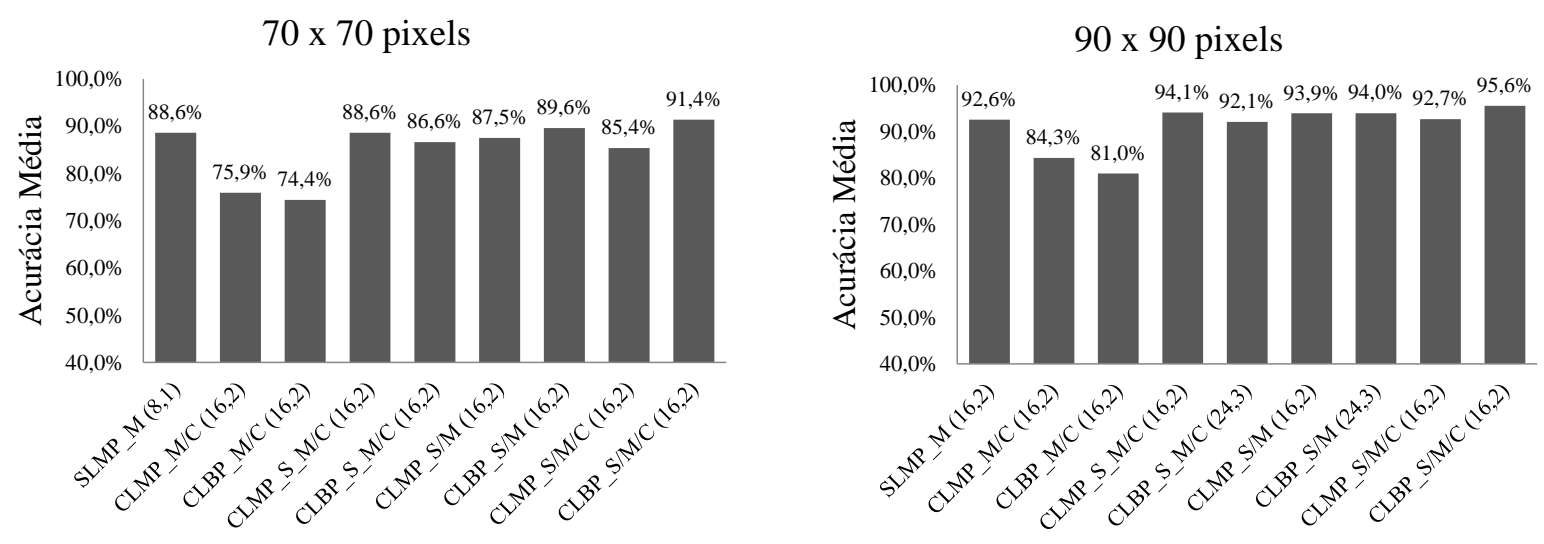

A redução do tamanho das amostras afeta o processo classificatório. Observa-se a queda da acurácia média em todos os descritores avaliados. Isto se justifica pelo fato que a quantidade de informação é reduzida. Assim como nos experimentos anteriores, o melhor desempenho dos descritores é observado nos testes realizados com as imagens rotacionadas por Hardware e pelas interpolações B-spline e Lanczos.

Para amostras menores, os descritores CLBP apresentam melhor acurácia média, porém, é necessário um descritor com o vetor de característica de tamanho bem superior que o necessário para o melhor caso do descritor CLMP. Exceto nos testes realizados com rotação por hardware, as combinações dos operadores CLBP que proporcionam os melhores resultados são CLBP_S/M/C(16,2) com dimensão 648 e CLBP_S/M/C(24,3) com dimensão 1352 e, no caso da rotação por hardware, o CLBP_S/M/C $(8,1)$ com dimensão 200. Para o descritor CLMP, as combinações dos operadores que melhor descrevem as texturas são CLMP_S_M/C(16,2) e CLMP_S_M/C(24,3) com dimensionalidades 54 e 78, respectivamente.

Para este teste com amostras menores, o CLBP apresenta o melhor valor de acurácia média de 95,6\% com amostras de tamanho 90 x 90 pixels na interpolação Lanczos na combinação de operadores CLBP_S/M/C $(16,2)$ e o CLMP_S_M/C $(16,2)$ com 94,1\% de acurácia média. O desempenho do CLBP supera em 1,5\% o CLMP mas necessita de um descritor 12 vezes maior para reter informação discriminante.

Nos demais métodos de interpolação (gráficos das Figuras 6.16 a 6.18) o CLMP apresenta melhor desempenho que o CLBP, chegando a uma diferença de até 7,6\% da acurácia media na interpolação linear de amostras 90 x 90 pixels, por exemplo. 
Figura 6.16 Acurácia média dos descritores com melhor desempenho em dois diferentes tamanhos de amostras rotacionadas pela interpolação Linear
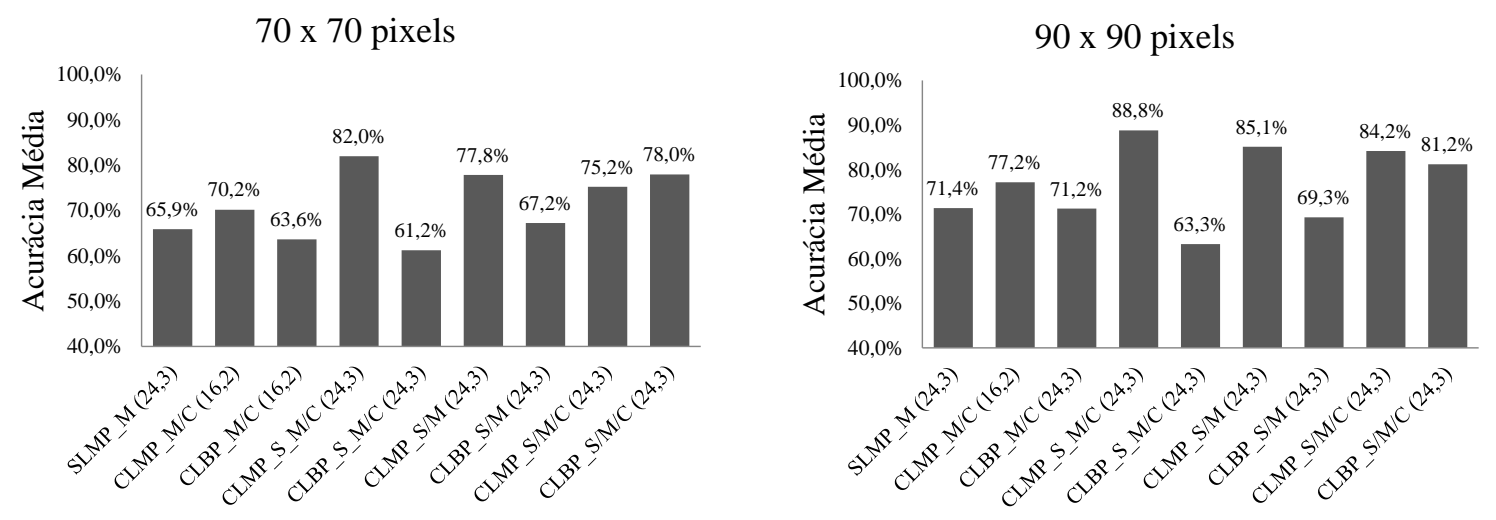

Figura 6.17 Acurácia média dos descritores com melhor desempenho em dois diferentes tamanhos de amostras rotacionadas pela interpolação Cúbica
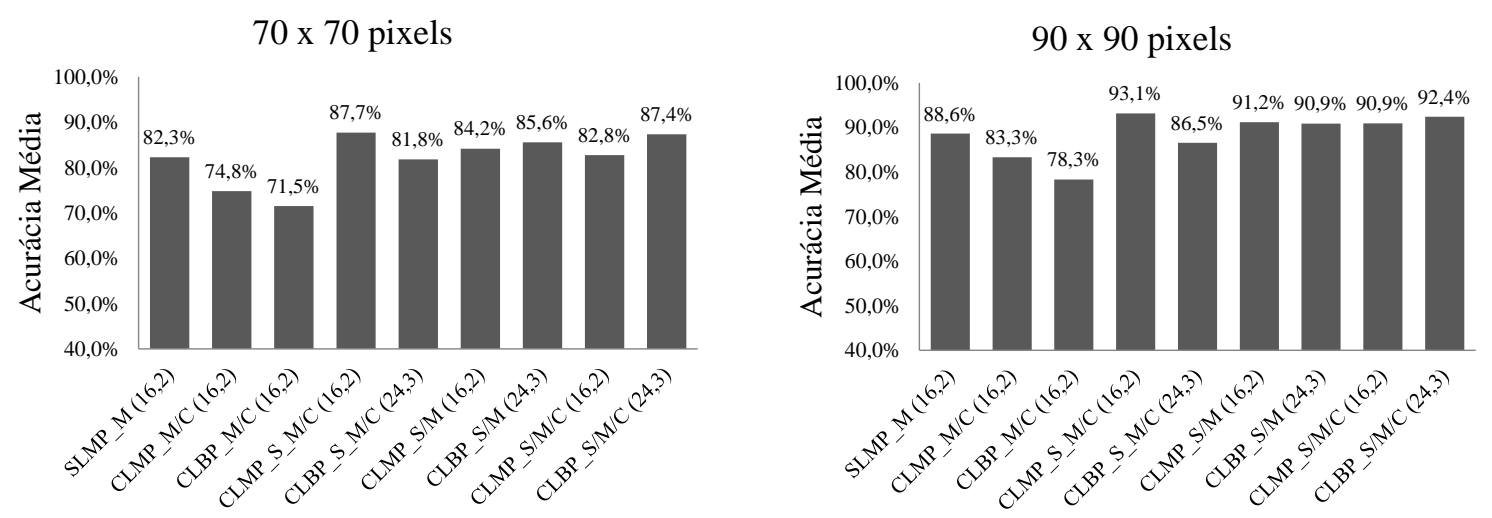

Figura 6.18 Acurácia média dos descritores com melhor desempenho em dois diferentes tamanhos de amostras rotacionadas pela interpolação Nearest Neighbor
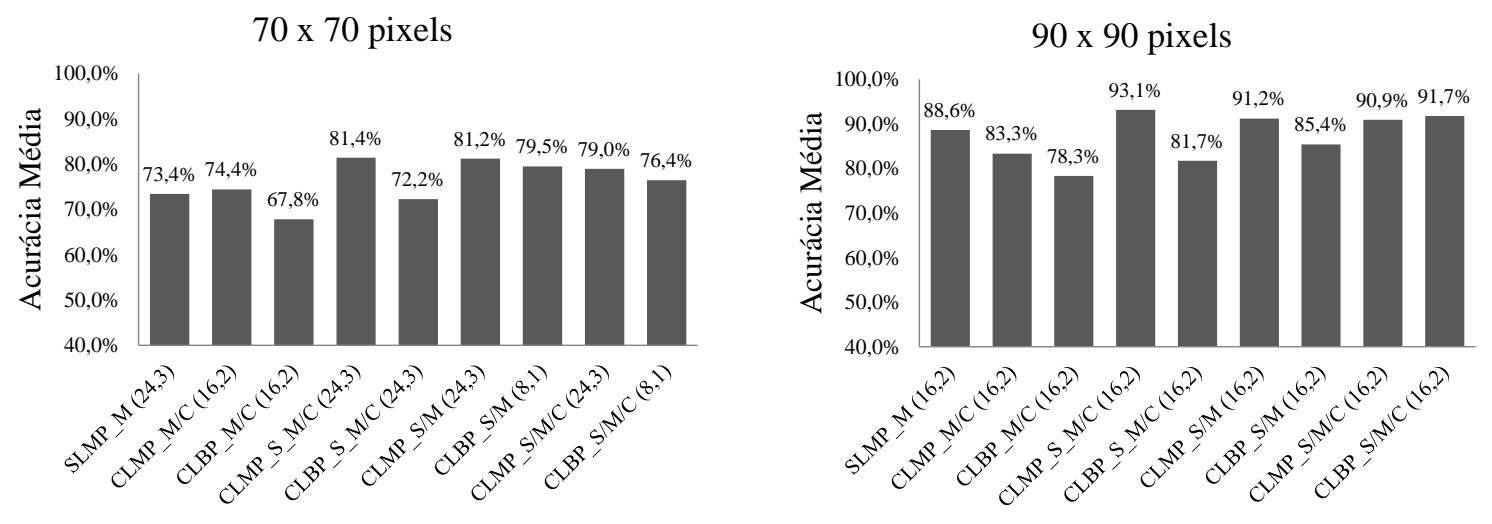

\subsection{Experimento 4}

O experimento 4 foi conduzido com as imagens da base Brodatz Texture Rotation da mesma maneira que foi realizado com a base de Kylberg e Sintorn, porém com menos amostras por classe, neste caso são avaliadas 50 amostras de tamanhos 70 x 70 pixels, 90 x 
90 pixels, 110 x 110 pixels, 130 x 130 pixels de cada classe analisada. Foram analisadas também diferentes configurações de raio e pontos de amostragem.

Para simplificação de análise, apenas os descritores de melhor desempenho foram apresentados graficamente, o resultados dos demais estão detalhados no Apêndice D. O gráfico da Figura 6.19 apresenta o desempenho do descritor SLMP_M para os diferentes métodos usados para rotação com os diferentes tamanhos de amostra, sendo que a configuração que alcançou melhor desempenho em todos os testes foi SLMP_M (24,3).

Figura 6.19 Acurácia média do descritor SLMP_M (24,3) para diferentes métodos de rotação e tamanho de amostra.

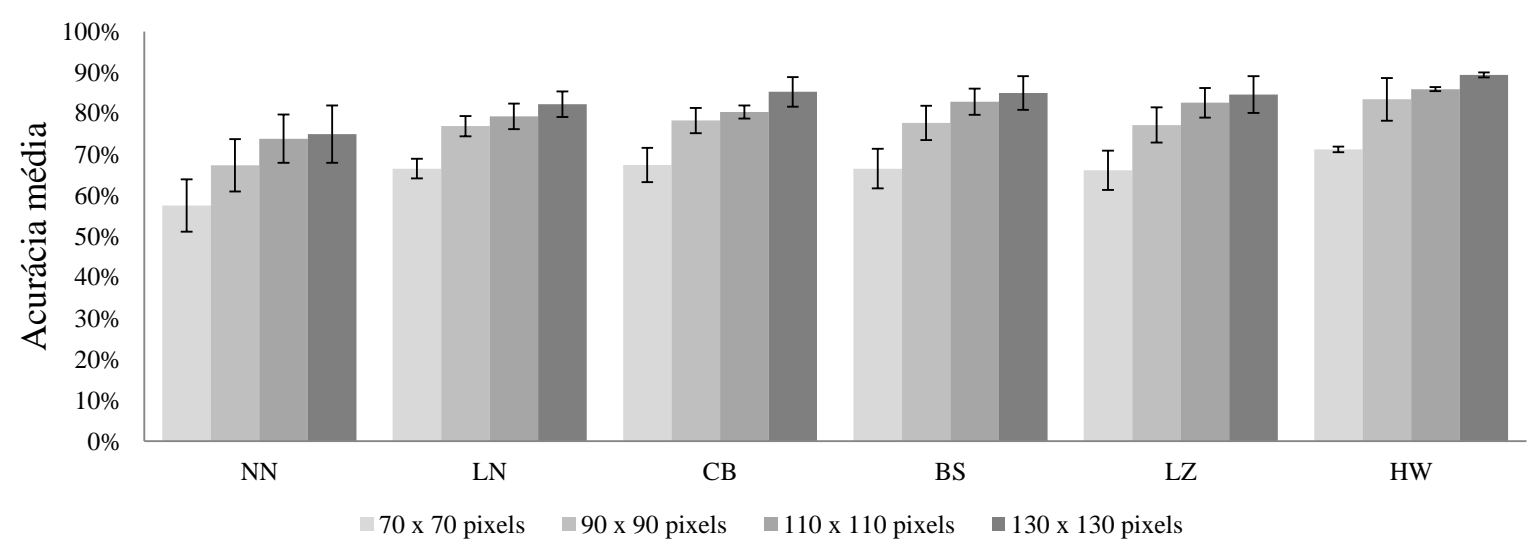

O gráfico da Figura 6.20 exibe os resultados para o melhor descritor CLMP, que neste experimento é o CLMP_S/M (24,3).

Figura 6.20 Acurácia média do descritor CLMP para diferentes métodos de rotação e tamanho de amostra.

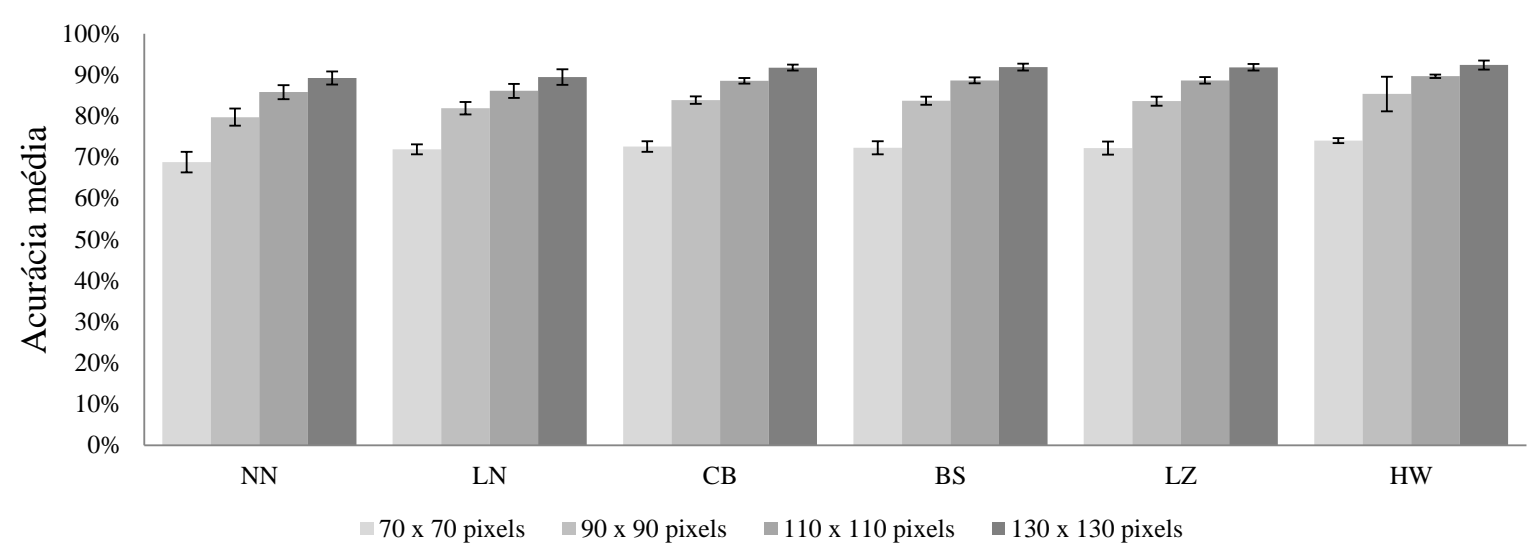

Diferentemente dos descritores propostos, neste teste com imagens da base de Brodatz, não há uma configuração única do descritor CLBP que alcance o melhor desempenho em todos os testes. Dependendo do método de interpolação ou do tamanho da 
amostra é necessário utilizar diferentes combinações dos operadores, configurações de raio e pontos de amostragem:

- Nearest Neighbor: para as amostras com 70 x 70 pixels o melhor resultado é obtido para a combinação CLBP_S_M/C (24,3), para os demais tamanhos, CLBP_S/M/C $(24,3)$;

- Linear: para todos os tamanhos de amostras a melhor combinação é CLBP_S/M/C $(24,3)$

- Cúbica: para as amostras com 130 x 130 pixels a melhor configuração é CLBP_S/M/C $(24,3)$ e, para o demais tamanhos de amostras, CLBP_S/M/C (16,2);

- B-spline: para as amostras com tamanho 70 x 70 pixels a melhor configuração é CLBP_S_M/C (16,2), para as amostras com 90 x 90 pixels é o CLBP_S/M/C $(24,3)$ e para os demais tamanhos de amostras a melhor combinação é CLBP_S/M/C (16,2);

- Lanczos: para todos os tamanhos de amostras a melhor combinação é CLBP_S/M/C $(16,2)$ e

- Hardware: para todos os tamanhos de amostras a melhor combinação é CLBP_S/M $(24,3)$.

O gráfico da Figura 6.21 exibe os resultados para as melhores configurações do descritor CLMP nos diferentes tamanhos de amostras e métodos de rotação.

Figura 6.21 Acurácia média do descritor CLBP para diferentes métodos de rotação e tamanho de amostra.

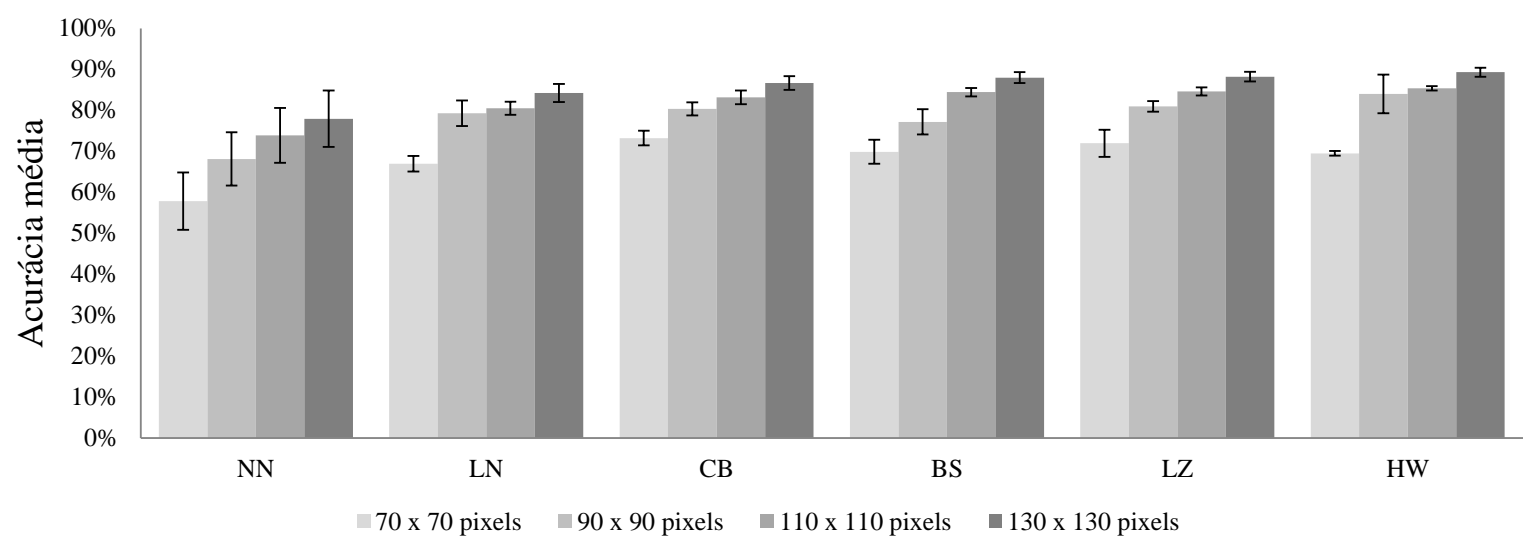

Assim como nos experimentos realizados com a base de Kylberg Sintorn, o aumento do tamanho da amostra aumenta a capacidade de descrição e consequentemente há uma melhora na sensibilidade mas, dependendo da aplicação é necessário fazer uma análise até quanto o custo para processar uma amostra maior não afeta a velocidade e desempenho do sistema como um todo. 
O melhor desempenho é observado com o descritor CLMP_S/M $(24,3)$ que obteve 92\% de acurácia média com amostras de tamanho 130 × 130 pixels ao rotacioná-las por hardware e pelas interpolações Cúbica, B-spline e Lanczos. Já o CLBP alcança seu melhor resultado com a configuração CLBP_S/M $(24,3)$ na rotação por hardware em amostras também com tamanho 130 x 130 pixels com 89\% de acurácia média da mesma forma que o descritor proposto SLMP_M (24,3).

Verifica-se que a acurácia média obtida pelos descritores CLMP em amostras com tamanho 110 x 110 pixels é ainda superior a acurácia média obtida pelos descritores CLBP com amostras de tamanho 130 x 130 pixels, ou seja, em amostras menores os descritores CLMP ainda apresentam maior capacidade de descrição que os descritores CLBP e isto é uma característica muito importante em aplicações online.

Em todos os experimentos realizados o descritor proposto SLMP_M não superou nenhuma das combinações dos operadores do CLMP, mostrando assim, que a combinação de operadores apenas via concatenação de vetores de características não é tão robusta quanto a combinação conjunta por meio do histograma 2-D.

\subsection{Experimento 5}

Além da robustez à rotação, o custo computacional é outra importante questão em aplicações no mundo real. A maioria dos sistemas de visão computacional é projetada para cenas reais e requerem processamento em tempo real. Assim, descritores de textura que produzem alta precisão com baixo custo computacional são desejáveis.

No experimento 5 é calculado o tempo computacional para geração do vetor de características para diferentes tamanhos de amostras de imagens da base com imagens do Álbum de Brodatz. Este experimento foi conduzido em um computador $2.5 \mathrm{GHz}$ Intel i73537U com 6GB de RAM e a programação foi realizada no MATLAB ${ }^{\circledR}$ release 2013a. A Tabela 6.5 exibe o tempo computacional médio em segundos (s) para geração dos vetores de características para os diferentes tamanhos de amostras analisados nos experimentos, para as diferentes configurações de raio e quantidade de pontos de amostragem e também para as diferentes combinações dos operadores dos descritores CLMP e CLBP.

Os diferentes descritores CLMP apesar de terem o mesmo tamanho dos vetores de características que os descritores CLBP, o tempo computacional é muito menor para geração dos vetores de características. Esta diferença é observada principalmente no caso com os vetores com maior dimensão, como é o caso dos descritores CLMP_S/M/C(24,3) e 
CLBP_S/M/C(24,3) que possuem dimensão de 1352 elementos e tempo computacional para geração do vetor de características em uma amostra de tamanho 110 x 110 pixels, de 0,114 s e $161,397 \mathrm{~s}$, respectivamente.

Nos experimentos realizados, a combinação de operadores do CLBP que apresentou melhor desempenho, de maneira geral, foi a CLBP_S/M/C. Está combinação é a que apresenta maior dimensionalidade e tempo de processamento para geração dos vetores de característica. Para o CLMP, a combinação de operadores que apresenta melhor desempenho é a CLMP_S/M, que possui um descritor bem menor e, consequentemente, demanda menos tempo para geração do vetor de características.

Tabela 6.5 Tempo computacional médio em segundos (s) para geração do vetor de características.

\begin{tabular}{|c|c|c|c|c|c|}
\hline Descritor & $\begin{array}{l}\text { Num. de } \\
\text { elementos }\end{array}$ & $\begin{array}{c}70 \times 70 \\
\text { pixels }\end{array}$ & $\begin{array}{c}90 \times 90 \\
\text { pixels }\end{array}$ & $\begin{array}{c}110 \times 110 \\
\text { pixels }\end{array}$ & $\begin{array}{c}130 \times 130 \\
\text { pixels }\end{array}$ \\
\hline SLMP_M $(8,1)$ & 148 & 0,035 & 0,047 & 0,048 & 0,055 \\
\hline SLMP_M $(16,2)$ & 227 & 0,038 & 0,535 & 0,067 & 0,080 \\
\hline SLMP_M $(24,3)$ & 228 & 0,040 & 0,066 & 0,082 & 0,098 \\
\hline CLMP_M/C $(8,1)$ & 20 & 0,047 & 0,059 & 0,065 & 0,080 \\
\hline CLMP_M/C $(16,2)$ & 36 & 0,050 & 0,068 & 0,079 & 0,094 \\
\hline CLMP_M/C $(24,3)$ & 52 & 0,051 & 0,082 & 0,096 & 0,114 \\
\hline CLMP_S_M/C $(8,1)$ & 30 & 0,069 & 0,081 & 0,087 & 0,100 \\
\hline CLMP_S_M/C $(16,2)$ & 54 & 0,071 & 0,091 & 0,102 & 0,120 \\
\hline CLMP_S_M/C $(24,3)$ & 78 & 0,072 & 0,100 & 0,118 & 0,138 \\
\hline CLMP_S/M $(8,1)$ & 100 & 0,048 & 0,059 & 0,064 & 0,076 \\
\hline CLMP_S/M $(16,2)$ & 324 & 0,055 & 0,076 & 0,084 & 0,099 \\
\hline CLMP_S/M $(24,3)$ & 676 & 0,053 & 0,079 & 0,096 & 0,119 \\
\hline CLMP_S/M/C $(8,1)$ & 200 & 0,053 & 0,060 & 0,066 & 0,075 \\
\hline CLMP_S/M/C $(16,2)$ & 648 & 0,051 & 0,069 & 0,082 & 0,100 \\
\hline CLMP_S/M/C $(24,3)$ & 1.352 & 0,053 & 0,080 & 0,099 & 0,115 \\
\hline CLBP_M/C $(8,1)$ & 20 & 0,056 & 0,063 & 0,068 & 0,075 \\
\hline CLBP_M/C $(16,2)$ & 36 & 0,679 & 0,695 & 0,699 & 0,724 \\
\hline CLBP_M/C $(24,3)$ & 52 & 160,243 & 162,600 & 162,487 & 163,144 \\
\hline CLBP_S_M/C $(8,1)$ & 30 & 0,082 & 0,089 & 0,090 & 0,101 \\
\hline CLBP_S_M/C $(16,2)$ & 54 & 0,701 & 0,710 & 0,718 & 0,735 \\
\hline CLBP_S_M/C $(24,3)$ & 78 & 161,247 & 161,560 & 160,653 & 162,346 \\
\hline CLBP_S/M $(8,1)$ & 100 & 0,056 & 0,064 & 0,069 & 0,075 \\
\hline CLBP_S/M $(16,2)$ & 324 & 0,672 & 0,679 & 0,694 & 0,719 \\
\hline CLBP_S/M $(24,3)$ & 676 & 161,310 & 161,583 & 160,960 & 163,512 \\
\hline CLBP_S/M/C $(8,1)$ & 200 & 0,056 & 0,631 & 0,670 & 0,076 \\
\hline CLBP_S/M/C $(16,2)$ & 648 & 0,687 & 0,687 & 0,695 & 0,709 \\
\hline CLBP_S/M/C $(24,3)$ & 1.352 & 160,393 & 160,605 & 160,341 & 161,399 \\
\hline
\end{tabular}




\subsection{Análise qualitativa}

Para realização de uma análise qualitativa dos resultados foram selecionadas matrizes de confusão geradas para cada descritor que obteve o melhor desempenho na base de Brodatz. O método de rotação que apresentou os melhores resultados foi a rotação por Hardware em que o descritor CLMP_S/M(24,3) obteve uma acurácia média de 92,32\%, o SLMP_M(24,3) 89,42\% e o CLBP_S/M(24,3) 89,30\%. Optou-se por observar as matrizes de confusão para os testes realizados com o ângulo de $60^{\circ}$, no qual os três descritores apresentaram um acurácia média dos 10-folds próxima a acurácia média ao longo de todos os ângulos, o CLMP_S/M(24,3) com 92,98\%,o SLMP_M(24,3) com 89,95\% e o CLBP_S/M $(24,3)$ com 90,34\%. As Figuras 6.22 a 6.24 mostram as matrizes de confusão para cada descritor avaliado. Na diagonal principal tem-se o verdadeiros positivos, ou seja, das 45 amostras de cada uma das 32 classes quantas foram classificadas corretamente. As colunas referem-se às classes de referência e as linhas às classes atribuídas.

Figura 6.22 Matriz de confusão do descritor CLMP_S/M $(23,4)$

\begin{tabular}{|c|c|c|c|c|c|c|c|c|c|c|c|c|c|c|c|c|c|c|c|c|c|c|c|c|c|c|c|c|c|c|c|c|}
\hline & & & & & & & & & & & & & & & & & & & & & & & & & C25] & C26] & $227 \sqrt{4}$ & C28 & $\mathrm{C} 2 \mathrm{C}^{\circ}$ & & & \\
\hline $\mathrm{Cl}$ & 45 & & 0 & 0 & 0 & & & 0 & 0 & 0 & & & & & & & & 0 & & $\begin{array}{ll} \\
\end{array}$ & & 0 & 0 & & & & & & & & & \\
\hline C2 & 0 & 15 & & 0 & 0 & 0 & 0 & 0 & 0 & 0 & 0 & 0 & 0 & 0 & 0 & 0 & 0 & 0 & 0 & 0 & 0 & 0 & 0 & 0 & 0 & 0 & 0 & 0 & 0 & & 0 & \\
\hline C3 & 0 & 0 & 45 & & 0 & 0 & 0 & & & 0 & 0 & 0 & 0 & 0 & 0 & 0 & & & 0 & 0 & 0 & 0 & 0 & 0 & \begin{tabular}{l|l}
0 \\
\end{tabular} & 0 & 0 & 0 & 0 & & 0 & \\
\hline $\mathrm{C} 4$ & 0 & 0 & 0 & 40 & & 0 & 6 & & & & 0 & 8 & 16 & & 0 & 0 & 0 & 0 & 0 & 0 & 0 & 0 & 0 & 0 & 2 & & & & & & & \\
\hline $\mathrm{C5}$ & 0 & 0 & 0 & & 45 & & 0 & 0 & 0 & 0 & 0 & 0 & 0 & 0 & 0 & 0 & 0 & 0 & 0 & 0 & 0 & 0 & 0 & 0 & $\begin{array}{ll}0 \\
\end{array}$ & 0 & 0 & 0 & 0 & 0 & 0 & \\
\hline C6 & \begin{tabular}{|l|l}
0 \\
\end{tabular} & 0 & 0 & 0 & 0 & 44 & 0 & 0 & 0 & 0 & 0 & 0 & 0 & 0 & 0 & 0 & 0 & 0 & 0 & 0 & 0 & 0 & 0 & 0 & \begin{tabular}{l|l}
0 \\
\end{tabular} & 0 & 0 & 0 & 0 & 0 & 0 & \\
\hline $\mathrm{C} 7$ & 0 & 0 & 0 & 0 & 0 & 1 & 39 & & 0 & 0 & 4 & 11 & 0 & 0 & 0 & 0 & 0 & 0 & 0 & 0 & 0 & 0 & 0 & 0 & 0 & 0 & 0 & 0 & & & 0 & \\
\hline $\mathrm{C} 8$ & 0 & 0 & 0 & 0 & 0 & 0 & & 45 & & 0 & 0 & 0 & 0 & 0 & 0 & 0 & 0 & 0 & 0 & 0 & 0 & 0 & $\begin{array}{ll} \\
\end{array}$ & & & & & & & & & \\
\hline C9 & 0 & 0 & 0 & 0 & 0 & 0 & 0 & & 45 & 0 & 0 & 0 & 0 & 0 & 0 & 0 & 0 & 0 & 0 & & 0 & 0 & & & & & & & & & & \\
\hline $\mathrm{C} 10$ & 0 & 0 & 0 & 0 & 0 & 0 & 0 & 0 & 0 & 42 & 0 & 0 & 0 & 0 & 1 & 0 & 0 & 0 & 0 & 0 & 0 & 0 & 0 & 0 & 0 & 0 & 0 & 0 & & & & \\
\hline C11 & 0 & 0 & 0 & 0 & 0 & 0 & 0 & 0 & 0 & 0 & 41 & 0 & 0 & 0 & 0 & 0 & 0 & 0 & 0 & 0 & 0 & 0 & 0 & & 0 & & 0 & & & & 0 & 0 \\
\hline $\mathrm{C} 12$ & 0 & 0 & 0 & 3 & 0 & 0 & 0 & 0 & 0 & 0 & 0 & 26 & 0 & 0 & 0 & 0 & 0 & 0 & 0 & 0 & 0 & 0 & 0 & 0 & 0 & 0 & & 0 & 0 & & 0 & \\
\hline $\mathrm{C} 13$ & 0 & 0 & 0 & 0 & 0 & 0 & 0 & 0 & 0 & 0 & 0 & 0 & 29 & 0 & 0 & 0 & 0 & 0 & 0 & 0 & 0 & 0 & 0 & 0 & 0 & 0 & 0 & & & & & \\
\hline C14 & 0 & 0 & 0 & 0 & 0 & 0 & 0 & 0 & 0 & 0 & 0 & 0 & 0 & 45 & 0 & 0 & 0 & 0 & 0 & 0 & 0 & 0 & 0 & 0 & 0 & 0 & 0 & 0 & 0 & 0 & 0 & \\
\hline C15 & 0 & 0 & 0 & 2 & 0 & 0 & 0 & 0 & 0 & 0 & 0 & 0 & 0 & 0 & 41 & 0 & 0 & 0 & 0 & 0 & 0 & 0 & 0 & 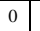 & 0 & & & & & & 0 & \\
\hline $\mathrm{C} 16$ & 0 & 0 & 0 & 0 & 0 & 0 & 0 & 0 & 0 & 0 & 0 & 0 & 0 & 0 & 0 & 40 & 0 & 0 & 0 & 0 & 0 & 0 & 0 & 0 & 0 & & & & & & & \\
\hline C17 & 0 & 0 & 0 & 0 & 0 & 0 & 0 & 0 & 0 & 0 & 0 & 0 & 0 & 0 & 0 & 0 & 43 & 0 & 0 & 0 & 0 & 0 & 0 & 0 & 0 & 0 & 0 & & & & 0 & \\
\hline C18 & 0 & 0 & 0 & 0 & 0 & 0 & 0 & 0 & 0 & 0 & 0 & 0 & 0 & 0 & 0 & 0 & 0 & 44 & 0 & 0 & \begin{tabular}{l|l}
0 \\
\end{tabular} & 0 & | 0 & | 0 & 0 & 0 & 0 & 0 & 0 & 0 & 0 & \\
\hline \begin{tabular}{|l|}
$\mathrm{C} 19$ \\
\end{tabular} & 0 & 0 & 0 & 0 & 0 & 0 & 0 & 0 & 0 & 0 & 0 & 0 & 0 & 0 & 0 & 0 & \begin{tabular}{l|l}
0 \\
\end{tabular} & 0 & 43 & 0 & 0 & 0 & \begin{tabular}{|l|}
0 \\
\end{tabular} & \begin{tabular}{|l|l|l} 
\\
\end{tabular} & 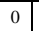 & 0 & 0 & 0 & 0 & 0 & 0 & \\
\hline C20 & 0 & 0 & 0 & 0 & 0 & 0 & 0 & 0 & 0 & 0 & 0 & 0 & 0 & 0 & 0 & 0 & 0 & 0 & 0 & 45 & 0 & 0 & 0 & 0 & 0 & 0 & & & & & & \\
\hline C21 & 0 & 0 & 0 & 0 & 0 & 0 & 0 & 0 & 0 & 0 & 0 & 0 & 0 & 0 & 0 & 0 & 1 & 0 & 0 & 0 & 44 & 0 & 0 & 0 & 0 & 0 & & & & & & \\
\hline $\mathrm{C} 22$ & 0 & 0 & 0 & 0 & 0 & 0 & 0 & 0 & 0 & 0 & 0 & 0 & 0 & 0 & 0 & 0 & 0 & 0 & 2 & \begin{tabular}{|l|} 
\\
\end{tabular} & 0 & 45 & 0 & 0 & 0 & 0 & 0 & 0 & \begin{tabular}{|l|l} 
\\
\end{tabular} & 0 & 0 & \\
\hline $\mathrm{C} 23$ & 0 & 0 & 0 & 0 & 0 & 0 & 0 & 0 & 0 & 0 & 0 & 0 & 0 & 0 & 0 & 0 & 0 & 0 & 0 & \begin{tabular}{|l|} 
\\
\end{tabular} & 0 & 0 & 42 & 0 & 0 & 0 & 0 & 0 & \begin{tabular}{|l|}
0 \\
\end{tabular} & 0 & 0 & 0 \\
\hline $\mathrm{C} 24$ & 0 & 0 & 0 & 0 & 0 & 0 & 0 & 0 & 0 & 0 & 0 & 0 & 0 & 0 & 0 & 0 & 0 & 0 & 0 & $\begin{array}{ll} \\
\end{array}$ & \begin{tabular}{l|l}
0 \\
\end{tabular} & 0 & 0 & 45 & 0 & 0 & 0 & 0 & \begin{tabular}{|l|}
0 \\
\end{tabular} & 0 & 0 & \\
\hline $\mathrm{C} 25$ & 0 & 0 & 0 & 0 & 0 & 0 & 0 & 0 & 0 & 0 & 0 & 0 & 0 & 0 & 0 & 0 & 0 & 0 & 0 & 0 & 0 & 0 & 0 & 0 & 42 & 0 & 0 & 0 & 0 & & 0 & \\
\hline $\mathrm{C} 26$ & 0 & 0 & 0 & 0 & 0 & 0 & 0 & 0 & 0 & 0 & 0 & 0 & 0 & 0 & 0 & 0 & 0 & 0 & 0 & 0 & 0 & 0 & 0 & 0 & 0 & 45 & & 0 & 0 & 0 & 0 & 0 \\
\hline C27 & 0 & 0 & 0 & 0 & 0 & 0 & 0 & 0 & 0 & 0 & 0 & 0 & 0 & 0 & 0 & 0 & 0 & 0 & 0 & 0 & 0 & 0 & 0 & | 0 & 0 & 0 & 43 & & 0 & 0 & 0 & 0 \\
\hline C28 & 0 & 0 & 0 & 0 & 0 & 0 & 0 & 0 & 0 & 3 & 0 & 0 & 0 & 0 & 0 & 5 & 0 & 0 & 0 & 0 & 0 & 0 & 3 & 0 & \begin{tabular}{ll|} 
\\
\end{tabular} & $\begin{array}{ll}0 \\
\end{array}$ & 2 & 40 & 0 & 2 & 0 & 0 \\
\hline \begin{tabular}{|l|}
$\mathrm{C} 29$ \\
\end{tabular} & 0 & 0 & 0 & 0 & 0 & 0 & 0 & 0 & 0 & 0 & 0 & 0 & 0 & 0 & 0 & 0 & 0 & 0 & 0 & 0 & 0 & 0 & 0 & 0 & \begin{tabular}{l|l}
0 \\
\end{tabular} & \begin{tabular}{l|l}
0 \\
\end{tabular} & 0 & & 45 & 0 & 0 & \\
\hline C30 & 0 & 0 & 0 & 0 & 0 & 0 & 0 & 0 & 0 & 0 & 0 & 0 & 0 & 0 & 3 & 0 & 0 & 0 & 0 & 0 & 0 & 0 & 0 & 0 & 0 & 0 & 0 & 0 & 0 & & 0 & \\
\hline $\mathrm{C} 31$ & 0 & 0 & 0 & 0 & 0 & 0 & 0 & 0 & 0 & 0 & 0 & 0 & 0 & 0 & 0 & 0 & 1 & 0 & 0 & 0 & 0 & 0 & 0 & 0 & 0 & 0 & 0 & 0 & 0 & & & \\
\hline $\mathrm{C} 2$ & & & & & & & & & & & & & & & & & & & & & & & & & & & & & & & & \\
\hline
\end{tabular}


Analisando-se os resultados da matriz de confusão da Figura 6.22, observa-se que o descritor CLMP_S/M (24,3) apresentou um excelente desempenho (definido como 45 acertos ou $100 \%$ de classificações corretas) em 14 classes, 44 acertos em 3 classes, 43 acertos em 3 classes, 42 acertos em 3 classes, 41 acertos em 2 classes, 40 acertos em 3 classes e, menos que 40 acertos em 4 classes. Dentre as classes que apresentaram menos acertos está a Classe 12, com 26 acertos apenas. Oito amostras desta classe foram confundidas com a Classe 4, e onze com as amostras da Classe 7. A Figura 6.23 mostra uma amostra da classe de referência e amostras das classes atribuídas para uma análise visual. Observa-se que há uma certa "semelhança visual" entre as classes, principalmente entre as classes C12 e C4, justificando assim, a confusão realizada no processo classificatório.

Figura 6.23 Exemplo de texturas classificadas incorretamente pelo descritor CLMP_S/M $(24,3)$

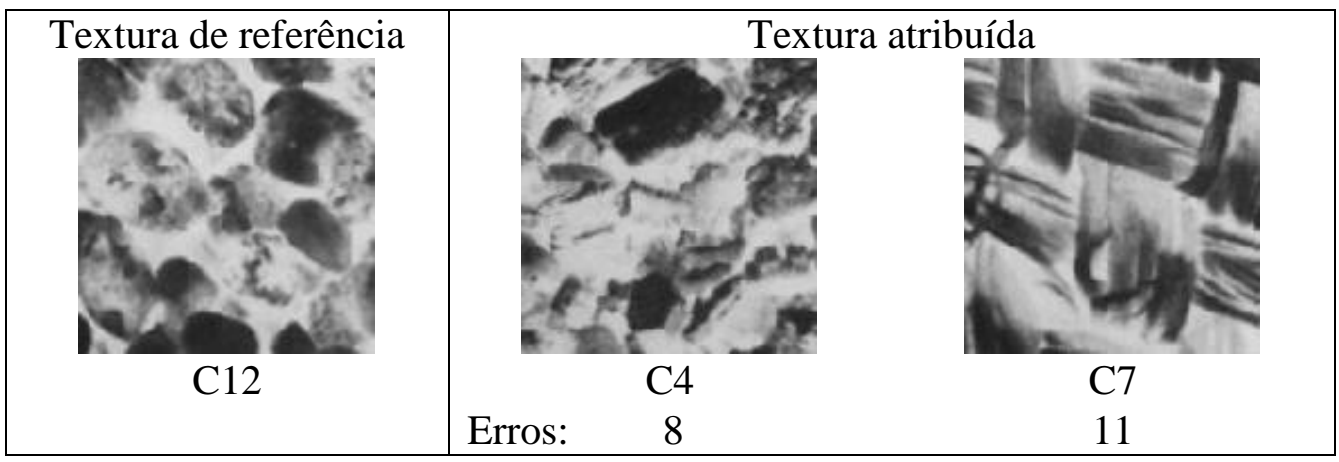

A Figura 6.24 exibe a matriz de confusão gerada ao aplicar o descritor SLMP_M $(24,3)$ no processo classificatório. Este descritor apresenta $100 \%$ de classificação correta em 15 classes, 44 acertos em 3 classes, 42 acertos em 4 classes, 41 acertos em 1 classe, 40 acertos em 1 classe e, menos que 40 acertos em 8 classes. 
Figura 6.24 Matriz de confusão do descritor SLMP_M (24,3)

\begin{tabular}{|c|c|c|c|c|c|c|c|c|c|c|c|c|c|c|c|c|c|c|c|c|c|c|c|c|c|c|c|c|c|c|c|c|}
\hline & $\mathrm{Cl}$ & C2 & C3 & C4 & C5 & C6 & C7 & C8 & C9 & & & & & & & & & & & & & & & & & & & & & & & \\
\hline & 45 & & 0 & 0 & & & & & & & 0 & 0 & 0 & 0 & 0 & 0 & & & 0 & 0 & & 0 & 0 & & & 0 & & & & & & \\
\hline C2 & & 27 & c & 0 & & & & & & & & & & & & & & & & & & & & & & & & & & & & \\
\hline & & & 4 & 0 & & & & & & & & & & & & & & & & & & & & & & & & & & & & \\
\hline & & & & & & & & & & & & & & & & & & & & & & & & & & & & & & & & \\
\hline & & & & & 41 & & & & & & & & & & & & & & & & & & & & & & & & & & & \\
\hline & & & & & & 36 & & & & & & & & & & & & & & & & & & & & & & & & & & \\
\hline & & & & & & & 44 & & & & & 18 & & & & & & & & & & & & & & & & & & & & \\
\hline & & & & & & & & & & & & & & & & & & & & & & & & & & & & & & & & \\
\hline & & & & & & & & & 45 & & & & & & & & & & & & & & & & & & & & & & & \\
\hline & & & ( & 0 & & & ( & & 0 & 34 & & ( & & 0 & & 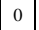 & & & & & & & & & & & & & & & & \\
\hline & & & & & & & & & & 0 & 45 & & & & & & & & & & & & & & & & & & & & & \\
\hline & 0 & & 1 & & & & & & & 0 & 0 & 25 & 0 & 0 & 1 & ( & & & & & & & & & & & & & 0 & & & \\
\hline 13 & & & & & & & & & & & & & & & & ( & & & & & & & & & & & & & & & & \\
\hline 14 & 0 & & & & & & & & & & 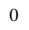 & 0 & 0 & 45 & & 0 & & & & & & & & & & 0 & & & & & 0 & 0 \\
\hline 15 & & & & & & & & & & & & & & & 42 & 0 & & & & & & & & & & & & & & & & \\
\hline 16 & 0 & & & & & & & & & & & 0 & & & 0 & 45 & & & & 0 & & & & & & f & & & 0 & & 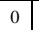 & 0 \\
\hline 17 & & & & & & & & & & & & & & & & 0 & 42 & & & & & & & & & & & & & & & \\
\hline 18 & & & & & & & & & & & & & & & & 0 & 0 & 45 & & 0 & 0 & & & 0 & J & 0 & 0 & & & 0 & 0 & 0 \\
\hline & & & & & & & & & & & & & & & & & & & 45 & & & & & & & & & & 14 & & & \\
\hline 20 & 0 & & & & & & & & & & & & & & & 0 & & & 0 & 45 & & & & 0 & & & 0 & & & & 0 & 0 \\
\hline 21 & & & & & & & & & & & & & & & & & & & & 0 & & & & & & & & & & & & \\
\hline 22 & 0 & & & & & & & & & & & ( & & & & 0 & & & 0 & 0 & 0 & 42 & 0 & 0 & & 0 & 0 & & 0 & 0 & 0 & 0 \\
\hline & 0 & & & & & & & & & & & & & & & & & & & & & 0 & 45 & & & & & & & & & \\
\hline 24 & 0 & 0 & 0 & 0 & & 0 & ( & & & & ( & 0 & 0 & 0 & & 0 & 0 & & ( & 0 & & 0 & 0 & 45 & & 0 & 0 & C & 0 & 0 & 0 & 0 \\
\hline 25 & 0 & & & & & & & & & & & & & & & & & & & & & & & 0 & 45 & & & & & & & \\
\hline 26 & 0 & 0 & 0 & 0 & & ( & 0 & & & 0 & 0 & 0 & 0 & 0 & & 0 & 0 & & 0 & 0 & 0 & 0 & 0 & 0 & 0 & 45 & 0 & 0 & 0 & 0 & 0 & 0 \\
\hline & 0 & & & & & & & & & & & & & & & & & & & & & & & 0 & & 0 & 44 & & & 0 & & 0 \\
\hline 28 & 0 & 0 & 0 & 0 & & 0 & 0 & C & 0 & ( & 0 & 0 & 0 & 0 & ( & 0 & 0 & & 0 & 0 & 0 & 0 & 0 & 0 & 0 & 0 & 1 & 45 & 0 & 0 & 0 & 0 \\
\hline 29 & 0 & & c & 6 & & & & & & & & & 0 & 0 & & 0 & 0 & & & 0 & & & & 0 & & o & c & 0 & 28 & 0 & & 0 \\
\hline 30 & 0 & 0 & 0 & 0 & 0 & 0 & 0 & 0 & 0 & 0 & 0 & 0 & 0 & 0 & 0 & 0 & 0 & 0 & 0 & 0 & 0 & 0 & 0 & 0 & 0 & 0 & 0 & 0 & 0 & 27 & 0 & 0 \\
\hline 31 & 0 & & 3 & 0 & & 0 & 0 & & & & & 0 & 0 & 0 & & 0 & 3 & & 0 & 0 & $\sigma$ & & 0 & 0 & & 0 & 0 & 0 & 0 & & & \\
\hline & 0 & & & 0 & & & & & & & 0 & 0 & 0 & 0 & & 0 & 0 & & & & & & 0 & 0 & & & 0 & 0 & & & & \\
\hline
\end{tabular}

Para o descritor SLMP_M $(24,3)$ a classe que apresentou menos acertos foi a Classe 13, com 10 acertos. As 35 demais amostras foram confundidadas com as amostras de imagens de textura de uma única classe, a classe C4. A Figura 6.25 exibe uma amostra da textura de referência da Classe 13 e uma amostra da Classe 4 confundida. Diferentemente do que aconteceu com as texturas atribuídas na Figura 6.23, não há neste caso, uma "semelhança visual" entre as texturas.

Figura 6.25 Exemplo de texturas classificadas incorretamente pelo descritor SLMP_M $(24,3)$

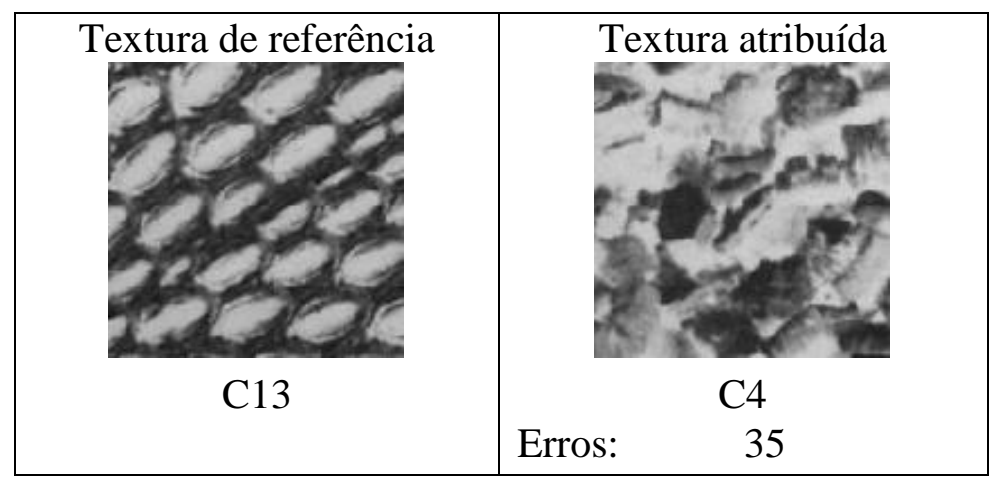

A matriz de confusão para o descritor CLBP_S/M $(24,3)$ é exibida na Figura 6.26. 
Figura 6. 26 Matriz de confusão do descritor CLBP_S/M $(24,3)$

\begin{tabular}{|c|c|c|c|c|c|c|c|c|c|c|c|c|c|c|c|c|c|c|c|c|c|c|c|c|c|c|c|c|c|c|c|}
\hline & & & & & & & & & & & & & & & & & & & & & & & & & & & & 3 & $\mathrm{C} 30$ & C31C & \\
\hline $\mathrm{Cl}$ & & & 0 & \begin{tabular}{l|l}
0 \\
\end{tabular} & 0 & \begin{tabular}{l|l} 
\\
\end{tabular} & 0 & \begin{tabular}{l|l}
0 \\
\end{tabular} & 0 & & & & & & & & & & & & & & & & & 0 & & \begin{tabular}{|l|l}
0 \\
\end{tabular} & \begin{tabular}{|l|l|}
0 \\
\end{tabular} & 0 & \\
\hline C2 & & 45 & & 0 & 18 & 0 & 0 & 0 & 0 & 0 & 0 & 0 & 0 & 0 & 0 & 0 & 0 & 0 & 0 & 0 & 0 & 0 & 0 & 0 & 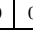 & 0 & 0 & 0 & 0 & 0 & 0 \\
\hline C3 & 0 & 0 & 45 & & 0 & 0 & 0 & 0 & 0 & \begin{tabular}{l|l} 
\\
\end{tabular} & 0 & 0 & 0 & 0 & 0 & 0 & 0 & 0 & 0 & 0 & 0 & & & & & 0 & & 0 & & 0 & \\
\hline $\mathrm{C} 4$ & & 0 & 0 & 32 & & 0 & 0 & 0 & 0 & & & & & & 0 & 0 & 0 & 0 & 0 & & & & & & & & & 0 & 0 & 0 & \\
\hline C5 & & 0 & 0 & 0 & 27 & 0 & 0 & 0 & 0 & 0 & & & & & & & & & & & & & & 0 & & 0 & 0 & 0 & 0 & 0 & 0 \\
\hline C6 & & 0 & 0 & 0 & 0 & 35 & 0 & 0 & 0 & 0 & 0 & 1 & 0 & 0 & 9 & 0 & 0 & 0 & 0 & 0 & & 0 & 0 & 0 & & 0 & 0 & 0 & 0 & 0 & 0 \\
\hline \begin{tabular}{|l|}
$\mathrm{C} 7$ \\
\end{tabular} & 0 & 0 & 0 & 0 & 0 & 0 & 40 & 0 & 0 & 0 & 0 & 0 & 0 & 0 & 0 & 0 & 0 & 0 & 0 & 0 & 0 & 0 & 0 & 0 & & 1 & 0 & 0 & 0 & 0 & 0 \\
\hline $\mathrm{C} 8$ & & 0 & 0 & 0 & 0 & 0 & 0 & 45 & & 0 & 0 & 0 & 0 & 0 & 0 & 0 & 0 & 0 & 0 & 0 & 0 & & & & & 0 & 0 & 0 & & 0 & 0 \\
\hline \begin{tabular}{|l|} 
C9 \\
\end{tabular} & 0 & 0 & 0 & 0 & 0 & 0 & 0 & & 45 & 0 & 0 & 0 & 0 & 0 & 0 & 0 & 0 & 0 & 0 & 0 & 0 & & & & & 0 & 0 & 0 & & 0 & \\
\hline \begin{tabular}{|l|}
$\mathrm{C} 10$ \\
\end{tabular} & & 0 & 0 & 0 & 0 & 0 & 0 & 0 & 0 & 22 & 0 & 0 & 2 & 0 & 0 & 0 & 0 & 0 & 0 & 0 & 0 & 0 & 0 & 0 & & 3 & 0 & 0 & 0 & 0 & 0 \\
\hline C11 & & 0 & 0 & 0 & 0 & 0 & 0 & 0 & 0 & 0 & 44 & 0 & 0 & 0 & 0 & 0 & 0 & 0 & 0 & 0 & 0 & 0 & 0 & 0 & & 0 & 0 & & 4 & 0 & 0 \\
\hline $\mathrm{Cl} 2$ & & 0 & 0 & 7 & 0 & 10 & 3 & 0 & 0 & 0 & 0 & 39 & 0 & 0 & 1 & 0 & 0 & 0 & 0 & 0 & 0 & 0 & & & & 1 & 0 & 0 & 0 & 0 & 0 \\
\hline \begin{tabular}{|l|}
$\mathrm{C} 13$ \\
\end{tabular} & & 0 & 0 & 0 & 0 & 0 & 0 & 0 & 0 & 6 & 0 & 0 & 43 & 0 & 0 & 0 & 0 & 0 & 0 & 0 & & & & & & 0 & 0 & 0 & & 0 & 0 \\
\hline $\mathrm{C} 14$ & & 0 & 0 & 0 & 0 & 0 & 0 & 0 & 0 & 0 & 0 & 0 & 0 & 45 & 0 & 0 & 0 & 0 & 0 & 0 & 0 & 0 & 0 & 0 & ( & 0 & 0 & 0 & 0 & 0 & 0 \\
\hline $\mathrm{C} 15$ & & 0 & 0 & 0 & 0 & 0 & 0 & 0 & 0 & 10 & 0 & 0 & 0 & 0 & 35 & 0 & 0 & 0 & 0 & 0 & 0 & 0 & 0 & 0 & & 0 & 0 & 0 & 0 & 0 & 0 \\
\hline $\mathrm{C} 16$ & & 0 & 0 & 0 & 0 & 0 & 0 & 0 & 0 & 0 & 0 & 0 & 0 & 0 & & 45 & & 0 & 0 & 0 & 0 & 0 & 0 & 0 & . & 0 & 0 & & & 0 & \\
\hline C17 & & 0 & 0 & 0 & 0 & 0 & 0 & 0 & 0 & 0 & 0 & 0 & 0 & 0 & 0 & 0 & 45 & 0 & 0 & 0 & 0 & & & 0 & & 0 & 0 & 0 & 0 & 0 & 0 \\
\hline C18 & 0 & 0 & 0 & 0 & 0 & 0 & 0 & 0 & 0 & 0 & 0 & 0 & 0 & 0 & 0 & 0 & 0 & 45 & 0 & 0 & 0 & 0 & 0 & 0 & & 0 & 0 & 0 & 0 & 0 & 0 \\
\hline \begin{tabular}{|l|} 
C19 \\
\end{tabular} & & 0 & 0 & 0 & 0 & 0 & 0 & 0 & 0 & 0 & 0 & 0 & 0 & 0 & 0 & 0 & 0 & 0 & 45 & 0 & 0 & & 0 & 0 & & 0 & 0 & 6 & 0 & 0 & 0 \\
\hline C20 & & 0 & 0 & 0 & 0 & 0 & 0 & 0 & 0 & 0 & 0 & 0 & 0 & 0 & 0 & 0 & 0 & 0 & 0 & 45 & 0 & 0 & 0 & 0 & . & 0 & 0 & 0 & 0 & 0 & 0 \\
\hline C21 & 0 & 0 & 0 & 0 & 0 & 0 & 0 & 0 & 0 & 0 & 0 & 0 & 0 & 0 & 0 & 0 & 0 & 0 & 0 & 0 & 42 & 0 & 0 & 0 & & 0 & 0 & 0 & 0 & 0 & 0 \\
\hline \begin{tabular}{l|}
$\mathrm{C} 22$ \\
\end{tabular} & & 0 & 0 & 0 & 0 & 0 & 0 & 0 & 0 & 0 & 0 & 0 & 0 & 0 & 0 & 0 & 0 & 0 & 0 & 0 & 0 & 44 & 0 & 0 & & 0 & 0 & 0 & 0 & 0 & 0 \\
\hline \begin{tabular}{|l|}
$\mathrm{C}$ \\
\end{tabular} & 0 & 0 & 0 & 0 & 0 & 0 & 0 & 0 & 0 & 0 & 0 & 0 & 0 & 0 & 0 & 0 & 0 & 0 & 0 & 0 & 0 & 0 & 38 & 0 & t & 0 & 0 & 0 & 0 & 0 & 0 \\
\hline C24 & & 0 & 0 & 0 & 0 & 0 & 0 & 0 & 0 & 0 & 0 & 0 & 0 & 0 & 0 & 0 & 0 & 0 & 0 & 0 & 0 & 0 & 0 & 45 & 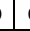 & 0 & 0 & 0 & 0 & 0 & 0 \\
\hline C25 & & 0 & 0 & 0 & 0 & 0 & 0 & 0 & 0 & 0 & 0 & 0 & 0 & 0 & 0 & 0 & 0 & 0 & 0 & 0 & 0 & 0 & 0 & \begin{tabular}{l|l}
0 & 4
\end{tabular} & ) & 0 & 0 & 0 & 0 & 0 & 0 \\
\hline C26 & & 0 & 0 & 0 & 0 & 0 & 0 & 0 & 0 & 0 & 0 & 0 & 0 & 0 & 0 & 0 & 0 & 0 & 0 & 0 & 0 & 0 & 0 & 0 & 4 & 50 & 0 & 0 & 0 & 0 & 0 \\
\hline C27 & & 0 & 0 & 0 & 0 & 0 & 2 & 0 & 0 & 0 & 0 & 0 & 0 & 0 & 0 & 0 & 0 & 0 & 0 & 0 & 0 & 0 & 5 & 0 & 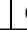 & 28 & 0 & 0 & 0 & 0 & 0 \\
\hline \begin{tabular}{|l|} 
C28 \\
\end{tabular} & & 0 & 0 & 6 & 0 & 0 & 0 & 0 & 0 & 0 & 0 & 0 & 0 & 0 & 0 & 0 & 0 & 0 & 0 & 0 & 0 & 0 & 0 & 0 & . & 5 & 45 & 0 & 0 & 0 & 0 \\
\hline \begin{tabular}{|l|} 
C29 \\
\end{tabular} & & 0 & 0 & 0 & 0 & 0 & 0 & 0 & 0 & 0 & 0 & 0 & 0 & 0 & 0 & 0 & 0 & 0 & 0 & 0 & 0 & 0 & 0 & 0 & t & 0 & 0 & 39 & 0 & 0 & 0 \\
\hline C30 & & 0 & 0 & 0 & 0 & 0 & 0 & 0 & 0 & 7 & 1 & 0 & 0 & 0 & 0 & 0 & 0 & 0 & 0 & 0 & 0 & 0 & 2 & 0 & & 0 & 0 & 0 & 33 & 0 & 0 \\
\hline C31 & & 0 & 0 & 0 & 0 & 0 & 0 & 0 & 0 & 0 & 0 & 0 & 0 & 0 & 0 & 0 & 0 & 0 & 0 & 0 & 0 & 0 & 0 & 0 & & 0 & 0 & 0 & & 45 & \\
\hline C32 & & & & & & & & & & & & & & & & & & & & & & & & & & & & & & & 45 \\
\hline
\end{tabular}

Este descritor apresenta o melhor desempenho em 16 classes, 44 acertos em 2 classes, 43 acertos em 1 classe, 42 acertos em 1 classe, 40 acertos em 2 classes e, menos que 40 acertos em 10 classes. O pior caso de classificação é observado na Classe 10, com 22 acertos. Esta classe é confundida com as Classes 13, 15 e 30. A Figura 6.27 mostra amostras das classes atribuídas e a quantidades de amostras classificadas erroneamente e observa-se também que, as amostras das classes atribuídas diferem, e muito, da textura da classe de referência.

Figura 6.27 Exemplo de texturas classificadas incorretamente pelo descritor CLBP_S/M $(24,3)$

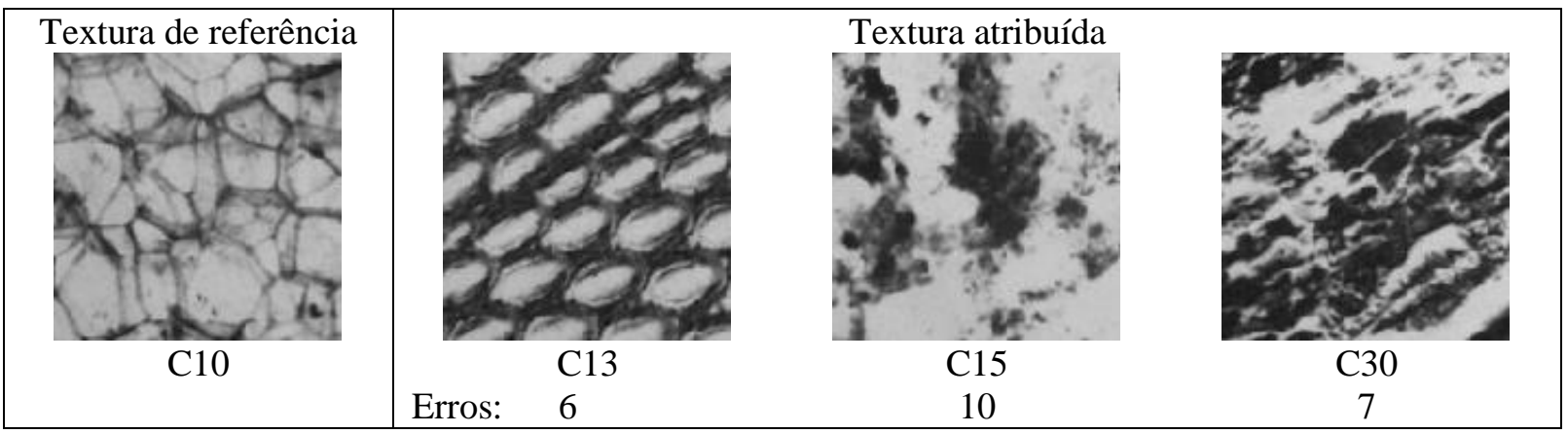


Apesar dos descritores SLMP_M (24,3) e CLBP_S/M (24,3) apresentarem mais classificações $100 \%$ corretas, 15 e 16, respectivamente, o descritor CLMP_S/M (24,3) em 28 classes apresentou 40 ou mais acertos, enquanto que o descritor SLMP_M $(24,3)$, apresentou 40 ou mais acertos em 24 classes e o CLBP_S/M $(24,3)$ em 22 classes.

\subsection{Considerações finais}

Neste capítulo foram apresentados de maneira resumida os resultados de experimentos conduzidos com duas bases de imagens de texturas rotacionadas ao se aplicar diferentes descritores de textura. Foram explorados diferentes configurações para os experimentos realizados: variedade de tamanhos de amostras e ângulos de rotação, quantidade de classes, combinação dos operadores dos descritores, tamanho de raio e pontos de amostragem dos descritores. E por fim, uma análise qualitativa foi realizada para avaliar o comportamento dos descritores no processo classificatório das imagens de textura. 


\section{Capítulo 7}

\section{Conclusões}

Em muitas aplicações de análise de textura é necessário o uso de técnicas invariantes ou robustas a rotações da imagem de entrada. Neste sentido, esta tese propôs descritores que diante dos testes realizados se mostraram robustos à variação de rotação quando comparados com descritores estado da arte, entre eles o CLBP que vem sendo aplicado com sucesso em vários trabalhos.

Foi mostrado que uma pequena variação na imagem pode alterar o bit de "0" para "1" e vice versa na codificação realizada pelos descritores baseados no LBP. Como os descritores propostos são uma evolução do descritor paramétrico LMP, a suavização proveniente da função de mapeamento sigmoidal permite uma maior robustez às variações nas imagens no processo de codificação dos micropadrões.

Outra característica dos descritores baseados no método LMP é a sua formulação paramétrica que permite diferentes possibilidades. Assim, uma das contribuições deste trabalho foi o desenvolvimento de um algoritmo de otimização por enxame de partículas para otimização conjunta da inclinação da curva sigmoide $(\beta)$ e a quantidade de bins $(B)$ realizada anteriormente de maneira empírica. Desta maneira é possível encontrar parâmetros ótimos para diferentes conjuntos de imagens, dependendo da aplicação realizada.

Descritores de micropadrões assumem que as texturas têm duas características complementares localmente: estrutura espacial (padrões) e contraste (intensidade dos padrões). Na formulação básica dos descritores LMP e LBP a informação da magnitude das diferenças dos níveis de cinza do micropadrão não é analisada. Assim, a inserção do operador que codifica a magnitude de um micropadrão também nas evoluções do descritor LMP, propostas neste trabalho, acarreta em uma melhor descrição das imagens de textura.

Também é demonstrado que a fusão de diferentes operadores melhora a classificação, e a maneira de combiná-los também influencia no resultado a exemplo dos descritores CLMP_S/M e SLMP_M; no primeiro, o vetor de características é gerado a partir de um histograma conjunto 2-D dos operadores CLMP_S e CLMP_M, já o vetor de características do segundo, é gerado ao se concatenar o descritor SLMP com a função que mapeia o sinal do micropadrão com o descritor SLMP com a função que mapeia a magnitude do micropadrão. A 
combinação por concatenação não supera a combinação conjunta dos vetores de características gerados pelos operadores.

Um descritor para ser considerado invariante à rotação deve mostrar variações sutis sobre diferentes orientações de uma textura independentemente do ângulo da textura. Isto é, um descritor que apresente um excelente desempenho para um determinado ângulo de rotação da imagem e decai para outros é extremamente dependente de conhecimento prévio sobre os ângulos avaliados, o que não é uma condição normal para imagens reais. No experimento $1 \mathrm{o}$ desvio padrão dos descritores que combinam característica de Fourier e variações do LBP é alto quando comparado com os descritores propostos. Isto demonstra que há uma grande variação de acurácia ao longo de diferentes ângulos, e em aplicações reais que as imagens se apresentam com ângulos arbitrários, esta não é uma boa característica.

Para todos os casos analisados para as imagens da base do Álbum de Brodatz, isto é, rotações, tamanho de amostras e métodos de rotação, o descritor proposto CLMP com a configuração CLMP_S/M $(3,24)$ apresentou o melhor desempenho. Isto não acontece com o descritor CLBP. Dependendo da situação analisada diferentes combinações podem apresentar um melhor desempenho, ou seja, o desempenho do CLBP é dependente das configurações avaliadas e não há uma combinação de operadores "universal" para descrever as imagens da base analisada.

Para os experimentos realizados com as imagens da base de Kylberg Sintorn a configuração do descritor CLMP que apresentou melhores resultados foi a CLMP_S_M/C $(16,2)$. Apenas para amostras com tamanho 120 x 120 pixels, para as imagens com rotações realizadas por Hardware e pelas interpolações Lanczos e Nearest Neighbor que a configuração CLMP_S/M/C apresentou melhor desempenho. Já para o descritor CLBP a configuração que apresentou, de maneira geral, o melhor desempenho foi a CLBP_S/M/C, porém, dependendo do tamanho de amostra e método de rotação avaliado, é necessário o uso de diferentes tamanhos de raio e quantidade de pontos de amostragem.

No que se refere à escolha de métodos de interpolação, as que obtiveram melhor desempenho foram Lanczos e B-spline, superando inclusive, rotações realizadas por Hardware em alguns casos. Isto demonstra que a rotação computacional com algoritmos de maior desempenho traz ganhos para a classificação de texturas rotacionadas. Apesar das interpolações Linear e Cúbica não se mostrarem adequadas na manutenção de características das texturas, ainda são as mais comumente utilizadas por softwares de processamento de imagem, como é o caso do MATLAB®, que utiliza em seu processamento interpolação Linear, Cúbica e a nearest neighbor. 
Nas aplicações de visão computacional que avaliem se uma determinada imagem de textura é similar a alguma outra previamente armazenada em um banco de dados, pode ser impossível determinar qual é o ângulo que a mesma foi obtida. Este é um exemplo de imagens obtidas por drones em agricultura de precisão. Desta maneira, a imagem adquirida pode ser comparada diretamente com a base, normalmente armazenada em ângulo $\theta=0^{\circ}$ ou rotacionada por um algoritmo eficiente como os que utilizam as interpolações Laczos ou $B$ spline, tanto em acurácia como em tempo de processamento.

Para ser considerado um bom descritor de textura, a técnica deve atingir dois objetivos concorrentes: baixa complexidade computacional, para permitir que a tarefa de classificação seja executada em tempo real; e, capturar a informação de textura mais representativa de uma classe, de modo que diferentes classes de texturas possam ser distinguidas apesar da presença de várias distorções de imagem como, por exemplo, a rotação, que foi investigada neste trabalho. No experimento 5, para medição do tempo computacional para geração dos vetores de características, os descritores baseados no método LMP demandam menos tempo de processamento, mesmo nos casos em que os vetores têm a mesma dimensão dos apresentados pelo CLBP.

Os resultados de acurácia obtidos nas análises com as imagens da base rotacionada do Álbum de Brodatz foram inferiores aos obtidos na base de Kylberg. Isto é justificado pela variedade de ângulos analisados, 21 ao invés de 9 e, maior quantidade de classes avaliadas, 32 ao invés de 25. Outra justificativa refere-se à qualidade das imagens, pois, durante o processo de aquisição, a imagens foram salvas em formato JPG, ou seja, sofreram compressão. Além disso, não houve nenhum controle da iluminação das texturas, enquanto estavam sendo rotacionadas.

Com isso, conclui-se que os objetivos propostos foram atingidos. Os descritores propostos incluindo as informações de magnitude e sinal mostraram-se mais robustos que outros comparados na classificação de texturas rotacionadas tanto por hardware como por software e que os melhores desempenhos ocorreram quando o método de interpolação é do tipo Lanczos o B-spline. O algoritmo de otimização desenvolvido e baseado em PSO gerou valores otimizados de $(\beta)$ e quantidade de $\operatorname{bins}(B)$ facilitando o uso, não somente para este trabalho, mas também para qualquer outro novo desenvolvimento ou aplicação da abordagem LMP. Um novo banco de texturas rotacionadas foi gerado e está disponível para toda a comunidade científica em http://imagem.sel.eesc.usp.br/base. 


\subsection{Sugestões para Trabalhos Futuros}

A partir da investigação realizada, novas propostas surgem visando trabalhos futuros:

- Neste trabalho é demonstrado que a combinação de descritores resulta em vetores de características com informações mais discriminantes. Outra análise que pode ser realizada é a combinação de descritores com multiresoluções, pois, neste trabalho foram combinados descritores com mesma configuração de raio e pontos de amostragem.

- Digitalização das demais classes do Álbum de Brodatz impressas, inclusive com iluminação controlada.

- Associar os descritores de textura propostos a Redes Neurais Convolutivas em problemas de classificação de textura, acompanhando a tendência recente, a exemplo dos trabalhos de Marcos, Volpi e Tuia (2016).

\subsection{Publicações oriundas do trabalho}

Trabalhos completos publicados em anais de congressos

VIEIRA, Raissa Tavares; GONZAGA, Adilson. Evaluation of robustness against interpolation methods of texture descriptors in image classification. In: XII Workshop de Visão Computacional, Campo Grande, 2016.

SOUZA, Jones Mendonça; VIEIRA, Raissa Tavares; GONZAGA, Adilson . Analysis of texture under Pupil Contraction/Dilation for Biometric Recognition. In: XI Workshop de Visão Computacional, São Carlos, 2015.

VIEIRA, Raissa Tavares; LANGONI, Virgílio de Melo; GONZAGA, Adilson. Videobased Iris Recognition by Quasi-Dynamic Texture Analysis. In: X Workshop de Visão Computacional, Uberlândia, 2014.

Capítulo de livro

VIEIRA, Raissa Tavares; NEGRI, Tamiris Trevisan; GONZAGA, Adilson. Robustness of Rotation Invariant Descriptors for Texture Classification. In: International Symposium on Visual Computing. Springer International Publishing, 2016. p. 268-277. 


\section{Referências}

ABDULLAHI, Muhammad Bashir; IDRIS, Fati; MOHAMMED, Adamu Alhaji. Performance analysis of particle swarm optimization algorithm-based parameter tuning for fingerprint image enhancement. In: Future Technologies Conference (FTC). IEEE, 2016. p. 528-536.

AHONEN, Timo et al. Rotation invariant image description with local binary pattern histogram fourier features. Image analysis, p. 61-70, 2009.

AHONEN, Timo; HADID, Abdenour; PIETIKAINEN, Matti. Face description with local binary patterns: Application to face recognition. IEEE transactions on pattern analysis and machine intelligence, v. 28, n. 12, p. 2037-2041, 2006.

BIANCONI, Francesco et al. Automatic classification of granite tiles through colour and texture features. Expert Systems with Applications, v. 39, n. 12, p. 11212-11218, 2012.

BIGUN, Josef; GRANLUND, Goesta H.. ; WIKLUND, Johan. Multidimensional orientation estimation with applications to texture analysis and optical flow. IEEE Transactions on Pattern Analysis and Machine Intelligence, v. 13, n. 8, p. 775-790, 1991.

BRODATZ, P. Textures: A Photographic Album for Artists \& Designers. New York: Dover Publications, 1966

CHANDRA, D. V. S. Target orientation estimation using Fourier energy spectrum. IEEE Transactions on Aerospace and Electronic Systems, v. 34, n. 3, p. 1009-1012, 1998.

CHEN, Jia-Lin; KUNDU, Amlan. Rotation and gray scale transform invariant texture identification using wavelet decomposition and hidden Markov model. IEEE Transactions on Pattern Analysis and Machine Intelligence, v. 16, n. 2, p. 208-214, 1994.

CHEN, Chen et al. Land-use scene classification using multi-scale completed local binary patterns. Signal, image and video processing, v. 10, n. 4, p. 745-752, 2016. 
CHIERICI, Carlos Eduardo de Oliveira. Classificação de texturas com diferentes orientações baseada em descritores locais. 2015. Dissertação (Mestrado em Processamento de Sinais de Instrumentação) - Escola de Engenharia de São Carlos, Universidade de São Paulo, São Carlos, $2015 . \quad$ doi: 10.11606/D.18.2015.tde-27102015-103555. Acesso em: 2016-07-11.

CLERC, Maurice; KENNEDY, James. The particle swarm-explosion, stability, and convergence in a multidimensional complex space. IEEE transactions on Evolutionary Computation, v. 6, n. 1, p. 58-73, 2002.

DALAL, Navneet; TRIGGS, Bill. Histograms of oriented gradients for human detection. In: Computer Vision and Pattern Recognition, 2005. CVPR 2005. IEEE Computer Society Conference on. IEEE, 2005. p. 886-893.

DAS, Paramita et al. A texture based approach for automatic identification of benign and malignant tumor from FNAC images. In: Recent Trends in Information Systems (ReTIS), 2015 IEEE 2nd International Conference on. IEEE, 2015. p. 249-254.

DONG, Yongsheng et al. Multiscale Sampling Based Texture Image Classification. IEEE Signal Processing Letters, v. 24, n. 5, p. 614-618, 2017.

FERNÁNDEZ, Antonio et al. Evaluation of robustness against rotation of LBP, CCR and ILBP features in granite texture classification. Machine vision and Applications, v. 22, n. 6, p. 913-926, 2011.

FERRAZ, Carolina Toledo; GONZAGA, Adilson. Object classification using a local texture descriptor and a support vector machine. Multimedia Tools and Applications, p. 1-33, 2016.

FERRAZ, Carolina Toledo; PEREIRA JR, Osmando; GONZAGA, Adilson. Feature description based on center-symmetric local mapped patterns. In: Proceedings of the 29th Annual ACM Symposium on Applied Computing. ACM, 2014. p. 39-44. 
GUO, Zhenhua; ZHANG, Lei; ZHANG, David. A completed modeling of local binary pattern operator for texture classification. IEEE Transactions on Image Processing, v. 19, n. 6, p. 1657-1663, 2010a.

A completed modeling of local binary pattern operator for texture classification. IEEE Transactions on Image Processing, v. 19, n. 6, p. 1657-1663, 2010 b.

HARALICK, R.; SHANMUGAM, K.; DINSTEIN, I. Textural features for image classification. IEEE Transactions on systems, man, and cybernetics, v. 3, n. 6, p. 610-621, 1973.

HE, Dong-Chen; WANG, Li. Texture unit, texture spectrum, and texture analysis. IEEE transactions on Geoscience and Remote Sensing, v. 28, n. 4, p. 509-512, 1990.

HE, Xiangjian et al. Local binary patterns for human detection on hexagonal structure. In: Multimedia, 2007. ISM 2007. Ninth IEEE International Symposium on. IEEE, 2007. p. $65-71$.

HEIKKILÄ, Marko; PIETIKÄINEN, Matti; SCHMID, Cordelia. Description of interest regions with center-symmetric local binary patterns. In: Computer vision, graphics and image processing. Springer Berlin Heidelberg, 2006. p. 58-69.

HEIKKILÄ, Marko; PIETIKÄINEN, Matti; SCHMID, Cordelia. Description of interest regions with local binary patterns. Pattern recognition, v. 42, n. 3, p. 425-436, 2009.

HEPPNER, F.; GRENANDER, U. A stochastic nonlinear model for coordinated bird flocks The ubiquity of chaos, AAAS Publications, 1990, 233-238

HOFFMEYER, J. The Swarming body. In: Semiotics around the World; Procedings of the Fifith Congresso f the International Association for Semiotic Studies; Berkley, 1994.

HU, Hsien-Chih et al. Local Binary Pattern Special Investigation based on Search image face texture recognition. In: Computer, Consumer and Control (IS3C), 2016 International Symposium on. IEEE, 2016. p. 686-689. 
HUANG, Xiangsheng; LI, Stan Z.; WANG, Yangsheng. Shape localization based on statistical method using extended local binary pattern. In: Image and Graphics (ICIG'04), Third International Conference on. IEEE, 2004. p. 184-187.

HUANG, Di et al. HSOG: a novel local image descriptor based on histograms of the secondorder gradients. IEEE Transactions on Image Processing, v. 23, n. 11, p. 4680-4695, 2014.

JAFARI-KHOUZANI, Kourosh; SOLTANIAN-ZADEH, Hamid. Radon transform orientation estimation for rotation invariant texture analysis. IEEE Transactions on Pattern Analysis and Machine Intelligence, v. 27, n. 6, p. 1004-1008, 2005.

JIN, Hongliang et al. Face detection using improved LBP under Bayesian framework. In: Image and Graphics (ICIG'04), Third International Conference on. IEEE, 2004. p. 306-309.

KASHYAP, Rangasami L.; KHOTANZAD, Alireza. A model-based method for rotation invariant texture classification. IEEE Transactions on Pattern Analysis and Machine Intelligence, n. 4, p. 472-481, 1986.

KENNEDY, J.; EBERHART, R. Particle swarm optimization. In; Neural Networks ,IEEE International Conference on, 1995, p.1942-1948, v.4 .

KYLBERG, Gustaf. Automatic virus identification using TEM: image segmentation and texture analysis. 2014. Tese de Doutorado. Acta Universitatis Upsaliensis.

KYLBERG, Gustaf; SINTORN, Ida-Maria Kylberg Sintorn Rotation dataset (2015). http:// www.cb.uu.se/ gustaf/KylbergSintornRotation/. Acessado 20 Fev 2017

On the influence of interpolation method on rotation invariance in texture recognition. EURASIP Journal on Image and Video Processing, v. 2016, n. 1, p. 17, 2016. 
LATEGAHN, Henning et al. Texture classification by modeling joint distributions of local patterns with Gaussian mixtures. IEEE Transactions on Image Processing, v. 19, n. 6, p. 1548-1557, 2010.

LATIF, M. H. et al. Texture descriptors based affective states recognition-frontal face thermal image. In: Biomedical Engineering and Sciences (IECBES), 2016 IEEE EMBS Conference on. IEEE, 2016. p. 80-85.

LIAO, Shu; LAW, Max WK; CHUNG, Albert CS. Dominant local binary patterns for texture classification. IEEE transactions on image processing, v. 18, n. 5, p. 1107-1118, 2009.

LIU, Li et al. Local binary features for texture classification: Taxonomy and experimental study. Pattern Recognition, v. 62, p. 135-160, 2017.

LUENGO, Cris. DIPimage, a Matlab toolbox for scientific image processing and analysis. http://www.diplib.org/. Accesso 20 fev 2017

MAANI, Rouzbeh; KALRA, Sanjay; YANG, Yee-Hong. Rotation invariant local frequency descriptors for texture classification. IEEE Transactions on Image Processing, v. 22, n. 6, p. 2409-2419, 2013.

MÄENPÄÄ, T.: The local binary pattern approach to texture analysis-extensions and applications. PhD thesis, Acta Universitatis Ouluensis C 187, University of Oulu (2003)

MANDAL, Devraj; CHATTERJEE, Amitava; MAITRA, Madhubanti. Particle Swarm Optimization Based Fast Chan-Vese Algorithm for Medical Image Segmentation. In: Metaheuristics for Medicine and Biology. Springer Berlin Heidelberg, 2017. p. 49-74.

MARCOS, Diego; VOLPI, Michele; TUIA, Devis. Learning rotation invariant convolutional filters for texture classification. In: Pattern Recognition (ICPR), 2016 23rd International Conference on. IEEE, 2016. p. 2012-2017. 
MESTER, Rudolf. Orientation estimation: Conventional techniques and a new nondifferential approach. In: Signal Processing Conference, 2000 10th European. IEEE, 2000. p. $1-4$.

MILLONAS, M. M. Swarms, phase transitions, and collective intelligence Artificial Life III, Addison-Wesley, 1994

MORALES, Sandra et al. Retinal disease screening through local binary patterns. IEEE journal of biomedical and health informatics, v. 21, n. 1, p. 184-192, 2017.

NANNI, Loris; BRAHNAM, Sheryl; LUMINI, Alessandra. A local approach based on a Local Binary Patterns variant texture descriptor for classifying pain states. Expert Systems with Applications, v. 37, n. 12, p. 7888-7894, 2010.

NEGRI, T. et al. A Robust Descriptor for Color Texture Classification Under Varying Illumination. In Computer Vision, Imaging and Computer Graphics Theory and Applications (VISIGRAPP), 2017 12th International Joint Conference on. 2017. v. 4, p. 378-388.

NGUYEN, Vu-Lam et al. An integrated descriptor for texture classification. In: Pattern Recognition (ICPR), 2016 23rd International Conference on. IEEE, 2016. p. 2006-2011.

OJALA, Timo et al. Outex-new framework for empirical evaluation of texture analysis algorithms. In: Pattern Recognition, 2002. Proceedings. 16th International Conference on. IEEE, 2002. p. 701-706.

OJALA, Timo; PIETIKÄINEN, Matti; HARWOOD, David. A comparative study of texture measures with classification based on featured distributions. Pattern recognition, v. 29, n. 1, p. 51-59, 1996.

OJALA, Timo; PIETIKAINEN, Matti; MAENPAA, Topi. Multiresolution gray-scale and rotation invariant texture classification with local binary patterns. IEEE Transactions on pattern analysis and machine intelligence, v. 24, n. 7, p. 971-987, 2002. 
PAN, Zhibin et al. Local Adaptive Binary Patterns Using Diamond Sampling Structure for Texture Classification. IEEE Signal Processing Letters, v. 24, n. 6, p. 828-832, 2017.

PIETIKÄINEN, Matti et al. Local binary patterns for still images. Computer Vision Using Local Binary Patterns Springer London, 2011, 40, 13-47.

PIETIKÄINEN, M.; ZHAO, G.; HADID, A.; AHONEN, T. Computer Vision Using Local Binary Patterns Springer, p.209, 2011.

POLI, Riccardo; KENNEDY, James; BLACKWELL, Tim. Particle swarm optimization. Swarm intelligence, v. 1, n. 1, p. 33-57, 2007.

PRIBADI, Kukuh Daud et al. Dynamic response of maximum power point tracking using particle swarm optimization for wind energy conversion system. In: Information Technology and Electrical Engineering (ICITEE), 2016 8th International Conference on. IEEE, 2016. p. 1-6.

RASSEM, Taha H. et al. Multi-Scale Colour Completed Local Binary Patterns for Scene and Event Sport Image Categorisation. IAENG International Journal of Computer Science,, v. 44, n. 2, p. 197-211, 2017.

REN, Jianfeng; JIANG, Xudong; YUAN, Junsong. LBP-Structure Optimization With Symmetry and Uniformity Regularizations for Scene Classification. IEEE Signal Processing Letters, v. 24, n. 1, p. 37-41, 2017.

REYNOLDS, Craig W. Flocks, herds and schools: A distributed behavioral model. ACM SIGGRAPH computer graphics, v. 21, n. 4, p. 25-34, 1987.

RYU, Jongbin; HONG, Sungeun; YANG, Hyun S. Sorted consecutive local binary pattern for texture classification. IEEE Transactions on Image Processing, v. 24, n. 7, p. 2254-2265, 2015. 
SERAPIÃO, Adriane Beatriz de Souza. Fundamentos de otimização por inteligência de enxames: uma visão geral. Sba: Controle \& Automação Sociedade Brasileira de Automatica, v. 20, n. 3, p. 271-304, 2009.

SHAIKH, Nuzhat Faiz; DOYE, D. D. Improving the accuracy of iris recognition system using neural network and particle swarm optimization. International Journal of Computer Applications, v. 79, n. 3, 2013.

SHANG, Jun et al. Object recognition using rotation invariant local binary pattern of significant bit planes. IET Image Processing, v. 10, n. 9, p. 662-670, 2016.

SHI, Yuhui. Particle swarm optimization. IEEE Connections, v. 2, n. 1, p. 8-13, 2004.

SHI, Yuhui; EBERHART, Russell. Parameter selection in particle swarm optimization. In: Evolutionary programming VII. Springer Berlin/Heidelberg, 1998a. p. 591-600.

A modified particle swarm optimizer. In: Evolutionary Computation Proceedings, 1998. IEEE World Congress on Computational Intelligence., The 1998 IEEE International Conference on. IEEE, 1998. p. 69-73.

SONG, Tiecheng et al. L2SSP: Robust keypoint description using local second-order statistics with soft-pooling. Neurocomputing, v. 230, p. 230-242, 2017a.

SONG, Tiecheng et al. LETRIST: Locally Encoded Transform Feature Histogram for Rotation-Invariant Texture Classification. IEEE Transactions on Circuits and Systems for Video Technology, $2017 \mathrm{~b}$.

SOUZA, Jones Mendonça; VIEIRA, Raissa Tavares; GONZAGA, Adilson. Analysis of iris texture under pupil contraction/dilation for biometric recognition. In: Workshop de Visão Computacional, 2015, p. 128-133.

TAN, Xiaoyang; TRIGGS, Bill. Enhanced local texture feature sets for face recognition under difficult lighting conditions. In: International Workshop on Analysis and Modeling of Faces and Gestures. Springer Berlin Heidelberg, 2007. p. 168-182. 
TOPI, Mäenpää et al. Robust texture classification by subsets of local binary patterns. In: Pattern Recognition, 2000. Proceedings. 15th International Conference on. IEEE, 2000. p. 935-938.

TZE KIN TEO, Kenneth et al. Particle Swarm Optimization based Maximum Power Point Tracking for Partially Shaded Photovoltaic Arrays. International Journal of Simulation-Systems, Science \& Technology, v. 17, n. 34, 2016.

VARMA, Manik; GARG, Rahul. Locally invariant fractal features for statistical texture classification. In: Computer Vision, 2007. ICCV 2007. IEEE 11th International Conference on. IEEE, 2007. p. 1-8.

VARMA, Manik; ZISSERMAN, Andrew. A statistical approach to texture classification from single images. International journal of computer vision, v. 62, n. 1, p. 61-81, 2005

VIEIRA, Raissa Tavares. Análise de micropadrões em imagens digitais baseada em números fuzzy. 2013. Dissertação (Mestrado em Processamento de Sinais de Instrumentação) - Escola de Engenharia de São Carlos, Universidade de São Paulo, São Carlos, 2013. doi:10.11606/D.18.2013.tde-29042013-154729. Acesso em: 2017-06-12.

VIEIRA, Raissa et al. Local fuzzy pattern: a new way for micro-pattern analysis. Intelligent Data Engineering and Automated Learning-IDEAL 2012, p. 602-611, 2012.

VIEIRA, Raissa Tavares; NEGRI, Tamiris Trevisan; GONZAGA, Adilson. Robustness of Rotation Invariant Descriptors for Texture Classification. In: International Symposium on Visual Computing. Springer International Publishing, 2016. p. 268-277.

XU, Gang; GAO, Jie. Weak signal detection based on a new matching pursuit method. In: Machine Learning and Cybernetics, 2008 International Conference on. IEEE, 2008. p. 1036-1040.

ZHAO, Guoying et al. Rotation-invariant image and video description with local binary pattern features. IEEE Transactions on Image Processing, v. 21, n. 4, p. 1465-1477, 2012. 
ZEPPELZAUER, Matthias et al. Topological descriptors for 3D surface analysis. In: International Workshop on Computational Topology in Image Context. Springer International Publishing, 2016. p. 77-87. 


\section{APÊNDICE A - Acurácias médias obtidas pelos descritores avaliados no Experimento 1}

\begin{tabular}{|c|c|c|c|c|c|c|c|c|c|c|c|c|}
\hline & Dim. & & Média & $\begin{array}{l}\text { Desvio } \\
\text { padrão }\end{array}$ & $40^{\circ}$ & $80^{\circ}$ & $120^{\circ}$ & $160^{\circ}$ & $200^{\circ}$ & $240^{\circ}$ & $280^{\circ}$ & $320^{\circ}$ \\
\hline \multirow{6}{*}{ 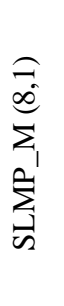 } & \multirow{6}{*}{$\bar{\nabla}$} & HW & $91,05 \%$ & 0,019 & $89,28 \%$ & $89,05 \%$ & $89,76 \%$ & $93,54 \%$ & $93,60 \%$ & $92,02 \%$ & $88,53 \%$ & $92,64 \%$ \\
\hline & & NN & $47,62 \%$ & 0,201 & $28,73 \%$ & $79,49 \%$ & $32,65 \%$ & $48,68 \%$ & $48,82 \%$ & $33,48 \%$ & $80,28 \%$ & $28,79 \%$ \\
\hline & & $\mathrm{LN}$ & $34,45 \%$ & 0,027 & $31,67 \%$ & $34,60 \%$ & $38,86 \%$ & $32,76 \%$ & $32,69 \%$ & $38,81 \%$ & $34,55 \%$ & $31,68 \%$ \\
\hline & & $\mathrm{CB}$ & $75,20 \%$ & 0,009 & $73,95 \%$ & $75,98 \%$ & $75,96 \%$ & $74,89 \%$ & $74,80 \%$ & $75,65 \%$ & $76,39 \%$ & $73,96 \%$ \\
\hline & & SP & $91,99 \%$ & 0,009 & $90,28 \%$ & $92,76 \%$ & $92,21 \%$ & $92,60 \%$ & $91,98 \%$ & $91,85 \%$ & $93,35 \%$ & $90,88 \%$ \\
\hline & & $\mathrm{LZ}$ & $94,73 \%$ & 0,008 & $93,53 \%$ & $95,36 \%$ & $94,64 \%$ & $95,33 \%$ & $95,09 \%$ & $94,61 \%$ & $95,84 \%$ & $93,45 \%$ \\
\hline \multirow{6}{*}{ 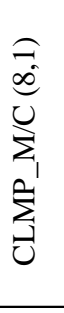 } & \multirow{6}{*}{ ๙ి } & HW & $87,23 \%$ & 0,017 & $86,11 \%$ & $84,78 \%$ & $87,71 \%$ & $88,32 \%$ & $88,29 \%$ & $88,54 \%$ & $84,24 \%$ & $89,81 \%$ \\
\hline & & $\mathrm{NN}$ & $77,12 \%$ & 0,085 & $68,16 \%$ & $89,36 \%$ & $70,19 \%$ & $80,88 \%$ & $80,84 \%$ & $70,31 \%$ & $89,07 \%$ & $68,15 \%$ \\
\hline & & $\mathrm{LN}$ & $75,30 \%$ & 0,009 & $73,71 \%$ & $75,91 \%$ & $76,01 \%$ & $75,30 \%$ & $75,16 \%$ & $76,29 \%$ & $76,08 \%$ & $73,96 \%$ \\
\hline & & $\mathrm{CB}$ & $86,88 \%$ & 0,006 & $86,51 \%$ & $88,00 \%$ & $86,88 \%$ & $86,67 \%$ & $86,86 \%$ & $86,90 \%$ & $87,43 \%$ & $85,77 \%$ \\
\hline & & SP & $90,76 \%$ & 0,004 & $90,68 \%$ & $91,20 \%$ & $90,57 \%$ & $90,62 \%$ & $90,56 \%$ & $90,92 \%$ & $91,35 \%$ & $90,17 \%$ \\
\hline & & LZ & $90,53 \%$ & 0,024 & $91,32 \%$ & $91,91 \%$ & $91,14 \%$ & $84,26 \%$ & $91,41 \%$ & $91,47 \%$ & $91,76 \%$ & $91,00 \%$ \\
\hline \multirow{6}{*}{ 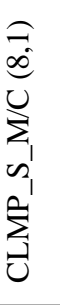 } & \multirow{6}{*}{ के } & HW & $94,11 \%$ & 0,012 & $92,39 \%$ & $92,01 \%$ & $94,38 \%$ & $95,03 \%$ & $95,09 \%$ & $93,88 \%$ & $94,08 \%$ & $96,04 \%$ \\
\hline & & NN & $84,89 \%$ & 0,081 & $75,67 \%$ & $95,92 \%$ & $78,82 \%$ & $89,28 \%$ & $89,29 \%$ & $78,71 \%$ & $95,65 \%$ & $75,74 \%$ \\
\hline & & LN & $70,19 \%$ & 0,016 & $68,82 \%$ & $70,95 \%$ & $72,08 \%$ & $68,61 \%$ & $68,72 \%$ & $72,72 \%$ & $70,99 \%$ & $68,59 \%$ \\
\hline & & CB & $88,54 \%$ & 0,009 & $87,75 \%$ & $90,31 \%$ & $87,58 \%$ & $88,40 \%$ & $88,10 \%$ & $88,72 \%$ & $89,65 \%$ & $87,80 \%$ \\
\hline & & SP & $94,92 \%$ & 0,005 & $94,38 \%$ & $95,74 \%$ & $94,66 \%$ & $95,04 \%$ & $94,72 \%$ & $94,83 \%$ & $95,50 \%$ & $94,46 \%$ \\
\hline & & LZ & $95,73 \%$ & 0,004 & $95,38 \%$ & $96,46 \%$ & $95,32 \%$ & $96,00 \%$ & $95,59 \%$ & $95,53 \%$ & $96,21 \%$ & $95,32 \%$ \\
\hline \multirow{6}{*}{ 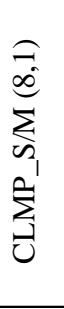 } & \multirow{6}{*}{ 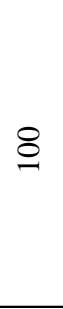 } & HW & $91,95 \%$ & 0,019 & $90,23 \%$ & $89,13 \%$ & $93,27 \%$ & $93,71 \%$ & $93,69 \%$ & $93,49 \%$ & $89,02 \%$ & $93,07 \%$ \\
\hline & & $\mathrm{NN}$ & $42,57 \%$ & 0,194 & $25,12 \%$ & $73,98 \%$ & $27,74 \%$ & $43,05 \%$ & $43,28 \%$ & $27,90 \%$ & $73,99 \%$ & $25,47 \%$ \\
\hline & & $\mathrm{LN}$ & $33,50 \%$ & 0,02 & $32,61 \%$ & $32,53 \%$ & $36,73 \%$ & $33,51 \%$ & $31,78 \%$ & $36,80 \%$ & $31,07 \%$ & $32,97 \%$ \\
\hline & & $\mathrm{CB}$ & $68,03 \%$ & 0,007 & $67,03 \%$ & $68,65 \%$ & $68,72 \%$ & $67,92 \%$ & $67,22 \%$ & $68,38 \%$ & $68,83 \%$ & $67,50 \%$ \\
\hline & & SP & $87,18 \%$ & 0,007 & $86,13 \%$ & $88,02 \%$ & $87,23 \%$ & $87,48 \%$ & $87,57 \%$ & $86,90 \%$ & $88,04 \%$ & $86,09 \%$ \\
\hline & & $\mathrm{LZ}$ & $90,64 \%$ & 0,007 & $89,76 \%$ & $91,40 \%$ & $90,21 \%$ & $90,90 \%$ & $91,03 \%$ & $90,46 \%$ & $91,69 \%$ & $89,68 \%$ \\
\hline \multirow{6}{*}{ 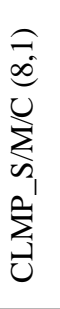 } & \multirow{6}{*}{ ఠి } & HW & $92,39 \%$ & 0,017 & $90,60 \%$ & $88,69 \%$ & $92,88 \%$ & $93,24 \%$ & $94,07 \%$ & $93,62 \%$ & $91,79 \%$ & $94,24 \%$ \\
\hline & & NN & $57,29 \%$ & 0,18 & $40,70 \%$ & $86,31 \%$ & $43,89 \%$ & $58,17 \%$ & $57,71 \%$ & $44,30 \%$ & $86,59 \%$ & $40,61 \%$ \\
\hline & & $\mathrm{LN}$ & $42,66 \%$ & 0,017 & $41,09 \%$ & $42,68 \%$ & $45,59 \%$ & $41,40 \%$ & $41,56 \%$ & $45,24 \%$ & $42,84 \%$ & $40,91 \%$ \\
\hline & & $\mathrm{CB}$ & $76,03 \%$ & 0,009 & $74,54 \%$ & $77,40 \%$ & $76,65 \%$ & $76,26 \%$ & $75,64 \%$ & $76,07 \%$ & $76,82 \%$ & $74,87 \%$ \\
\hline & & SP & $91,85 \%$ & 0,007 & $90,87 \%$ & $92,60 \%$ & $91,64 \%$ & $92,16 \%$ & $92,09 \%$ & $92,10 \%$ & $92,60 \%$ & $90,75 \%$ \\
\hline & & LZ & $94,31 \%$ & 0,005 & $93,69 \%$ & $95,00 \%$ & $94,12 \%$ & $94,54 \%$ & $94,41 \%$ & $94,34 \%$ & $94,79 \%$ & $93,61 \%$ \\
\hline \multirow{6}{*}{ 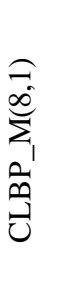 } & \multirow{6}{*}{ 인 } & HW & $66,62 \%$ & 0,037 & $66,64 \%$ & $68,74 \%$ & $64,53 \%$ & $70,42 \%$ & $72,58 \%$ & $58,54 \%$ & $66,16 \%$ & $65,37 \%$ \\
\hline & & NN & $43,47 \%$ & 0,126 & $35,01 \%$ & $64,14 \%$ & $35,49 \%$ & $40,79 \%$ & $40,26 \%$ & $34,60 \%$ & $65,31 \%$ & $32,15 \%$ \\
\hline & & $\mathrm{LN}$ & $30,74 \%$ & 0,029 & $26,52 \%$ & $34,49 \%$ & $30,60 \%$ & $31,44 \%$ & $30,78 \%$ & $31,83 \%$ & $34,20 \%$ & $26,06 \%$ \\
\hline & & CB & $45,84 \%$ & 0,045 & $42,21 \%$ & $52,45 \%$ & $42,90 \%$ & $46,77 \%$ & $47,87 \%$ & $40,96 \%$ & $52,60 \%$ & $40,96 \%$ \\
\hline & & SP & $58,40 \%$ & 0,049 & $52,64 \%$ & $65,05 \%$ & $55,54 \%$ & $59,42 \%$ & $60,47 \%$ & $55,66 \%$ & $66,12 \%$ & $52,26 \%$ \\
\hline & & LZ & $64,25 \%$ & 0,046 & $57,42 \%$ & $70,01 \%$ & $59,45 \%$ & $65,12 \%$ & $66,24 \%$ & $61,08 \%$ & $70,19 \%$ & $57,69 \%$ \\
\hline
\end{tabular}




\begin{tabular}{|c|c|c|c|c|c|c|c|c|c|c|c|c|}
\hline & Dim. & & Média & $\begin{array}{l}\text { Desvio } \\
\text { padrão }\end{array}$ & $40^{\circ}$ & $80^{\circ}$ & $120^{\circ}$ & $160^{\circ}$ & $200^{\circ}$ & $240^{\circ}$ & $280^{\circ}$ & $320^{\circ}$ \\
\hline \multirow{6}{*}{ 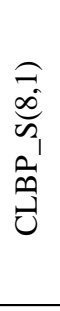 } & & HW & & " 0017 & $74,00 \%$ & $76,28 \%$ & $73,56 \%$ & & $77,36 \%$ & $72,14 \%$ & $73,78 \%$ & $76,60 \%$ \\
\hline & & $\mathrm{NN}$ & & & & & & & & & & \\
\hline & & $\mathrm{LN}$ & & 0,016 & $28,25 \%$ & $32,60 \%$ & $30,46 \%$ & $28,48 \%$ & $28,67 \%$ & & & $28,42 \%$ \\
\hline & & $\mathrm{CB}$ & $58,02 \%$ & 0,034 & $58,96 \%$ & $61,46 \%$ & $57,45 \%$ & $58,12 \%$ & $56,95 \%$ & $50,09 \%$ & $61,79 \%$ & $59,37 \%$ \\
\hline & & SP & & 0,019 & $73,32 \%$ & & $73,63 \%$ & & & & & \\
\hline & & $\mathrm{LZ}$ & $78,75 \%$ & 0,024 & $75,95 \%$ & $82,38 \%$ & $76,48 \%$ & $78,97 \%$ & $78,24 \%$ & & & $76,05 \%$ \\
\hline \multirow{6}{*}{ 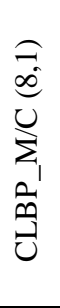 } & \multirow{6}{*}{ ㄱ. } & & & & & & & & & & & \\
\hline & & $\mathrm{NN}$ & $71,08 \%$ & 0,095 & $61,32 \%$ & $84,99 \%$ & $63,20 \%$ & $75,03 \%$ & $74,20 \%$ & $63,59 \%$ & & $61,32 \%$ \\
\hline & & $\mathrm{LN}$ & & 0,015 & $58,00 \%$ & $58,59 \%$ & $60,91 \%$ & & $57,48 \%$ & & & $57,60 \%$ \\
\hline & & $\mathrm{CB}$ & $73,41 \%$ & 0,01 & $73,64 \%$ & $75,04 \%$ & $72,60 \%$ & $72,82 \%$ & $72,76 \%$ & $72,60 \%$ & $74,97 \%$ & $72,84 \%$ \\
\hline & & SP & & 0,015 & $79,09 \%$ & & $78,52 \%$ & & & & & \\
\hline & & $\mathrm{LZ}$ & & 0,015 & & & $80,68 \%$ & & & & & \\
\hline \multirow{6}{*}{ 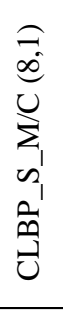 } & \multirow{6}{*}{ ஜి } & & & & & & & & & & & \\
\hline & & $\mathrm{NN}$ & & 0,138 & $30,55 \%$ & & $34,59 \%$ & & & & & $71 \%$ \\
\hline & & $\mathrm{LN}$ & $32,57 \%$ & 0,016 & $31,24 \%$ & $33,54 \%$ & $34,82 \%$ & $30,87 \%$ & $31,01 \%$ & $34,91 \%$ & & $31,23 \%$ \\
\hline & & $\mathrm{CB}$ & & & & & $61,38 \%$ & & & & & $62,71 \%$ \\
\hline & & SP & $81,57 \%$ & 0,015 & $81,68 \%$ & $83,97 \%$ & $80,29 \%$ & $80,36 \%$ & $80,13 \%$ & $80,65 \%$ & $83,90 \%$ & $81,55 \%$ \\
\hline & & $\mathrm{LZ}$ & & & & & & & & & & \\
\hline \multirow{6}{*}{ 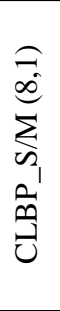 } & \multirow{6}{*}{8} & & & & & & & & & & & \\
\hline & & $\mathrm{NN}$ & $24,62 \%$ & 0,174 & $10,66 \%$ & $53,76 \%$ & $12,54 \%$ & $21,45 \%$ & $22,09 \%$ & $12,02 \%$ & $53,84 \%$ & $10,56 \%$ \\
\hline & & $\mathrm{LN}$ & & & & & & & & & & \\
\hline & & $\mathrm{CB}$ & $56,49 \%$ & 0,007 & $56,14 \%$ & $57,29 \%$ & $56,26 \%$ & $56,63 \%$ & & & $57,78 \%$ & $55,86 \%$ \\
\hline & & SP & & & & & & & & & & $77,60 \%$ \\
\hline & & $\mathrm{LZ}$ & & & & & & & & & & $30 \%$ \\
\hline \multirow{6}{*}{ 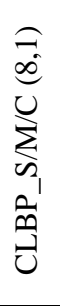 } & \multirow{6}{*}{ ஓ্ণ } & & & & & & & & & & & \\
\hline & & $\mathrm{NN}$ & & & & & & & & & & \\
\hline & & $\mathrm{LN}$ & & 0,048 & & $40,82 \%$ & & $40,81 \%$ & $40,48 \%$ & $54,36 \%$ & $40,37 \%$ & \\
\hline & & $\mathrm{CB}$ & $78,42 \%$ & 0,006 & $77,92 \%$ & $79,27 \%$ & $78,62 \%$ & $78,74 \%$ & $77,96 \%$ & $78,69 \%$ & $78,76 \%$ & $77,37 \%$ \\
\hline & & SP & & & & & & $92,64 \%$ & & & ,04\% & $91,19 \%$ \\
\hline & & $\mathrm{LZ}$ & & & & & & & & & & \\
\hline \multirow{6}{*}{ 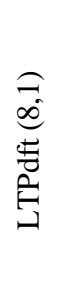 } & \multirow{6}{*}{ ñ } & & & 0,034 & $84,10 \%$ & $91,10 \%$ & $86,30 \%$ & $96,10 \%$ & $93,50 \%$ & $89,30 \%$ & $87,50 \%$ & $88,50 \%$ \\
\hline & & $\mathrm{NN}$ & & 0,232 & $20,30 \%$ & $79,20 \%$ & $22,10 \%$ & $37,20 \%$ & $38,00 \%$ & $22,40 \%$ & $76,80 \%$ & $20,40 \%$ \\
\hline & & $\mathrm{LN}$ & $46,13 \%$ & 0,036 & $41,00 \%$ & $48,70 \%$ & $49,80 \%$ & $46,30 \%$ & $45,50 \%$ & $49,90 \%$ & $47,90 \%$ & $39,90 \%$ \\
\hline & & $\mathrm{CB}$ & & 0,025 & & & & & & & & $78,70 \%$ \\
\hline & & SP & & & $90,70 \%$ & $96,80 \%$ & $92,60 \%$ & $95,80 \%$ & $94,30 \%$ & $94,40 \%$ & $96,80 \%$ & $92,60 \%$ \\
\hline & & $\mathrm{LZ}$ & $95,56 \%$ & 0,018 & $92,80 \%$ & $97,90 \%$ & $93,60 \%$ & $97,10 \%$ & $95,50 \%$ & $95,60 \%$ & $97,80 \%$ & $94,20 \%$ \\
\hline
\end{tabular}




\begin{tabular}{|c|c|c|c|c|c|c|c|c|c|c|c|c|}
\hline & Dim. & & Média & $\begin{array}{l}\text { Desvio } \\
\text { padrão }\end{array}$ & $40^{\circ}$ & $80^{\circ}$ & $120^{\circ}$ & $160^{\circ}$ & $200^{\circ}$ & $240^{\circ}$ & $280^{\circ}$ & $320^{\circ}$ \\
\hline \multirow{6}{*}{ 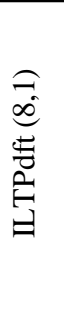 } & \multirow{6}{*}{$\overline{8}$} & HW & $90,44 \%$ & 0,032 & $87,90 \%$ & $94,80 \%$ & $86,40 \%$ & $94,10 \%$ & $90,50 \%$ & $85,10 \%$ & $91,10 \%$ & $93,60 \%$ \\
\hline & & NN & $61,46 \%$ & 0,198 & $43,90 \%$ & $95,60 \%$ & $47,60 \%$ & $60,40 \%$ & $60,60 \%$ & $46,50 \%$ & $92,60 \%$ & $44,50 \%$ \\
\hline & & LN & $64,14 \%$ & 0,024 & $64,30 \%$ & $66,60 \%$ & $65,50 \%$ & $62,50 \%$ & $59,70 \%$ & $62,20 \%$ & $67,30 \%$ & $65,00 \%$ \\
\hline & & $\mathrm{CB}$ & $83,46 \%$ & 0,033 & $84,40 \%$ & $87,50 \%$ & $81,10 \%$ & $84,10 \%$ & $80,10 \%$ & $77,80 \%$ & $88,10 \%$ & $84,60 \%$ \\
\hline & & SP & $91,70 \%$ & 0,039 & $92,10 \%$ & $96,40 \%$ & $87,60 \%$ & $92,80 \%$ & $88,80 \%$ & $85,30 \%$ & $96,90 \%$ & $93,70 \%$ \\
\hline & & $\mathrm{LZ}$ & $92,39 \%$ & 0,041 & $92,60 \%$ & $97,70 \%$ & $87,80 \%$ & $93,50 \%$ & $89,90 \%$ & $85,70 \%$ & $97,80 \%$ & $94,10 \%$ \\
\hline \multirow{6}{*}{ 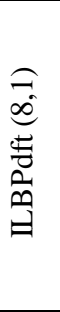 } & \multirow{6}{*}{$\stackrel{2}{2}$} & HW & $79,46 \%$ & 0,054 & $71,80 \%$ & $88,60 \%$ & $74,30 \%$ & $87,30 \%$ & $78,40 \%$ & $76,40 \%$ & $83,00 \%$ & $75,90 \%$ \\
\hline & & $\mathrm{NN}$ & $33,14 \%$ & 0,226 & $17,10 \%$ & $73,70 \%$ & $17,70 \%$ & $25,80 \%$ & $25,90 \%$ & $17,50 \%$ & $70,00 \%$ & $17,40 \%$ \\
\hline & & LN & $28,55 \%$ & 0,072 & $23,80 \%$ & $40,40 \%$ & $24,50 \%$ & $29,20 \%$ & $22,70 \%$ & $25,40 \%$ & $40,60 \%$ & $21,80 \%$ \\
\hline & & CB & $64,38 \%$ & 0,069 & $60,40 \%$ & $76,30 \%$ & $59,30 \%$ & $66,10 \%$ & $59,10 \%$ & $59,20 \%$ & $75,10 \%$ & $59,50 \%$ \\
\hline & & SP & $79,79 \%$ & 0,071 & $74,90 \%$ & $91,60 \%$ & $74,40 \%$ & $82,50 \%$ & $75,70 \%$ & $73,70 \%$ & $91,00 \%$ & $74,50 \%$ \\
\hline & & LZ & $82,24 \%$ & 0,072 & $77,60 \%$ & $93,70 \%$ & $76,50 \%$ & $84,60 \%$ & $78,50 \%$ & $76,40 \%$ & $94,30 \%$ & $76,30 \%$ \\
\hline \multirow{6}{*}{\multicolumn{2}{|c|}{ 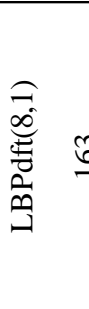 }} & $\mathrm{HW}$ & $79,40 \%$ & 0,04 & $72,80 \%$ & $82,50 \%$ & $75,90 \%$ & $85,20 \%$ & $83,10 \%$ & $82,90 \%$ & $75,20 \%$ & $77,60 \%$ \\
\hline & & $\mathrm{NN}$ & $31,00 \%$ & 0,188 & $15,70 \%$ & $64,80 \%$ & $18,20 \%$ & $27,20 \%$ & $27,20 \%$ & $17,80 \%$ & $60,60 \%$ & $16,50 \%$ \\
\hline & & LN & $22,26 \%$ & 0,032 & $18,20 \%$ & $27,50 \%$ & $21,80 \%$ & $22,70 \%$ & $19,00 \%$ & $23,50 \%$ & $26,20 \%$ & $19,20 \%$ \\
\hline & & CB & $58,15 \%$ & 0,064 & $49,00 \%$ & $68,20 \%$ & $52,00 \%$ & $59,30 \%$ & $57,20 \%$ & $58,70 \%$ & $67,20 \%$ & $53,60 \%$ \\
\hline & & SP & $81,38 \%$ & 0,059 & $72,60 \%$ & $89,20 \%$ & $75,80 \%$ & $85,20 \%$ & $81,80 \%$ & $82,50 \%$ & $88,70 \%$ & $75,20 \%$ \\
\hline & & LZ & $85,18 \%$ & 0,056 & $76,90 \%$ & $92,20 \%$ & $79,80 \%$ & $88,40 \%$ & $86,00 \%$ & $86,70 \%$ & $92,40 \%$ & $79,00 \%$ \\
\hline
\end{tabular}




\section{APÊNDICE B - Acurácias médias obtidas pelos descritores avaliados no Experimento 2}

\begin{tabular}{|c|c|c|c|c|c|c|c|c|c|c|c|c|}
\hline & Dim. & & Média & $\begin{array}{l}\text { Desvio } \\
\text { padrão }\end{array}$ & $40^{\circ}$ & $80^{\circ}$ & $120^{\circ}$ & $160^{\circ}$ & $200^{\circ}$ & $240^{\circ}$ & $280^{\circ}$ & $320^{\circ}$ \\
\hline \multirow{6}{*}{ 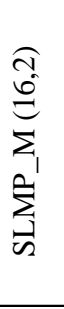 } & \multirow{6}{*}{ สิ } & HW & $90,26 \%$ & 0,021 & $86,81 \%$ & $88,57 \%$ & $89,74 \%$ & $92,83 \%$ & $94,22 \%$ & $90,92 \%$ & $88,86 \%$ & $90,09 \%$ \\
\hline & & NN & $75,61 \%$ & 0,034 & $71,97 \%$ & $81,24 \%$ & $77,21 \%$ & $72,62 \%$ & $72,81 \%$ & $75,70 \%$ & $80,92 \%$ & $72,44 \%$ \\
\hline & & $\mathrm{LN}$ & $65,30 \%$ & 0,017 & $63,04 \%$ & $64,07 \%$ & $68,06 \%$ & $64,74 \%$ & $65,76 \%$ & $68,13 \%$ & $65,17 \%$ & $63,40 \%$ \\
\hline & & $\mathrm{CB}$ & $92,54 \%$ & 0,005 & $91,57 \%$ & $93,18 \%$ & $92,33 \%$ & $92,80 \%$ & $92,78 \%$ & $92,64 \%$ & $93,19 \%$ & $91,85 \%$ \\
\hline & & SP & $95,79 \%$ & 0,005 & $94,98 \%$ & $96,35 \%$ & $95,59 \%$ & $96,21 \%$ & $96,27 \%$ & $95,54 \%$ & $96,30 \%$ & $95,07 \%$ \\
\hline & & $\mathrm{LZ}$ & $96,02 \%$ & 0,007 & $94,91 \%$ & $96,68 \%$ & $95,84 \%$ & $96,51 \%$ & $96,78 \%$ & $95,76 \%$ & $96,64 \%$ & $95,04 \%$ \\
\hline \multirow{6}{*}{ 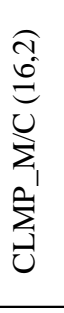 } & \multirow{6}{*}{ ల } & HW & $88,20 \%$ & 0,015 & $86,12 \%$ & $85,28 \%$ & $89,39 \%$ & $89,53 \%$ & $89,31 \%$ & $89,20 \%$ & $87,43 \%$ & $89,36 \%$ \\
\hline & & $\mathrm{NN}$ & $89,81 \%$ & 0,004 & $89,00 \%$ & $90,63 \%$ & $89,92 \%$ & $90,16 \%$ & $89,69 \%$ & $89,73 \%$ & $89,80 \%$ & $89,53 \%$ \\
\hline & & $\mathrm{LN}$ & $83,24 \%$ & 0,010 & $82,08 \%$ & $83,71 \%$ & $84,49 \%$ & $82,43 \%$ & $82,66 \%$ & $85,01 \%$ & $83,59 \%$ & $81,92 \%$ \\
\hline & & $\mathrm{CB}$ & $89,97 \%$ & 0,002 & $90,01 \%$ & $90,24 \%$ & $90,25 \%$ & $89,84 \%$ & $90,26 \%$ & $89,76 \%$ & $89,90 \%$ & $89,52 \%$ \\
\hline & & SP & $90,47 \%$ & 0,002 & $90,62 \%$ & $90,79 \%$ & $90,71 \%$ & $90,45 \%$ & $90,49 \%$ & $90,37 \%$ & $90,45 \%$ & $89,90 \%$ \\
\hline & & LZ & $89,80 \%$ & 0,021 & $90,85 \%$ & $90,89 \%$ & $90,68 \%$ & $83,85 \%$ & $90,83 \%$ & $90,51 \%$ & $90,33 \%$ & $90,42 \%$ \\
\hline \multirow{6}{*}{ 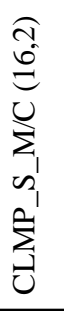 } & \multirow{6}{*}{ in } & HW & $94,32 \%$ & 0,016 & $91,52 \%$ & $91,26 \%$ & $95,27 \%$ & $95,69 \%$ & $95,53 \%$ & $95,20 \%$ & $94,55 \%$ & $95,52 \%$ \\
\hline & & $\mathrm{NN}$ & $87,47 \%$ & 0,016 & $85,88 \%$ & $90,19 \%$ & $88,37 \%$ & $86,32 \%$ & $85,64 \%$ & $87,33 \%$ & $89,82 \%$ & $86,17 \%$ \\
\hline & & LN & $86,91 \%$ & 0,014 & $85,04 \%$ & $87,76 \%$ & $88,61 \%$ & $86,23 \%$ & $86,12 \%$ & $89,10 \%$ & $87,35 \%$ & $85,08 \%$ \\
\hline & & CB & $97,09 \%$ & 0,002 & $97,04 \%$ & $97,38 \%$ & $96,79 \%$ & $97,15 \%$ & $97,06 \%$ & $97,27 \%$ & $96,93 \%$ & $97,09 \%$ \\
\hline & & SP & $97,61 \%$ & 0,001 & $97,59 \%$ & $97,72 \%$ & $97,52 \%$ & $97,62 \%$ & $97,69 \%$ & $97,67 \%$ & $97,46 \%$ & $97,64 \%$ \\
\hline & & LZ & $97,48 \%$ & 0,001 & $97,46 \%$ & $97,52 \%$ & $97,45 \%$ & $97,49 \%$ & $97,55 \%$ & $97,64 \%$ & $97,27 \%$ & $97,48 \%$ \\
\hline \multirow{6}{*}{ 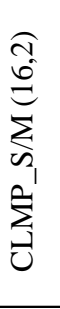 } & \multirow{6}{*}{ त } & HW & $92,69 \%$ & 0,021 & $89,99 \%$ & $89,96 \%$ & $93,65 \%$ & $95,06 \%$ & $95,44 \%$ & $93,65 \%$ & $89,77 \%$ & $94,01 \%$ \\
\hline & & $\mathrm{NN}$ & $87,16 \%$ & 0,028 & $84,30 \%$ & $91,51 \%$ & $89,03 \%$ & $84,74 \%$ & $84,38 \%$ & $86,80 \%$ & $91,68 \%$ & $84,81 \%$ \\
\hline & & $\mathrm{LN}$ & $75,20 \%$ & 0,023 & $72,52 \%$ & $76,12 \%$ & $78,43 \%$ & $72,80 \%$ & $74,56 \%$ & $78,68 \%$ & $76,12 \%$ & $72,36 \%$ \\
\hline & & $\mathrm{CB}$ & $95,07 \%$ & 0,003 & $94,76 \%$ & $95,35 \%$ & $95,35 \%$ & $95,10 \%$ & $95,17 \%$ & $94,97 \%$ & $95,28 \%$ & $94,56 \%$ \\
\hline & & SP & $96,75 \%$ & 0,002 & $96,43 \%$ & $96,80 \%$ & $96,96 \%$ & $96,76 \%$ & $96,98 \%$ & $96,74 \%$ & $96,93 \%$ & $96,43 \%$ \\
\hline & & $\mathrm{LZ}$ & $96,94 \%$ & 0,002 & $96,74 \%$ & $97,02 \%$ & $97,01 \%$ & $96,95 \%$ & $97,28 \%$ & $96,68 \%$ & $96,96 \%$ & $96,89 \%$ \\
\hline \multirow{6}{*}{ 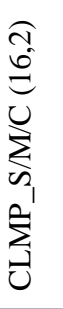 } & \multirow{6}{*}{ (j) } & HW & $94,18 \%$ & 0,018 & $91,08 \%$ & $92,08 \%$ & $94,65 \%$ & $96,62 \%$ & $96,50 \%$ & $95,21 \%$ & $92,36 \%$ & $94,96 \%$ \\
\hline & & NN & $89,79 \%$ & 0,022 & $86,97 \%$ & $93,40 \%$ & $91,01 \%$ & $88,55 \%$ & $88,60 \%$ & $89,39 \%$ & $93,13 \%$ & $87,29 \%$ \\
\hline & & $\mathrm{LN}$ & $82,49 \%$ & 0,017 & $80,10 \%$ & $83,15 \%$ & $84,82 \%$ & $81,35 \%$ & $82,00 \%$ & $84,84 \%$ & $83,49 \%$ & $80,17 \%$ \\
\hline & & $\mathrm{CB}$ & $96,24 \%$ & 0,003 & $95,86 \%$ & $96,93 \%$ & $96,29 \%$ & $96,36 \%$ & $96,56 \%$ & $96,10 \%$ & $95,88 \%$ & $95,90 \%$ \\
\hline & & SP & $97,32 \%$ & 0,003 & $96,79 \%$ & $97,80 \%$ & $97,38 \%$ & $97,42 \%$ & $97,58 \%$ & $97,14 \%$ & $97,63 \%$ & $96,78 \%$ \\
\hline & & LZ & $97,49 \%$ & 0,004 & $96,96 \%$ & $98,07 \%$ & $97,61 \%$ & $97,75 \%$ & $97,73 \%$ & $97,26 \%$ & $97,77 \%$ & $96,75 \%$ \\
\hline \multirow{6}{*}{ 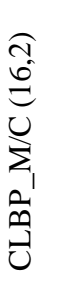 } & \multirow{6}{*}{ ల్ల } & HW & $86,13 \%$ & 0,017 & $83,28 \%$ & $82,93 \%$ & $86,43 \%$ & $87,42 \%$ & $87,52 \%$ & $87,70 \%$ & $86,52 \%$ & $87,23 \%$ \\
\hline & & NN & $80,06 \%$ & 0,044 & $74,22 \%$ & $87,01 \%$ & $78,06 \%$ & $81,67 \%$ & $80,86 \%$ & $77,43 \%$ & $86,85 \%$ & $74,41 \%$ \\
\hline & & $\mathrm{LN}$ & $74,05 \%$ & 0,009 & $73,10 \%$ & $73,52 \%$ & $75,78 \%$ & $73,78 \%$ & $73,90 \%$ & $75,40 \%$ & $74,07 \%$ & $72,85 \%$ \\
\hline & & CB & $84,31 \%$ & 0,004 & $84,16 \%$ & $84,91 \%$ & $83,96 \%$ & $84,69 \%$ & $84,46 \%$ & $83,86 \%$ & $84,84 \%$ & $83,61 \%$ \\
\hline & & SP & $87,00 \%$ & 0,003 & $86,82 \%$ & $87,24 \%$ & $87,16 \%$ & $87,28 \%$ & $87,34 \%$ & $86,77 \%$ & $87,20 \%$ & $86,20 \%$ \\
\hline & & LZ & $87,90 \%$ & 0,004 & $87,76 \%$ & $88,29 \%$ & $87,60 \%$ & $88,25 \%$ & $88,23 \%$ & $87,71 \%$ & $88,29 \%$ & $87,04 \%$ \\
\hline
\end{tabular}




\begin{tabular}{|c|c|c|c|c|c|c|c|c|c|c|c|c|}
\hline & Dim. & & Média & $\begin{array}{l}\text { Desvio } \\
\text { padrão }\end{array}$ & $40^{\circ}$ & $80^{\circ}$ & $120^{\circ}$ & $160^{\circ}$ & $200^{\circ}$ & $240^{\circ}$ & $280^{\circ}$ & $320^{\circ}$ \\
\hline \multirow{6}{*}{ 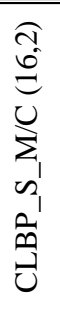 } & & & & 0,018 & & $87,71 \%$ & $91,66 \%$ & $92,42 \%$ & $92,97 \%$ & $92,39 \%$ & $90,21 \%$ & $91,04 \%$ \\
\hline & & $\mathrm{NN}$ & & 068 & & & & & $77,95 \%$ & & $39,76 \%$ & $59,48 \%$ \\
\hline & & $\mathrm{LN}$ & $51,67 \%$ & 0,021 & $49,12 \%$ & $51,26 \%$ & & & & & & $49,42 \%$ \\
\hline & & $\mathrm{CB}$ & $84,75 \%$ & 0,008 & $83,29 \%$ & $85,83 \%$ & $84,78 \%$ & $85,26 \%$ & $84,46 \%$ & $84,97 \%$ & $85,58 \%$ & $83,84 \%$ \\
\hline & & SP & $92,67 \%$ & 0,004 & $91,80 \%$ & $92,99 \%$ & $92,79 \%$ & $92,97 \%$ & $92,84 \%$ & $92,68 \%$ & $93,18 \%$ & $92,11 \%$ \\
\hline & & $\mathrm{LZ}$ & & 0003 & $93,73 \%$ & $94,63 \%$ & $94,34 \%$ & & & & & $94,07 \%$ \\
\hline \multirow{6}{*}{ 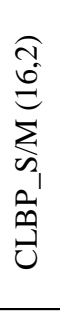 } & \multirow{6}{*}{$\underset{\sim}{\sim}$} & & & & & & & & & & & \\
\hline & & $\mathrm{NN}$ & $67,42 \%$ & 0,083 & $56,90 \%$ & $81,14 \%$ & $65,20 \%$ & $66,95 \%$ & $67,24 \%$ & & & $56,81 \%$ \\
\hline & & $\mathrm{LN}$ & & 0,026 & & & & & & & & \\
\hline & & $\mathrm{CB}$ & $87,48 \%$ & 0,010 & $85,45 \%$ & $88,72 \%$ & & & & & & $85,88 \%$ \\
\hline & & SP & & & & & & & & & & \\
\hline & & $\mathrm{LZ}$ & & 0,002 & & & & & & & & \\
\hline \multirow{6}{*}{ 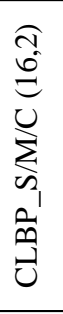 } & \multirow{6}{*}{$\stackrel{d}{0}^{\infty}$} & & & & & & & & & & & \\
\hline & & $\mathrm{NN}$ & & 0,046 & & & & & & & & \\
\hline & & $\mathrm{LN}$ & $68,20 \%$ & 0,029 & $64,48 \%$ & $68,46 \%$ & $72,80 \%$ & & & & & \\
\hline & & $\mathrm{CB}$ & & 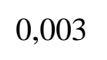 & & & & & & & & $94,44 \%$ \\
\hline & & SP & $97,37 \%$ & 0,002 & $97,05 \%$ & $97,50 \%$ & $97,53 \%$ & $97,37 \%$ & $97,48 \%$ & $97,46 \%$ & $97,44 \%$ & $97,15 \%$ \\
\hline & & $\mathrm{LZ}$ & & 0,001 & & & & & & & & \\
\hline \multirow{6}{*}{ 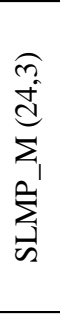 } & \multirow{6}{*}{$\stackrel{\Delta}{I}$} & & & & & & & & & & & \\
\hline & & $\mathrm{NN}$ & & 0,019 & & & & & & & & $89,32 \%$ \\
\hline & & $\mathrm{LN}$ & & & & & & & & & & \\
\hline & & $\mathrm{CB}$ & & 0,003 & & & & & & & & \\
\hline & & SP & & & & & & & & & & \\
\hline & & & & & & & & & & & & \\
\hline \multirow{6}{*}{ 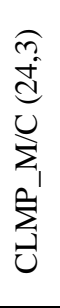 } & \multirow{6}{*}{ กี } & & & & & & & & & & & \\
\hline & & $\mathrm{NN}$ & & & & & & & & & & \\
\hline & & $\mathrm{LN}$ & & & & & & & & & & \\
\hline & & $\mathrm{CB}$ & & 0,003 & $88,20 \%$ & $88,54 \%$ & & & & & & $87,72 \%$ \\
\hline & & SP & & & & $88,60 \%$ & $88,44 \%$ & & & & & $88,29 \%$ \\
\hline & & $\mathrm{LZ}$ & & & & & & & & & & \\
\hline \multirow{6}{*}{ 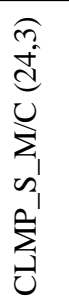 } & \multirow{6}{*}{$\stackrel{\infty}{\infty}$} & & & & & & & & & & & $96,52 \%$ \\
\hline & & $\mathrm{NN}$ & & 0,00 & & & & & & & & \\
\hline & & $\mathrm{LN}$ & & 0,003 & $93,71 \%$ & $94,11 \%$ & $94,16 \%$ & $93,66 \%$ & $94,01 \%$ & & $94,46 \%$ & $93,63 \%$ \\
\hline & & $\mathrm{CB}$ & $97,08 \%$ & 0,001 & $97,16 \%$ & $97,20 \%$ & $96,97 \%$ & $97,23 \%$ & $97,08 \%$ & $96,89 \%$ & $97,03 \%$ & $97,08 \%$ \\
\hline & & SP & & 0,001 & $97,44 \%$ & $97,61 \%$ & & $97,50 \%$ & $97,40 \%$ & $97,27 \%$ & $97,28 \%$ & $97,38 \%$ \\
\hline & & $\mathrm{LZ}$ & & 0,002 & $97,56 \%$ & $97,62 \%$ & $97,16 \%$ & $97,56 \%$ & $97,36 \%$ & $97,20 \%$ & $97,13 \%$ & $97,34 \%$ \\
\hline
\end{tabular}




\begin{tabular}{|c|c|c|c|c|c|c|c|c|c|c|c|c|}
\hline & Dim & & Média & $\begin{array}{l}\text { Desvio } \\
\text { padrão }\end{array}$ & $40^{\circ}$ & $80^{\circ}$ & $120^{\circ}$ & $160^{\circ}$ & $200^{\circ}$ & $240^{\circ}$ & $280^{\circ}$ & $320^{\circ}$ \\
\hline \multirow{6}{*}{ 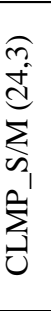 } & \multirow{6}{*}{$\frac{0}{6}$} & & & 0,016 & & & & & & & & $95,49 \%$ \\
\hline & & $\mathrm{NN}$ & $9484 \%$ & 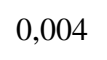 & $95,58 \%$ & $94,43 \%$ & $94,54 \%$ & $94,70 \%$ & $94,59 \%$ & $4,74 \%$ & $94,60 \%$ & $5,56 \%$ \\
\hline & & $\mathrm{LN}$ & & 0,008 & & & & & & & & \\
\hline & & $\mathrm{CB}$ & $96,22 \%$ & 0,001 & $96,17 \%$ & $96,46 \%$ & $96,05 \%$ & $96,24 \%$ & $96,34 \%$ & $96,29 \%$ & $96,26 \%$ & $95,98 \%$ \\
\hline & & SP & $96,75 \%$ & 0,002 & & & & & & & & \\
\hline & & LZ & &, 002 & & & & & & & & $96,45 \%$ \\
\hline \multirow{6}{*}{ 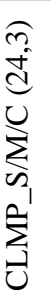 } & \multirow{6}{*}{$\stackrel{\sim}{\approx}$} & & & & & & & & & & & \\
\hline & & $\mathrm{NN}$ & $95,95 \%$ & 0,003 & $96,66 \%$ & $6,08 \%$ & $96,08 \%$ & $95,76 \%$ & & & & \\
\hline & & $\mathrm{LN}$ & & & & & & & & & & \\
\hline & & $\mathrm{CB}$ & & 0,002 & & & & & & & & \\
\hline & & SP & $96,66 \%$ & 0,002 & $96,71 \%$ & & $96,68 \%$ & & & & & $96,27 \%$ \\
\hline & & $\mathrm{LZ}$ & & & & & & & & & & \\
\hline \multirow{6}{*}{ 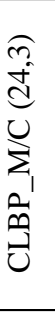 } & \multirow{6}{*}{ ก } & & & & & & & & & & & \\
\hline & & & & & & & & & & & & \\
\hline & & $\mathrm{LN}$ & & 0,011 & & & & & & & & $74,36 \%$ \\
\hline & & $\mathrm{CB}$ & & 0,003 & & & & & & & & \\
\hline & & SP & & & & & & & & & & \\
\hline & & & & דפחת & $88,34 \%$ & $88,18 \%$ & $25 \%$ & & & & & $40 \%$ \\
\hline \multirow{6}{*}{ 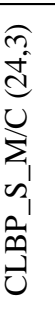 } & \multirow{6}{*}{$\stackrel{\infty}{\infty}$} & & & & & & & & & & & \\
\hline & & & & & & & & & & & & \\
\hline & & $\mathrm{LN}$ & & & & & & & & & & $62,06 \%$ \\
\hline & & $\mathrm{CB}$ & $90,16 \%$ & 0,005 & $89,09 \%$ & $90,75 \%$ & $90,16 \%$ & & $90,03 \%$ & & $96 \%$ & $89,68 \%$ \\
\hline & & SP & & & & & & & & & & \\
\hline & & $\mathrm{LZ}$ & & & & & & & & & & \\
\hline \multirow{6}{*}{ 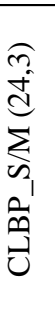 } & \multirow{6}{*}{$\frac{0}{6}$} & & & & & & & & & & & \\
\hline & & $\mathrm{NN}$ & & 0,020 & $84,17 \%$ & & & & $86,40 \%$ & & & \\
\hline & & $\mathrm{LN}$ & & & & & & & & & & $98 \%$ \\
\hline & & $\mathrm{CB}$ & & & & & & & & & & \\
\hline & & SP & & & & & & & & & & $96,62 \%$ \\
\hline & & & & & & & & & & & & \\
\hline \multirow{6}{*}{ 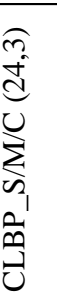 } & \multirow{6}{*}{ 芳 } & & & & & & & & & & & \\
\hline & & $\mathrm{NN}$ & & & & $94,06 \%$ & & & & & & $91,14 \%$ \\
\hline & & $\mathrm{LN}$ & & & & & & & & & & \\
\hline & & $\mathrm{CB}$ & $96,36 \%$ & 0,002 & $96,05 \%$ & $96,62 \%$ & $96,49 \%$ & $96,48 \%$ & $96,27 \%$ & $96,24 \%$ & $96,44 \%$ & $96,25 \%$ \\
\hline & & SP & & & & & & & & & & $91,49 \%$ \\
\hline & & $\mathrm{LZ}$ & $97,59 \%$ & 0,001 & $97,48 \%$ & $97,65 \%$ & $97,66 \%$ & $97,72 \%$ & $97,69 \%$ & $97,52 \%$ & $97,49 \%$ & $97,51 \%$ \\
\hline
\end{tabular}




\section{APÊNDICE C - Acurácias médias obtidas pelos descritores avaliados no Experimento 3}

\begin{tabular}{|c|c|c|c|c|c|c|c|c|c|c|c|c|}
\hline \multicolumn{13}{|c|}{$70 \times 70$ pixels } \\
\hline & Dim. & & Média & $\begin{array}{l}\text { Desvio } \\
\text { padrão }\end{array}$ & $40^{\circ}$ & $80^{\circ}$ & $120^{\circ}$ & $160^{\circ}$ & $200^{\circ}$ & $240^{\circ}$ & $280^{\circ}$ & $320^{\circ}$ \\
\hline \multirow{6}{*}{ 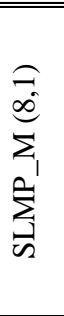 } & \multirow{6}{*}{$\bar{\nabla}$} & $\mathrm{HW}$ & $86,86 \%$ & 0,019 & $85,46 \%$ & $85,01 \%$ & $85,97 \%$ & $88,88 \%$ & $89,60 \%$ & $88,00 \%$ & $83,46 \%$ & $88,48 \%$ \\
\hline & & $\mathrm{NN}$ & $44,78 \%$ & 0,176 & $28,34 \%$ & $74,57 \%$ & $30,91 \%$ & $44,80 \%$ & $45,71 \%$ & $30,78 \%$ & $75,44 \%$ & $27,69 \%$ \\
\hline & & $\mathrm{LN}$ & $33,40 \%$ & 0,022 & $31,00 \%$ & $33,77 \%$ & $37,36 \%$ & $32,21 \%$ & $31,78 \%$ & $36,84 \%$ & $33,47 \%$ & $30,76 \%$ \\
\hline & & $\mathrm{CB}$ & $69,84 \%$ & 0,007 & $68,32 \%$ & $70,09 \%$ & $70,25 \%$ & $69,33 \%$ & $70,12 \%$ & $70,32 \%$ & $70,98 \%$ & $69,28 \%$ \\
\hline & & SP & $85,48 \%$ & 0,010 & $83,74 \%$ & $86,53 \%$ & $84,95 \%$ & $86,26 \%$ & $86,03 \%$ & $85,03 \%$ & $87,10 \%$ & $84,17 \%$ \\
\hline & & LZ & $88,61 \%$ & 0,011 & $86,98 \%$ & $89,80 \%$ & $88,13 \%$ & $89,04 \%$ & $89,18 \%$ & $87,84 \%$ & $90,42 \%$ & $87,49 \%$ \\
\hline \multirow{6}{*}{ 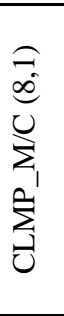 } & \multirow{6}{*}{ ㄱ. } & $\mathrm{HW}$ & $72,44 \%$ & 0,016 & $71,69 \%$ & $70,73 \%$ & $73,69 \%$ & $72,71 \%$ & $73,00 \%$ & $73,59 \%$ & $69,28 \%$ & $74,86 \%$ \\
\hline & & NN & $66,07 \%$ & 0,058 & $59,18 \%$ & $74,84 \%$ & $61,41 \%$ & $68,34 \%$ & $69,16 \%$ & $61,81 \%$ & $74,59 \%$ & $59,23 \%$ \\
\hline & & $\mathrm{LN}$ & $65,47 \%$ & 0,005 & $64,61 \%$ & $65,21 \%$ & $66,12 \%$ & $65,41 \%$ & $65,90 \%$ & $66,33 \%$ & $65,20 \%$ & $64,97 \%$ \\
\hline & & $\mathrm{CB}$ & $73,10 \%$ & 0,003 & $72,80 \%$ & $73,09 \%$ & $73,04 \%$ & $73,57 \%$ & $73,56 \%$ & $73,25 \%$ & $72,86 \%$ & $72,61 \%$ \\
\hline & & SP & $75,21 \%$ & 0,003 & $75,35 \%$ & $75,45 \%$ & $74,80 \%$ & $75,40 \%$ & $75,70 \%$ & $75,16 \%$ & $75,33 \%$ & $74,52 \%$ \\
\hline & & LZ & $75,52 \%$ & 0,005 & $75,91 \%$ & $76,03 \%$ & $75,09 \%$ & $75,59 \%$ & $76,11 \%$ & $74,45 \%$ & $75,95 \%$ & $75,02 \%$ \\
\hline \multirow{6}{*}{ 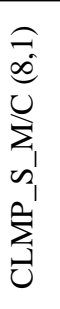 } & \multirow{6}{*}{ i } & HW & $84,58 \%$ & 0,011 & $82,92 \%$ & $82,59 \%$ & $85,68 \%$ & $85,32 \%$ & $85,15 \%$ & $83,96 \%$ & $84,88 \%$ & $86,13 \%$ \\
\hline & & $\mathrm{NN}$ & $75,73 \%$ & 0,069 & $67,98 \%$ & $85,69 \%$ & $70,15 \%$ & $79,08 \%$ & $80,23 \%$ & $69,91 \%$ & $85,78 \%$ & $66,99 \%$ \\
\hline & & $\mathrm{LN}$ & $62,13 \%$ & 0,011 & $60,95 \%$ & $62,33 \%$ & $63,98 \%$ & $61,28 \%$ & $60,99 \%$ & $64,07 \%$ & $62,27 \%$ & $61,20 \%$ \\
\hline & & $\mathrm{CB}$ & $77,78 \%$ & 0,005 & $77,27 \%$ & $78,71 \%$ & $77,05 \%$ & $77,72 \%$ & $78,14 \%$ & $77,78 \%$ & $78,41 \%$ & $77,13 \%$ \\
\hline & & SP & $83,94 \%$ & 0,005 & $83,69 \%$ & $84,63 \%$ & $83,64 \%$ & $83,88 \%$ & $84,32 \%$ & $83,68 \%$ & $84,72 \%$ & $82,97 \%$ \\
\hline & & LZ & $85,06 \%$ & 0,005 & $85,01 \%$ & $85,74 \%$ & $84,53 \%$ & $85,04 \%$ & $85,52 \%$ & $84,98 \%$ & $85,49 \%$ & $84,14 \%$ \\
\hline \multirow{6}{*}{ 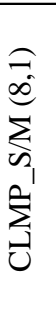 } & \multirow{6}{*}{ 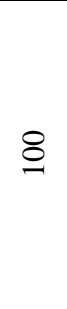 } & HW & $83,22 \%$ & 0,018 & $81,29 \%$ & $80,73 \%$ & $84,73 \%$ & $84,91 \%$ & $84,79 \%$ & $84,95 \%$ & $80,22 \%$ & $84,11 \%$ \\
\hline & & $\mathrm{NN}$ & $40,29 \%$ & 0,157 & $25,04 \%$ & $67,51 \%$ & $28,22 \%$ & $40,69 \%$ & $41,04 \%$ & $27,44 \%$ & $67,07 \%$ & $25,32 \%$ \\
\hline & & $\mathrm{LN}$ & $31,29 \%$ & 0,017 & $30,41 \%$ & $29,97 \%$ & $34,24 \%$ & $31,58 \%$ & $30,48 \%$ & $34,15 \%$ & $29,00 \%$ & $30,49 \%$ \\
\hline & & $\mathrm{CB}$ & $61,71 \%$ & 0,005 & $60,82 \%$ & $61,88 \%$ & $61,82 \%$ & $61,74 \%$ & $62,30 \%$ & $61,55 \%$ & $62,47 \%$ & $61,12 \%$ \\
\hline & & SP & $78,04 \%$ & 0,008 & $76,72 \%$ & $78,90 \%$ & $77,96 \%$ & $78,34 \%$ & $78,70 \%$ & $77,70 \%$ & $79,27 \%$ & $76,76 \%$ \\
\hline & & LZ & $81,40 \%$ & 0,007 & $80,48 \%$ & $82,29 \%$ & $81,11 \%$ & $81,69 \%$ & $81,86 \%$ & $80,93 \%$ & $82,56 \%$ & $80,29 \%$ \\
\hline \multirow{6}{*}{ 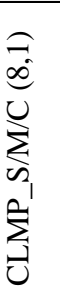 } & \multirow{6}{*}{ ¿ั } & HW & $84,67 \%$ & 0,016 & $83,26 \%$ & $81,46 \%$ & $84,92 \%$ & $85,36 \%$ & $86,14 \%$ & $85,51 \%$ & $83,61 \%$ & $87,10 \%$ \\
\hline & & $\mathrm{NN}$ & $53,03 \%$ & 0,153 & $38,84 \%$ & $78,89 \%$ & $42,69 \%$ & $54,71 \%$ & $54,69 \%$ & $41,64 \%$ & $78,28 \%$ & $34,47 \%$ \\
\hline & & $\mathrm{LN}$ & $38,81 \%$ & 0,014 & $37,39 \%$ & $38,46 \%$ & $41,50 \%$ & $37,79 \%$ & $38,41 \%$ & $41,01 \%$ & $38,18 \%$ & $37,73 \%$ \\
\hline & & $\mathrm{CB}$ & $65,90 \%$ & 0,005 & $65,23 \%$ & $66,65 \%$ & $66,41 \%$ & $65,62 \%$ & $66,02 \%$ & $65,77 \%$ & $66,45 \%$ & $65,01 \%$ \\
\hline & & SP & $80,28 \%$ & 0,005 & $79,19 \%$ & $81,16 \%$ & $80,44 \%$ & $80,34 \%$ & $80,54 \%$ & $80,17 \%$ & $80,77 \%$ & $79,64 \%$ \\
\hline & & $\mathrm{LZ}$ & $83,58 \%$ & 0,007 & $82,09 \%$ & $84,54 \%$ & $83,39 \%$ & $83,60 \%$ & $84,10 \%$ & $83,70 \%$ & $84,18 \%$ & $83,04 \%$ \\
\hline \multirow{6}{*}{ 己े } & \multirow{6}{*}{ กิ } & HW & $68,00 \%$ & 0,009 & $66,48 \%$ & $67,77 \%$ & $68,56 \%$ & $69,40 \%$ & $68,97 \%$ & $67,76 \%$ & $66,66 \%$ & $68,41 \%$ \\
\hline & & $\mathrm{NN}$ & $60,74 \%$ & 0,069 & $53,76 \%$ & $72,11 \%$ & $55,25 \%$ & $63,26 \%$ & $62,73 \%$ & $54,46 \%$ & $71,26 \%$ & $53,12 \%$ \\
\hline & & $\mathrm{LN}$ & $52,39 \%$ & 0,010 & $51,86 \%$ & $51,65 \%$ & $53,93 \%$ & $52,00 \%$ & $51,31 \%$ & $54,17 \%$ & $52,60 \%$ & $51,62 \%$ \\
\hline & & $\mathrm{CB}$ & $64,02 \%$ & 0,007 & $63,41 \%$ & $65,29 \%$ & $63,03 \%$ & $64,48 \%$ & $63,80 \%$ & $63,65 \%$ & $64,98 \%$ & $63,49 \%$ \\
\hline & & SP & $68,95 \%$ & 0,013 & $68,45 \%$ & $71,36 \%$ & $67,59 \%$ & $68,86 \%$ & $68,87 \%$ & $67,87 \%$ & $70,87 \%$ & $67,74 \%$ \\
\hline & & LZ & $70,24 \%$ & 0,014 & $69,53 \%$ & $73,12 \%$ & $69,04 \%$ & $70,03 \%$ & $70,00 \%$ & $68,76 \%$ & $72,12 \%$ & $69,30 \%$ \\
\hline
\end{tabular}




\begin{tabular}{|c|c|c|c|c|c|c|c|c|c|c|c|c|}
\hline \multicolumn{13}{|c|}{$70 \times 70$ pixels } \\
\hline \multicolumn{3}{|c|}{ Dim. } & \multirow{2}{*}{$\begin{array}{c}\text { Média } \\
79,46 \%\end{array}$} & \multirow{2}{*}{$\begin{array}{c}\begin{array}{c}\text { Desvio } \\
\text { padrão }\end{array} \\
0,01\end{array}$} & \multirow{2}{*}{$\begin{array}{c}40^{\circ} \\
77,93 \%\end{array}$} & \multirow{2}{*}{$\begin{array}{c}80^{\circ} \\
78,41 \%\end{array}$} & \multirow{2}{*}{$\begin{array}{c}120^{\circ} \\
79,86 \%\end{array}$} & \multirow{2}{*}{$\begin{array}{c}160^{\circ} \\
80,55 \%\end{array}$} & \multirow{2}{*}{$\begin{array}{c}200^{\circ} \\
80,31 \%\end{array}$} & \multirow{2}{*}{$\begin{array}{c}240^{\circ} \\
80,16 \%\end{array}$} & \multirow{2}{*}{$\frac{280^{\circ}}{78,15 \%}$} & \multirow{2}{*}{$\begin{array}{c}320^{\circ} \\
80,33 \%\end{array}$} \\
\hline 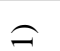 & & HW & & & & & & & & & & \\
\hline$\alpha$ & \multirow{5}{*}{ లి } & $\mathrm{NN}$ & $54,52 \%$ & 0,141 & & $64,84 \%$ & $69,31 \%$ & & & & & $44,44 \%$ \\
\hline$\sum$ & & $\mathrm{LN}$ & $30,81 \%$ & 0,015 & $29,74 \%$ & $30,60 \%$ & $33,55 \%$ & $29,62 \%$ & $29,10 \%$ & $33,40 \%$ & $30,48 \%$ & $29,96 \%$ \\
\hline$\Omega_{1}^{1}$ & & $\mathrm{CB}$ & $58,75 \%$ & 0,008 & $59,56 \%$ & $59,58 \%$ & $58,00 \%$ & $57,68 \%$ & $57,66 \%$ & & $59,87 \%$ & $59,51 \%$ \\
\hline 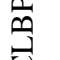 & & SP & $73,78 \%$ & 0,013 & $74,37 \%$ & $75,98 \%$ & $72,66 \%$ & $73,14 \%$ & $72,21 \%$ & $72,32 \%$ & $75,48 \%$ & $74,04 \%$ \\
\hline & & $\mathrm{LZ}$ & $77,63 \%$ & 0,013 & & $80,08 \%$ & $76,30 \%$ & & & & & $77,68 \%$ \\
\hline \multirow{6}{*}{ 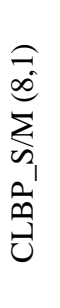 } & \multirow{6}{*}{8} & HW & 78480 & 0017 & $76,31 \%$ & $77,43 \%$ & $77,84 \%$ & $80,85 \%$ & $81,07 \%$ & & $75,86 \%$ & \\
\hline & & NN & $79,45 \%$ & 0,013 & $78,35 \%$ & $82,00 \%$ & $78,76 \%$ & $79,67 \%$ & $78,84 \%$ & $7 \%$ & $1 \%$ & $3 \%$ \\
\hline & & $\mathrm{LN}$ & $24,49 \%$ & 0,009 & $23,91 \%$ & $23,64 \%$ & $25,84 \%$ & & & & & \\
\hline & & CB & $54,60 \%$ & 0,005 & $54,60 \%$ & $55,04 \%$ & $53,89 \%$ & $54,99 \%$ & $54,78 \%$ & $53,76 \%$ & $55,27 \%$ & $54,45 \%$ \\
\hline & & SP & & 0,014 & & $74,85 \%$ & & & & & & \\
\hline & & $\mathrm{LZ}$ & & & & & & & & & & \\
\hline \multirow{6}{*}{ 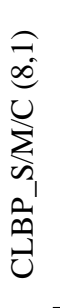 } & \multirow{6}{*}{ 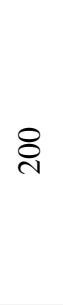 } & & & & & & & & & & & \\
\hline & & $\mathrm{NN}$ & $76,41 \%$ & 0,020 & & $80,18 \%$ & $74,63 \%$ & $76,61 \%$ & & & & \\
\hline & & $\mathrm{LN}$ & $41,58 \%$ & $0,0<0$ & $40,30 \%$ & $40,55 \%$ & $45,15 \%$ & $40,91 \%$ & $40,03 \%$ & $7 \%$ & $5 \%$ & $31 \%$ \\
\hline & & $\mathrm{CB}$ & $71,31 \%$ & 0,003 & $71,00 \%$ & $71,65 \%$ & $71,39 \%$ & $71,01 \%$ & $70,98 \%$ & $71,55 \%$ & $71,81 \%$ & $71,09 \%$ \\
\hline & & SP & & & & & & & & & & \\
\hline & & $\mathrm{LZ}$ & $85,91 \%$ & 0,006 & & $86,76 \%$ & $85,52 \%$ & $86,04 \%$ & $85,72 \%$ & & & \\
\hline \multirow{6}{*}{ 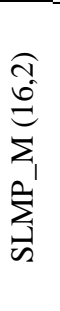 } & \multirow{6}{*}{ તิ } & & & $0,0<1$ & & $78,56 \%$ & & & & & & \\
\hline & & NN & $68,62 \%$ & 0,031 & $65,80 \%$ & $73,41 \%$ & $70,19 \%$ & $66,16 \%$ & $65,79 \%$ & $68,74 \%$ & $73,79 \%$ & $65,09 \%$ \\
\hline & & $\mathrm{LN}$ & $60,56 \%$ & 0,017 & $58,57 \%$ & $59,59 \%$ & $63,41 \%$ & $60,14 \%$ & $60,42 \%$ & $63,50 \%$ & $60,00 \%$ & $58,81 \%$ \\
\hline & & $\mathrm{CB}$ & $82,26 \%$ & 0,006 & $81,03 \%$ & $82,63 \%$ & $82,15 \%$ & $82,60 \%$ & $82,62 \%$ & & & $50 \%$ \\
\hline & & $\mathrm{SP}$ & $85,94 \%$ & 0,006 & $85,09 \%$ & $86,61 \%$ & $85,46 \%$ & $86,57 \%$ & $86,27 \%$ & $86,00 \%$ & $86,56 \%$ & $84,94 \%$ \\
\hline & & $\mathrm{LZ}$ & $86,49 \%$ & 0,000 & & $87,28 \%$ & $86,29 \%$ & & & & & $85,60 \%$ \\
\hline \multirow{6}{*}{ 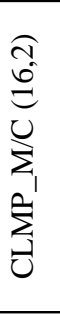 } & \multirow{6}{*}{ లి } & HW & & & & & & & & & $73,37 \%$ & $75,30 \%$ \\
\hline & & NN & & & & & & & & & & \\
\hline & & $\mathrm{LN}$ & $70,18 \%$ & 0,006 & & $70,88 \%$ & & & & & & $69,30 \%$ \\
\hline & & $\mathrm{CB}$ & $74,79 \%$ & 0,004 & $74,85 \%$ & $75,25 \%$ & $74,10 \%$ & $74,40 \%$ & & & & \\
\hline & & SP & $75,77 \%$ & 0,003 & $76,00 \%$ & $76,11 \%$ & $75,64 \%$ & & & & $75,11 \%$ & $75,72 \%$ \\
\hline & & $\mathrm{LZ}$ & & 0004 & & $76,30 \%$ & $75,38 \%$ & $76,13 \%$ & $76,29 \%$ & $75,75 \%$ & $75,29 \%$ & $75,76 \%$ \\
\hline \multirow{6}{*}{ 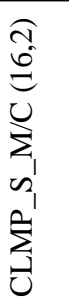 } & \multirow{6}{*}{ in } & HW & $84,91 \%$ & 0,010 & $82,40 \%$ & $82,33 \%$ & $86,49 \%$ & $86,38 \%$ & $86,34 \%$ & & $84,15 \%$ & $86,12 \%$ \\
\hline & & $\mathrm{NN}$ & $78,04 \%$ & 0,018 & $76,73 \%$ & $80,86 \%$ & $79,46 \%$ & $76,72 \%$ & $75,91 \%$ & $77,67 \%$ & $80,69 \%$ & $76,31 \%$ \\
\hline & & $\mathrm{LN}$ & $77,58 \%$ & 0,011 & $75,88 \%$ & $77,96 \%$ & $79,06 \%$ & $77,15 \%$ & $77,24 \%$ & $79,40 \%$ & $77,57 \%$ & $76,36 \%$ \\
\hline & & $\mathrm{CB}$ & $87,72 \%$ & 0,002 & $87,21 \%$ & $87,77 \%$ & $88,12 \%$ & $87,83 \%$ & $87,54 \%$ & $87,94 \%$ & $87,53 \%$ & $87,81 \%$ \\
\hline & & SP & $88,73 \%$ & 0,002 & $88,46 \%$ & $88,92 \%$ & $89,03 \%$ & $88,90 \%$ & $88,38 \%$ & $88,90 \%$ & $88,52 \%$ & $88,73 \%$ \\
\hline & & $\mathrm{LZ}$ & $88,59 \%$ & 0,002 & $88,35 \%$ & $88,85 \%$ & $88,84 \%$ & $88,82 \%$ & $88,26 \%$ & $88,66 \%$ & $88,36 \%$ & $88,61 \%$ \\
\hline
\end{tabular}




\begin{tabular}{|c|c|c|c|c|c|c|c|c|c|c|c|c|}
\hline \multicolumn{13}{|c|}{$70 \times 70$ pixels } \\
\hline \multicolumn{3}{|c|}{ Dim. } & \multirow{2}{*}{$\begin{array}{c}\text { Média } \\
83,63 \%\end{array}$} & \multirow{2}{*}{$\begin{array}{c}\begin{array}{l}\text { Desvio } \\
\text { padrão }\end{array} \\
0,021\end{array}$} & \multirow{2}{*}{$\begin{array}{c}40^{\circ} \\
81,10 \%\end{array}$} & \multirow{2}{*}{$\frac{80^{\circ}}{81,62 \%}$} & \multirow{2}{*}{$\frac{120^{\circ}}{85,40 \%}$} & \multirow{2}{*}{$\frac{160^{\circ}}{86,07 \%}$} & \multirow{2}{*}{$\frac{200^{\circ}}{85,19 \%}$} & \multirow{2}{*}{$\frac{240^{\circ}}{85,14 \%}$} & \multirow{2}{*}{ 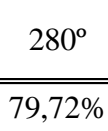 } & \multirow{2}{*}{$\frac{320^{\circ}}{84,82 \%}$} \\
\hline & \multirow{6}{*}{$\underset{\sim}{\stackrel{\sim}{n}}$} & HW & & & & & & & & & & \\
\hline$\underbrace{\circ}$ & & NN & $77,96 \%$ & 0,023 & $75,61 \%$ & $81,40 \%$ & $79,36 \%$ & $76,18 \%$ & $76,21 \%$ & $77,52 \%$ & $82,04 \%$ & $75,37 \%$ \\
\hline$\sum$ & & $\mathrm{LN}$ & $68,53 \%$ & 0,017 & $66,15 \%$ & $69,37 \%$ & $70,82 \%$ & $66,71 \%$ & $68,03 \%$ & $71,44 \%$ & $68,57 \%$ & $67,13 \%$ \\
\hline$e_{1}$ & & $\mathrm{CB}$ & $84,18 \%$ & 0,004 & $83,28 \%$ & $84,63 \%$ & $83,86 \%$ & $84,29 \%$ & $84,40 \%$ & $84,33 \%$ & $84,76 \%$ & $83,88 \%$ \\
\hline$z$ & & SP & $86,92 \%$ & 0,003 & $86,24 \%$ & $87,42 \%$ & $86,72 \%$ & $87,08 \%$ & $87,11 \%$ & $86,92 \%$ & $87,04 \%$ & $86,86 \%$ \\
\hline & & LZ & $87,53 \%$ & 0,003 & $87,12 \%$ & $87,85 \%$ & $87,44 \%$ & $87,54 \%$ & $88,01 \%$ & $87,32 \%$ & $87,70 \%$ & $87,28 \%$ \\
\hline \multirow{6}{*}{ 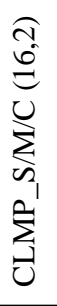 } & \multirow{6}{*}{$\stackrel{0}{J}^{\infty}$} & HW & $82,10 \%$ & 0,016 & $79,67 \%$ & $80,06 \%$ & $83,16 \%$ & $84,07 \%$ & $83,99 \%$ & $82,47 \%$ & $80,38 \%$ & $82,98 \%$ \\
\hline & & NN & $78,11 \%$ & 0,019 & $76,17 \%$ & $81,33 \%$ & $78,67 \%$ & $77,42 \%$ & $77,31 \%$ & $77,03 \%$ & $81,26 \%$ & $75,66 \%$ \\
\hline & & $\mathrm{LN}$ & $69,66 \%$ & 0,013 & $67,40 \%$ & $70,08 \%$ & $71,04 \%$ & $69,33 \%$ & $69,29 \%$ & $72,03 \%$ & $70,01 \%$ & $68,09 \%$ \\
\hline & & CB & $82,77 \%$ & 0,004 & $81,86 \%$ & $83,32 \%$ & $82,63 \%$ & $83,05 \%$ & $83,20 \%$ & $82,53 \%$ & $82,90 \%$ & $82,66 \%$ \\
\hline & & SP & $84,82 \%$ & 0,005 & $83,88 \%$ & $85,30 \%$ & $85,03 \%$ & $84,80 \%$ & $85,46 \%$ & $84,90 \%$ & $84,94 \%$ & $84,24 \%$ \\
\hline & & $\mathrm{LZ}$ & $85,36 \%$ & 0,004 & $84,41 \%$ & $85,98 \%$ & $85,49 \%$ & $85,64 \%$ & $85,58 \%$ & $85,43 \%$ & $85,22 \%$ & $85,16 \%$ \\
\hline \multirow{6}{*}{ 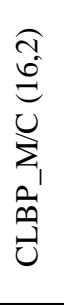 } & \multirow{6}{*}{ r } & HW & $72,72 \%$ & 0,012 & $70,91 \%$ & $70,28 \%$ & $73,83 \%$ & $73,09 \%$ & $73,68 \%$ & $73,54 \%$ & $72,81 \%$ & $73,61 \%$ \\
\hline & & NN & $67,80 \%$ & 0,033 & $63,47 \%$ & $73,29 \%$ & $67,19 \%$ & $68,72 \%$ & $68,15 \%$ & $65,72 \%$ & $72,66 \%$ & $63,16 \%$ \\
\hline & & $\mathrm{LN}$ & $63,63 \%$ & 0,007 & $62,78 \%$ & $63,19 \%$ & $64,74 \%$ & $64,05 \%$ & $63,57 \%$ & $64,80 \%$ & $63,04 \%$ & $62,88 \%$ \\
\hline & & $\mathrm{CB}$ & $71,51 \%$ & 0,004 & $70,77 \%$ & $71,44 \%$ & $72,29 \%$ & $72,01 \%$ & $71,86 \%$ & $71,08 \%$ & $71,25 \%$ & $71,39 \%$ \\
\hline & & SP & $73,82 \%$ & 0,004 & $73,04 \%$ & $73,96 \%$ & $74,26 \%$ & $74,50 \%$ & $74,19 \%$ & $73,48 \%$ & $73,64 \%$ & $73,45 \%$ \\
\hline & & $\mathrm{LZ}$ & $74,41 \%$ & 0,004 & $73,99 \%$ & $74,43 \%$ & $74,90 \%$ & $75,04 \%$ & $74,84 \%$ & $73,93 \%$ & $74,35 \%$ & $73,83 \%$ \\
\hline \multirow{6}{*}{ 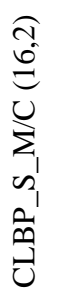 } & \multirow{6}{*}{ in } & HW & $81,91 \%$ & 0,019 & $79,29 \%$ & $79,57 \%$ & $83,71 \%$ & $8311 \%$ & $84,19 \%$ & $83,56 \%$ & $79,42 \%$ & $82,44 \%$ \\
\hline & & NN & $42,79 \%$ & 0,131 & $44,31 \%$ & $32,68 \%$ & $64,91 \%$ & $29,26 \%$ & $44,31 \%$ & $32,68 \%$ & $64,91 \%$ & $29,26 \%$ \\
\hline & & $\mathrm{LN}$ & $50,09 \%$ & 0,020 & $47,08 \%$ & $50,12 \%$ & $53,48 \%$ & $49,36 \%$ & $49,04 \%$ & $53,27 \%$ & $49,95 \%$ & $48,38 \%$ \\
\hline & & $\mathrm{CB}$ & $77,75 \%$ & 0,008 & $75,78 \%$ & $78,36 \%$ & $77,64 \%$ & $78,22 \%$ & $77,70 \%$ & $78,26 \%$ & $78,49 \%$ & $77,54 \%$ \\
\hline & & SP & $85,09 \%$ & 0,005 & $84,25 \%$ & $85,75 \%$ & $84,79 \%$ & $85,34 \%$ & $84,80 \%$ & $85,61 \%$ & $85,50 \%$ & $84,66 \%$ \\
\hline & & $\mathrm{LZ}$ & $86,61 \%$ & 0,003 & $86,38 \%$ & $87,11 \%$ & $86,50 \%$ & $86,87 \%$ & $86,11 \%$ & & & $86,22 \%$ \\
\hline \multirow{6}{*}{ 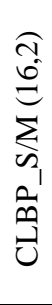 } & \multirow{6}{*}{$\underset{\sim}{\stackrel{\Xi}{\sim}}$} & HW & $81,42 \%$ & 0,027 & $80,70 \%$ & $78,25 \%$ & $82,59 \%$ & $83,74 \%$ & $83,53 \%$ & $83,68 \%$ & $75,67 \%$ & $83,23 \%$ \\
\hline & & NN & $27,88 \%$ & 0,161 & $14,05 \%$ & $56,56 \%$ & $16,07 \%$ & $25,46 \%$ & $25,84 \%$ & & & $13,22 \%$ \\
\hline & & $\mathrm{LN}$ & $48,09 \%$ & 0,028 & $43,63 \%$ & $48,28 \%$ & $51,99 \%$ & $47,09 \%$ & $46,97 \%$ & $52,78 \%$ & $49,17 \%$ & $44,77 \%$ \\
\hline & & $\mathrm{CB}$ & $81,34 \%$ & 0,011 & $78,86 \%$ & $82,33 \%$ & $81,18 \%$ & $81,76 \%$ & $81,75 \%$ & $81,66 \%$ & $82,85 \%$ & $80,34 \%$ \\
\hline & & SP & $88,28 \%$ & 0,006 & $87,09 \%$ & $89,05 \%$ & $87,94 \%$ & $88,72 \%$ & $88,22 \%$ & $88,63 \%$ & $89,03 \%$ & $87,52 \%$ \\
\hline & & $\mathrm{LZ}$ & $89,57 \%$ & 0,005 & $88,76 \%$ & $90,03 \%$ & $89,30 \%$ & $90,18 \%$ & $89,57 \%$ & $89,57 \%$ & $90,15 \%$ & $88,96 \%$ \\
\hline \multirow{6}{*}{ 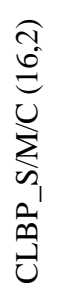 } & \multirow{6}{*}{$\stackrel{\infty}{0}$} & HW & $86,56 \%$ & 0,019 & $84,89 \%$ & $84,64 \%$ & $88,20 \%$ & $88,09 \%$ & $88,15 \%$ & $88,31 \%$ & $82,59 \%$ & $87,59 \%$ \\
\hline & & NN & $46,72 \%$ & 0,165 & $31,81 \%$ & $75,37 \%$ & $35,25 \%$ & $45,46 \%$ & $45,68 \%$ & $33,81 \%$ & $75,71 \%$ & $30,63 \%$ \\
\hline & & $\mathrm{LN}$ & $64,51 \%$ & 0,025 & $60,84 \%$ & $64,24 \%$ & $68,28 \%$ & $63,39 \%$ & $63,37 \%$ & $68,48 \%$ & $65,82 \%$ & $61,62 \%$ \\
\hline & & CB & $87,46 \%$ & 0,005 & $86,41 \%$ & $87,81 \%$ & $87,34 \%$ & $87,70 \%$ & $87,57 \%$ & $87,49 \%$ & $88,43 \%$ & $86,95 \%$ \\
\hline & & SP & $90,74 \%$ & 0,003 & $90,24 \%$ & $91,16 \%$ & $90,46 \%$ & $90,88 \%$ & $90,83 \%$ & $91,12 \%$ & $91,03 \%$ & $90,19 \%$ \\
\hline & & $\mathrm{LZ}$ & $91,38 \%$ & 0,002 & $91,23 \%$ & $91,72 \%$ & $91,17 \%$ & $91,57 \%$ & $91,32 \%$ & $91,60 \%$ & $91,51 \%$ & $90,88 \%$ \\
\hline
\end{tabular}




\begin{tabular}{|c|c|c|c|c|c|c|c|c|c|c|c|c|}
\hline \multicolumn{13}{|c|}{70 x 70 pixels } \\
\hline & Dim. & & Média & $\begin{array}{l}\text { Desvio } \\
\text { padrão }\end{array}$ & $40^{\circ}$ & $80^{\circ}$ & $120^{\circ}$ & $160^{\circ}$ & $200^{\circ}$ & $240^{\circ}$ & $280^{\circ}$ & $320^{\circ}$ \\
\hline \multirow{6}{*}{ 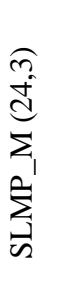 } & & HW & $78,16 \%$ & 0,018 & $75,56 \%$ & $76,42 \%$ & $79,58 \%$ & $79,65 \%$ & $79,95 \%$ & $79,24 \%$ & $75,09 \%$ & $79,81 \%$ \\
\hline & & NN & $73,42 \%$ & 0,011 & $75,44 \%$ & $72,50 \%$ & $72,53 \%$ & $73,15 \%$ & $72,59 \%$ & $72,92 \%$ & $72,73 \%$ & $75,50 \%$ \\
\hline & & $\mathrm{LN}$ & $65,89 \%$ & 0,012 & $64,32 \%$ & $66,08 \%$ & $67,85 \%$ & $64,36 \%$ & $65,52 \%$ & $67,69 \%$ & $66,08 \%$ & $65,25 \%$ \\
\hline & & $\mathrm{CB}$ & $79,28 \%$ & 0,004 & $78,30 \%$ & $79,72 \%$ & $79,40 \%$ & $79,36 \%$ & $79,30 \%$ & $79,50 \%$ & $79,48 \%$ & $79,16 \%$ \\
\hline & & SP & $80,95 \%$ & 0,004 & $80,03 \%$ & $81,26 \%$ & $81,44 \%$ & $81,01 \%$ & $81,02 \%$ & $81,15 \%$ & $80,72 \%$ & $81,00 \%$ \\
\hline & & $\mathrm{LZ}$ & & 0,004 & $80,47 \%$ & $81,59 \%$ & $81,67 \%$ & & $81,41 \%$ & & $81,05 \%$ & $81,20 \%$ \\
\hline \multirow{6}{*}{ 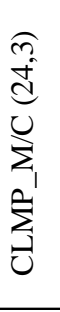 } & \multirow{6}{*}{ กิ } & HW & $69,93 \%$ & 0,012 & $68,27 \%$ & $67,42 \%$ & $71,06 \%$ & $69,88 \%$ & $71,14 \%$ & $70,71 \%$ & $70,14 \%$ & $70,79 \%$ \\
\hline & & NN & $69,90 \%$ & 0,004 & $69,26 \%$ & $70,61 \%$ & $69,52 \%$ & $69,82 \%$ & $69,84 \%$ & $70,06 \%$ & $70,42 \%$ & $69,63 \%$ \\
\hline & & $\mathrm{LN}$ & $67,36 \%$ & 0,005 & $66,30 \%$ & $67,60 \%$ & 67,5 & & & & & $38 \%$ \\
\hline & & $\mathrm{CB}$ & $70,50 \%$ & 0,004 & $69,62 \%$ & $70,84 \%$ & $70,44 \%$ & $70,79 \%$ & $70,41 \%$ & $70,71 \%$ & $70,33 \%$ & $70,86 \%$ \\
\hline & & SP & $70,87 \%$ & 0,003 & $70,35 \%$ & $71,44 \%$ & $70,88 \%$ & $71,14 \%$ & $70,60 \%$ & $71,07 \%$ & $70,41 \%$ & $71,06 \%$ \\
\hline & & $\mathrm{LZ}$ & $71,07 \%$ & 0,004 & $70,63 \%$ & $71,69 \%$ & $71,15 \%$ & $71,16 \%$ & $70,72 \%$ & $71,41 \%$ & $2 \%$ & $71,27 \%$ \\
\hline \multirow{6}{*}{ 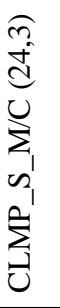 } & \multirow{6}{*}{$\stackrel{\infty}{\sim}$} & HW & $83,27 \%$ & 0,014 & $01,11 \%$ & $81,19 \%$ & $85,12 \%$ & 84,0 & $84,44 \%$ & $83,73 \%$ & $8 \%$ & 84, \\
\hline & & NN & $81,38 \%$ & 0,005 & $82,22 \%$ & $81,21 \%$ & $81,00 \%$ & & & & & \\
\hline & & $\mathrm{LN}$ & $81,99 \%$ & 0,005 & $80,90 \%$ & $82,10 \%$ & $82,30 \%$ & $81,48 \%$ & $82,02 \%$ & $82,68 \%$ & $82,56 \%$ & $81,87 \%$ \\
\hline & & $\mathrm{CB}$ & $85,48 \%$ & 0,002 & $85,16 \%$ & $85,65 \%$ & $85,53 \%$ & $85,52 \%$ & $85,28 \%$ & $85,45 \%$ & $85,46 \%$ & $85,81 \%$ \\
\hline & & SP & $85,79 \%$ & 0,002 & $85,53 \%$ & $86,11 \%$ & $85,66 \%$ & $85,64 \%$ & $85,81 \%$ & $85,98 \%$ & & $85,94 \%$ \\
\hline & & $\mathrm{LZ}$ & $85,68 \%$ & 0,003 & $85,36 \%$ & $86,22 \%$ & $85,65 \%$ & & $85,41 \%$ & & $3 \%$ & $96 \%$ \\
\hline \multirow{6}{*}{ 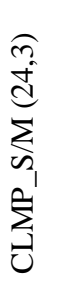 } & \multirow{6}{*}{$\frac{1}{6}$} & HW & & 0,012 & & $79,82 \%$ & & & & & $78,65 \%$ & \\
\hline & & NN & $81,18 \%$ & 0,004 & $81,26 \%$ & $81,40 \%$ & $80,59 \%$ & $80,89 \%$ & $81,45 \%$ & $80,75 \%$ & & $82,16 \%$ \\
\hline & & $\mathrm{LN}$ & $77,83 \%$ & 0,006 & $76,82 \%$ & $78,32 \%$ & $78,24 \%$ & $77,24 \%$ & $77,63 \%$ & $78,88 \%$ & $78,25 \%$ & $77,22 \%$ \\
\hline & & $\mathrm{CB}$ & $82,42 \%$ & 0,003 & $81,95 \%$ & $82,35 \%$ & $82,05 \%$ & $82,53 \%$ & $82,66 \%$ & $82,36 \%$ & $1 \%$ & $82,73 \%$ \\
\hline & & SP & $83,16 \%$ & 0,003 & $82,95 \%$ & $82,96 \%$ & $83,00 \%$ & $83,02 \%$ & $83,63 \%$ & $82,89 \%$ & $83,21 \%$ & $83,60 \%$ \\
\hline & & $\mathrm{LZ}$ & & 0,003 & & $82,96 \%$ & & & & & & \\
\hline \multirow{6}{*}{ 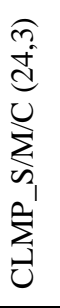 } & \multirow{6}{*}{ ๙̃ } & HW & $78,97 \%$ & & & $77,47 \%$ & $80,08 \%$ & & & & & $79,59 \%$ \\
\hline & & NN & & 0,004 & & $78,85 \%$ & & & & & & \\
\hline & & $\mathrm{LN}$ & $75,24 \%$ & 0,004 & $74,57 \%$ & $75,57 \%$ & $75,71 \%$ & $74,90 \%$ & $75,46 \%$ & $75,80 \%$ & $75,20 \%$ & $74,72 \%$ \\
\hline & & $\mathrm{CB}$ & $79,29 \%$ & 0,002 & $79,14 \%$ & $79,17 \%$ & $79,01 \%$ & $79,29 \%$ & $79,53 \%$ & $79,34 \%$ & $79,55 \%$ & $79,25 \%$ \\
\hline & & SP & $80,25 \%$ & 0,003 & $80,19 \%$ & $80,29 \%$ & $79,89 \%$ & $79,92 \%$ & $80,48 \%$ & $80,42 \%$ & & $80,92 \%$ \\
\hline & & LZ & $80,29 \%$ & 0,003 & $80,18 \%$ & $80,17 \%$ & $79,82 \%$ & $80,52 \%$ & $80,60 \%$ & $80,76 \%$ & $79,93 \%$ & $80,35 \%$ \\
\hline \multirow{6}{*}{ 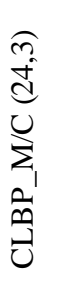 } & \multirow{6}{*}{ กิ } & HW & $69,19 \%$ & 0,008 & $68,86 \%$ & $67,06 \%$ & $69,64 \%$ & 07,2010 & $69,92 \%$ & $69,48 \%$ & $69,52 \%$ & $69,76 \%$ \\
\hline & & NN & $66,29 \%$ & 0,019 & $64,46 \%$ & $69,65 \%$ & $66,08 \%$ & $66,45 \%$ & $39 \%$ & $64,84 \%$ & $69,31 \%$ & $63,64 \%$ \\
\hline & & $\mathrm{LN}$ & $56,99 \%$ & 0,203 & $63,93 \%$ & $65,19 \%$ & $66,27 \%$ & $64,99 \%$ & $65,34 \%$ & $65,70 \%$ & $65,30 \%$ & $64,89 \%$ \\
\hline & & $\mathrm{CB}$ & $70,51 \%$ & 0,004 & $69,70 \%$ & $70,48 \%$ & $70,60 \%$ & $71,28 \%$ & $70,78 \%$ & $70,27 \%$ & $70,42 \%$ & $70,57 \%$ \\
\hline & & SP & $71,84 \%$ & 0,002 & $71,52 \%$ & $71,82 \%$ & $71,94 \%$ & & $72,10 \%$ & $71,56 \%$ & $71,79 \%$ & $71,87 \%$ \\
\hline & & $\mathrm{LZ}$ & $72,07 \%$ & 0,003 & $71,58 \%$ & $72,37 \%$ & $72,34 \%$ & $72,36 \%$ & $72,22 \%$ & $71,79 \%$ & $71,66 \%$ & $72,25 \%$ \\
\hline
\end{tabular}




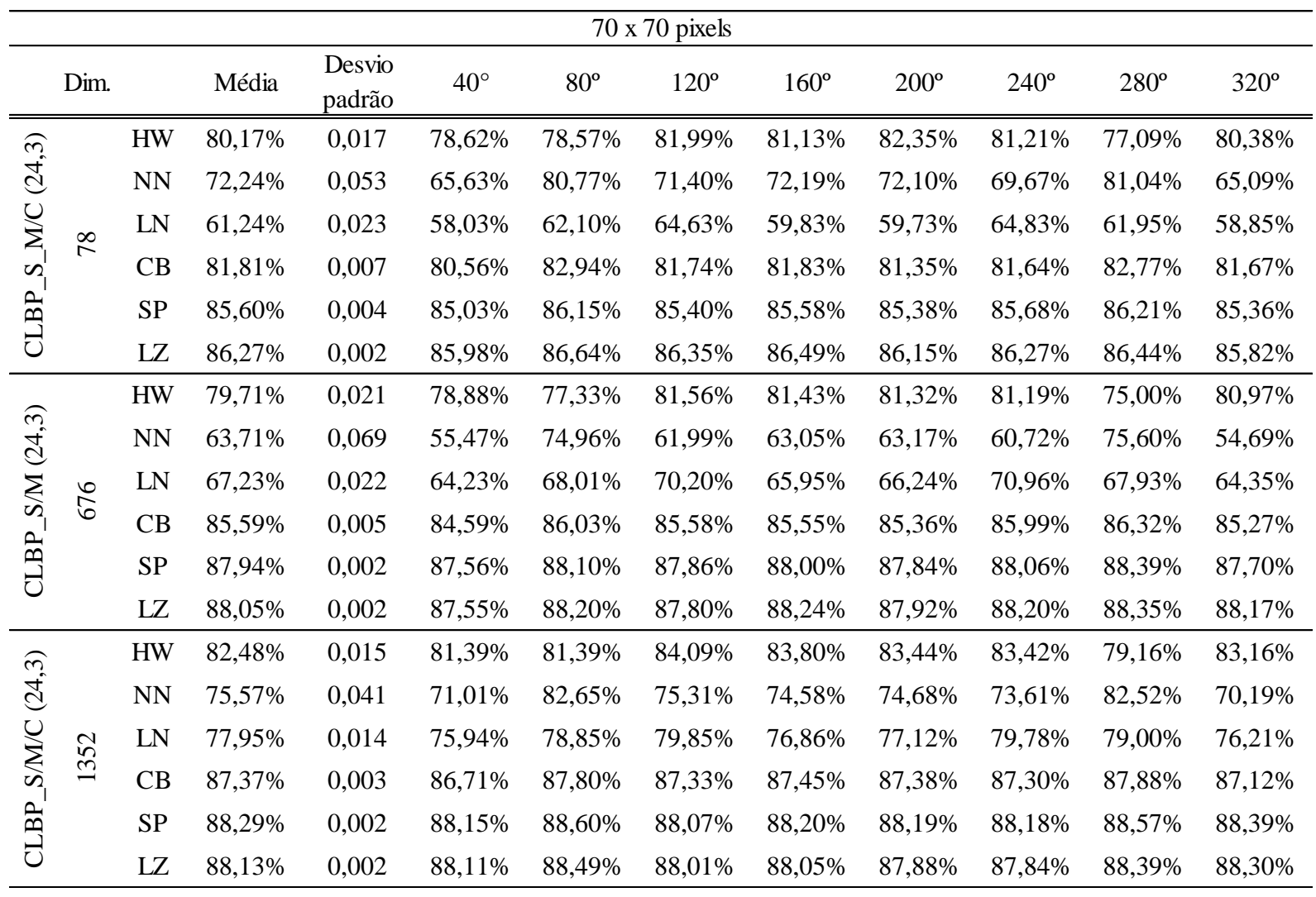




\begin{tabular}{|c|c|c|c|c|c|c|c|c|c|c|c|c|}
\hline \multicolumn{13}{|c|}{$90 \times 90$ pixels } \\
\hline & Dim. & & Média & $\begin{array}{l}\text { Desvio } \\
\text { padrão }\end{array}$ & $40^{\circ}$ & $80^{\circ}$ & $120^{\circ}$ & $160^{\circ}$ & $200^{\circ}$ & $240^{\circ}$ & $280^{\circ}$ & $320^{\circ}$ \\
\hline \multirow{6}{*}{ 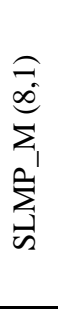 } & \multirow{6}{*}{$\bar{\nabla}$} & HW & $89,52 \%$ & 0018 & $88,09 \%$ & $87,64 \%$ & $88,52 \%$ & $91,53 \%$ & $92,06 \%$ & $90,44 \%$ & $86,65 \%$ & $91,21 \%$ \\
\hline & & NN & $73,52 \%$ & 0,009 & $72,24 \%$ & $74,08 \%$ & $74,16 \%$ & $73,20 \%$ & $73,78 \%$ & $73,76 \%$ & $74,87 \%$ & $72,06 \%$ \\
\hline & & $\mathrm{LN}$ & $34,94 \%$ & 0,025 & $32,32 \%$ & $35,14 \%$ & $39,47 \%$ & $33,44 \%$ & $33,30 \%$ & $38,92 \%$ & $34,94 \%$ & $32,00 \%$ \\
\hline & & $\mathrm{CB}$ & $73,52 \%$ & 0,009 & $72,24 \%$ & $74,08 \%$ & $74,16 \%$ & $73,20 \%$ & $73,78 \%$ & $73,76 \%$ & $74,87 \%$ & $72,06 \%$ \\
\hline & & SP & $89,34 \%$ & 0,011 & $87,61 \%$ & $90,22 \%$ & $89,36 \%$ & $89,98 \%$ & $89,56 \%$ & $88,94 \%$ & $91,17 \%$ & $87,84 \%$ \\
\hline & & $\mathrm{LZ}$ & $92,19 \%$ & 0,010 & $90,63 \%$ & $93,13 \%$ & $91,92 \%$ & $92,98 \%$ & $92,77 \%$ & $91,65 \%$ & & $90,72 \%$ \\
\hline \multirow{6}{*}{ 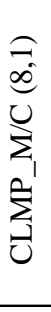 } & \multirow{6}{*}{ ๙ి } & HW & $79,87 \%$ & 0,031 & $80,31 \%$ & $78,03 \%$ & & & & & & $82,77 \%$ \\
\hline & & $\mathrm{NN}$ & $80,12 \%$ & 0,003 & $80,19 \%$ & $80,42 \%$ & $80,08 \%$ & $79,77 \%$ & $80,11 \%$ & $80,44 \%$ & $80,59 \%$ & $79,38 \%$ \\
\hline & & $\mathrm{LN}$ & $70,86 \%$ & 0,007 & $69,87 \%$ & $70,80 \%$ & $71,93 \%$ & $70,76 \%$ & $70,73 \%$ & $72,04 \%$ & $70,73 \%$ & $70,01 \%$ \\
\hline & & $\mathrm{CB}$ & $80,12 \%$ & 0,003 & $80,19 \%$ & $80,42 \%$ & $80,08 \%$ & $79,77 \%$ & $80,11 \%$ & $80,44 \%$ & $80,59 \%$ & $79,38 \%$ \\
\hline & & SP & $83,29 \%$ & 0,003 & $83,22 \%$ & $82,97 \%$ & $83,03 \%$ & $83,62 \%$ & $83,64 \%$ & $83,32 \%$ & $83,80 \%$ & $82,72 \%$ \\
\hline & & $\mathrm{LZ}$ & $84,05 \%$ & 0,003 & $83,81 \%$ & $83,87 \%$ & $83,60 \%$ & $84,19 \%$ & $83,90 \%$ & $84,13 \%$ & $84,43 \%$ & $84,48 \%$ \\
\hline \multirow{6}{*}{ 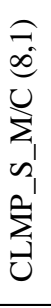 } & \multirow{6}{*}{ ஜे } & & סדירנט & 0,012 & $88,25 \%$ & $87,93 \%$ & $90,40 \%$ & $90,73 \%$ & $90,86 \%$ & $89,64 \%$ & $89,91 \%$ & $91,83 \%$ \\
\hline & & NN & $83,59 \%$ & 0,009 & $83,11 \%$ & $85,20 \%$ & $82,65 \%$ & $83,65 \%$ & $83,13 \%$ & $84,07 \%$ & $84,59 \%$ & $82,28 \%$ \\
\hline & & $\mathrm{LN}$ & $66,36 \%$ & 0,014 & & $67,16 \%$ & & & & & & $64,98 \%$ \\
\hline & & $\mathrm{CB}$ & $83,59 \%$ & 0,009 & $83,11 \%$ & $85,20 \%$ & $82,65 \%$ & $83,65 \%$ & $83,13 \%$ & $84,07 \%$ & $84,59 \%$ & $82,28 \%$ \\
\hline & & SP & $90,31 \%$ & 0,006 & $90,04 \%$ & $91,35 \%$ & $89,98 \%$ & $90,33 \%$ & $90,16 \%$ & $90,24 \%$ & $91,15 \%$ & $89,24 \%$ \\
\hline & & $\mathrm{LZ}$ & $91,18 \%$ & 0,006 & $91,04 \%$ & $92,17 \%$ & $90,81 \%$ & & & & & $89,94 \%$ \\
\hline \multirow{6}{*}{ 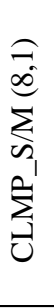 } & \multirow{6}{*}{8} & HW & $88,07 \%$ & 0,018 & $86,70 \%$ & $85,28 \%$ & $89,47 \%$ & $89,65 \%$ & $89,55 \%$ & $89,87 \%$ & $85,15 \%$ & $88,85 \%$ \\
\hline & & NN & $66,41 \%$ & 0,006 & $65,53 \%$ & $66,67 \%$ & $66,65 \%$ & $66,56 \%$ & $66,44 \%$ & $66,68 \%$ & $67,30 \%$ & $65,44 \%$ \\
\hline & & $\mathrm{LN}$ & $32,71 \%$ & 0,019 & $31,78 \%$ & $31,58 \%$ & $35,96 \%$ & $32,60 \%$ & $31,40 \%$ & $35,96 \%$ & $30,35 \%$ & $32,06 \%$ \\
\hline & & CB & & 0,006 & & $66,67 \%$ & $66,65 \%$ & $66,56 \%$ & $66,44 \%$ & & $67,30 \%$ & $65,44 \%$ \\
\hline & & SP & & 0,008 & & $84,36 \%$ & & & & & & $82,18 \%$ \\
\hline & & $\mathrm{LZ}$ & $87,07 \%$ & 0007 & $86,03 \%$ & $87,85 \%$ & $86,68 \%$ & $87,28 \%$ & & $87,16 \%$ & $88,22 \%$ & $85,80 \%$ \\
\hline \multirow{6}{*}{ 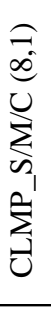 } & \multirow{6}{*}{ ¿্ণ } & HW & $89,96 \%$ & 0,017 & $88,56 \%$ & $86,30 \%$ & $90,36 \%$ & $90,89 \%$ & $91,72 \%$ & $91,09 \%$ & $89,01 \%$ & $91,75 \%$ \\
\hline & & NN & $72,79 \%$ & 0,008 & $71,37 \%$ & $74,04 \%$ & $73,34 \%$ & $72,84 \%$ & $72,80 \%$ & $72,76 \%$ & $73,70 \%$ & $71,48 \%$ \\
\hline & & $\mathrm{LN}$ & $41,53 \%$ & 0,016 & $39,76 \%$ & $41,46 \%$ & $44,56 \%$ & $40,62 \%$ & $40,57 \%$ & $43,97 \%$ & $41,59 \%$ & $39,68 \%$ \\
\hline & & $\mathrm{CB}$ & $72,79 \%$ & 0,008 & $71,37 \%$ & $74,04 \%$ & $73,34 \%$ & $72,84 \%$ & $72,80 \%$ & $72,76 \%$ & $73,70 \%$ & $71,48 \%$ \\
\hline & & SP & $87,87 \%$ & 0,007 & $86,87 \%$ & $88,86 \%$ & $87,76 \%$ & $88,42 \%$ & $87,68 \%$ & $87,74 \%$ & $88,92 \%$ & $86,74 \%$ \\
\hline & & $\mathrm{LZ}$ & $90,57 \%$ & 0,006 & $89,74 \%$ & $91,28 \%$ & $90,18 \%$ & $90,99 \%$ & $90,74 \%$ & $90,31 \%$ & $91,46 \%$ & $89,88 \%$ \\
\hline \multirow{6}{*}{ 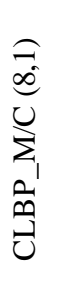 } & \multirow{6}{*}{ શิ } & HW & $74,21 \%$ & 0,010 & $73,07 \%$ & $73,89 \%$ & $74,60 \%$ & $75,67 \%$ & $75,07 \%$ & $73,46 \%$ & $72,45 \%$ & $75,48 \%$ \\
\hline & & NN & $69,36 \%$ & 0,010 & $69,22 \%$ & $71,07 \%$ & & $69,09 \%$ & $69,11 \%$ & $68,78 \%$ & $71,26 \%$ & $68,43 \%$ \\
\hline & & $\mathrm{LN}$ & $56,06 \%$ & 0,012 & $55,52 \%$ & $55,86 \%$ & $57,59 \%$ & $55,12 \%$ & $54,76 \%$ & $58,26 \%$ & $56,71 \%$ & $54,63 \%$ \\
\hline & & $\mathrm{CB}$ & $69,36 \%$ & 0,010 & $69,22 \%$ & $71,07 \%$ & $67,94 \%$ & $69,09 \%$ & $69,11 \%$ & $68,78 \%$ & $71,26 \%$ & $68,43 \%$ \\
\hline & & SP & $74,74 \%$ & 0,014 & $74,25 \%$ & $77,26 \%$ & $73,68 \%$ & $74,59 \%$ & $74,24 \%$ & $73,64 \%$ & $77,16 \%$ & $73,06 \%$ \\
\hline & & $\mathrm{LZ}$ & $76,40 \%$ & 0,015 & $75,75 \%$ & $79,00 \%$ & $75,14 \%$ & $75,89 \%$ & $76,25 \%$ & $75,40 \%$ & $78,96 \%$ & $74,78 \%$ \\
\hline
\end{tabular}




\begin{tabular}{|c|c|c|c|c|c|c|c|c|c|c|c|c|}
\hline \multicolumn{13}{|c|}{$90 \times 90$ pixels } \\
\hline \multicolumn{3}{|c|}{ Dim. } & \multirow{2}{*}{$\begin{array}{c}\text { Média } \\
84,01 \%\end{array}$} & \multirow{2}{*}{$\begin{array}{c}\text { Desvio } \\
\text { padrão }\end{array}$} & \multirow{2}{*}{$\begin{array}{c}40^{\circ} \\
82,23 \%\end{array}$} & \multirow{2}{*}{$\begin{array}{c}80^{\circ} \\
83,19 \%\end{array}$} & \multirow{2}{*}{$\frac{120^{\circ}}{84,32 \%}$} & \multirow{2}{*}{$\frac{160^{\circ}}{85,51 \%}$} & \multirow{2}{*}{$\frac{200^{\circ}}{84,88 \%}$} & \multirow{2}{*}{$\frac{240^{\circ}}{84,20 \%}$} & \multirow{2}{*}{$\frac{280^{\circ}}{82,00 \%}$} & \multirow{2}{*}{$\frac{320^{\circ}}{85,73 \%}$} \\
\hline ક & & HW & & & & & & & & & & \\
\hline$\infty$ & \multirow{5}{*}{ లి } & NN & $60,58 \%$ & 0,014 & $61,64 \%$ & $62,38 \%$ & $59,23 \%$ & $58,47 \%$ & $59,34 \%$ & $60,11 \%$ & $62,60 \%$ & $60,85 \%$ \\
\hline$\Sigma$ & & $\mathrm{LN}$ & $31,47 \%$ & 0,015 & $30,24 \%$ & $31,69 \%$ & $34,04 \%$ & $30,14 \%$ & $29,71 \%$ & $33,70 \%$ & $31,89 \%$ & $30,32 \%$ \\
\hline$\approx_{1}^{1}$ & & $\mathrm{CB}$ & $60,58 \%$ & 0,014 & $61,64 \%$ & $62,38 \%$ & $59,23 \%$ & $58,47 \%$ & $59,34 \%$ & $60,11 \%$ & $62,60 \%$ & $60,85 \%$ \\
\hline $\overrightarrow{9}$ & & SP & $77,58 \%$ & 0,013 & $77,94 \%$ & $79,95 \%$ & $76,36 \%$ & $76,62 \%$ & $76,33 \%$ & $76,42 \%$ & $79,65 \%$ & $77,33 \%$ \\
\hline & & $\mathrm{LZ}$ & $81,98 \%$ & 0,015 & $82,52 \%$ & $84,62 \%$ & $80,45 \%$ & $80,70 \%$ & $80,65 \%$ & $80,66 \%$ & $84,36 \%$ & $81,84 \%$ \\
\hline \multirow{6}{*}{ 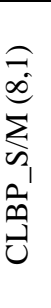 } & \multirow{6}{*}{8} & HW & $82,40 \%$ & 0,019 & $80,28 \%$ & $81,38 \%$ & $81,88 \%$ & $84,53 \%$ & 85,4 & & $79,22 \%$ & $83,05 \%$ \\
\hline & & NN & $55,21 \%$ & 0,006 & $55,04 \%$ & $56,27 \%$ & $54,82 \%$ & $55,40 \%$ & $55,08 \%$ & $54,42 \%$ & $56,10 \%$ & $54,57 \%$ \\
\hline & & $\mathrm{LN}$ & $24,13 \%$ & 0,011 & $23,00 \%$ & $23,24 \%$ & $25,89 \%$ & $25,00 \%$ & $23,93 \%$ & $25,64 \%$ & $22,82 \%$ & $23,51 \%$ \\
\hline & & $\mathrm{CB}$ & $55,21 \%$ & 0,006 & $55,04 \%$ & $56,27 \%$ & $54,82 \%$ & $55,40 \%$ & $55,08 \%$ & $54,42 \%$ & $56,10 \%$ & $54,57 \%$ \\
\hline & & SP & $76,46 \%$ & 0,017 & $74,78 \%$ & $79,24 \%$ & $75,92 \%$ & $76,90 \%$ & $76,90 \%$ & $75,04 \%$ & $79,01 \%$ & $73,91 \%$ \\
\hline & & $\mathrm{LZ}$ & $81,67 \%$ & 0,015 & $79,95 \%$ & $83,89 \%$ & $80,70 \%$ & $82,47 \%$ & $82,35 \%$ & $80,39 \%$ & $84,03 \%$ & $79,61 \%$ \\
\hline \multirow{6}{*}{ 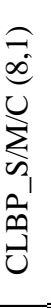 } & \multirow{6}{*}{ 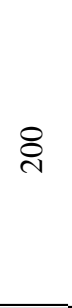 } & & & 0,020 & $90,20 \%$ & $90,06 \%$ & $94,07 \%$ & $94,48 \%$ & $2 \%$ & & & \\
\hline & & NN & $74,70 \%$ & 0,006 & $74,12 \%$ & $75,66 \%$ & $74,85 \%$ & $74,90 \%$ & $74,36 \%$ & $74,77 \%$ & $50 \%$ & $73,42 \%$ \\
\hline & & $\mathrm{LN}$ & $42,05 \%$ & 0,022 & $40,08 \%$ & $40,91 \%$ & $46,51 \%$ & & & & & $40,27 \%$ \\
\hline & & $\mathrm{CB}$ & $74,70 \%$ & 0,006 & $74,12 \%$ & $75,66 \%$ & $74,85 \%$ & $74,90 \%$ & $74,36 \%$ & $74,77 \%$ & $75,50 \%$ & $73,42 \%$ \\
\hline & & SP & $88,44 \%$ & 0,008 & $87,40 \%$ & $89,47 \%$ & $88,66 \%$ & $88,99 \%$ & $88,34 \%$ & $88,45 \%$ & $89,30 \%$ & $86,92 \%$ \\
\hline & & $\mathrm{LZ}$ & $91,13 \%$ & 0,007 & $90,40 \%$ & $91,84 \%$ & $91,28 \%$ & $91,32 \%$ & $91,01 \%$ & $4 \%$ & $4 \%$ & $89,80 \%$ \\
\hline \multirow{6}{*}{ 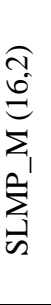 } & \multirow{6}{*}{ సે } & HW & $86,32 \%$ & 0,021 & $83,62 \%$ & $83,92 \%$ & $85,91 \%$ & $88,87 \%$ & $90,18 \%$ & $87,48 \%$ & $2 \%$ & $86,48 \%$ \\
\hline & & NN & $88,60 \%$ & 0,006 & $87,53 \%$ & $88,92 \%$ & $88,13 \%$ & $88,86 \%$ & $88,82 \%$ & $89,07 \%$ & $89,65 \%$ & $87,79 \%$ \\
\hline & & $\mathrm{LN}$ & $64,15 \%$ & 0,019 & $61,32 \%$ & $63,52 \%$ & $67,00 \%$ & $63,32 \%$ & $64,41 \%$ & $67,52 \%$ & $63,92 \%$ & $62,21 \%$ \\
\hline & & CB & $88,60 \%$ & 0,006 & $87,53 \%$ & $88,92 \%$ & $88,13 \%$ & $88,86 \%$ & $88,82 \%$ & $89,07 \%$ & $89,65 \%$ & $87,79 \%$ \\
\hline & & SP & $92,23 \%$ & 0,006 & $91,25 \%$ & $92,58 \%$ & $91,68 \%$ & $92,72 \%$ & $92,73 \%$ & $92,44 \%$ & $93,00 \%$ & $91,43 \%$ \\
\hline & & $\mathrm{LZ}$ & $92,57 \%$ & 0,007 & $91,41 \%$ & $93,13 \%$ & $92,24 \%$ & $93,33 \%$ & $93,08 \%$ & $92,55 \%$ & $93,38 \%$ & $91,45 \%$ \\
\hline \multirow{6}{*}{ 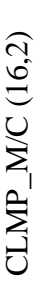 } & \multirow{6}{*}{ లి } & HW & $82,29 \%$ & 0,016 & $80,16 \%$ & $79,03 \%$ & $83,16 \%$ & $82,95 \%$ & $83,39 \%$ & $83,52 \%$ & $82,14 \%$ & $84,00 \%$ \\
\hline & & $\mathrm{NN}$ & $83,33 \%$ & 0,003 & $82,95 \%$ & $83,50 \%$ & $83,19 \%$ & $83,29 \%$ & $83,29 \%$ & $84,00 \%$ & $83,58 \%$ & $82,87 \%$ \\
\hline & & $\mathrm{LN}$ & $77,15 \%$ & 0,008 & $76,13 \%$ & $77,96 \%$ & $77,69 \%$ & $76,25 \%$ & $76,51 \%$ & $78,66 \%$ & $77,68 \%$ & $76,33 \%$ \\
\hline & & $\mathrm{CB}$ & $83,33 \%$ & 0,003 & $82,95 \%$ & $83,50 \%$ & $83,19 \%$ & $83,29 \%$ & $83,29 \%$ & $84,00 \%$ & $83,58 \%$ & $82,87 \%$ \\
\hline & & SP & $84,03 \%$ & 0,005 & $83,86 \%$ & $84,28 \%$ & $84,14 \%$ & & $82,80 \%$ & & & $83,53 \%$ \\
\hline & & $\mathrm{LZ}$ & $84,31 \%$ & 0,003 & $84,54 \%$ & $84,22 \%$ & $84,36 \%$ & $84,36 \%$ & $84,43 \%$ & $84,53 \%$ & $84,54 \%$ & $83,51 \%$ \\
\hline \multirow{6}{*}{ 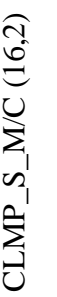 } & \multirow{6}{*}{ in } & HW & $90,26 \%$ & 0,017 & $87,49 \%$ & $87,01 \%$ & $91,21 \%$ & $91,92 \%$ & $91,59 \%$ & $91,20 \%$ & $89,86 \%$ & $91,76 \%$ \\
\hline & & NN & $93,13 \%$ & 0,003 & $92,93 \%$ & $93,36 \%$ & $92,76 \%$ & $92,92 \%$ & $92,90 \%$ & $93,48 \%$ & $93,52 \%$ & $93,18 \%$ \\
\hline & & $\mathrm{LN}$ & $82,33 \%$ & 0,012 & $80,74 \%$ & $82,68 \%$ & $83,67 \%$ & $81,40 \%$ & $81,34 \%$ & $84,64 \%$ & $82,97 \%$ & $81,19 \%$ \\
\hline & & $\mathrm{CB}$ & $93,13 \%$ & 0,003 & $92,93 \%$ & $93,36 \%$ & $92,76 \%$ & $92,92 \%$ & $92,90 \%$ & $93,48 \%$ & $93,52 \%$ & $93,18 \%$ \\
\hline & & SP & $94,14 \%$ & 0,002 & $94,32 \%$ & $94,39 \%$ & $93,88 \%$ & $94,00 \%$ & $93,66 \%$ & $94,36 \%$ & $94,32 \%$ & $94,16 \%$ \\
\hline & & $\mathrm{LZ}$ & $94,08 \%$ & 0,002 & $94,18 \%$ & $94,24 \%$ & $94,00 \%$ & $94,01 \%$ & $93,69 \%$ & $94,17 \%$ & $94,16 \%$ & $94,21 \%$ \\
\hline
\end{tabular}




\begin{tabular}{|c|c|c|c|c|c|c|c|c|c|c|c|c|}
\hline \multicolumn{13}{|c|}{$90 \times 90$ pixels } \\
\hline \multicolumn{3}{|c|}{ Dim. } & Média & $\begin{array}{l}\text { Desvio } \\
\text { padrão }\end{array}$ & $40^{\circ}$ & $80^{\circ}$ & $120^{\circ}$ & $160^{\circ}$ & $200^{\circ}$ & $240^{\circ}$ & $280^{\circ}$ & $320^{\circ}$ \\
\hline \multirow{6}{*}{ 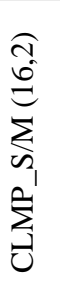 } & \multirow{6}{*}{$\underset{\sim}{\stackrel{d}{n}}$} & HW & $89,70 \%$ & 0,021 & $86,93 \%$ & $87,28 \%$ & $90,70 \%$ & & $91,98 \%$ & $91,27 \%$ & $86,36 \%$ & $90,92 \%$ \\
\hline & & NN & $91,20 \%$ & 0,003 & $90,59 \%$ & $91,40 \%$ & $91,11 \%$ & $91,09 \%$ & $91,14 \%$ & $91,49 \%$ & $91,84 \%$ & $90,93 \%$ \\
\hline & & $\mathrm{LN}$ & $63,78 \%$ & 0,228 & $70,79 \%$ & $73,69 \%$ & $75,, 68 \%$ & $71,36 \%$ & $71,97 \%$ & $76,78 \%$ & $74,33 \%$ & $71,33 \%$ \\
\hline & & $\mathrm{CB}$ & $91,20 \%$ & 0,003 & $90,59 \%$ & $91,40 \%$ & & & & & & \\
\hline & & SP & $93,55 \%$ & 0,003 & $93,21 \%$ & $93,69 \%$ & $93,27 \%$ & $93,76 \%$ & $93,73 \%$ & $94,00 \%$ & $93,82 \%$ & $92,93 \%$ \\
\hline & & $\mathrm{LZ}$ & 93,9 & 0,003 & $93,49 \%$ & $94,11 \%$ & $93,86 \%$ & & & & & \\
\hline \multirow{6}{*}{ 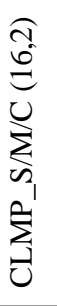 } & \multirow{6}{*}{$\mathfrak{J}^{\infty}$} & HW & $89,52 \%$ & 0,019 & $6,66 \%$ & $87,16 \%$ & $90,64 \%$ & 91, & $91,72 \%$ & 90,7 & $87,36 \%$ & $17 \%$ \\
\hline & & NN & $90,91 \%$ & 0,005 & $90,01 \%$ & $91,43 \%$ & $90,76 \%$ & $91,12 \%$ & $91,04 \%$ & $90,83 \%$ & $91,72 \%$ & $90,37 \%$ \\
\hline & & $\mathrm{LN}$ & $77,25 \%$ & 0,016 & $75,29 \%$ & $77,90 \%$ & $79,40 \%$ & & $76,48 \%$ & & & \\
\hline & & CB & $90,91 \%$ & 0,005 & $90,01 \%$ & $91,43 \%$ & $90,76 \%$ & $91,12 \%$ & $91,04 \%$ & $90,83 \%$ & $91,72 \%$ & $90,37 \%$ \\
\hline & & SP & $92,47 \%$ & 0,006 & $91,34 \%$ & $93,29 \%$ & $92,45 \%$ & $92,65 \%$ & $92,69 \%$ & $92,58 \%$ & $93,13 \%$ & $91,59 \%$ \\
\hline & & $\mathrm{LZ}$ & $92,65 \%$ & 0,005 & $91,79 \%$ & $93,29 \%$ & $92,52 \%$ & $92,97 \%$ & $92,87 \%$ & $92,48 \%$ & $93,34 \%$ & $91,94 \%$ \\
\hline \multirow{6}{*}{ 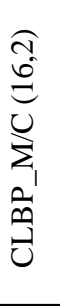 } & \multirow{6}{*}{ ల } & HW & $79,64 \%$ & $0,01 J$ & $77,09 \%$ & $76,89 \%$ & $80,25 \%$ & $80,64 \%$ & $80,85 \%$ & $80,61 \%$ & $79,95 \%$ & $80,82 \%$ \\
\hline & & $\mathrm{NN}$ & $78,32 \%$ & 0,005 & $77,91 \%$ & $78,12 \%$ & $78,64 \%$ & $78,91 \%$ & $78,93 \%$ & $78,03 \%$ & & $77,50 \%$ \\
\hline & & $\mathrm{LN}$ & $69,03 \%$ & 0,009 & & $68,87 \%$ & $70,36 \%$ & & & & & \\
\hline & & $\mathrm{CB}$ & $78,32 \%$ & 0,005 & $77,91 \%$ & $78,12 \%$ & $78,64 \%$ & $78,91 \%$ & $78,93 \%$ & $78,03 \%$ & $78,53 \%$ & $77,50 \%$ \\
\hline & & SP & $80,50 \%$ & 0,005 & $80,06 \%$ & $80,36 \%$ & $80,72 \%$ & $81,00 \%$ & $80,86 \%$ & $80,36 \%$ & & $79,52 \%$ \\
\hline & & $\mathrm{LZ}$ & $80,99 \%$ & 0,005 & & $81,04 \%$ & $81,15 \%$ & $81,35 \%$ & & & & $79,70 \%$ \\
\hline \multirow{6}{*}{ 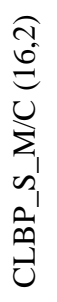 } & \multirow{6}{*}{ in } & HW & & 0,011 & & $84,61 \%$ & & & & & & \\
\hline & & NN & $81,71 \%$ & 0,009 & $79,88 \%$ & $82,56 \%$ & $81,68 \%$ & $82,09 \%$ & $81,60 \%$ & & $5 \%$ & $74 \%$ \\
\hline & & $\mathrm{LN}$ & $51,29 \%$ & 0,022 & $48,15 \%$ & $51,14 \%$ & $55,31 \%$ & $50,37 \%$ & $50,30 \%$ & $54,59 \%$ & $51,70 \%$ & $48,72 \%$ \\
\hline & & $\mathrm{CB}$ & $81,71 \%$ & 0,009 & $79,88 \%$ & $82,56 \%$ & $81,68 \%$ & $82,09 \%$ & $81,60 \%$ & $82,11 \%$ & $83,05 \%$ & $80,74 \%$ \\
\hline & & SP & $89,49 \%$ & 0,004 & $88,69 \%$ & $89,80 \%$ & $89,14 \%$ & $89,77 \%$ & $89,37 \%$ & $89,90 \%$ & $90,19 \%$ & $89,05 \%$ \\
\hline & & $\mathrm{LZ}$ & $90,96 \%$ & & $90,73 \%$ & & $91,34 \%$ & & & & & \\
\hline \multirow{6}{*}{ 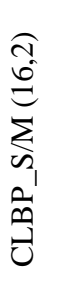 } & \multirow{6}{*}{$\stackrel{\mathbb{N}}{\text { m }}$} & HW & $85,71 \%$ & 0,027 & $84,31 \%$ & $82,96 \%$ & $87,04 \%$ & $88,11 \%$ & $88,20 \%$ & $88,14 \%$ & $79,86 \%$ & $87,05 \%$ \\
\hline & & NN & $85,40 \%$ & 0012 & & $86,75 \%$ & & & & & & \\
\hline & & $\mathrm{LN}$ & $47,41 \%$ & 0,028 & $43,15 \%$ & $47,68 \%$ & $51,77 \%$ & $46,60 \%$ & $46,28 \%$ & $51,77 \%$ & $48,08 \%$ & $43,91 \%$ \\
\hline & & CB & $85,40 \%$ & 0,012 & $83,16 \%$ & $86,75 \%$ & $85,40 \%$ & $86,04 \%$ & $85,61 \%$ & $85,54 \%$ & $87,01 \%$ & $83,72 \%$ \\
\hline & & SP & $92,57 \%$ & 0,005 & $91,60 \%$ & $92,84 \%$ & $92,47 \%$ & $93,07 \%$ & $92,73 \%$ & $92,91 \%$ & $93,14 \%$ & $91,83 \%$ \\
\hline & & $\mathrm{LZ}$ & $93,89 \%$ & 0,005 & $93,06 \%$ & $94,25 \%$ & $93,89 \%$ & $94,68 \%$ & $93,89 \%$ & $94,12 \%$ & $94,19 \%$ & $93,04 \%$ \\
\hline \multirow{6}{*}{ 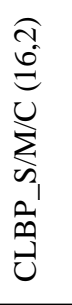 } & \multirow{6}{*}{$\stackrel{0}{J}^{\infty}$} & HW & $91,35 \%$ & $0,0<0$ & $88,77 \%$ & $89,92 \%$ & $93,16 \%$ & $92,96 \%$ & $93,12 \%$ & $93,18 \%$ & $87,51 \%$ & $92,20 \%$ \\
\hline & & NN & $91,74 \%$ & 0,006 & $90,52 \%$ & $92,06 \%$ & $91,44 \%$ & $92,20 \%$ & $91,79 \%$ & $92,28 \%$ & $92,68 \%$ & $90,93 \%$ \\
\hline & & LN & $65,98 \%$ & 0,028 & $61,99 \%$ & $66,20 \%$ & $69,74 \%$ & $64,90 \%$ & $65,00 \%$ & $70,46 \%$ & $67,41 \%$ & $62,14 \%$ \\
\hline & & CB & $91,74 \%$ & 0,006 & $90,52 \%$ & $92,06 \%$ & $91,44 \%$ & $92,20 \%$ & $91,79 \%$ & $92,28 \%$ & $92,68 \%$ & $90,93 \%$ \\
\hline & & SP & $94,96 \%$ & 0,003 & $94,50 \%$ & $95,16 \%$ & $94,84 \%$ & $95,27 \%$ & $94,96 \%$ & $95,14 \%$ & $95,31 \%$ & $94,49 \%$ \\
\hline & & $\mathrm{LZ}$ & $95,55 \%$ & 0,002 & $95,40 \%$ & $95,78 \%$ & $95,42 \%$ & $95,80 \%$ & $95,51 \%$ & $95,64 \%$ & $95,72 \%$ & $95,10 \%$ \\
\hline
\end{tabular}




\begin{tabular}{|c|c|c|c|c|c|c|c|c|c|c|c|c|}
\hline \multicolumn{13}{|c|}{$90 \times 90$ pixels } \\
\hline & Dim. & & Média & $\begin{array}{l}\text { Desvio } \\
\text { padrão }\end{array}$ & $40^{\circ}$ & $80^{\circ}$ & $120^{\circ}$ & $160^{\circ}$ & $200^{\circ}$ & $240^{\circ}$ & $280^{\circ}$ & $320^{\circ}$ \\
\hline \multirow{6}{*}{ 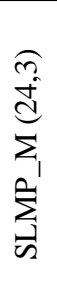 } & \multirow{6}{*}{ I } & HW & $86,02 \%$ & 0,020 & $83,44 \%$ & $83,81 \%$ & $87,24 \%$ & $87,80 \%$ & $87,85 \%$ & $87,84 \%$ & $82,54 \%$ & $87,64 \%$ \\
\hline & & NN & $87,28 \%$ & 0,004 & $86,99 \%$ & $87,87 \%$ & $87,58 \%$ & $87,33 \%$ & $86,76 \%$ & $87,61 \%$ & $87,58 \%$ & $86,50 \%$ \\
\hline & & $\mathrm{LN}$ & $71,35 \%$ & 0,015 & $69,17 \%$ & $71,89 \%$ & $73,78 \%$ & $70,23 \%$ & $70,98 \%$ & $73,84 \%$ & $71,25 \%$ & $69,68 \%$ \\
\hline & & $\mathrm{CB}$ & $87,28 \%$ & 0,004 & $86,99 \%$ & $87,87 \%$ & $87,58 \%$ & $87,33 \%$ & $86,76 \%$ & $87,61 \%$ & $87,58 \%$ & $86,50 \%$ \\
\hline & & SP & $89,20 \%$ & 0,003 & $88,89 \%$ & $89,70 \%$ & $89,35 \%$ & $88,99 \%$ & $89,04 \%$ & $89,52 \%$ & $89,41 \%$ & $88,66 \%$ \\
\hline & & $\mathrm{LZ}$ & $89,57 \%$ & 0,003 & $89,36 \%$ & $90,02 \%$ & $89,75 \%$ & $89,30 \%$ & $89,28 \%$ & $89,92 \%$ & $89,73 \%$ & $89,22 \%$ \\
\hline \multirow{6}{*}{ 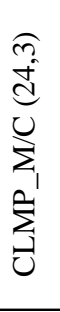 } & \multirow{6}{*}{ ก } & HW & $78,88 \%$ & 0,015 & $77,00 \%$ & $75,66 \%$ & $79,76 \%$ & $79,43 \%$ & $79,81 \%$ & $79,89 \%$ & $79,13 \%$ & $80,39 \%$ \\
\hline & & NN & $79,87 \%$ & 0,003 & $79,73 \%$ & $79,76 \%$ & $79,84 \%$ & $79,84 \%$ & $79,97 \%$ & $80,28 \%$ & $80,20 \%$ & $79,36 \%$ \\
\hline & & $\mathrm{LN}$ & $76,78 \%$ & 0,006 & $76,34 \%$ & $77,16 \%$ & $76,88 \%$ & $76,58 \%$ & $76,29 \%$ & $77,53 \%$ & $77,71 \%$ & $75,72 \%$ \\
\hline & & $\mathrm{CB}$ & $79,87 \%$ & 0,003 & $79,73 \%$ & $79,76 \%$ & $79,84 \%$ & $79,84 \%$ & $79,97 \%$ & $80,28 \%$ & $80,20 \%$ & $79,36 \%$ \\
\hline & & SP & $80,11 \%$ & 0,004 & $79,97 \%$ & $80,02 \%$ & $79,47 \%$ & $80,21 \%$ & $80,36 \%$ & $80,61 \%$ & $80,60 \%$ & $79,60 \%$ \\
\hline & & $\mathrm{LZ}$ & $80,15 \%$ & 0,003 & $80,02 \%$ & $80,01 \%$ & $79,72 \%$ & $80,19 \%$ & $80,43 \%$ & $80,78 \%$ & $80,46 \%$ & $79,60 \%$ \\
\hline \multirow{6}{*}{ 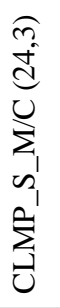 } & \multirow{6}{*}{$\stackrel{\infty}{\sim}$} & HW & $90,28 \%$ & 0,014 & $88,00 \%$ & $88,23 \%$ & $91,55 \%$ & $91,20 \%$ & $91,11 \%$ & $91,02 \%$ & $89,21 \%$ & $91,91 \%$ \\
\hline & & $\mathrm{NN}$ & $92,57 \%$ & 0,002 & $92,78 \%$ & $92,53 \%$ & $92,32 \%$ & $92,33 \%$ & $92,42 \%$ & $92,84 \%$ & $92,88 \%$ & $92,44 \%$ \\
\hline & & $\mathrm{LN}$ & $88,80 \%$ & 0,005 & $88,32 \%$ & $89,22 \%$ & $89,04 \%$ & $88,34 \%$ & $88,06 \%$ & $89,76 \%$ & $89,36 \%$ & $88,31 \%$ \\
\hline & & $\mathrm{CB}$ & $92,57 \%$ & 0,002 & $92,78 \%$ & $92,53 \%$ & $92,32 \%$ & $92,33 \%$ & $92,42 \%$ & $92,84 \%$ & $92,88 \%$ & $92,44 \%$ \\
\hline & & SP & $92,88 \%$ & 0,002 & $92,90 \%$ & $92,83 \%$ & $92,63 \%$ & $92,92 \%$ & $92,68 \%$ & $93,28 \%$ & $93,00 \%$ & $92,80 \%$ \\
\hline & & $\mathrm{LZ}$ & $92,84 \%$ & 0,001 & $92,87 \%$ & $92,83 \%$ & $92,76 \%$ & $92,66 \%$ & $92,59 \%$ & $93,02 \%$ & $93,07 \%$ & $92,90 \%$ \\
\hline \multirow{6}{*}{ 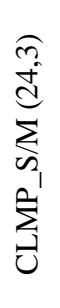 } & \multirow{6}{*}{$\frac{6}{6}$} & HW & $89,21 \%$ & 0,015 & $87,60 \%$ & $87,85 \%$ & $90,45 \%$ & $90,33 \%$ & $90,16 \%$ & $90,47 \%$ & $86,28 \%$ & $90,55 \%$ \\
\hline & & NN & $90,82 \%$ & 0,004 & $90,24 \%$ & $91,11 \%$ & $90,15 \%$ & $91,02 \%$ & $91,02 \%$ & $91,20 \%$ & $91,32 \%$ & $90,53 \%$ \\
\hline & & $\mathrm{LN}$ & $85,12 \%$ & 0,008 & $84,06 \%$ & $85,27 \%$ & $86,06 \%$ & $84,15 \%$ & $84,81 \%$ & $86,72 \%$ & $85,30 \%$ & $84,59 \%$ \\
\hline & & $\mathrm{CB}$ & $90,82 \%$ & 0,004 & $90,24 \%$ & $91,11 \%$ & $90,15 \%$ & $91,02 \%$ & $91,02 \%$ & $91,20 \%$ & $91,32 \%$ & $90,53 \%$ \\
\hline & & SP & $91,39 \%$ & 0,003 & $91,31 \%$ & $91,80 \%$ & $91,04 \%$ & $90,97 \%$ & $91,44 \%$ & $91,56 \%$ & $91,88 \%$ & $91,13 \%$ \\
\hline & & $\mathrm{LZ}$ & $91,33 \%$ & 0,002 & $91,32 \%$ & $91,46 \%$ & $91,13 \%$ & $90,99 \%$ & $91,67 \%$ & & & $91,19 \%$ \\
\hline \multirow{6}{*}{ 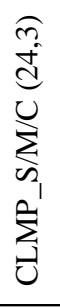 } & \multirow{6}{*}{ 尚 } & HW & $89,43 \%$ & 0,012 & $88,16 \%$ & $87,66 \%$ & $90,74 \%$ & $90,41 \%$ & $90,04 \%$ & $90,40 \%$ & $87,54 \%$ & $90,48 \%$ \\
\hline & & NN & $89,13 \%$ & 0,002 & $88,82 \%$ & $89,08 \%$ & $89,04 \%$ & & $89,36 \%$ & & $89,41 \%$ & $88,72 \%$ \\
\hline & & $\mathrm{LN}$ & $84,16 \%$ & 0,006 & $83,21 \%$ & $84,14 \%$ & $84,81 \%$ & $83,13 \%$ & $84,78 \%$ & $84,95 \%$ & $84,48 \%$ & $83,79 \%$ \\
\hline & & $\mathrm{CB}$ & $89,13 \%$ & 0,002 & $88,82 \%$ & $89,08 \%$ & $89,04 \%$ & $89,21 \%$ & $89,36 \%$ & $89,36 \%$ & $89,41 \%$ & $88,72 \%$ \\
\hline & & SP & $90,04 \%$ & 0,002 & $89,83 \%$ & $90,16 \%$ & $89,71 \%$ & $89,97 \%$ & $90,25 \%$ & $90,21 \%$ & $90,40 \%$ & $89,80 \%$ \\
\hline & & $\mathrm{LZ}$ & $90,36 \%$ & 0,003 & $90,22 \%$ & $90,54 \%$ & $89,97 \%$ & $90,48 \%$ & $90,61 \%$ & $90,72 \%$ & $90,43 \%$ & $89,93 \%$ \\
\hline \multirow{6}{*}{ 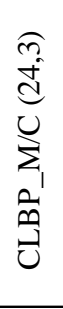 } & \multirow{6}{*}{ in } & HW & $76,87 \%$ & 0,012 & $75,70 \%$ & $74,10 \%$ & $77,65 \%$ & $77,73 \%$ & $77,82 \%$ & $77,38 \%$ & $76,76 \%$ & $77,79 \%$ \\
\hline & & NN & $77,88 \%$ & 0,002 & $77,74 \%$ & $77,88 \%$ & $77,59 \%$ & $78,08 \%$ & $77,81 \%$ & $78,20 \%$ & $78,15 \%$ & $77,55 \%$ \\
\hline & & $\mathrm{LN}$ & $71,24 \%$ & 0,007 & $70,40 \%$ & $71,11 \%$ & $72,42 \%$ & $71,34 \%$ & $70,68 \%$ & $72,51 \%$ & $71,23 \%$ & $70,26 \%$ \\
\hline & & $\mathrm{CB}$ & $77,88 \%$ & 0,002 & $77,74 \%$ & $77,88 \%$ & $77,59 \%$ & $78,08 \%$ & $77,81 \%$ & $78,20 \%$ & $78,15 \%$ & $77,55 \%$ \\
\hline & & SP & $79,64 \%$ & 0,002 & $79,49 \%$ & $79,76 \%$ & $79,54 \%$ & $80,00 \%$ & $79,39 \%$ & $79,80 \%$ & $79,77 \%$ & $79,33 \%$ \\
\hline & & $\mathrm{LZ}$ & $80,00 \%$ & 0,003 & $79,82 \%$ & $80,16 \%$ & $79,61 \%$ & $80,38 \%$ & $79,79 \%$ & $80,33 \%$ & $80,21 \%$ & $79,68 \%$ \\
\hline
\end{tabular}




\begin{tabular}{|c|c|c|c|c|c|c|c|c|c|c|c|c|}
\hline \multicolumn{13}{|c|}{$90 \times 90$ pixels } \\
\hline \multicolumn{3}{|c|}{ Dim. } & \multirow{2}{*}{$\begin{array}{c}\text { Média } \\
86,58 \%\end{array}$} & \multirow{2}{*}{$\begin{array}{c}\begin{array}{l}\text { Desvio } \\
\text { padrão }\end{array} \\
0,015\end{array}$} & \multirow{2}{*}{$\begin{array}{c}40^{\circ} \\
84,82 \%\end{array}$} & \multirow{2}{*}{$\begin{array}{c}80^{\circ} \\
84,64 \%\end{array}$} & \multirow{2}{*}{$\begin{array}{c}120^{\circ} \\
88,08 \%\end{array}$} & \multirow{2}{*}{$\begin{array}{c}160^{\circ} \\
87,69 \%\end{array}$} & \multirow{2}{*}{$\begin{array}{c}200^{\circ} \\
88,56 \%\end{array}$} & \multirow{2}{*}{$\begin{array}{c}240^{\circ} \\
87,99 \%\end{array}$} & \multirow{2}{*}{$\frac{280^{\circ}}{84,41 \%}$} & \multirow{2}{*}{$\frac{320^{\circ}}{86,45 \%}$} \\
\hline$\overparen{?}$ & & HW & & & & & & & & & & \\
\hline$\stackrel{d}{d}$ & \multirow{5}{*}{$\stackrel{\infty}{\sim}$} & $\mathrm{NN}$ & $86,54 \%$ & 0,006 & $85,36 \%$ & $87,24 \%$ & $86,19 \%$ & $86,63 \%$ & $86,24 \%$ & $86,89 \%$ & $87,57 \%$ & $86,19 \%$ \\
\hline 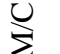 & & $\mathrm{LN}$ & $63,30 \%$ & 0,023 & $59,78 \%$ & $64,13 \%$ & $66,79 \%$ & $62,29 \%$ & $62,19 \%$ & $66,68 \%$ & $63,88 \%$ & $60,62 \%$ \\
\hline$\Omega_{1}$ & & $\mathrm{CB}$ & $86,54 \%$ & 0,006 & $85,36 \%$ & $87,24 \%$ & $86,19 \%$ & $86,63 \%$ & $86,24 \%$ & $86,89 \%$ & $87,57 \%$ & $86,19 \%$ \\
\hline n & & SP & $91,20 \%$ & 0,003 & $90,67 \%$ & $91,34 \%$ & $91,10 \%$ & $91,41 \%$ & $90,88 \%$ & $91,60 \%$ & $91,65 \%$ & $90,92 \%$ \\
\hline 己 & & $\mathrm{LZ}$ & $92,09 \%$ & 0,002 & $91,78 \%$ & $92,17 \%$ & $92,00 \%$ & $92,20 \%$ & $91,95 \%$ & $92,44 \%$ & $92,28 \%$ & $91,93 \%$ \\
\hline \multirow{6}{*}{ 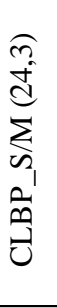 } & \multirow{6}{*}{$\frac{0}{6}$} & HW & $85,18 \%$ & 0,021 & $83,88 \%$ & $83,14 \%$ & $86,35 \%$ & $87,05 \%$ & $87,60 \%$ & $86,74 \%$ & $80,57 \%$ & $86,07 \%$ \\
\hline & & $\mathrm{NN}$ & $90,87 \%$ & 0,005 & $89,88 \%$ & $91,48 \%$ & $91,02 \%$ & $91,07 \%$ & $90,71 \%$ & $91,12 \%$ & $91,30 \%$ & $90,38 \%$ \\
\hline & & $\mathrm{LN}$ & $69,29 \%$ & 0,026 & $65,86 \%$ & $70,15 \%$ & $73,30 \%$ & $67,88 \%$ & $67,71 \%$ & $73,16 \%$ & $70,20 \%$ & $66,04 \%$ \\
\hline & & $\mathrm{CB}$ & $90,87 \%$ & 0,005 & $89,88 \%$ & $91,48 \%$ & $91,02 \%$ & $91,07 \%$ & $90,71 \%$ & $91,12 \%$ & $91,30 \%$ & $90,38 \%$ \\
\hline & & SP & $93,59 \%$ & 0,002 & $93,19 \%$ & $93,83 \%$ & $93,72 \%$ & $93,59 \%$ & $93,51 \%$ & $93,87 \%$ & $93,61 \%$ & $93,43 \%$ \\
\hline & & $\mathrm{LZ}$ & $93,95 \%$ & 0,002 & $93,77 \%$ & $93,85 \%$ & $94,10 \%$ & $94,03 \%$ & $93,81 \%$ & $94,28 \%$ & $94,08 \%$ & $93,70 \%$ \\
\hline \multirow{6}{*}{ 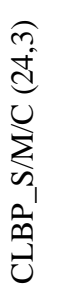 } & \multirow{6}{*}{$\stackrel{\sim}{n}$} & HW & $88,66 \%$ & 0,016 & $87,08 \%$ & $87,08 \%$ & $90,09 \%$ & $90,28 \%$ & $90,00 \%$ & $90,02 \%$ & $85,42 \%$ & $89,27 \%$ \\
\hline & & NN & $92,38 \%$ & 0,003 & $91,84 \%$ & $92,60 \%$ & $92,43 \%$ & $92,45 \%$ & $92,10 \%$ & $92,39 \%$ & $93,09 \%$ & $92,16 \%$ \\
\hline & & $\mathrm{LN}$ & $81,19 \%$ & 0,016 & $78,35 \%$ & $82,10 \%$ & $83,34 \%$ & $80,29 \%$ & $80,00 \%$ & $83,35 \%$ & $82,15 \%$ & $79,90 \%$ \\
\hline & & $\mathrm{CB}$ & $92,38 \%$ & 0,003 & $91,84 \%$ & $92,60 \%$ & $92,43 \%$ & $92,45 \%$ & $92,10 \%$ & $92,39 \%$ & $93,09 \%$ & $92,16 \%$ \\
\hline & & SP & $93,80 \%$ & 0,002 & $93,54 \%$ & $93,88 \%$ & $93,80 \%$ & $94,01 \%$ & $93,65 \%$ & $93,89 \%$ & $94,06 \%$ & $93,54 \%$ \\
\hline & & $\mathrm{LZ}$ & $93,89 \%$ & 0,002 & $93,82 \%$ & $93,80 \%$ & $93,94 \%$ & $94,12 \%$ & $93,68 \%$ & $94,17 \%$ & $93,95 \%$ & $93,65 \%$ \\
\hline
\end{tabular}




\section{APÊNDICE D - Acurácias médias obtidas pelos descritores avaliados no Experimento 4}

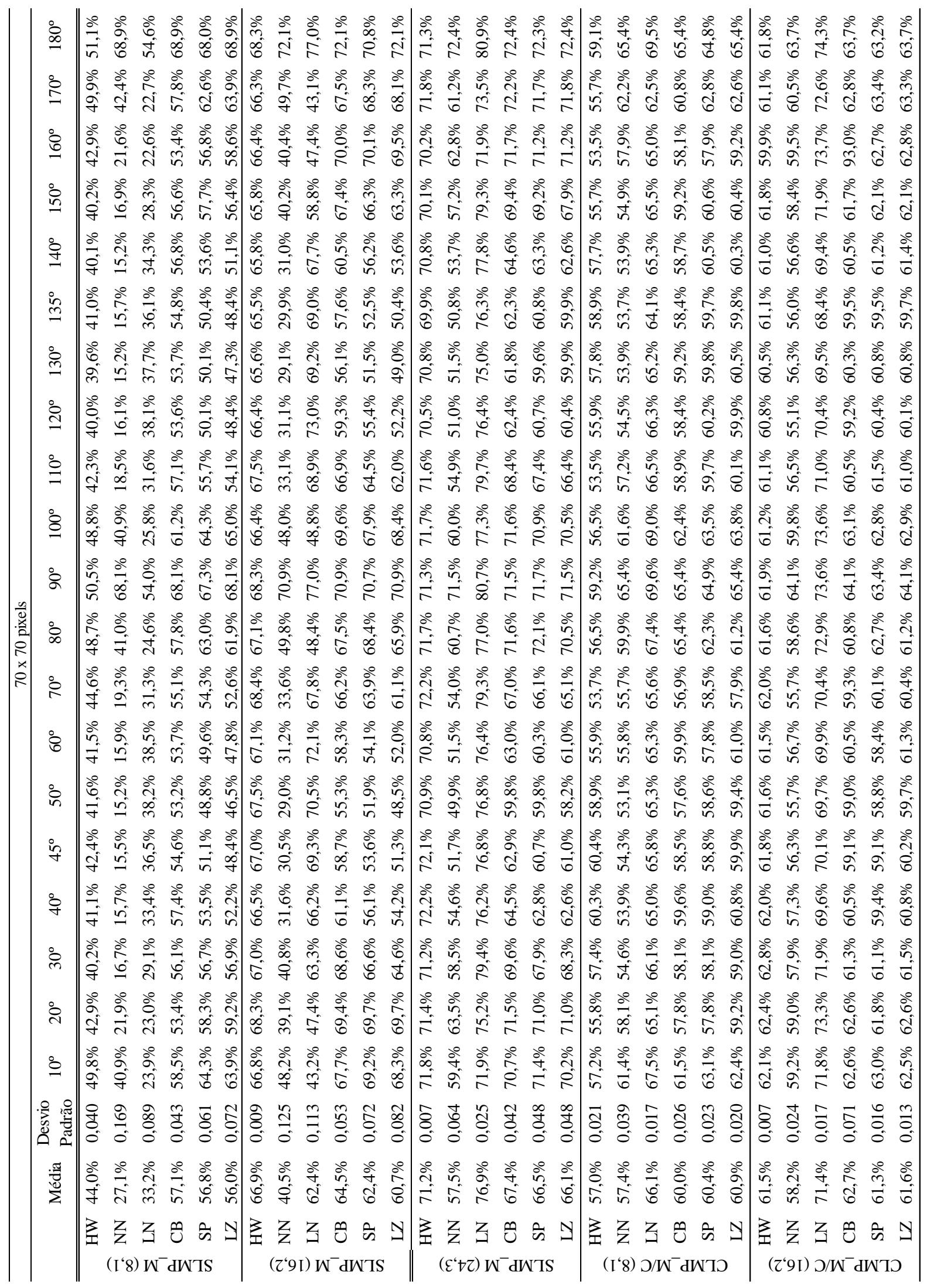




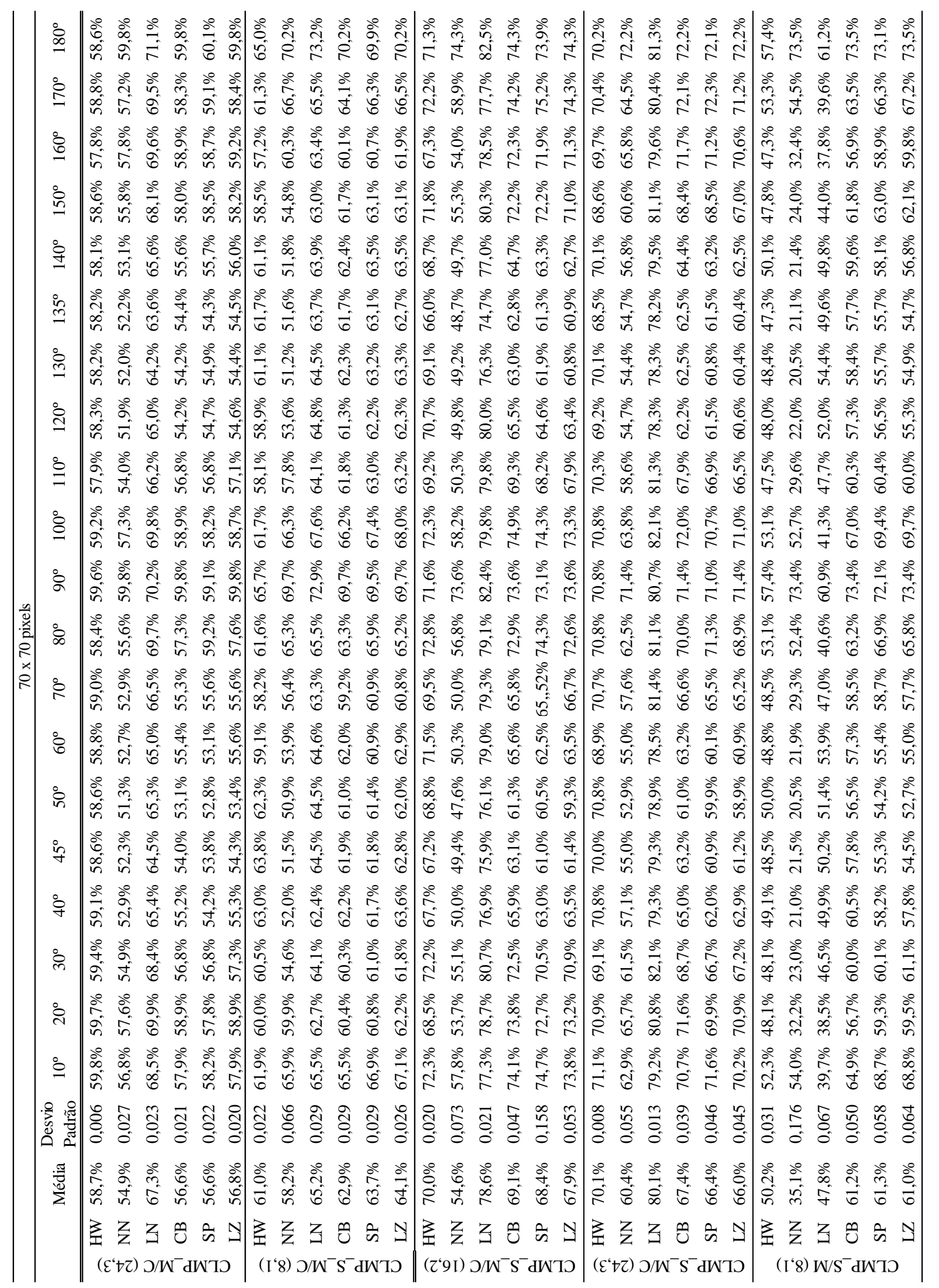




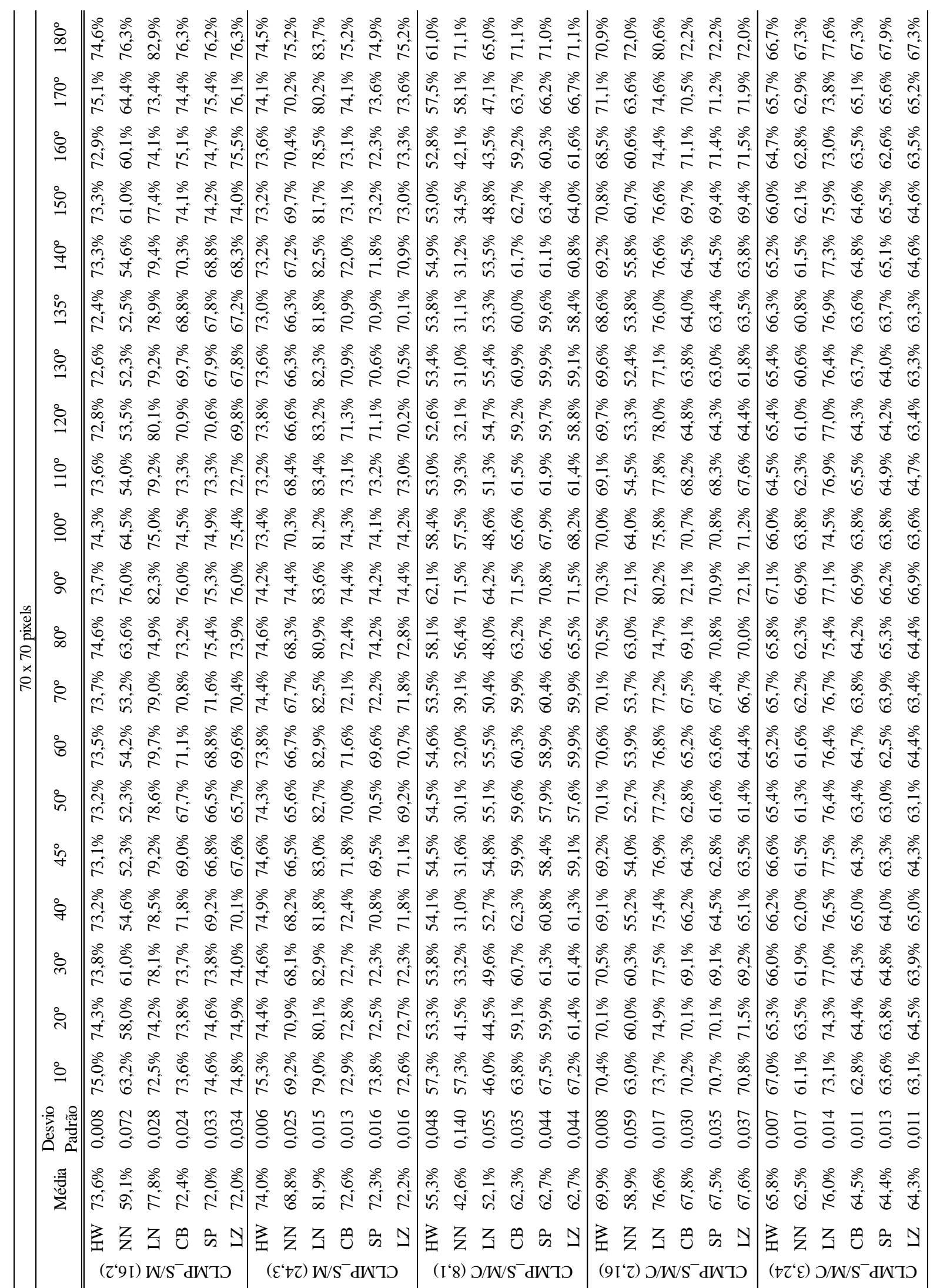




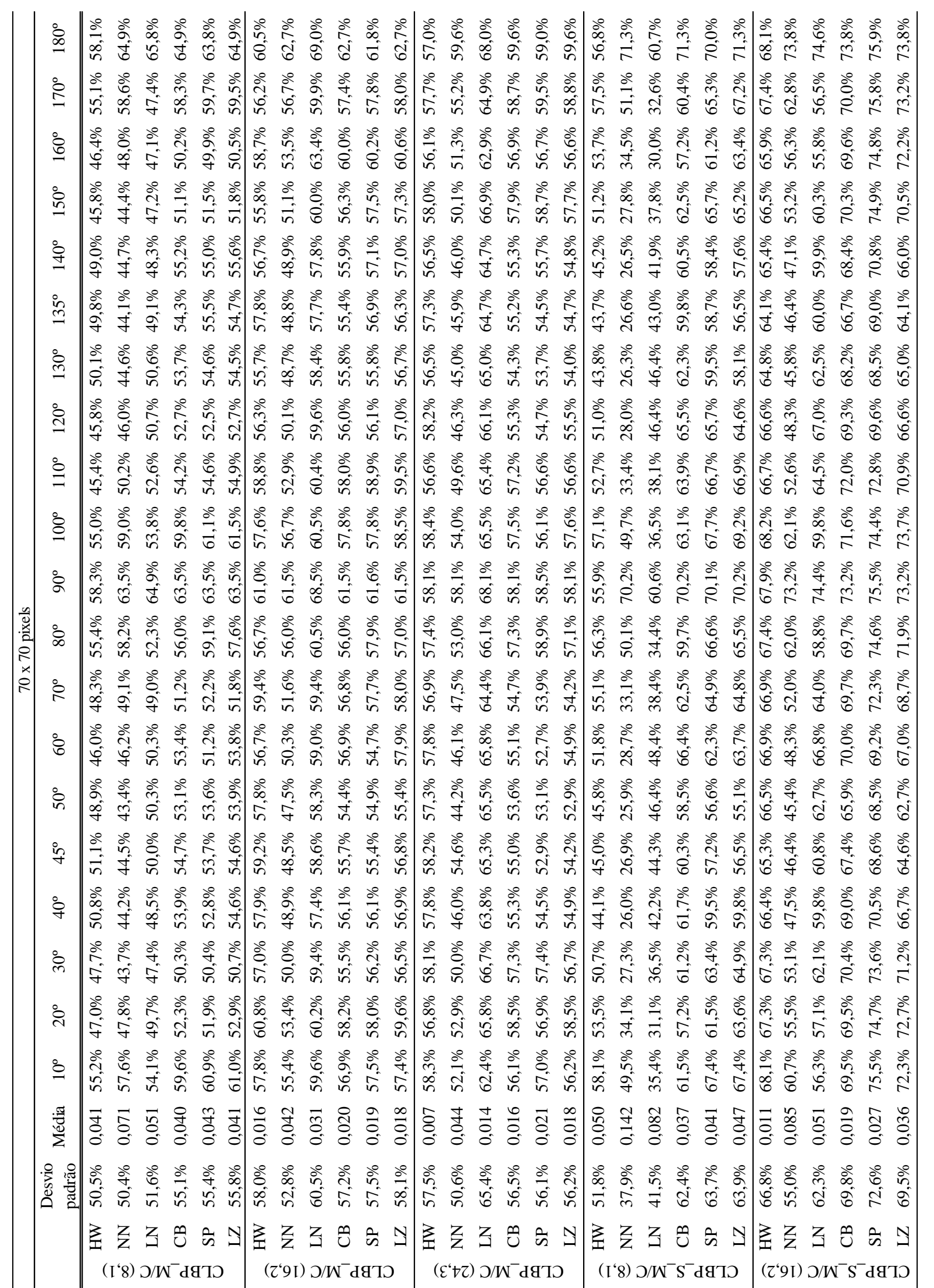




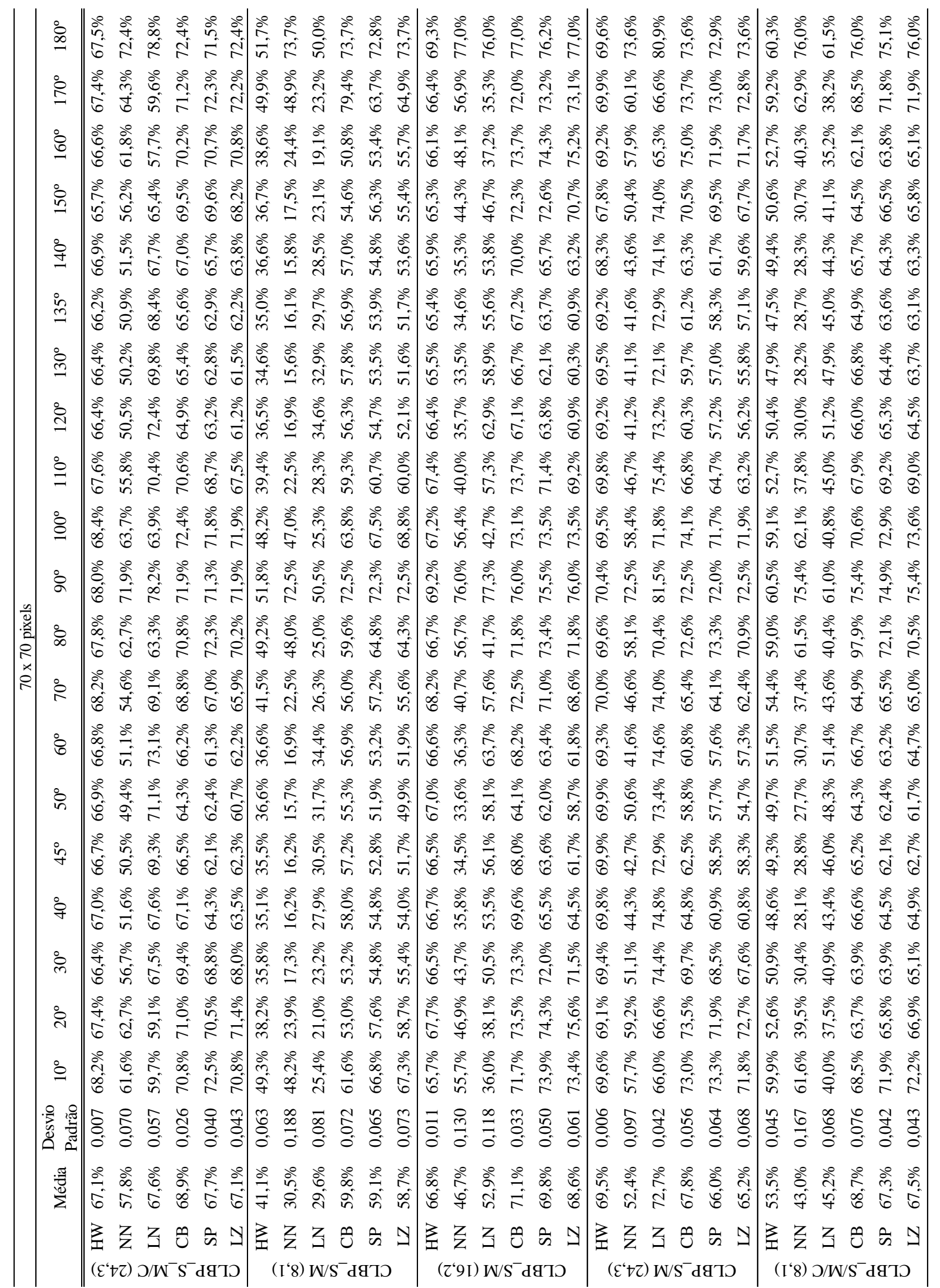




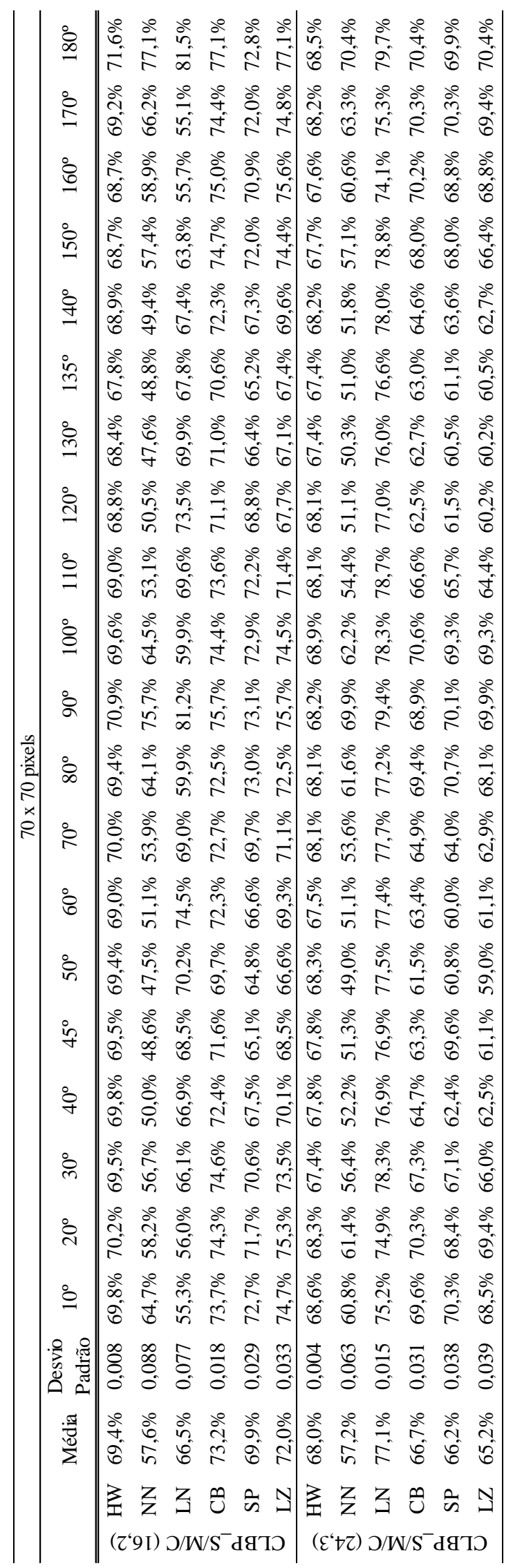




\begin{tabular}{|c|c|c|c|c|c|}
\hline & & & & & \\
\hline & & & & & \\
\hline$\stackrel{1}{i}$ & 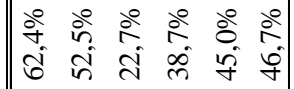 & 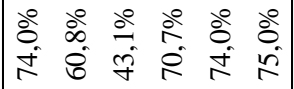 & 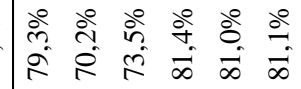 & 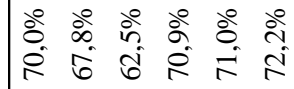 & 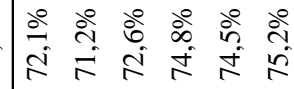 \\
\hline & 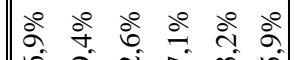 & & கீ & $\therefore$ \&ீ & 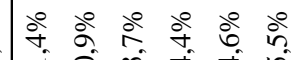 \\
\hline & के ते & 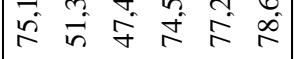 & 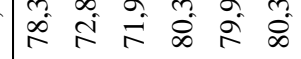 & $\begin{array}{lll}\infty & 8 & 0\end{array}$ & 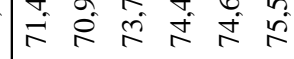 \\
\hline & 웅 & 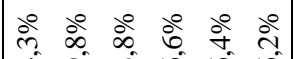 & 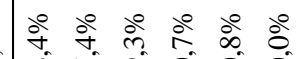 & $\because \because$ i̊ & 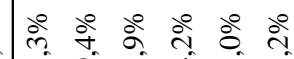 \\
\hline & 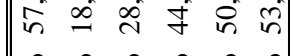 & 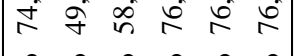 & $\therefore 5$ & 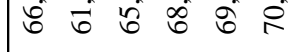 & 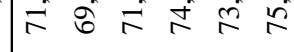 \\
\hline & 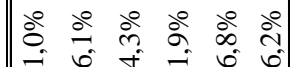 & 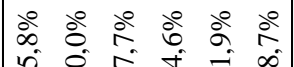 & 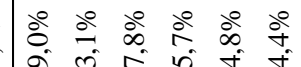 & $\begin{array}{c}0 \\
\therefore\end{array}$ & 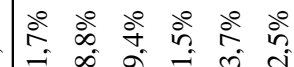 \\
\hline & $\left|\begin{array}{llllll}6 & - & c & \bar{n} & n & n \\
0 & 0 & 0 & 0 & 0 & 0\end{array}\right|$ & 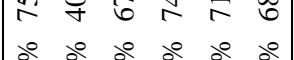 & 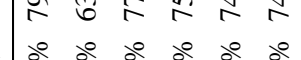 & 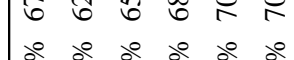 & $=00$ \\
\hline$\stackrel{i}{m}$ & 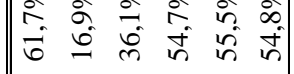 & 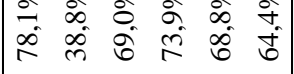 & 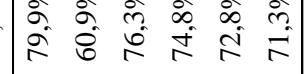 & 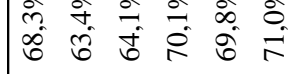 & 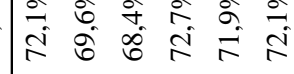 \\
\hline & 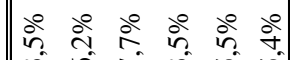 & 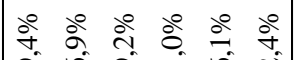 & 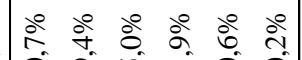 & $\therefore$ ¿ீ & 웜 \\
\hline & $80 \bar{n}$ in in & 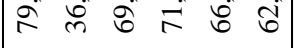 & $\infty$ in & $86882 ?$ & 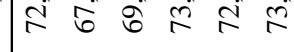 \\
\hline ¿ & 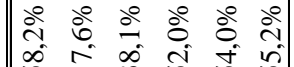 & 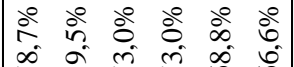 & 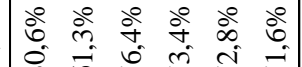 & ڤ̊ & 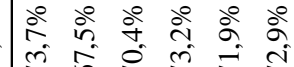 \\
\hline & 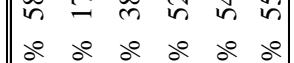 & 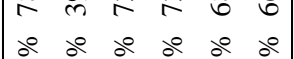 & 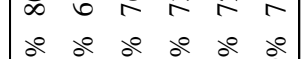 & $\begin{array}{llllll}0 & 0 & 0 & 0 & 0 & 0 \\
0 & 0 & 0 & 0 & 0 & 0\end{array}$ & $\therefore 505050$ \\
\hline & 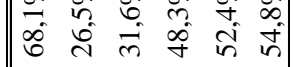 & 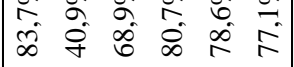 & 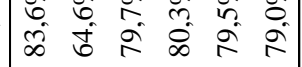 & 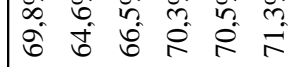 & 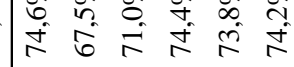 \\
\hline & 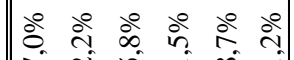 & $\therefore 0$ & $\ddot{~}$ & 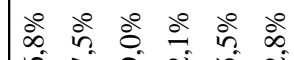 & 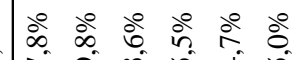 \\
\hline & 5 की & $\infty$ in $\infty$ क & 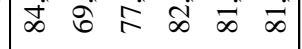 & $\therefore 88 \div 8$ & $\approx 8 \approx n$ \\
\hline & 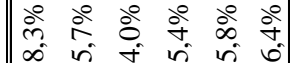 & 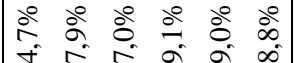 & 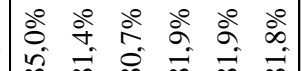 & 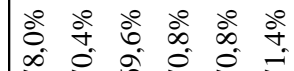 & $\begin{array}{lllll}0 & 0 & 0 & 0 & 0 \\
i & 0 & 0 \\
n & n & m & n & i\end{array}$ \\
\hline & 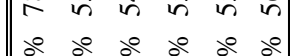 & $\mid \begin{array}{lll}\infty & i & i \\
0 & 0 & 0\end{array}$ & $\begin{array}{lllll}\infty & \infty & \infty & \infty & \infty \\
0 & 0 & \circ & \circ & \circ\end{array} \mid$ & $\begin{array}{lll}0 & 0 \\
0 & 0 & 0\end{array}$ & 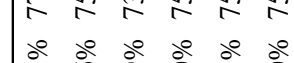 \\
\hline & 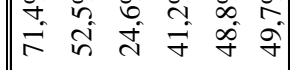 & 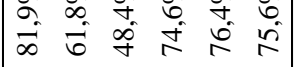 & 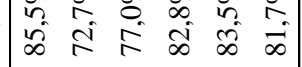 & 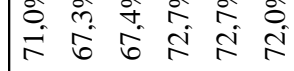 & 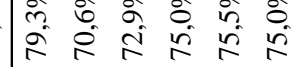 \\
\hline 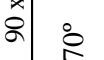 & 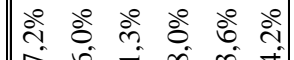 & 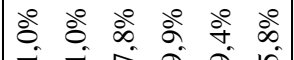 & 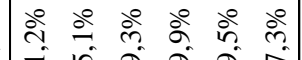 & $\therefore$ & 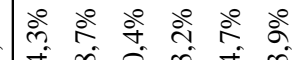 \\
\hline & in $\stackrel{i}{\bar{m}} \stackrel{\infty}{+} n$ & & 62 & $8: 3$ & to \\
\hline : & 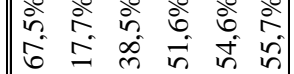 & 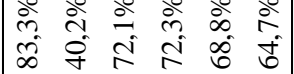 & 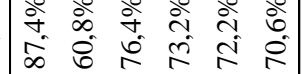 & 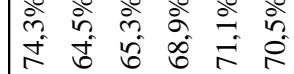 & 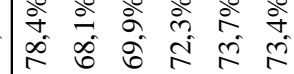 \\
\hline in & 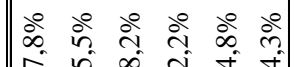 & 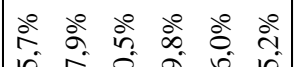 & 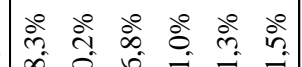 & 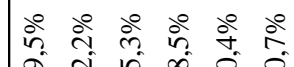 & 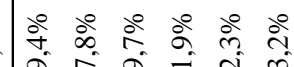 \\
\hline & 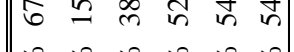 & 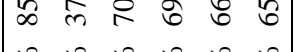 & $\infty \quad \infty \therefore ⿱ 一 ⿻ 上 丨$ & 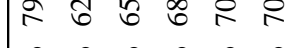 & $\therefore 285 \pi$ \\
\hline$i$ & 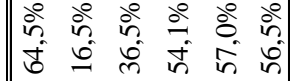 & 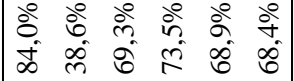 & 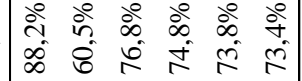 & 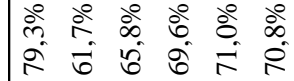 & 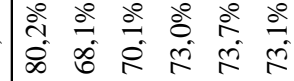 \\
\hline q & 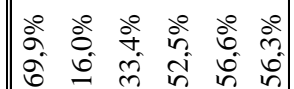 & 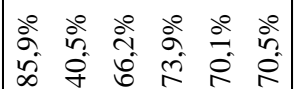 & 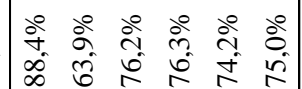 & 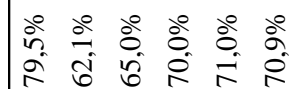 & 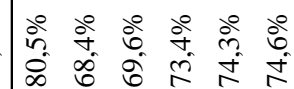 \\
\hline$\overbrace{}^{2}$ & $\therefore \therefore 0000$ & 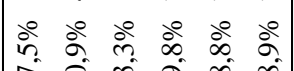 & 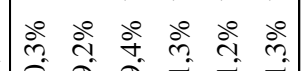 & $\stackrel{\circ}{m}$ mீ & $\stackrel{\circ}{\circ} \stackrel{\circ}{\circ}$ \\
\hline & 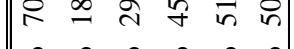 & $\infty$ in $\beta \approx 2 \infty$ & 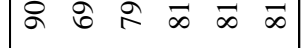 & $1=8888$ & $\bar{\infty} 5 \pi$ \\
\hline$\check{c}^{\circ}$ & 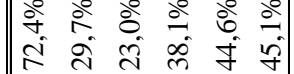 & 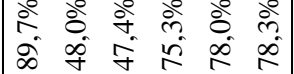 & 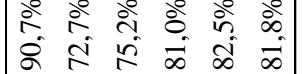 & 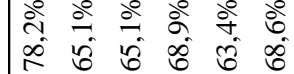 & 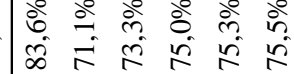 \\
\hline & 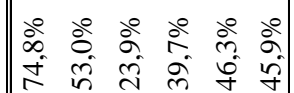 & 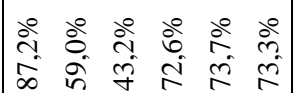 & 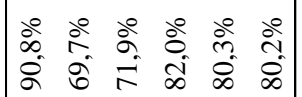 & 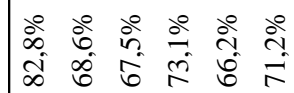 & 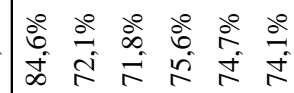 \\
\hline 墕 & 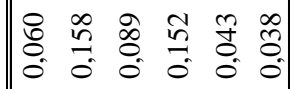 & 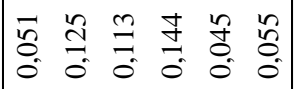 & 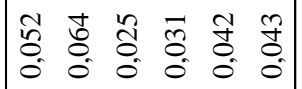 & 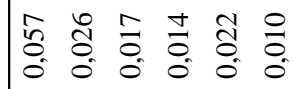 & 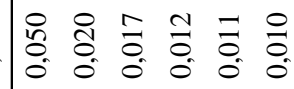 \\
\hline . & 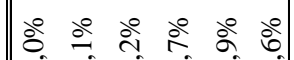 & $\therefore$ b̊ & 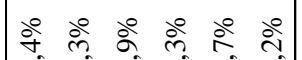 & இ & ڤ̊ \\
\hline & 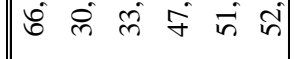 & 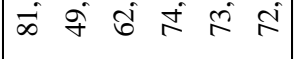 & $\infty 6 \div \approx$ & 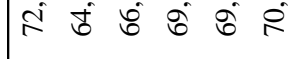 & $\therefore B \approx m$ \\
\hline & 茥 Z & 艺 Z & 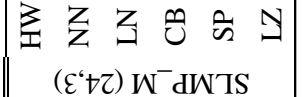 & 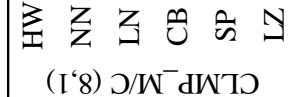 & Z \\
\hline
\end{tabular}




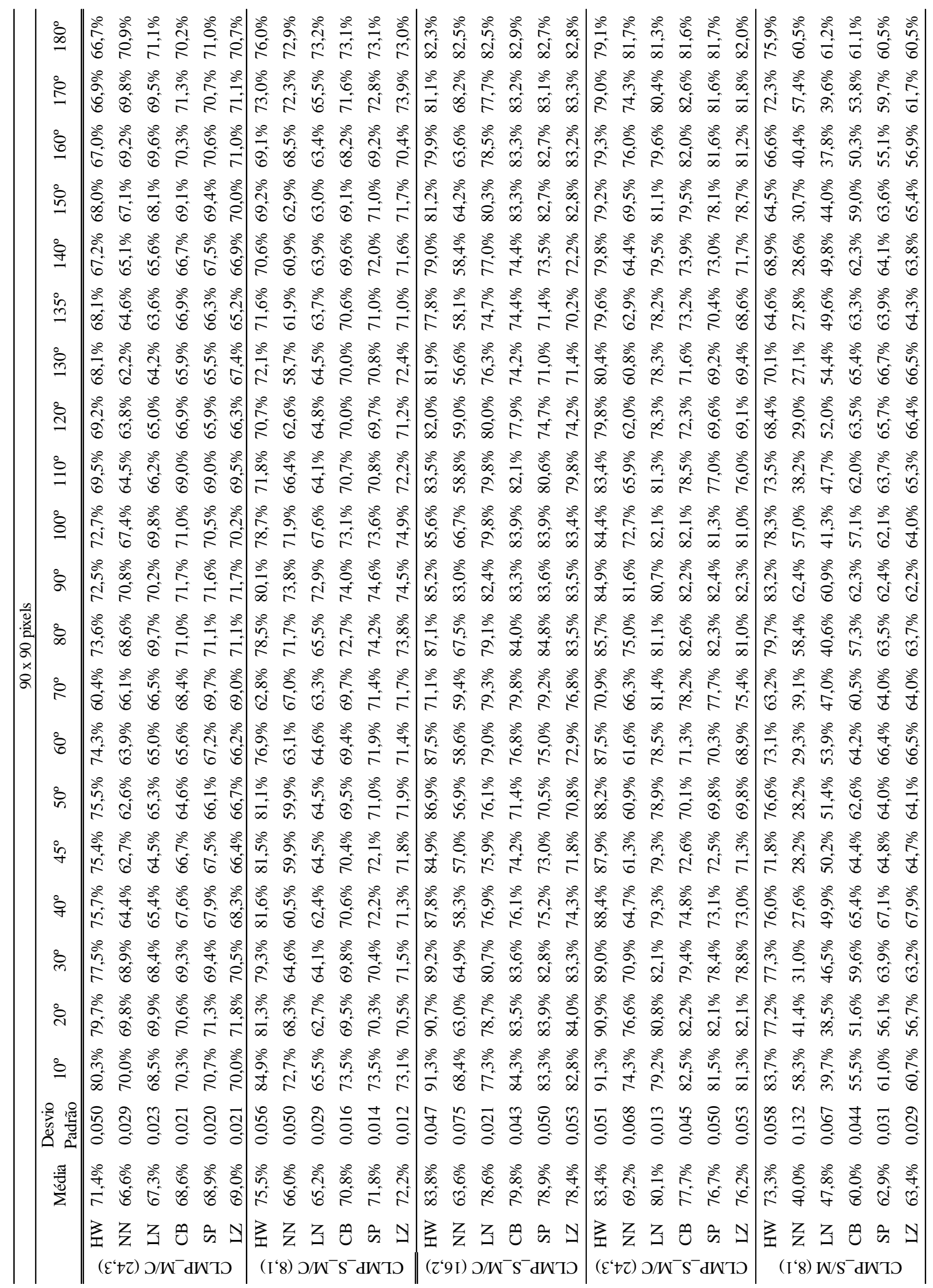




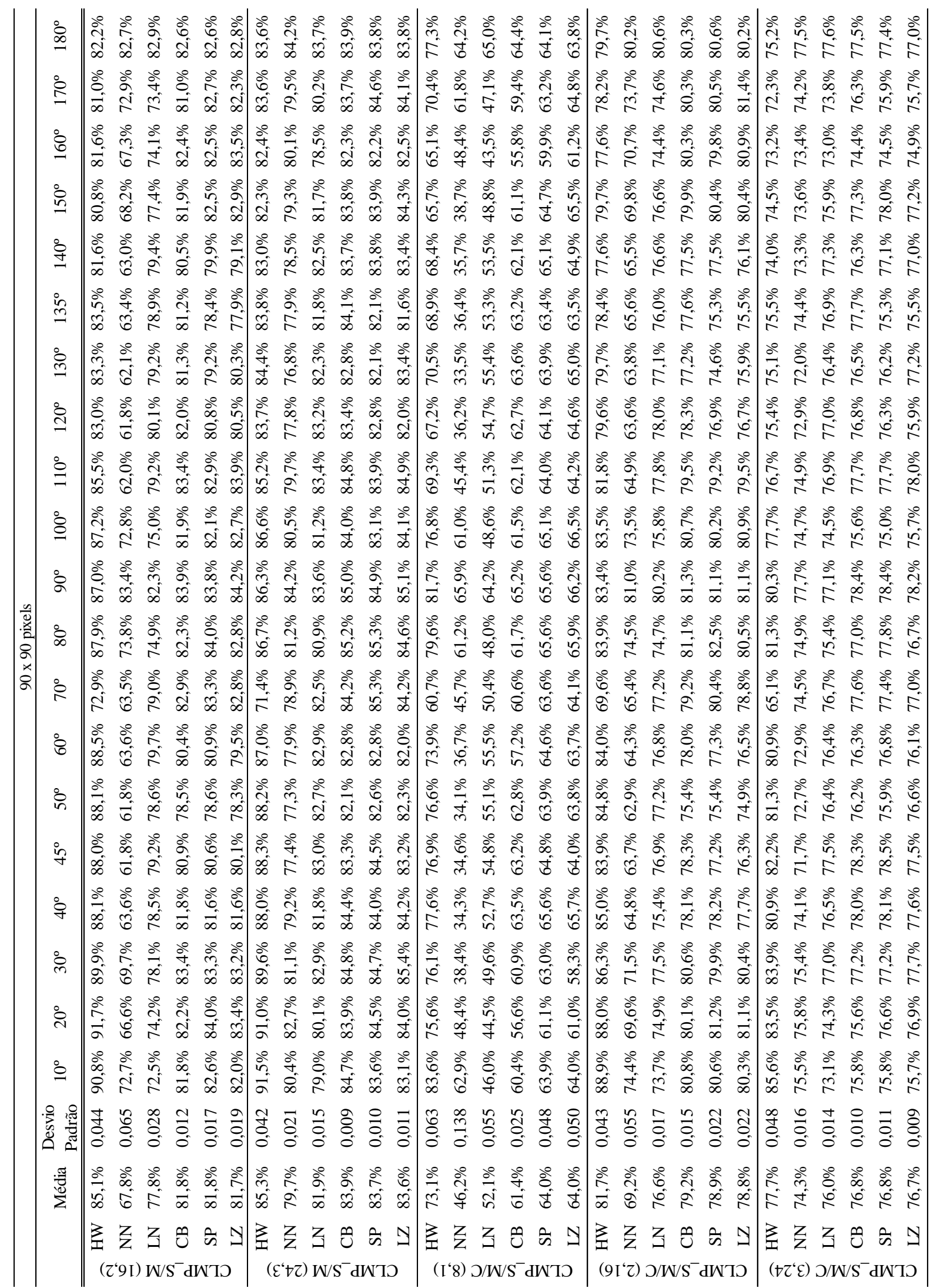




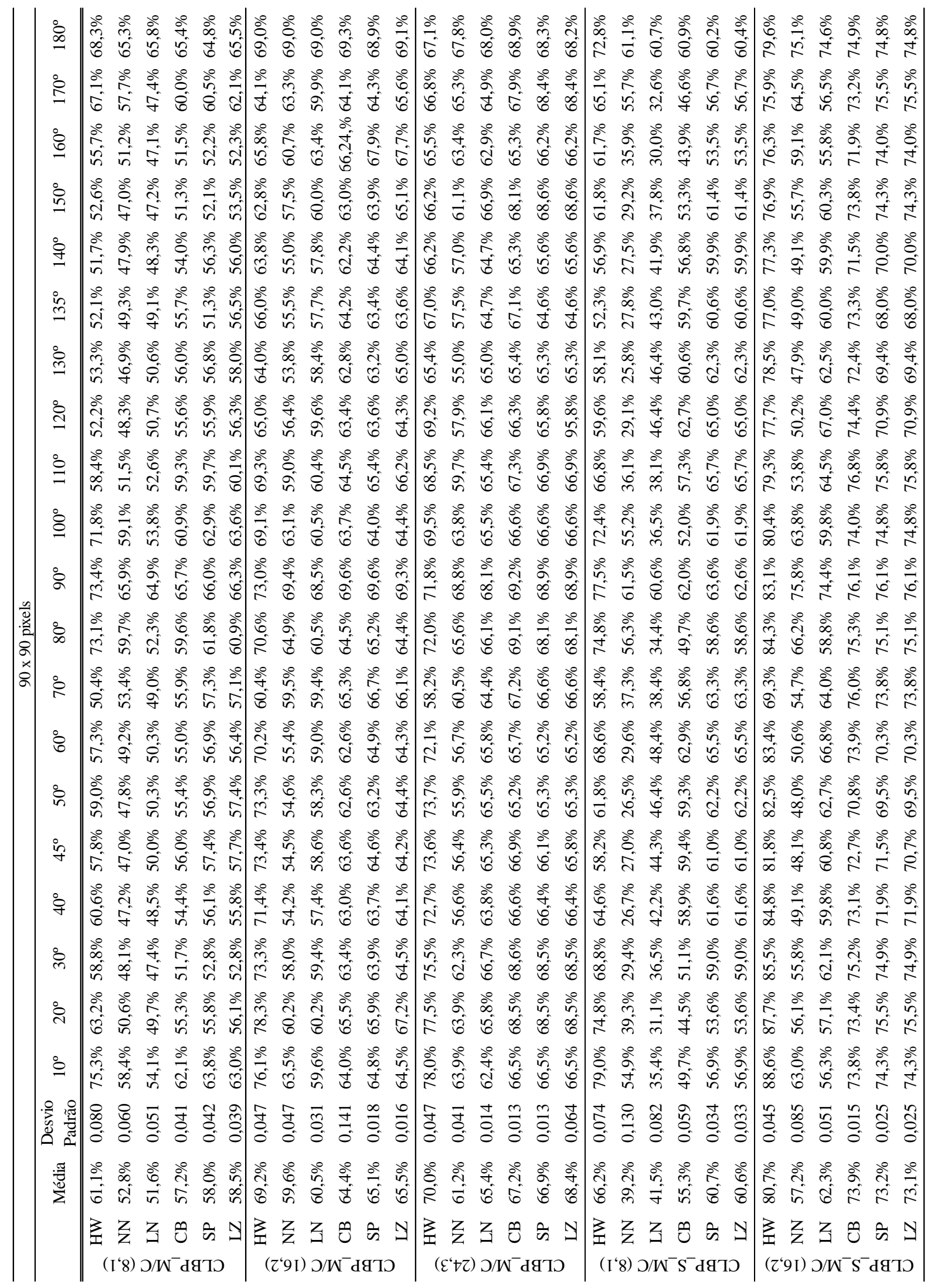




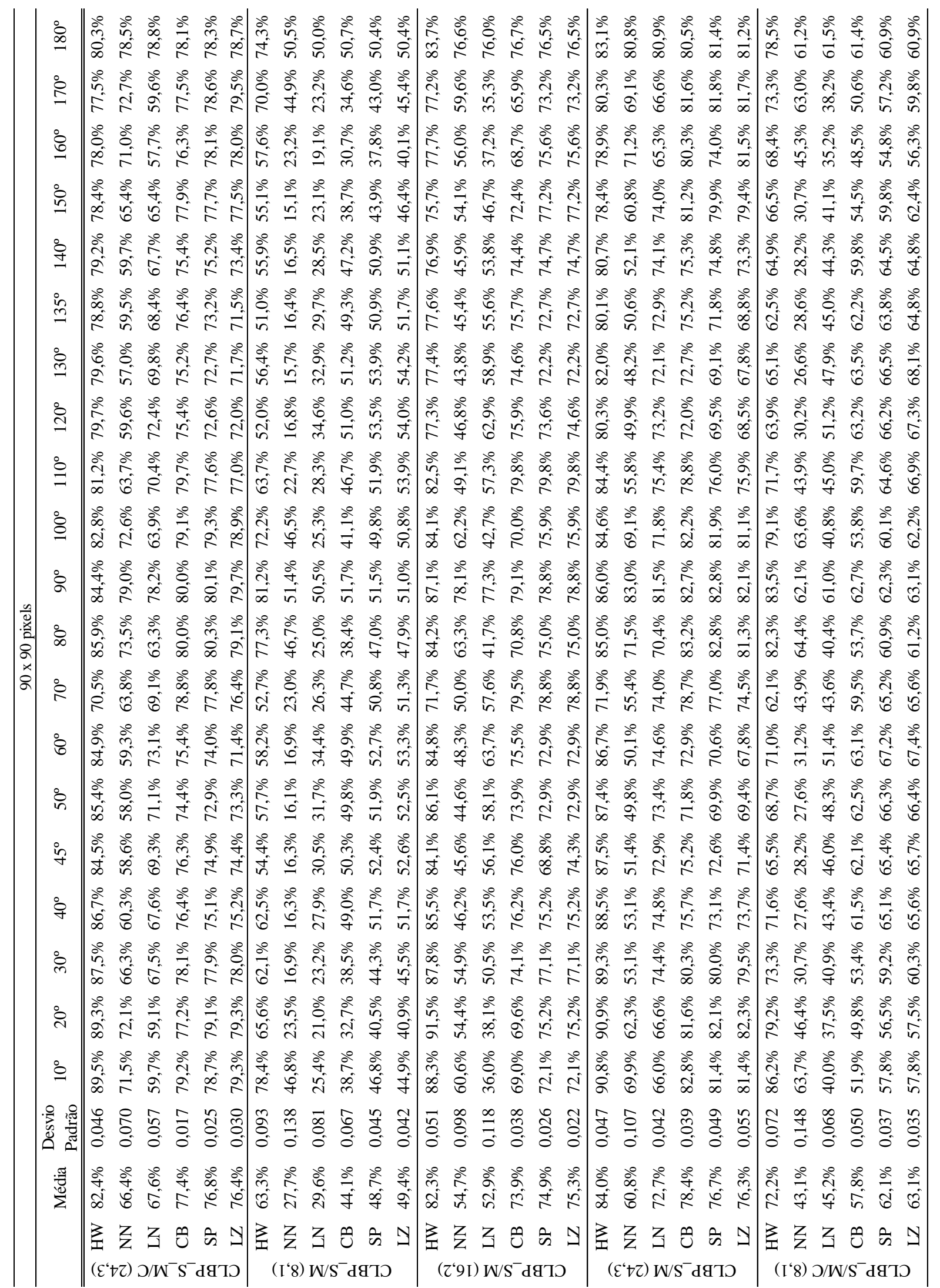




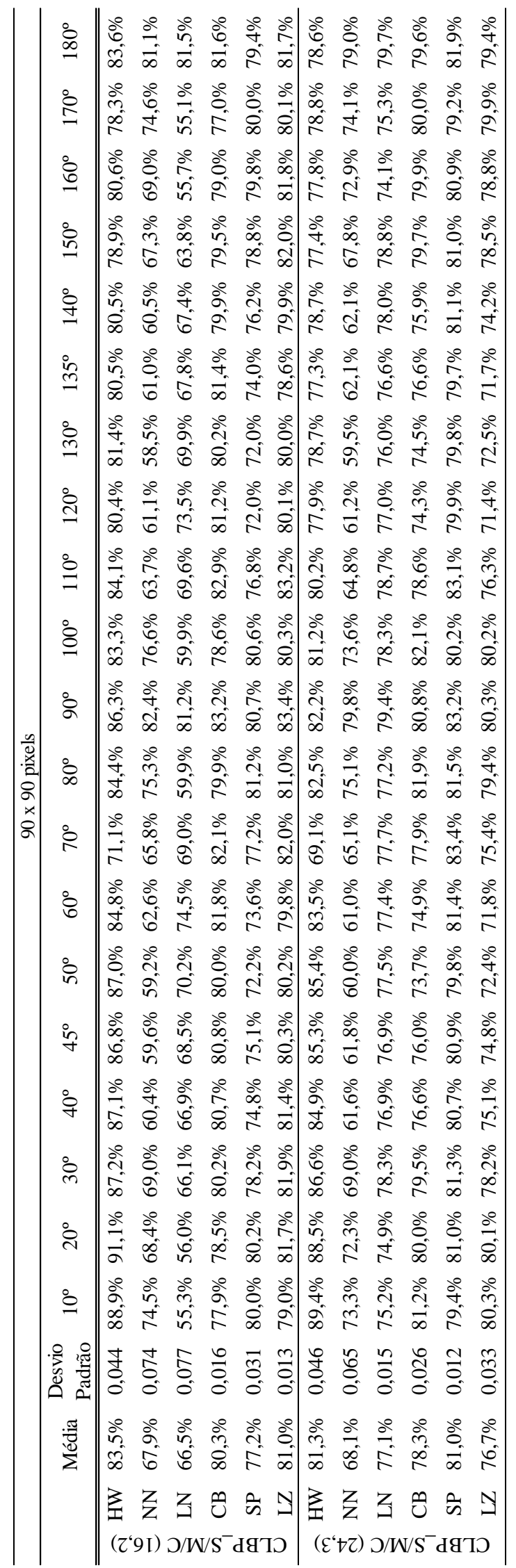




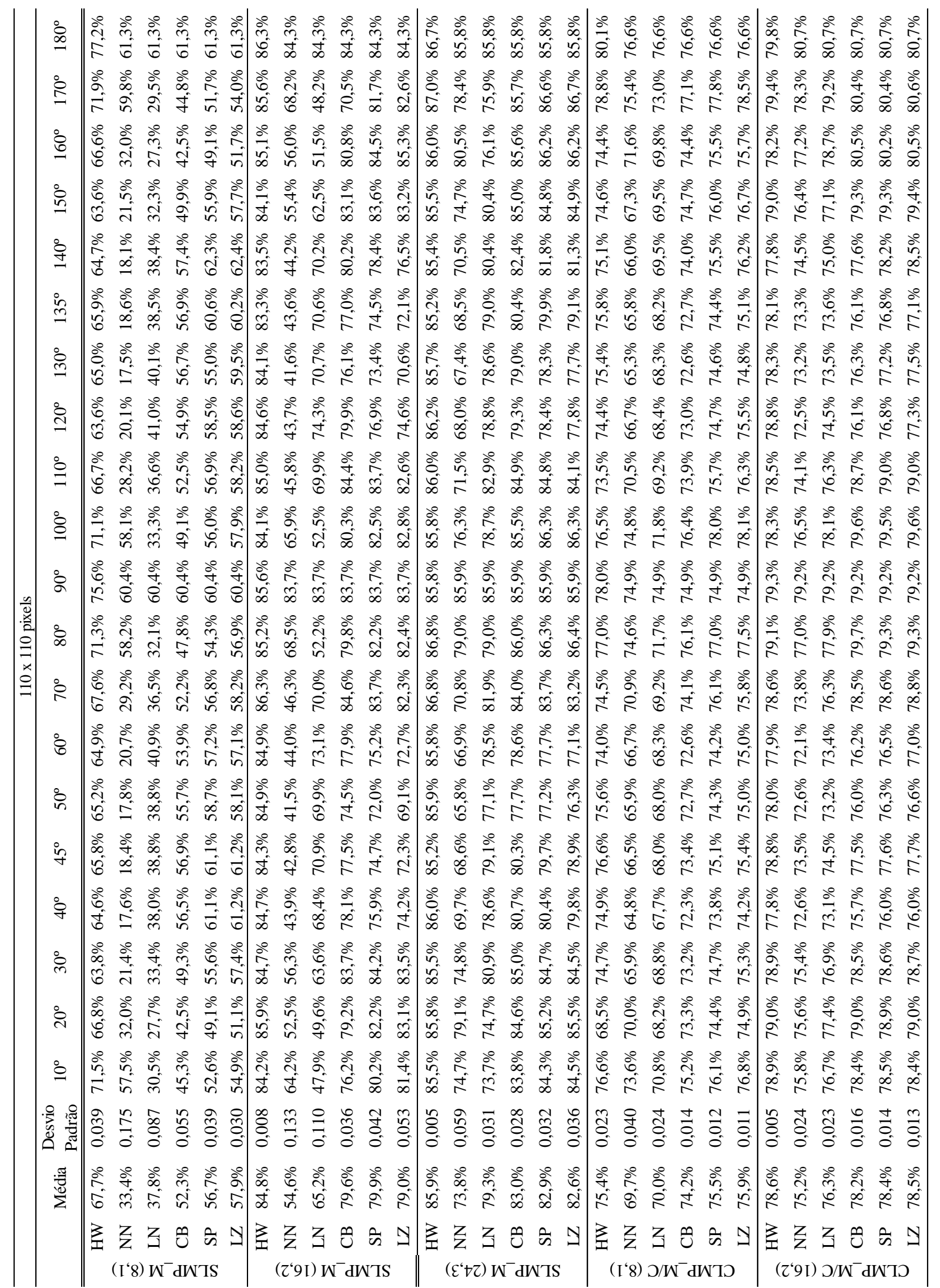




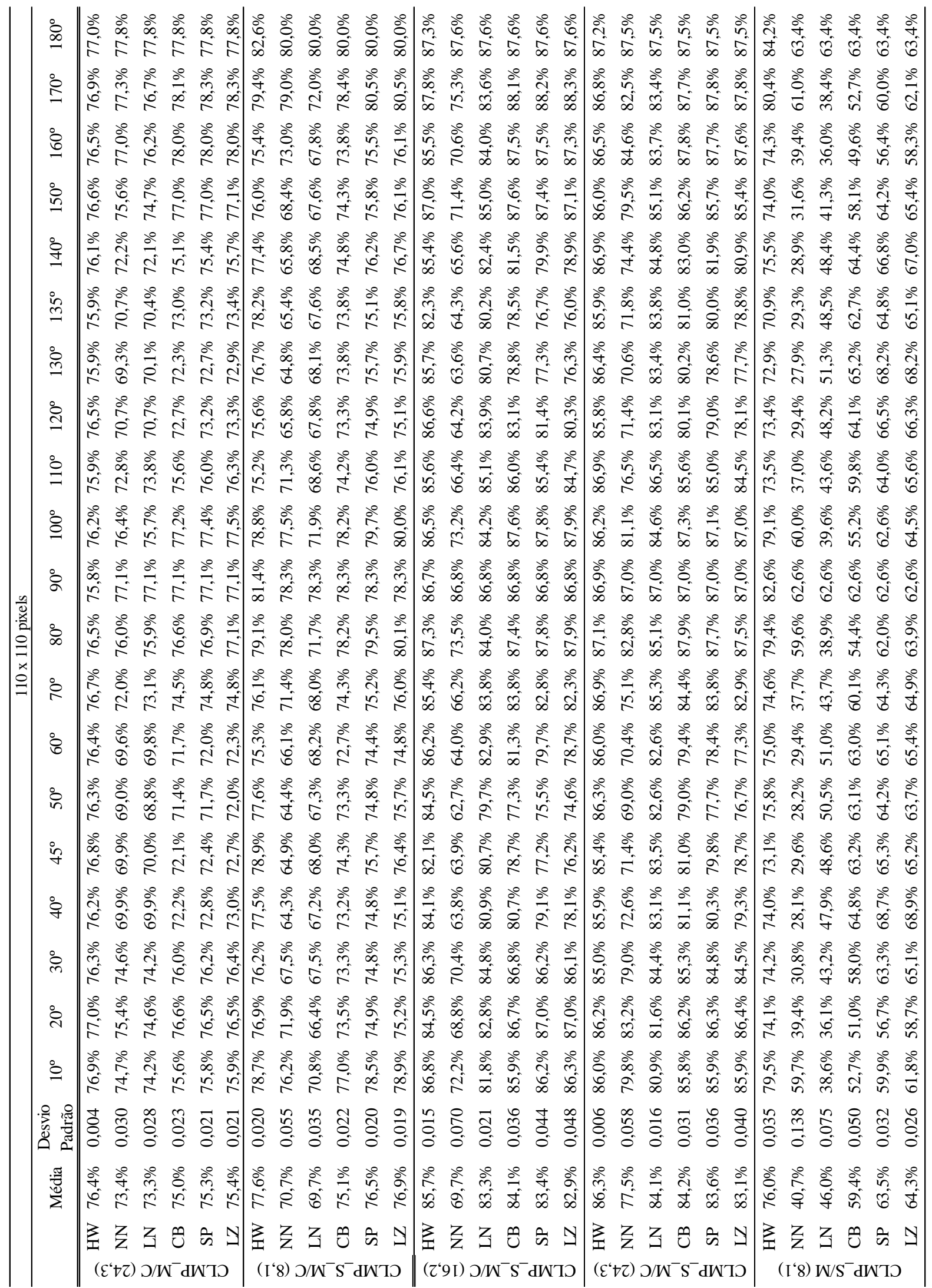




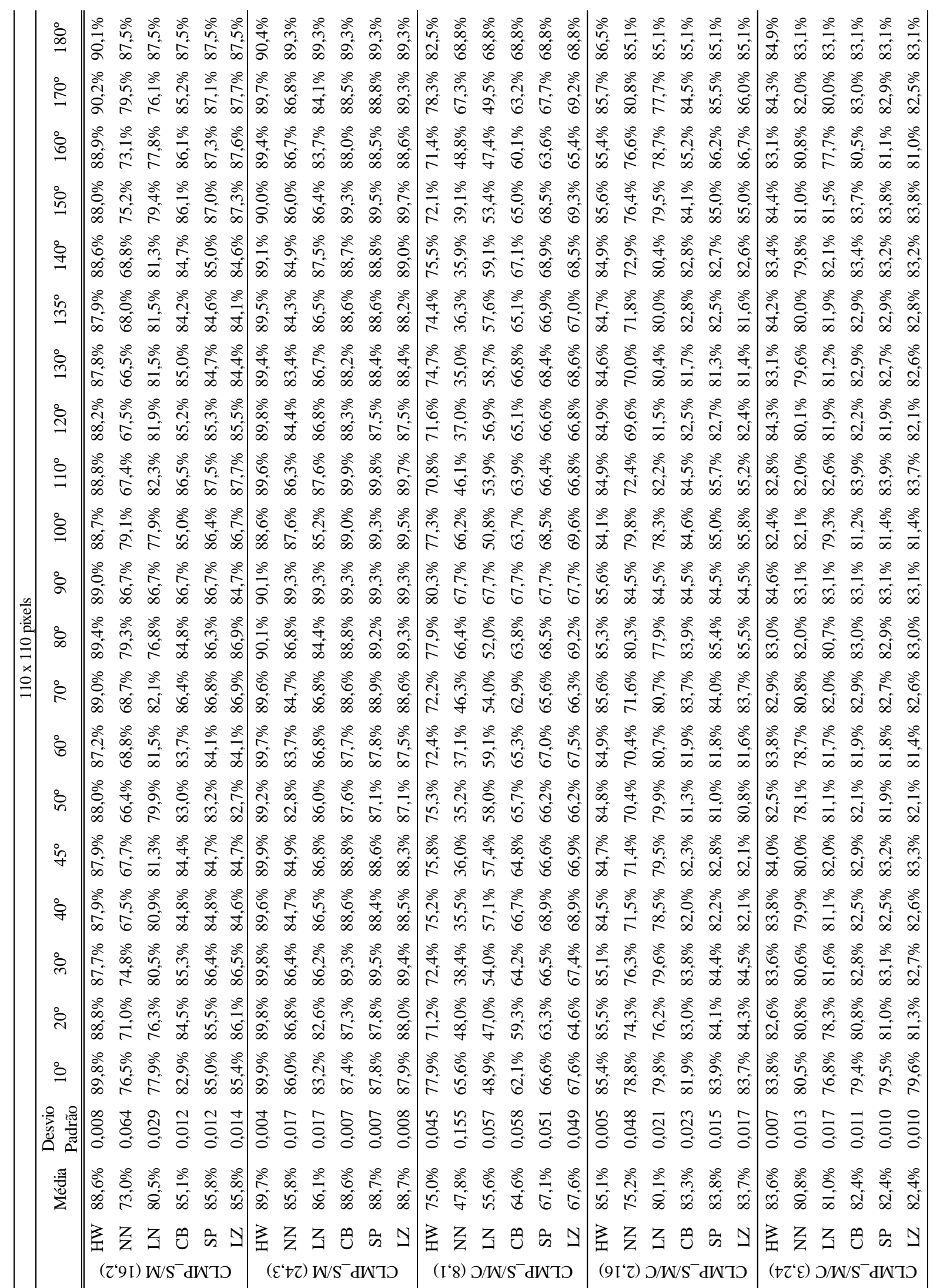




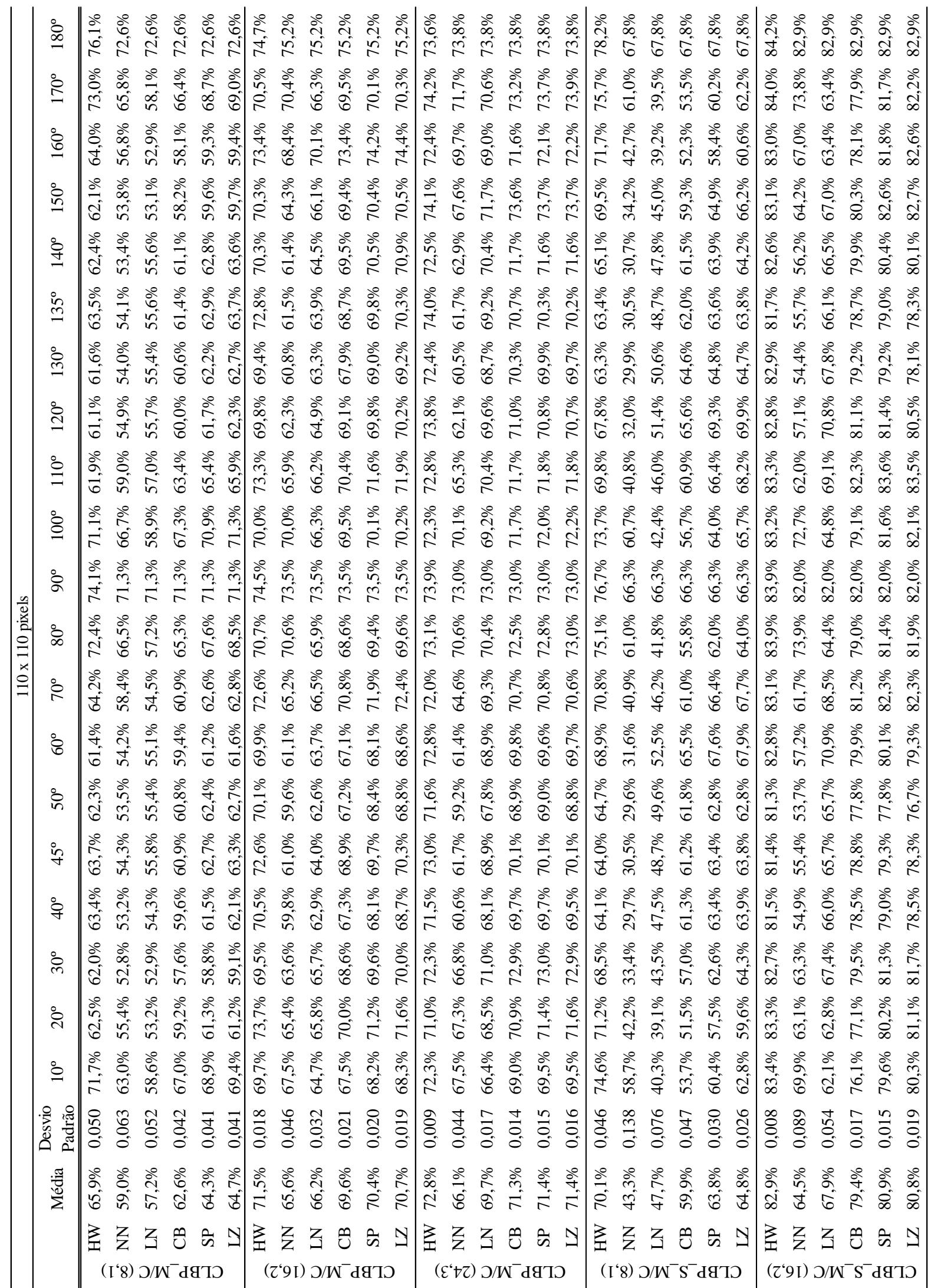




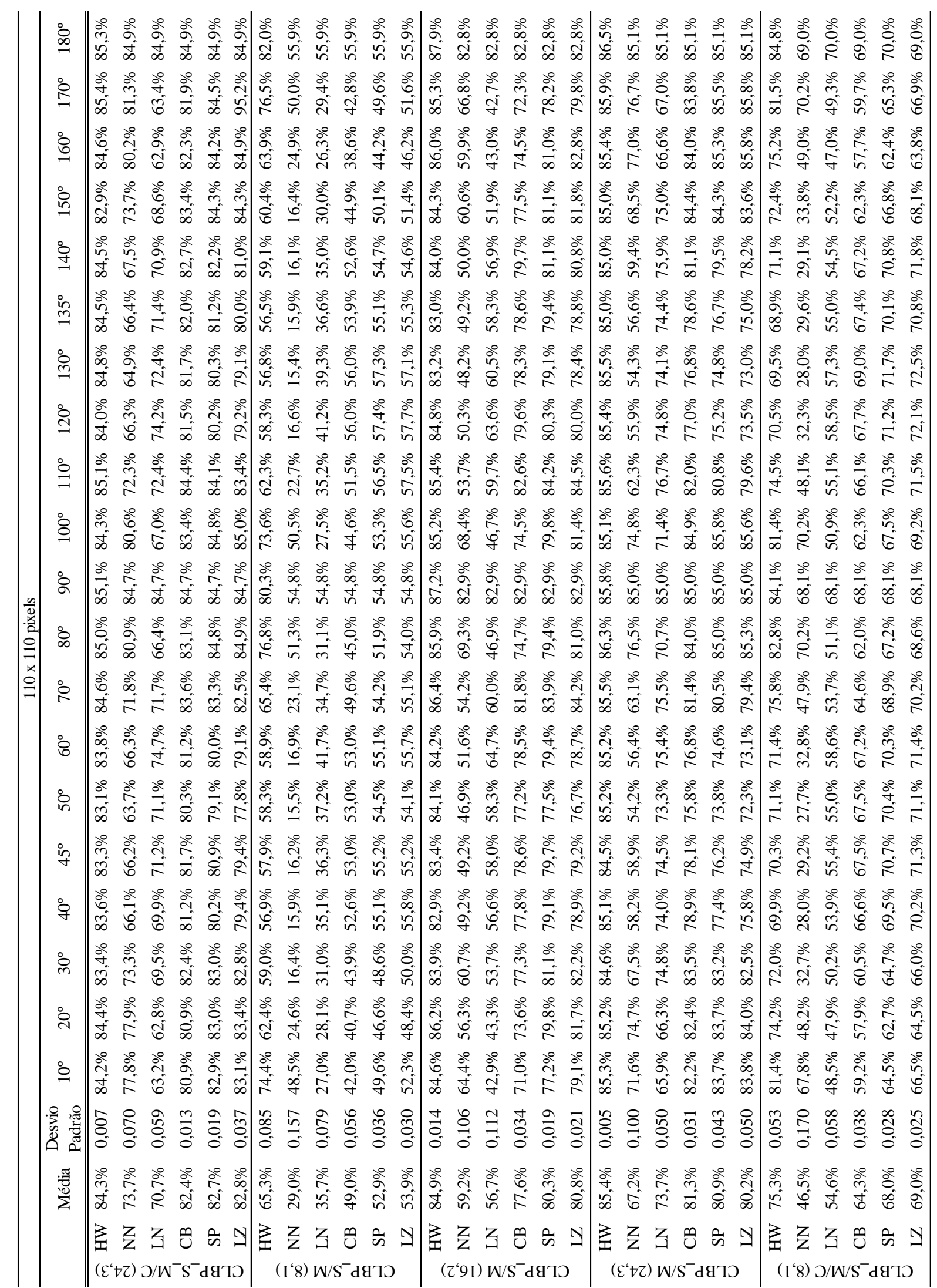




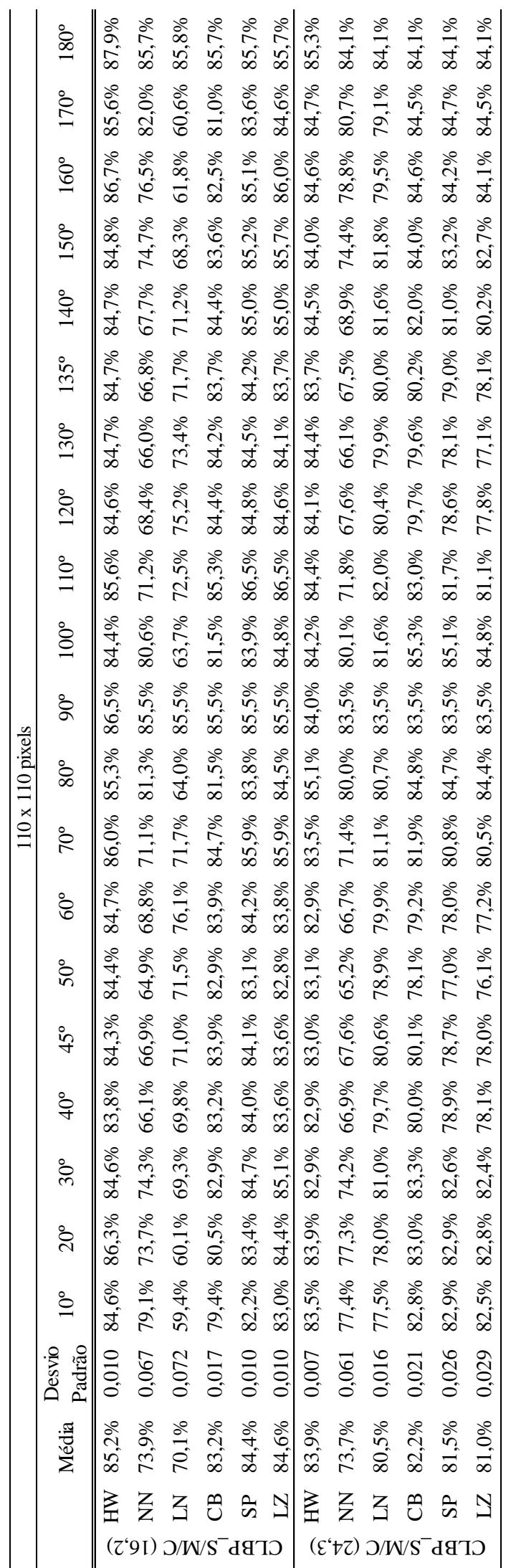




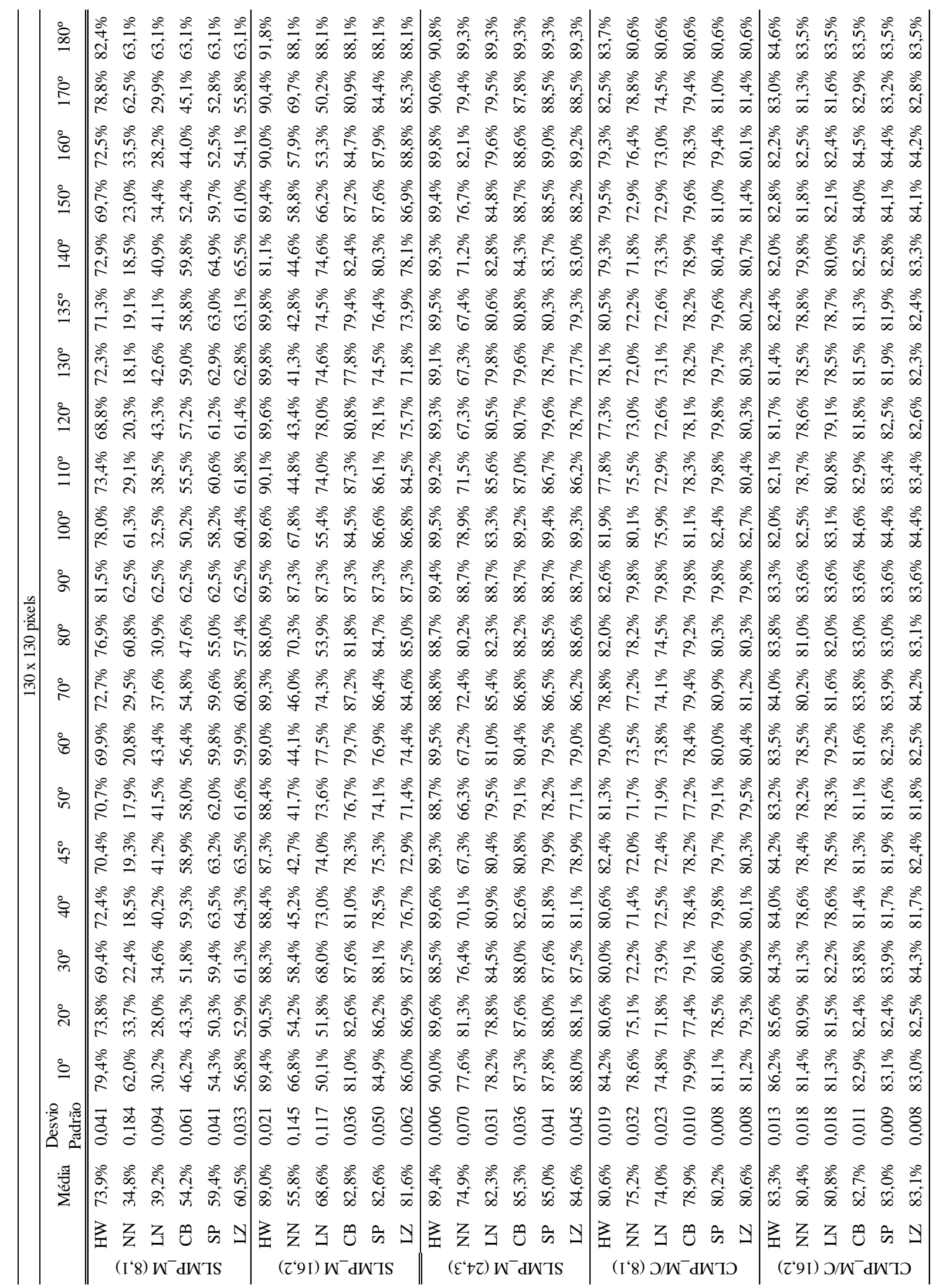




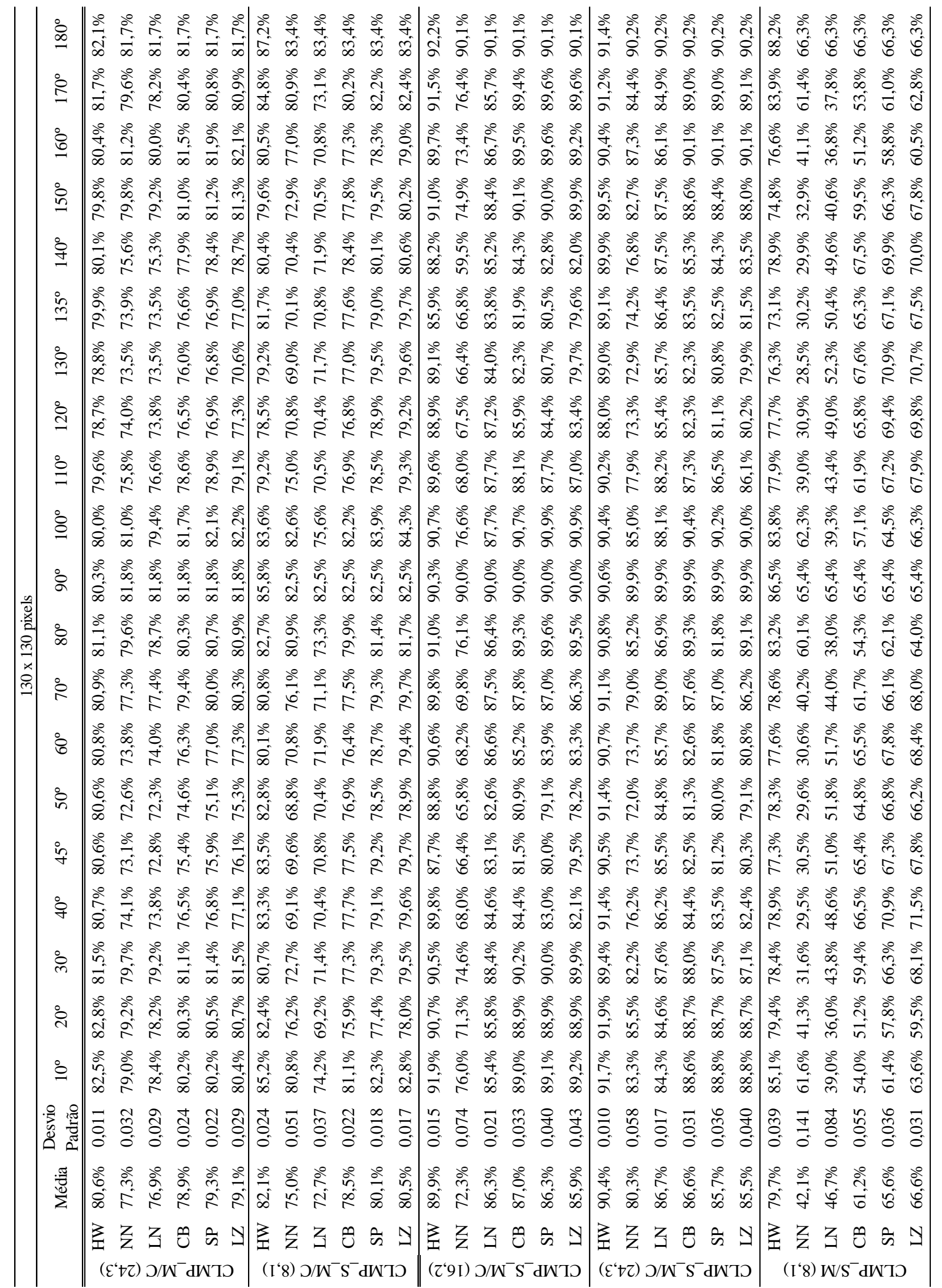




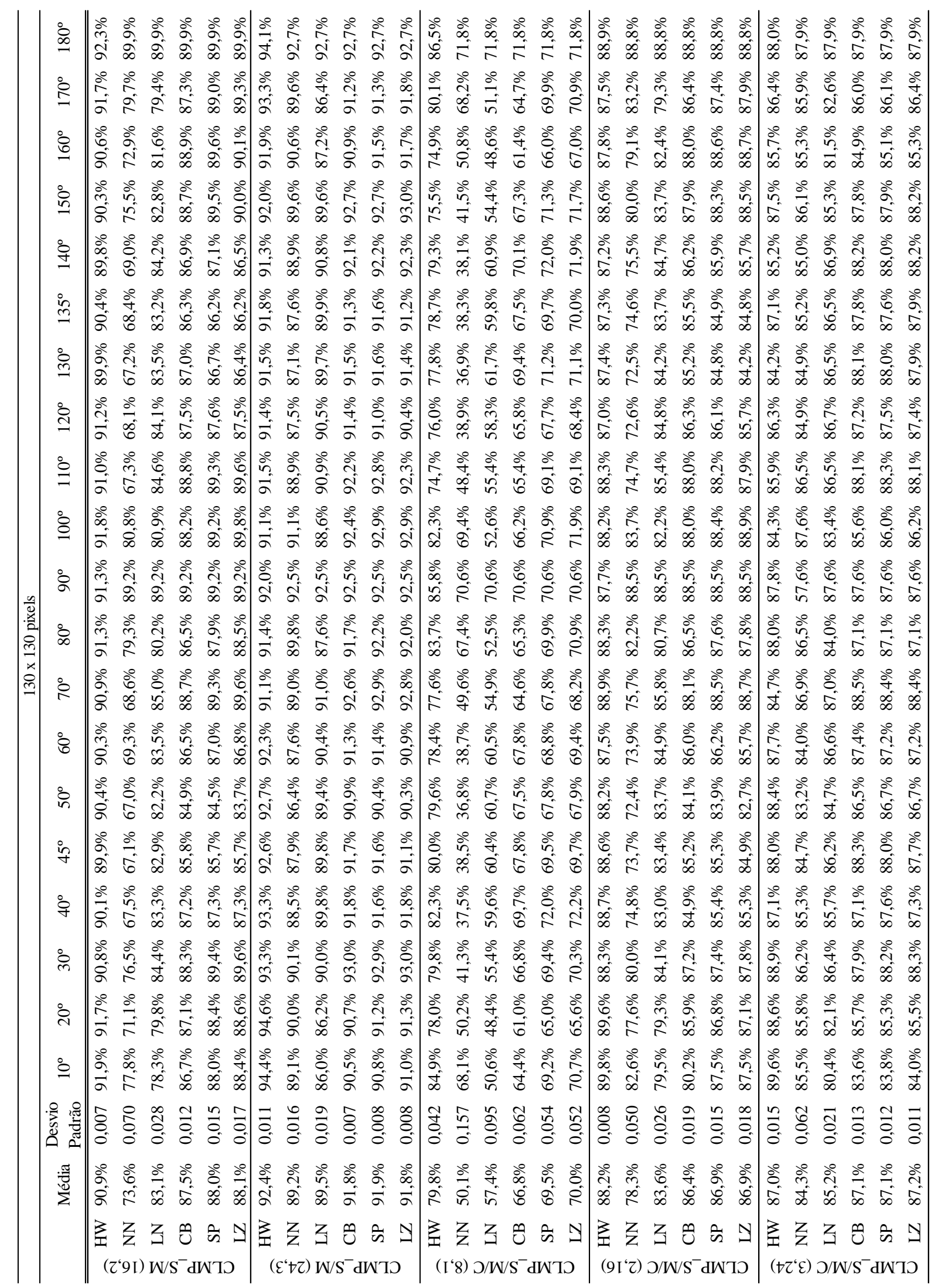




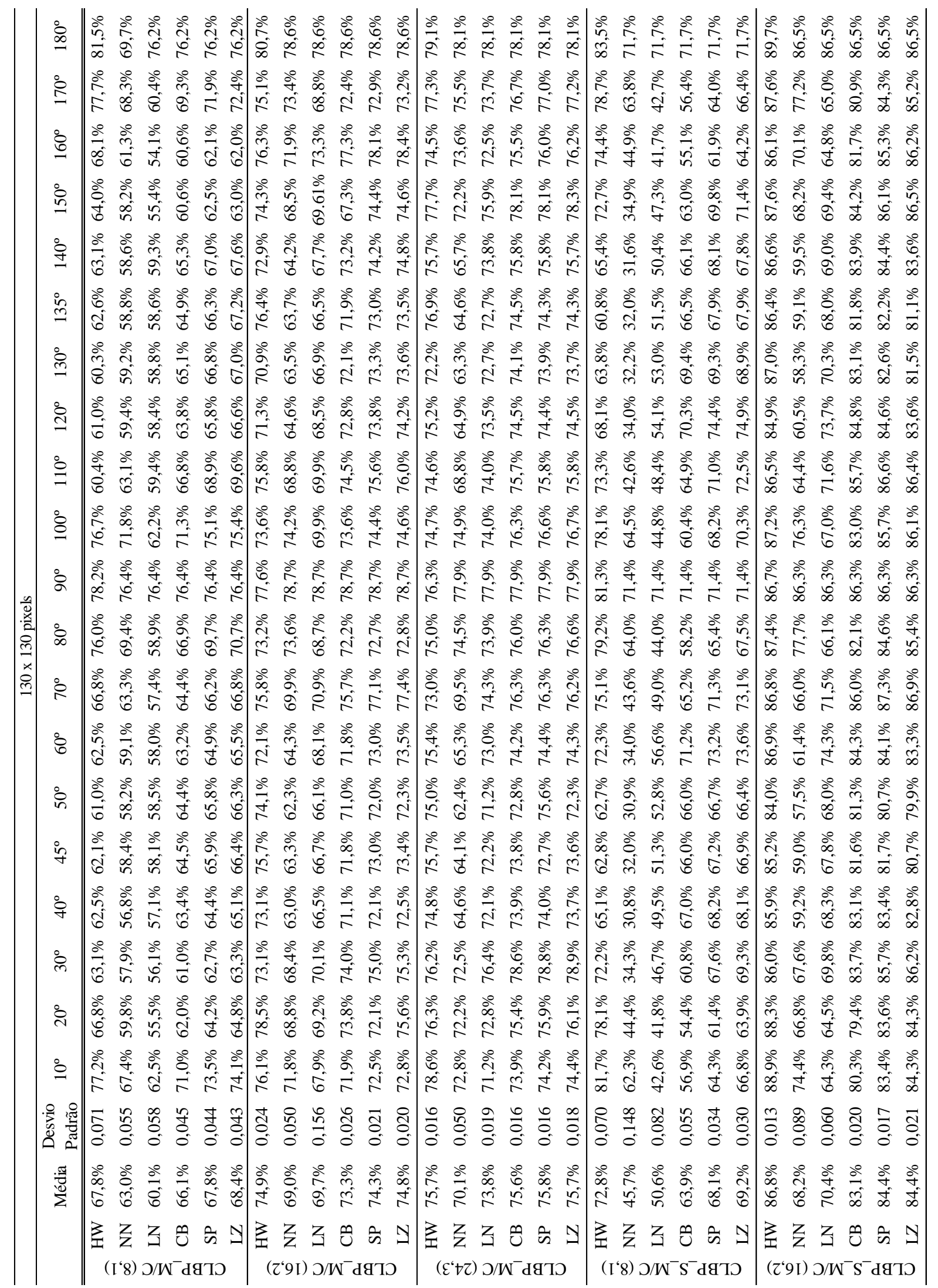




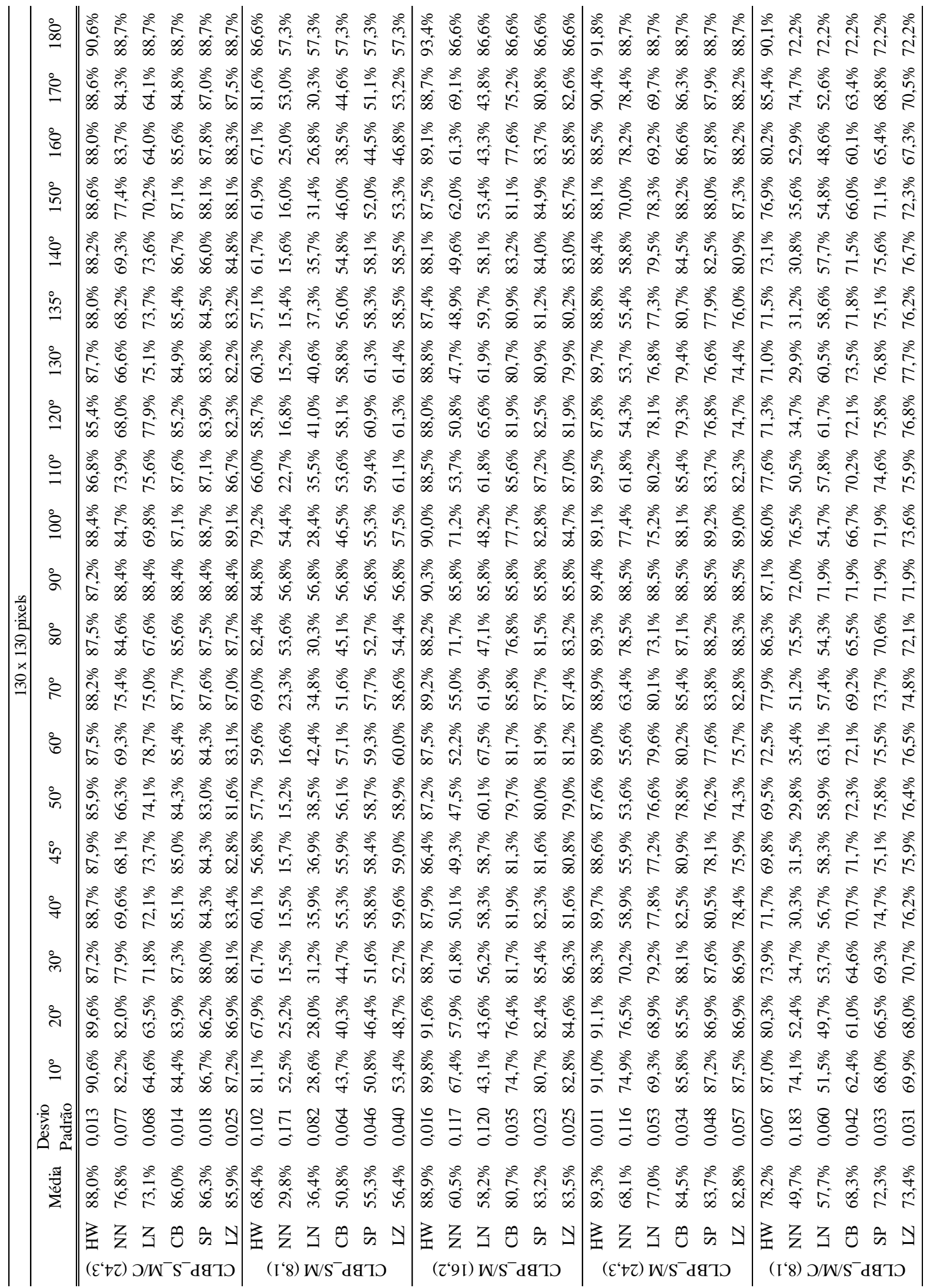




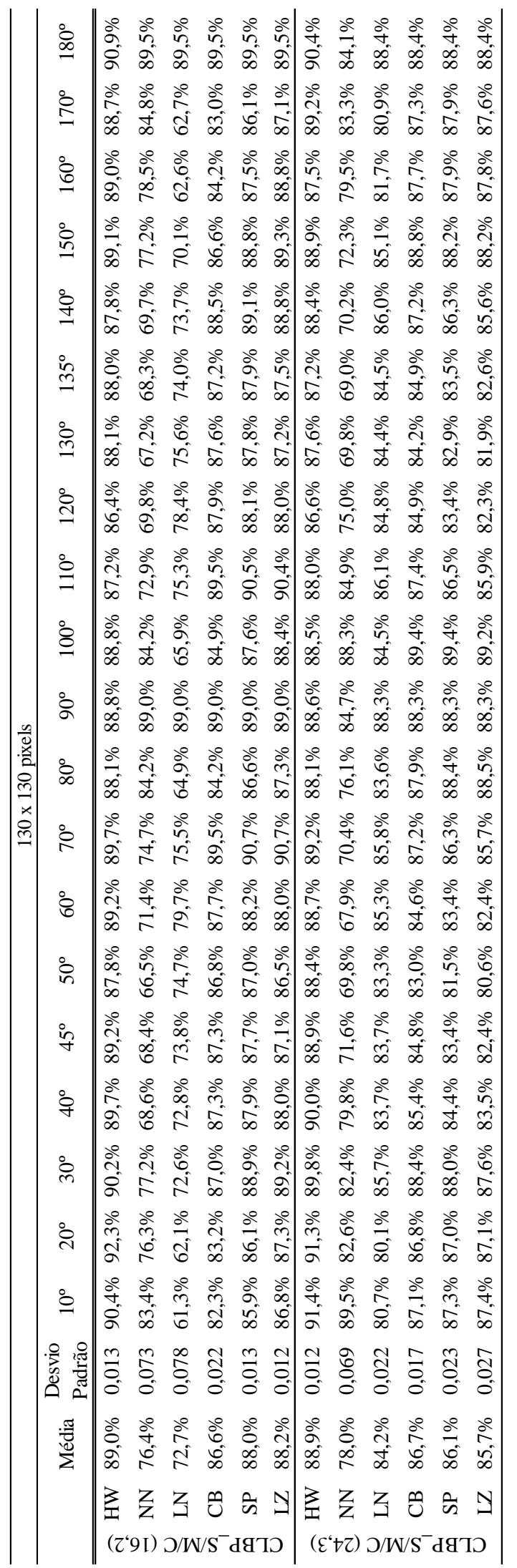

